\title{
2020 Joint conference of the Italian Association of Miology (AIM) and the Italian Association for the study of the Peripheral Nervous System (ASNP), Abstracts
}

Published online: 17 November 2020

(C) Fondazione Società Italiana di Neurologia 2020

Long-Term Safety and Efficacy of Patisiran: Global Open-label Extension 24-month Data in Patients with Hereditary Transthyretin-mediated Amyloidosis

D. Adams ${ }^{1}$, A.González-Duarte ${ }^{2}$, E.Mauricio ${ }^{3}$, T.Brannagan ${ }^{4}$, T.Coelho ${ }^{5}$, J.Wixner ${ }^{6}$, H.Schmidt ${ }^{7}$, A. Mazzeo ${ }^{8}$, E.Berber ${ }^{9}$, M.T Sweetser $^{9}$, M.T White ${ }^{9}$, J.J Wang ${ }^{9}$, M.Polydefkis ${ }^{10}$

${ }^{1}$ National Reference Center for FAP (NNERF)/APHP/INSERM U 1195/ CHU Bicêtre, Le Kremlin Bicêtre, France;

${ }^{2}$ Instituto Nacional de Ciencias Médicas y Nutrición, Salvador Zubirán, Mexico City, Mexico; ${ }^{3}$ Mayo Clinic, Jacksonville, FL, USA;

${ }^{4}$ Department of Neurology, Columbia University, New York City, NY, USA;

${ }^{5}$ Centro Hospitalar Universitário do Porto, Portugal;

${ }^{6}$ Department of Public Health and Clinical Medicine, Umea University, Umeå, Sweden;

${ }^{7}$ University Hospital Muenster, Muenster, Germany;

${ }^{8}$ Unit of Neurology and Neuromuscular Diseases, Department of Clinical and Experimental Medicine, University of Messina, Messina, Italy (presenting on behalf of the authors);

${ }^{9}$ Alnylam Pharmaceuticals, Cambridge, USA;

${ }^{10}$ Johns Hopkins University, Baltimore, USA

Introduction: Hereditary transthyretin-mediated (hATTR) amyloidosis is a progressive, life-threatening disease; most patients develop a mixed phenotype including polyneuropathy and cardiomyopathy. Patisiran's safety/efficacy have been demonstrated in Phase 2 and 3 studies in patients with hATTR amyloidosis with polyneuropathy. Interim 24-month efficacy/safety analyses of the ongoing Global open-label extension (OLE) study are described.

Methods: Multicentre, international, safety and efficacy study (NCT02510261) in eligible patients who completed parent studies, including patients in the Phase 3 APOLLO randomized to placebo (APOLLO/placebo, $n=49)$ or patisiran $(A P O L L O /$ patisiran, $n=137)$ over 18 months and patients in the Phase 2 OLE $(n=25)$ receiving patisiran over 24 months.

Results: $178 / 211$ patients had $\geq 24$ months of exposure as of $07 / 10$ / 2019. Safety profile remained consistent with previous studies. After 24 months of additional patisiran treatment in the Global OLE, durable improvement was seen for modified Neuropathy Impairment Score+7 (mNIS+7) (mean change [SEM]) in APOLLO/patisiran (-4.9 [2.1]) and Phase 2 OLE (-5.9 [2.1]) groups compared to parent study baselines. Norfolk quality of life-diabetic neuropathy (QOL-DN) continued to show durable improvement in APOLLO/patisiran patients (-2.4 [2.4]) following additional 24- month treatment. APOLLO/placebo patients experienced halting of disease progression and QOL improvement compared to Global OLE baseline after 24 months of patisiran in the OLE (mNIS+7: +0.1 [3.3], Norfolk QOL-DN: -4.1 [3.3]), although they had progressed relative to APOLLO baseline (mNIS+7: +26.3 [5.0], Norfolk QOL-DN: +15.8 [4.5]) given the progression while on placebo.

Conclusion: Patients with long-term exposure to patisiran continue to demonstrate durability of efficacy with a positive benefit: risk profile.

Topiramate in Oxaliplatin-Induced Peripheral Neurotoxicity: more than neuroprotection

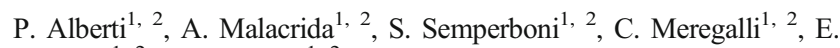
Ballarini $^{1,2}$, G. Cavaletti ${ }^{1,2}$

${ }^{1}$ Experimental Neurology Unit, School of Medicine and Surgery, University of Milano-Bicocca, Monza, Italy.

${ }^{2}$ NeuroMI (Milan Center for Neuroscience), Milan, Italy

Introduction: We demonstrated Topiramate (TPM) prevents Oxaliplatin (OHP) Induced Peripheral Neurotoxicity (OIPN; Alberti et al. 2020) in rats. Moreover, being a carbon anhydrase inhibitor, it can reduce neoangiogenesis and prevent chemoresistance. To verify this, we are searching promising findings in vivo and in vitro.

Methods: In vivo we defined the optimal OHP schedule to induce acute and chronic OIPN in mice, via Nerve Excitability Testing (NET), standard neurophysiology, behavioral test and neuropathology. Via methylthiazolyldiphenyl-tetrazolium bromide (MTT) assay we evaluated the interaction between OHP and TPM in colorectal cancer human cell lines (HCT-15, CaCo2, HT-29).

Results: Male balb/c mice (vehicle treated VS OHP $[7 \mathrm{mg} / \mathrm{Kg}$ i.v., 1qw8ws] treated; $n=12$ in each group) were tested. After the $1^{\text {st }}$ injection acute OIPN was demonstrated via NET in OHP animals. At the end of the 2 months of treatment all outcome measures demonstrated the onset of a relevant chronic sensory neuropathy in OHP group; moreover, on DRG pool collected at the end of treatment, we demonstrated NCX2 downregulation: this shows that acute OIPN was able to induce a persistent despite transient depolarization in neurons. In vitro we didn't find any negative interaction between OHP and TPM; we tested the co-treatment with different OHP dosages (IC25, IC50, IC75 for each cell lines) and TPM $(50,75,100$ microM) in the 3 different colorectal cancer human cell lines. Notably, a promising initial evidence of a cytotoxic effect of TPM was observed in HCT-15 cell line. 
Conclusions: We devise the optimal OHP schedule to reproduce the "bed side" phenomena. We are next moving to test TPM efficacy, against chronic OIPN as well as cancer, in a chronic experiment in tumor bearing immunodeficient mice. Our project has a high potential impact since TPM is a yet approved drug.

A possible biomarker for paclitaxel-induced peripheral neurotoxicity: Neurofilament light chain serum levels monitoring

P. Alberti ${ }^{1}$, F. Cicchiello F ${ }^{2}$, F. Riva ${ }^{2}$, G. Cavaletti ${ }^{1}$, M.E. Cazzaniga ${ }^{2}$

${ }^{1}$ Experimental Neurology Unit, School of Medicine and Surgery, UNIMIB, Monza, Italy

${ }^{2}$ Medical Oncology Unit, San Gerardo Hospital, Monza, Italy

Introduction: Taxane-induced Peripheral Neurotoxicity (TIPN) is a relevant late toxicity that, so far, lacks an efficacious treatment. Neurofilament light chain (NfL) serum levels have emerged as a potential biomarker in neurological diseases and have shown promising usefulness in detecting chemotherapy induced peripheral neurotoxicity in animal models (Meregalli et al.2018). We aimed to explore, in a pilot study, NfL dosing in TIPN patients.

Methods: We enrolled breast cancer patients: 10 patients (group A) before treatment and 9 patients (group B) after. TNSc@ and neurophysiology were used to assess neurological status. Serum NfL levels were determined as described by Rohrer et al., 2016.

Results: All patients had no other conditions predisposing to peripheral neuropathy. Neurological examination was normal for all subjects in group A. In group B TNSc $\odot$ mean score was 4; the majority of group $B$ patients showed a grade I TIPN (i.e, TNSc@ score lower than 8 ) and 1 had a grade II TIPN (i.e., TNSc@ $\odot$ higher than 8). Median NfL serum levels were $21.05 \mathrm{pg} / \mathrm{mL}$ in group A and $112.3 \mathrm{pg} / \mathrm{mL}$ in Group B. Mann Withney U-test showed a significant difference between the 2 groups $(\mathrm{p}<0.001)$ for both variables.

Conclusions: In our small population we compared no neuropathy and mild TIPN patients. Despite the mild neurological burden, NfL levels were significantly increased in TIPN patients. Thus, NfL levels could increase as an early damage ensues. Therefore, we are planning a larger longitudinal study to identify a cut-off to predict - early (after 2 nd cycle) TIPN onset and course.

Pharmacological chaperone to treat myotonia congenita caused by trafficking-defective CIC-1 chloride channel mutants: proof-ofconcept with niflumic acid

C. Altamura ${ }^{1}$, E. Conte ${ }^{2}$, D. Sahbani ${ }^{2}$, G.M Camerino ${ }^{3}$, F. Girolamo ${ }^{2}$, MR. Carratù ${ }^{1}$, P. Imbrici ${ }^{2}$, JF. Desaphy ${ }^{1}$

${ }^{1}$ Dept. of Biomedical Sciences and Human Oncology, University of Bari Aldo Moro

${ }^{2}$ Dept. of Pharmacy - Drug Sciences, University of Bari Aldo Moro

3 Dept. of Basic Medical Sciences, Neurosciences and Sense Organs, University of Bari Aldo Moro

Myotonia congenita (MC) is a skeletal muscle channelopathy caused by loss-of-function mutations in $\mathrm{ClC}-1$ chloride channel, which impaired channel function or membrane expression. One promising strategy may be to restore $\mathrm{ClC}-1$ surface expression by using pharmacological chaperones able to stabilize misfolded mutated proteins. Thus, we tested the ability of two reversible $\mathrm{ClC}-1$ ligands, 9-anthracene-carboxylic acid(9-AC) and niflumic acid(NFA), to restore membrane expression of trafficking-defective MC mutants(p.G411C,p.A531V,p.V947E).
Wild-type(WT) or MC mutant ClC-1 channels were expressed in HEK293 cells and whole-cell chloride currents were recorded with patch-clamp. Membrane biotinylation assays and confocal imaging were performed to support electrophysiological results.

Expression of A531V and V947E channel mutants yield a drastic reduction of chloride current amplitude, while no current was detected in G411C-transfected cells. Biotinylation assay and confocal imaging revealed that the vast majority of G411C mutant channels were retained inside the cells. Incubation of transfected cells for 24 hours with 9-AC(30 $\mu \mathrm{M})$ or NFA $(50 \mu \mathrm{M})$ enhanced A531V and V947E chloride current density more than two-fold, thereby restoring currents similar to WT. Conversely, NFA incubation did not allow to record chloride currents in G411C-transfected cells, suggesting NFA inability to exert chaperone effect or $\mathrm{G} 411 \mathrm{C}$ inability to conduct chloride currents. Incubation of G411C-transfected cells with the proteasome inhibitor MG132 $(20 \mu \mathrm{M})$ for 16hours rescued the surface expression level of G411C mutant. However, no significant chloride current was recorded after MG132 incubation, suggesting that this mutation produced nonfunctional $\mathrm{ClC}-1$ channels.

The results with 9-AC and NFA could be the starting point for development of a new personalized treatment for MC patients.

\section{A diagnostic delay of case of neurogastrointestinal encephalopathy} (MNGIE)

F. Aruta ${ }^{1}$; G. Capaldo ${ }^{2}$;C. Pelosi ${ }^{3}$; A. Iovino ${ }^{1}$; M. Giaquinto ${ }^{1}$; P. Romano ${ }^{4}$; R. Rinaldi ${ }^{5}$; S. Trimarco ${ }^{1}$; F. Vitale ${ }^{1}$; L. Ruggiero ${ }^{1}$

${ }^{I}$ Dipartimento di Neuroscienze, Scienze Riproduttive ed Odontostomatologiche, Università Federico II di Napoli

${ }^{2}$ Reparto Neurologia Presidio Ospedaliero Ariano Irpino, Avellino

${ }^{3}$ Reparto Medicina Interna Presidio Ospedaliero Ariano Irpino, Avellino

${ }^{4}$ Reparto Radiologia Presidio Ospedaliero Sant'Angelo dei Goti, Benevento

${ }^{5}$ Reparto di Neurologia, Sant'Orsola-Malpighi Ospedale Universitario, Bologna

The gastrointestinal symptoms are possible complication in neurological diseases and sometimes the first manifestation of some rare genetic syndrome as mitochondrial neurogastrointestinal encephalopathy (MNGIE). However this association are frequently unrecognized especially by physician without a specific expertise in rare diseases.

A Thirty-two years old woman was evaluated from the juvenile age in a gastroenterology division for a severe weight loss due to an unexplained malabsorption syndrome but the laboratory and endoscopic screening had not been not contributive. Moreover, due to a several vomiting episodes she developed recurrent aspiration pneumonia and had needed in an intensive care division. However her clinical picture was characterized by several neurological signs that had been neglected. The patient showed bilateral ptosis with ophthalmoplegia, dysphagia for liquids and widespread weakness and waddling gait. So she was underwent to an electrophysiological study and we identified an axonal sensory polyneuropathy with normal electromyography. Brain MRI showed severe bilateral confluent leukoencephalopathy with cortical and subcortical white matter alterations. Finally muscle biopsy showed several fibers with oxidative metabolism deficiency. Considering the combination of neurological features, mitochondrial alterations on muscle biopsy and gastrointestinal impairment, we suspected the mitochondrial neurogastrointestinal encephalopathy (MNGIE) syndrome. So the genetic analysis was performed and was identified the homozygous c.1160-1G>A mutation on the TYMP gene. The neurological genetic diseases frequently are multisystem pathology, it would be desirable to have a greater awareness of this possibility also for physician without an expertise in rare genetic disease. 
Automatic recognition of ragged red fibers in muscle's histological images of patients affected by mithocondrial disorders

\author{
J. Baldacci ${ }^{1}$, M. Calderisi ${ }^{1}$, A. Rubegni ${ }^{2}$, C. Fiorillo ${ }^{3}$, F.M. Santorelli ${ }^{2}$ \\ ${ }^{1}$ Kode Solutions, Pisa, Italy \\ ${ }^{2}$ IRCCS Fondazione Stella Maris, Pisa, Italy \\ ${ }^{3}$ IRCCS G. Gaslini, Genova, Italy
} that occur when mitochondria fail to produce enough energy for the body to function properly. One of the most important histological pattern of mitochondrial disease are the ragged red fibers. Their characteristic appearance is due to the accumulation of abnormal mitochondria below the membrane of the muscle fiber.

The aim of our study was to develop and validate machine-learning methods for ragged red fibers detection in light microscope images of the skeletal muscle tissue. We used image sets of 489 color images captured from representative areas of Gomori's trichrome stained tissue retrieved from light microscope images at 20x magnification.

All the sub-images were used as input for generating features. The generated features were grouped into color features, texture features and wavelet transform features.

We performed a double-step binary classification. The first step aimed to distinguish the muscular tissue from the not relevant zones of the images. The second step aimed to distinguish the ragged red fibers from the "healthy" muscular fibers.

We compared the performance of random forest, gradient boosting machine and support vector machine classifiers. On 'tissue-waste' classification, using a ten-fold cross-validation estimate, an accuracy of $91.1 \%$ was obtained. On the second step, the achieved accuracy of classification into ragged and not-ragged classes was $96.3 \%$. Our data suggest that the advent of the scanning technologies, combined with the development of machine learning models for image classification, make neuromuscular disorders' automated diagnostic systems a concrete possibility

Clinical and genetic study in a patient with Neutral Lipid Storage Disease with Myopathy (NLSD-M)

E. Baldelli ${ }^{1}$, S. Cotti Piccinelli ${ }^{1}$, B. Risi ${ }^{1}$, N. Necchini ${ }^{1}$, A. Galvagni ${ }^{1}$, A. Padovani ${ }^{1}$, M. Filosto ${ }^{1}$

${ }^{1}$ Center for Neuromuscular Diseases, Unit of Neurology, ASST Spedali Civili and University of Brescia, Brescia, Italy

Neutral lipid storage disease with myopathy (NLSD-M) is an autosomal recessive muscle disorder characterized by triglyceride-containing cytoplasmic droplets in leukocytes and muscle tissue. It presents with adult onset slowly progressive proximal muscle weakness often associated with hypertrophic cardiomyopathy.

Here, we report a 42-year-old patient who presented progressive proximal upper limb weakness since age 38 .

Clinical examination showed asymmetrical (right $>$ left) proximal upper limb weakness and muscle hypotrophy, mild weakness of both orbicularis oculi muscles and difficulty in walking on heels.

Diffuse accumulation of lipid droplets in muscle cells was detected on muscle biopsy and Jordan anomaly, a well-known NLSD marker, was revealed by peripheral blood smear.

Genetic analysis displayed a novel homozygote deletion in the PNPLA2 gene (exon2: c [45 47de]) causing the in frame p.[Gly16Del]. Both unaffected parents harbored the heterozygote deletion.

Upper-limb muscle MRI showed atrophy of the supraspinatus and infraspinatus muscles, especially on the right side. Cardiac focal inferolateral intramural fibrosis was noted by MRI as a possible manifestation of lipid accumulation. However, no cardiac involvement was clinically detected.

Our study confirms that 1) distal muscle involvement can be a feature of NLSD-M, 2) in Italian patients cardiac involvement is usually mild, differently from Far East subjects in which it is frequent and often leads to heart transplantation, 3) infraspinatus muscle is one of the most radiologically affected muscles and 4) Italian families usually harbor private mutations. Moreover, our patient presents with orbicularis oculi muscle weakness, therefore indicating that facial muscles can also be involved and confirming clinical heterogeneity of this disease.

Mapping the neurotoxic effect of Oxaliplatin on dorsal root ganglia: MALDI mass spectrometry imaging highlights alterations to the tissue proteome

E. Ballarini ${ }^{1}$, P. Alberti ${ }^{1}$, V. Carozzi ${ }^{1}$, V. Rodriguez-Menendez ${ }^{1}$, A. Smith ${ }^{2}$, G. Cavaletti ${ }^{1}$, F. Magni ${ }^{2}$

${ }^{1}$ Department of Medicine and Surgery, Experimental Neurology Unit, University of Milano-Bicocca, Monza, Italy.

${ }^{2}$ Department of Medicine and Surgery, Clinical Proteomics and Metabolomics Unit, University of Milano-Bicocca, Monza, Italy.

Chemotherapy-induced peripheral neurotoxicity is a common side effect in cancer patients. Different antineoplastic drugs have distinctive toxicity profiles: Oxaliplatin determines its primary neurotoxic effect on dorsal root ganglia (DRGs) sensory neurons while Bortezomib primarily affects peripheral nerves and, to a small extent, also DRGs.

In order to evaluate the possible modification of the tissue proteome after antineoplastic drugs treatment, MALDI Mass Spectrometry Imaging (MALDI-MSI) analysis was performed on $3 \mu \mathrm{m}$ thick tissue sections of formalin fixed paraffin-embedded DRGs dissected from male Balb/c mice chronically treated with Oxaliplatin, Bortezomib and controls.

The onset of a relevant peripheral neurotoxic effect in Balb/c mice was determined through neurophysiology, behavioural tests and neuropathology. For MALDI-MSI analyses, all mass spectra were obtained in the $\mathrm{m} / \mathrm{z}$ 700 to 3000 range using a rapifleX MALDI Tissuetyper ${ }^{\mathrm{TM}}$ and the images were acquired using a lateral resolution of $10 \mu \mathrm{m}$.

Initially, principal component analysis evidenced alterations in the DRG tissue proteome of those mice treated with Oxaliplatin while no alterations were observed in the DRGs of mice treated with Bortezomib whose proteome was considered similar to that of the controls. Additionally, a number of individual protein signals (AUC $\geq 0.8$ ) had an altered intensity in the DRGs of mice treated with Oxaliplatin and these signals were localized both to DRG sensory neurons and dorsal roots.

MALDI-MSI analyses highlighted Oxaliplatin-induced tissue proteome alterations reflecting its primary neurotoxic effect on DRGs. The absence of significant Bortezomib-induced proteome modifications reflected its primary neurotoxic effect on axons, further supporting the reliability of this method.

FIREFISH Part 2: Efficacy and safety of risdiplam (RG7916) in infants with Type 1 spinal muscular atrophy (SMA)

G. Baranello, ${ }^{1,2}$ L. Servais,${ }^{3,4}$ R. Masson, ${ }^{1}$ M. MazurkiewiczBełdzińska, ${ }^{5}$ K. Rose, ${ }^{6}$ D. Vlodavets, ${ }^{7}$ H. Xiong, ${ }^{8}$ E. Zanoteli, ${ }^{9}$ M. ElKhairi, ${ }^{10}$ S. Fuerst-Recktenwald, ${ }^{11}$ M. Gerber ${ }^{12}$ K. Gorni, ${ }^{13}$ H. Kletzl,${ }^{14}$ R. Scalco, ${ }^{11}$ B. T. Darras ${ }^{15}$ on behalf of the FIREFISH Working Group

${ }^{I}$ Fondazione IRCCS Istituto Neurologico Besta, Developmental Neurology Unit, Milan, Italy;

${ }^{2}$ The Dubowitz, Neuromuscular Centre, UCL Great Ormond Street Institute of Child Health, London, UK (current); 
${ }^{3}$ Reference Center for Neuromuscular Disease, Centre Hospitalier Régional de La Citadelle, Liège, Belgium;

${ }^{4}$ MDUK Neuromuscular Centre, Department of Paediatrics, University of Oxford, UK;

${ }^{5}$ Department of Developmental Neurology, Medical University of Gdańsk, Gdańsk, Poland;

${ }^{6}$ Paediatric Gait Analysis Service of New South Wales, The Children's Hospital at Westmead Sydney, Australia;

${ }^{7}$ Russian Children Neuromuscular Center, Veltischev Clinical Pediatric Research Institute of Pirogov Russian National Research Medical University, Moscow, Russia;

${ }^{8}$ Department of Pediatrics, Peking University First Hospital, Beijing, China;

9 Department of Neurology, Hospital das Clínicas, University of São

Paulo, São Paulo, Brazil;

${ }^{10}$ Roche Products Ltd., Welwyn Garden City, UK;

${ }^{11}$ Pharma Development Neurology, F. Hoffmann-La Roche Ltd., Basel, Switzerland;

12 Pharma Development, Safety, F. Hoffmann-La Roche Ltd, Basel, Switzerland;

${ }^{13}$ PDMA Neuroscience and Rare Disease, F. Hoffmann-La Roche Ltd., Basel, Switzerland;

${ }^{14}$ Roche Pharmaceutical Research and Early Development, Roche Innovation Center Basel, Basel, Switzerland;

15 Boston Children's Hospital, Harvard Medical School, Boston, MA, USA.

SMA is a severe, progressive neuromuscular disease caused by reduced levels of survival of motor neuron (SMN) protein due to deletions and/or mutations of the $S M N 1$ gene. A second gene, $S M N 2$, produces only low levels of functional SMN protein. Risdiplam (RG7916) is a centrally and peripherally distributed oral $S M N 2$ pre-mRNA splicing modifier that increases levels of functional SMN protein.

FIREFISH (NCT02913482) is an ongoing, multicenter, open-label study of risdiplam in infants aged 1-7 months at enrollment with Type 1 SMA and two SMN2 gene copies. FIREFISH Part $1(\mathrm{n}=21)$ assesses the safety, tolerability and PK/PD of different risdiplam dose levels. In FIREFISH Part 1 there have been no drug-related safety findings leading to withdrawal from the study following $\leq 30$ (median 19) months of treatment (data-cut: 2nd July 2019). The primary objective of confirmatory Part 2 $(n=41)$ is to investigate the efficacy of risdiplam at the dose selected in Part 1 . The primary efficacy endpoint is the proportion of infants sitting without support for 5 seconds after 12 months on treatment, as assessed by Item 22 of the Gross Motor Scale of the BSID-III. Additional secondary endpoints will also be measured.

Here we will report efficacy and safety data from the confirmatory Part 2 of the FIREFISH study in participants who have received treatment with risdiplam for a minimum of 12 months at the dose selected in Part 1.

Part 2 of FIREFISH will provide important data on the efficacy and safety of risdiplam in infants with Type 1 SMA.

\section{Long-term functional changes in Becker muscular dystrophy}

L. Bello, S. Mastellaro, L. Caumo, P. Riguzzi, V. Zangaro, M. Villa, D. Sabbatini , A. Fusto, B. Merlo, S. Vianello, E. Pegoraro

Neuromuscular Unit, Department of Neurosciences DNS, University of Padova

Introduction: Clinical trial design in Becker muscular dystrophy (BMD) requires a quantitative description of functional changes over time, in different age and mutation groups.

Material and methods: We applied linear mixed models (LMM) to longitudinally collected functional data regarding 6 Minute Walk Test (6MWT), North Star Ambulatory Assessment (NSAA), and Timed Function Tests (TFTs): run/walk $10 \mathrm{~m}$ (TRUN), rise from the floor
(TRISE), climb 4 standard steps (TCLIMB), collected in the BMD cohort followed at our Center from 2012 to 2020.

Results and discussion: We collected functional data from $97 \mathrm{BMD}$ patients, 77 aged 18 or older, average age $30.8 \pm 17.4$ years. Average follow-up was $4.2 \pm 2.6$ years (maximum 8 years), with $4.9 \pm 2.4$ evaluations (maximum 9), for a total of 480 evaluations. The most frequent mutations were del 45-48 ( $\mathrm{n}=16)$, del 45-47 $(\mathrm{n}=14)$, del $48(\mathrm{n}=12)$, del 45-51 $(\mathrm{n}=8)$, and del 45-55 $(n=5)$. All outcomes significantly decreased with age in adult patients: NSAA $-0.67 \pm 0.05$ points/year, $\mathrm{p}<0.0001 ; 6 \mathrm{MWT}-2.3 \pm 0.8 \mathrm{~m} /$ year, $\mathrm{p}=$ 0.0062 ; TRUN $-0.036 \pm 0.008 \mathrm{~m} / \mathrm{s} *$ year, $\mathrm{p}<0.0001$; TRISE $-0.007 \pm 0.002$ $\mathrm{s}^{-1} /$ year; and TCLIMB $-0.034 \pm 0.006$ steps $/ \mathrm{s}^{*}$ year, $\mathrm{p}<0.0001$. Functional measures did not decrease with age in several mutation groups, including deletions ending on exon 51 and exon 48.

Conclusion: We confirm genotype/phenotype correlations and provide mutation-specific, quantitative natural history data to be applied to BMD clinical trial design.

\section{A case of a Heterozygous Kv1.1 N255D Mutation with Normal Serum} Magnesium Levels

\author{
F. Bianchi ${ }^{1}$, C. Simoncini ${ }^{1}$, G. Ricci ${ }^{1}$, P. Bernasconi ${ }^{2}$, G. Siciliano ${ }^{1}$ \\ ${ }^{I}$ Department of Clinical and Experimental Medicine, Neurological \\ Clinic, University of Pisa, Pisa, Italy \\ ${ }^{2}$ Neurology IV-Neuroimmunology and Neuromuscular Diseases Unit, \\ Fondazione IRCCS Istituto Neurologico Carlo Besta, Milan, Italy
}

Mutations of the main voltage-gated $\mathrm{K}$ channel members Kv1.1 are linked to several clinical conditions, such as periodic ataxia type 1 and myokimia. Due to its role in active magnesium reabsorption through the renal distal convoluted tubule segment, mutations in this protein have been reported to cause hypomagnesemia with muscular symptoms. In particular, the amino acid substitution of a highly conserved asparagine for an aspartic acid in the third transmembrane segment of Kv1.1 (p.Asn255Asp) results in a nonfunctional channel, leading to an autosomal dominant hypomagnesemia associated to muscle cramps, tetanic episodes, and muscle weakness responsive to magnesium supplementation, as described in a single large family. Here we report the case of a young female patient who came to our attention for a history started in infancy of muscular spasms, tetanic episodes and muscle weakness, initially misdiagnosed for fibromyalgia. After a genetic screening she was found to be carrier of the c.736A $>\mathrm{G}$ (p.Asn255Asp) mutation in the KCNA1 gene encoding for Kv1.1. Contrarily to what previously described in literature, our patient has always presented normal serum and urinary magnesium values. She rather was affected by a slight hypocalcemia, so that calcium supplementation was the only treatment that provided her a clinical benefit, while she referred a worsening of her muscular symptoms with magnesium integration. The genetic test for mutations in exon 1 of $K C N A 1$ on both patient's parents resulted negative suggesting at a first instance that in our patient the mutation in KCNAl appeared de novo.

Clinical, pathological and prognostic heterogeneity in immunecheckpoint inhibitors-induced myositis

S. Bocci ${ }^{1}$, N. Volpi ${ }^{1}$, R. Danielli ${ }^{2}$, S. Bartalini ${ }^{1}$, L. Calabrò ${ }^{2}$, A. M. Di Giacomo $^{2}$, M. Maio $^{2}$, F. Giannini ${ }^{1}$

${ }^{I}$ Department of Medicine, Surgery and Neuroscience, University of Siena, Siena, Italy

${ }^{2}$ Center for Immuno-Oncology, University Hospital of Siena, Siena, Italy

Treatment with immune-checkpoint inhibitors (ICIs), including monoclonal antibodies against programmed death-1 (PD-1) and its ligand (PDL-1), is approved in many tumor types. By unbalancing 
immune system, ICIs may generate several multi-organ immunerelated Adverse Events (irAEs), including neuromuscular manifestations.

Among 406 patients with solid tumors treated with ICIs in Siena's Center for Immuno-Oncology between 2013 and 2019, we identified $4(<1 \%)$ metastatic melanoma patients presenting clinical, electromyographic and laboratory findings suggestive for myopathy, alone or associated to other neurological irAEs. All patients underwent muscular biopsy.

Patient 1 presented ptosis, fluid dysphagia, myalgias and lower limbs weakness after first anti-PD1 administration. Muscular biopsy showed granulomatous myositis. Specific antibodies and repetitive nerve stimulation showed concomitant Myasthenia Gravis (MG). Patient recovered in 7 weeks with oral steroids. Patient 2 presented dropped head, bilateral ptosis, hypophonia, fatigue and dyspnea after second anti-PD1 administration. Muscular biopsy showed necrotizing myositis with minimum inflammation. Specific antibodies showed concomitant MG. Patient required non-invasive ventilation and intravenous and oral steroids, slowly recovering within 6 months. Patients 3 and 4 showed polymyositis-like pathological pattern after anti-PD1 therapy, with markedly different courses: the first had mild disease, fully recovered in 3 months with oral steroid, whereas the second had severe and prolonged course, requiring hospitalization, invasive ventilation and multiple immunoactive therapies.

ICIs-induced myositis can present with different clinical and pathological features, isolated or associated to MG. Ocular muscles are frequently involved (4/4 in our series) regardless MG co-morbidity. Severity, course and prognosis are heterogeneous and apparently unrelated to different pathological patterns.

Peripheral Nervous System (PNS) toxicity induced by immunecheckpoint inhibitors in cancer patients: single centre experience

S. Bocci ${ }^{1}$, L. Insana ${ }^{1}$, R. Danielli ${ }^{2}$, F. Ginanneschi ${ }^{1}$, L. Franci ${ }^{1}$, L. Calabrò ${ }^{2}$, A. M. Di Giacomo ${ }^{2}$, M. Maio ${ }^{2}$, F. Giannini ${ }^{1}$

${ }^{1}$ Department of Medicine, Surgery and Neuroscience, University of Siena, Siena, Italy

${ }^{2}$ Center for Immuno-Oncology, University Hospital of Siena, Siena, Italy

Treatment with immune-checkpoint inhibitors (ICIs), including monoclonal antibodies against CTLA-4, PD-1 and PDL-1, is approved in many tumor types. By unbalancing immune system, ICIs may generate several multi-organ immune-related Adverse Events (irAEs), including neuromuscular manifestations.

We describe five patients suffering from metastatic melanoma and one from non-small-cell lung cancer who experienced PNS-irAEs after ICIs therapy. Three patients presented sensory-motor axonal polyneuropathies, two of which with mild and slow course, onset after 13 and 16 weeks of therapy and followed by spontaneous recovery in 4 and 12 months respectively. Third patient presented subacute course with associated myopathy, beginning after one week of treatment and improved after discontinuation and steroid therapy. Fourth patient presented rapidly ascending tetraparesis at week 6 of immunotherapy. Lab tests and EDX were diagnostic for AIDP. Full recovery was obtained after IVIg treatment. Fifth patient presented focal onset in left lower limb followed by severe spreading of signs and loss of deambulation after two sequential immunotherapies. EDX showed CIDP-like abnormalities. ICIs withdrawal and IVIg plus steroids administration caused moderate benefit. Last patient presented oftalmoparesis and diffuse weakness after 3 weeks of treatment. EDX were consistent with CIDP plus ocular nerve involvement. Immunotherapy discontinuation and steroids administration were ineffective. Anti-gangliosides and anti-onconeural antibodies were negative in all patients.
The current series quite represents the whole clinical spectrum of PNSirAEs, ranging from mild and spontaneously or treatment reversed to poorly responsive complications. Increasing use of ICIs needs joined specific alert by oncologists and neurologists, to promptly recognize and treat PNS-irAEs.

Myoglobinuria as unexpected onset of disease in an asymptomatic 75-years-old man

\author{
C. Bonanno ${ }^{1}$, A. Pugliese ${ }^{1}$, G. Nicocia ${ }^{1}$, C. Rodolico ${ }^{1}$, A. Toscano ${ }^{1}$ \\ ${ }^{1}$ Department of Clinical and Experimental Medicine, Unit of Neurology \\ and Neuromuscular Diseases, University of Messina, Messina, Italy
}

A 75 years-old man self-referred to the Emergency Department for generalized fatigue associated with heart palpitations and cough. Thorax x-ray resulted normal whilst the electrocardiogram showed atrial fibrillation and a treatment with intravenous amiodarone was promptly started with conversion to sinus rhythm. Routine laboratory analysis were in normal range except for a slight increase in troponin T and hyperCKemia elevated up to $10000 \mathrm{UI} / 1$ (0 200 UI/l). Because of hyperCKemia patient was referred to Our Department. Medical history revealed a 15-days history of dark urine ("dark orange"). No comorbidities or drugs taken. Further laboratory tests revealed elevated ERS rate and myoglobinuria. At neurological examination neck flexors and proximal lower limb weakness was detected. Electromyography (EMG) showed a myopathic pattern on both upper and lower limbs muscles without spontaneous activity. Muscle biopsy guided us to the diagnosis which has been confirmed after one month follow up.

\section{Immune-mediated necrotizing myopathies: clinical-serological fea-} tures of a large Italian cohort of patients

S. Bonanno ${ }^{1}$, M. Lucchini ${ }^{2}$, E. Pancheri ${ }^{3}$, P. Rovere Querini ${ }^{4}$, R. De Lorenzo $^{4}$, A. Biglia ${ }^{5}$, C. Gemelli ${ }^{6}$, P. Riguzzi ${ }^{7}$, L. Bello ${ }^{7}$, A. Pugliese ${ }^{8}$, L. Ruggiero ${ }^{9}$, G. Ricci ${ }^{10}$, C. Fiorillo ${ }^{11}$, G. Vattemi ${ }^{3}$, G. Siciliano ${ }^{10}$, C. Rodolico $^{8}$, M. Filosto ${ }^{12}$, M. Grandis ${ }^{6}$, M. Garibaldi ${ }^{13}$, E. Pegoraro ${ }^{7}$, S.

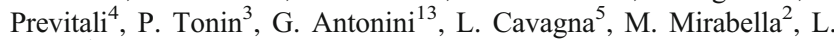
Maggi ${ }^{1}$.

${ }^{1}$ Fondazione IRCCS Istituto Neurologico Carlo Besta, Milano, Italy

${ }^{2}$ Fondazione Policlinico Universitario Agostino Gemelli IRCCS, Roma, Italy

${ }^{3}$ Università degli Studi di Verona, Verona, Italy

${ }^{4}$ IRCCS Ospedale San Raffaele, Milano, Italy

${ }^{5}$ Fondazione IRCCS Policlinico San Matteo, Pavia, Italy

${ }^{6}$ Università degli Studi di Genova, Genova, Italy

${ }^{7}$ Università degli Studi di Padova, Padova, Italy

${ }^{8}$ Università degli Studi di Messina, Messina, Italy

${ }^{9}$ Università di Napoli Federico II, Napoli, Italy

${ }^{10}$ Università di Pisa, Pisa, Italy

${ }^{11}$ Istituto Giannina Gaslini, Genova, Italy

${ }^{12}$ ASST Spedali Civili di Brescia, Brescia, Italy

${ }^{13}$ Sapienza Università di Roma, Ospedale Sant'Andrea, Roma, Italy

Immune-mediated necrotizing myopathies (IMNMs) represent a heterogeneous group of muscle disorders recently identified within the spectrum of idiopathic inflammatory myopathies (IIMs) by distinctive clinical, pathological, serological, and therapeutic features. Currently, three different IMNM entities have been defined: 1) anti-3-hydroxy-3-methylglutaryl-coenzyme A reductase (HMGCR) myopathy; 2) anti-signal recognition particle (SRP) myopathy; 3 ) antibody negative IMNM. An accurate diagnosis of IMNMs is relevant for prognostic purposes and to provide the best chance of treatment for patient subtypes and prevent long-term disability. 
We aimed to investigate clinical and histological features of different serological subgroups within an Italian cohort of IMNM patients.

We included 124 patients diagnosed with IMNM in 12 neuromuscular referral centers in Italy, relying on the 2017 European Neuromuscular Centre criteria. The study population was composed by 54 males and 70 females, with an overlapping median age at disease onset equal to 64 years old for men and 65 for women. Forty-five patients $(36,3 \%)$ were positive for HMGCR autoantibodies (Abs), of whom 29 had a history of statin exposure (13males, 16 females). Among anti-HMGCR Ab-positive IMNM patients naïve to statin therapy, females were more represented $(62,5 \%)$. Further, 35 patients $(28,2 \%)$ had anti-signal recognition particle (SRP) Abs, 24 patients $(19,4 \%)$ resulted seronegative, and 20 patients $(16,1 \%)$ did not have a complete $\mathrm{Ab}$ assessment. Malignancies were reported in 16 patients. This study provides new insights about IMNM characteristics in a large Italian cohort.

\section{Genilam, an Italian Project to shorten the time of molecular diagno-} sis for ATTR amyloidosis patients

D. Bonvissuto ${ }^{1}$, E.Rizzo $^{1}$, F. Franchini ${ }^{1}$, A. Nuccitelli ${ }^{1}$, A. Biricik ${ }^{1}$, C. Grillo $^{1}$, L. Barbetta ${ }^{1}$, F. Fiorentino ${ }^{1}$.

${ }^{1}$ Molecular Genetics Laboratory, Rome, Italy.

Hereditary Transthyretin amyloidosis (hATTR) is a potentially lifethreatening condition characterized by extracellular deposition of amyloid fibrils composed of transthyretin (TTR) with an altered structure. The TTR gene, when mutated, codes for a protein with a tendency to aggregate into amyloid fibrils, resulting in progressive motor and sensory neuropathy with cardiac involvement, and possible ocular and renal complications. As part of the Italian project called Genilam, clinical centers were offered a genetic test service at no charge with the aim of shortening the time of molecular diagnosis and identifying the disease early in order to slow its progression.

The analysis was performed by gene amplification (PCR) of exons 2 , 3 and 4 of the TTR gene, and subsequent automated sequencing (ABI Prism 3130XL) for the investigation of the main mutations. In the first 12 months of the project, about 300 specialized medical centers were involved, and 552 tests were performed, of which 46 were positive $(8.3 \%)$. The most common mutations identified in positive patients were Val50Met and Phe84Leu. The diagnosis of symptomatic positive subjects has, in some cases, also allowed the subsequent intra-family identification of asymptomatic carriers for monitoring and timely management of the disease. The early molecular diagnosis of mutations on the TTR gene is essential to improve the prognosis of the disease. The Genilam project has helped in Italy to rapidly identify many more patients and asymptomatic carriers than in the past, thanks also to the great consensus received by the specialized healthcare professionals

Clinical, morphological and genetic data of patients with distal and myofibrillar myopathies: report from the Italian network

S. Bortolani ${ }^{1,10}$, S. Bonanno ${ }^{4}$, G. Vattemi ${ }^{2}$, P. Tonin ${ }^{2}$, M. Monforte $^{1}$, E. Ricci $^{1}$, G. Primiano ${ }^{1}$, S. Servidei ${ }^{1}$, G. Greco ${ }^{3}$, R. Massa ${ }^{3}$, C. Gemelli ${ }^{5}$, M. Grandis $^{5}$, C. Fiorillo ${ }^{6}$, A. Petrucci ${ }^{7}$, M. Filosto ${ }^{8}$, M.L. Valentino', R. Liguori $^{9}$, T. Mongini ${ }^{10}$, M. Garibaldi ${ }^{11}$, G. Antonini ${ }^{11}$, M. Lucchini ${ }^{1}$, M. Mirabella ${ }^{1}$, A. Rubegni ${ }^{12}$, F. M. Santorelli ${ }^{12}$, G. Siciliano ${ }^{13}$, G. Ricci $^{13}$, C. Angelini ${ }^{14}$, A. Ariatti ${ }^{15}$, L. Maggi ${ }^{4}$, G. Tasca ${ }^{1 *}$

${ }^{1}$ Unità Operativa Complessa di Neurologia, Fondazione Policlinico Universitario A. Gemelli IRCSS, Rome, Italy;
${ }^{2}$ Dipartimento di Neuroscienze, Biomedicina e Disturbi del Movimento, Università di Verona, Verona, Italy

${ }^{3}$ Neuromuscular Diseases Unit, Department of Systems Medicine, University of Rome Tor Vergata, Rome, Italy.

${ }^{4}$ Foundation IRCCS Neurological Institute Carlo Besta, Milan, Italy

${ }^{5}$ Department of Neurosciences, Rehabilitation, Ophthalmology, Genetics and Maternal/Child Sciences, University of Genoa, Italy.

${ }^{6}$ Pediatric Neurology and Neuromuscular Disorders, Istituto G. Gaslini and University of Genoa, Italy.

${ }^{7}$ Center for Neuromuscular and Neurological Rare Diseases S. Camillo Forlanini Hospital, Rome, Italy.

${ }^{8}$ Unit of Neurology, ASST "Spedali Civili" and University of Brescia, Brescia, Italy

${ }^{9}$ IRCCS Istituto delle Scienze Neurologiche di Bologna, UOC Clinica Neurologica, Bologna, Italy

${ }^{10}$ Department of Neurosciences Rita Levi Montalcini, University of Torino, Torino, Italy.

11 Neuromuscular and Rare Disease Center, Department of Neuroscience, Mental Health and Sensory Organs (NESMOS), SAPIENZA University, Sant'Andrea Hospital, Rome, Italy

${ }_{12}$ Molecular Medicine for Neurodegenerative and Neuromuscular Diseases Unit, IRCCS Stella Maris Foundation, Pisa, Italy.

${ }^{13}$ Department of Clinical and Experimental Medicine, Neurological Clinic, University of Pisa, Italy

${ }^{14}$ Neuromuscular Center, IRCCS San Camillo Hospital, Venice, Italy

${ }^{15}$ Department of Biomedical, Metabolic, Neural Sciences, University Hospitals of Modena, Italy.

* These authors contributed equally to the work

Introduction: Myofibrillar myopathies (MFM) are muscle diseases with typical histopathology of myofibrillar network disruption and abnormal protein deposition. Distal myopathies are a genetically heterogeneous group of muscle disorders with prevalent weakness in the distal part of upper and lower limbs. Given their overlapping features, the boundaries between the two entities are uncertain. In 2018 the Italian network of distal and myofibrillar myopathies was established.

Methods: Patients with either a pathological diagnosis of MFM or a clinical diagnosis of distal myopathy were included. Both molecularly confirmed patients and subjects still without an identified gene defect were included. Clinical, instrumental, genetic, and pathological data was collected.

Results: Fifteen Italian neuromuscular centers joined the network. Information on more than two hundred patients was retrospectively acquired. Eighty $\%$ of the patients had a distal myopathy phenotype. About $30 \%$ had MFM pathology. The most frequent pathological abnormality (30\% of the patients) was the presence of rimmed vacuoles, either isolated or combined with MFM features. About half of the subjects had a molecular diagnosis. Mutations in 18 different genes were identified; the most prevalent were DNAJB6, MYOT and DES. Cardiac involvement was present in $20 \%$ of the patients, mainly in patients carrying $D E S$ mutations.

Conclusions: We present the clinicopathological and genetic findings of a large population of distal and myofibrillar myopathies. Approximately half of the patients are still molecularly undiagnosed despite extensive search for pathogenic mutations. Collaborative efforts are mandatory to collect and deeply characterize these patients with the aim of identifying new disease-related genes.

Light-Chain Neurofilaments (Nf-L) assessment in type 3 Spinal Muscular Atrophy patients treated with nusinersen

V. Bozzoni ${ }^{1}$, G. Musso ${ }^{2}$, L. Caumo ${ }^{1}$, L. Bello ${ }^{1}$, S. Tripodi ${ }^{1}$, P. Riguzzi ${ }^{1}$, F. Causin ${ }^{2}$, J. Gabrieli ${ }^{3}$, G. Cester ${ }^{3}$, G. Sorarù ${ }^{1}$, M. Plebani ${ }^{2}$, E. Pegoraro ${ }^{1}$. 
${ }^{1}$ Department of Neurosciences, University Hospital of Padua, Padua, Italy.

${ }^{2}$ Department of Laboratory Medicine, University Hospital of Padua, Padua, Italy.

${ }^{3}$ Neuroradiology Unit, University Hospital of Padua, Padua, Italy.

Aim: The advent of nusinersen treatment for Spinal Muscular Atrophy (SMA) patients is deeply modifying disease natural history in infantile patients and is under investigation in adult patients. Validated clinical outcome measures are needed, but also the identification of biological biomarkers, like neurofilaments ( $\mathrm{Nfs}$ ), is urgent.

The aim of this study was to determine whether cerebrospinal fluid (CSF) light-chain neurofilament (NfL), total Tau (t-Tau) and Tau phosphorylated (p-Tau 181) can be used as biomarker of neuronal damage and/or of therapeutic response-biomarker in adult nusinersen-treated SMA patients. Materials and methods: We recruited 30 adult SMA3 patients who were referred to the Neuromuscular Center of Padua Hospital for nusinersen treatment from February 2018 and September 2019. CSF NfL, t-Tau and p-Tau 181were analyzed at each therapy infusion until the third maintenance administration. Baseline NfL were compared with a control group. Results: Baseline CSF NfL, t-Tau and p-Tau levels were in the reference ranges, except from NfL values in one patient only. Mean NfL were lower in patients $(218.23 \pm 188.40 \mathrm{ng} / \mathrm{l})$ compared controls $(809.53 \pm 1,065.26$ $\mathrm{ng} / \mathrm{l}$ ), correlated with age both in patients and controls and, among patients, were not correlated with clinical severity, or with $S M N 2$ copy number. Longitudinal CSF biomarker analysis showed a statistically significant increase during nusinersen loading phase, without correlation with clinical outcome measures, and thereafter a trend towards basal values.

Discussion and conclusion: Our data suggest that CSF NfL are not a disease biomarker in adult SMA. The increase in the loading phase needs further studies to be fully understood.

\section{Neuropsychological profile of Becker Muscular Dystrophy}

R. Brusa ${ }^{1}$, F. Magri ${ }^{1}$, T. Difonzo ${ }^{2}$, D. Velardo ${ }^{4}$, S. Corti ${ }^{3}$, M. Moggio ${ }^{4}$, M.C. Saetti ${ }^{2}$, G.P.Comi ${ }^{3,4}$

${ }^{1}$ Dino Ferrari Centre, Neuroscience Section, Department of Pathophysiology and Transplantation, IRCCS Foundation Ca' Granda Ospedale Maggiore Policlinico, Milan,

${ }^{2}$ Neurology Unit, IRCCS Foundation Ca' Granda Ospedale Maggiore Policlinico, University of Milan, Milan

${ }^{3}$ Dino Ferrari Centre, Neuroscience Section, Department of Pathophysiology and Transplantation, IRCCS Founsdation Ca' Granda Ospedale Maggiore Policlinico, University of Milan, Milan;

${ }^{4}$ Neuromuscular and Rare Disease Unit, Department of Neuroscience, Foundation IRCCS Ca' Granda Ospedale Maggior e Policlinico, Dino Ferrari Centre, University of Milan, Milan

Becker muscular dystrophy (BMD) is an heterogeneous neuromuscular disorder due to mutations in the $D M D$ gene, characterized by progressive proximal muscles weakness, calf hypertrophy and cardiomyopathy. Different isoforms of the dystrophin are also expressed in cerebral cortex and Purkinje cells. Studies on Duchenne Muscular Dystrophy revealed lower scores in verbal intellective quotient (IQ), verbal working memory and verbal understanding abilities. Impairment in language, short term memory, reading, writing and executive functions has also been reported. Since now only few data about CNS involvement in BMD are available. We collected clinical history and sociological information and performed extensive neuropsychological assessments on 28 male BMD patients with age from 18 to 65 years old, ambulant and without a relevant cardiomyopathy. Most of the patients carried a deletion of exons 45-47. Cognitive aspects analysed included attention and executive function, deductive reasoning, language, memory, praxis and pre-frontal functions.
None of the patients showed intellectual deficiency. Significant abnormalities were detected mainly with the Dual Task (11/28) and PASAT $(6 / 28)$ tests. Borderline scores have also been reported in the Recurrent Faces (1 pathological, 10 borderline), Inverse Digit Span (1 pathologic, 8 borderline) and Inverse Corsi Span (3 pathologic, 3 borderline) tests.

Despite cognitive abnormalities in DMD subjects have been described in literature, little is known about a comprehensive cognitive profile in BMD patients. In this study, the pattern of cognitive impairment points toward a defect in working memory in the BMD population. This data need confirmation with further evaluation in a wider and genetically heterogeneous population.

\section{Epidemiological study of HEV prevalence in patients with CIDP and ALS}

C. Cambieri ${ }^{1}$, M. Ceccanti ${ }^{1}$, L. Libonati ${ }^{1}$, I. Fiorini ${ }^{1}$, V. Frasca ${ }^{1}$, E. Onesti ${ }^{1}$, G. Taliani ${ }^{2}$, M. Inghilleri ${ }^{1}$

${ }^{1}$ Rare Neuromuscular Diseases Center, Department of Human Neuroscience, Sapienza University of Rome, Rome, Italy

${ }^{2}$ Chronic Infectious Diseases Unit, Policlinico Umberto I, Sapienza University of Rome, Rome, Italy

Hepatitis E virus (HEV) infection is an emerging autochthonous disease in industrialized countries. Neurologic symptoms have been reported in up to $5 \%$ of patients with an $\mathrm{HEV}$ infection, indicating that HEV could have a specific neurotropism. In a previous study, italian blood donors showed a prevalence of anti-HEV IgG of $8,7 \%$. Here we describe the HEV prevalence in patients with definite CIDP, definite ALS and ALS caregivers. We enrolled 45 patients with CIDP, 96 patients with ALS and 75 healthy subjects. Each subject underwent a clinical evaluation, including a survey which collected demographic and anamnestic variables, lifestyle and eating habits. Each subject then underwent a blood sample to determine the serological assessment for HEV (anti-HEV IgG, IgM) and HEV-RNA. In ALS group, anti-HEV IgG prevalence was $18,75 \%$; in line with their caregivers who showed a prevalence of $20,0 \%$. Anti-HEV IgM prevalence was $4,16 \%$. All the samples were negative for HEV-RNA. In the CIDP group, anti-HEV IgG was 33,33\%. All CIDP subjects resulted negative for anti-HEV IgM and HEV-RNA. We calculated the odds ratio of anti-HEV IgG prevalence of ALS/CIDP patients and of the general population (age and sex-matched blood donors); we found a significant difference both for the ALS (OR 2.34 $p=0,014$ ) and the CIDP group (OR $5.07-p=0,000009$ ). Our results shows that anti-HEV IgG prevalence is significantly higher in ALS and CIDP patients than in the control group, thus demonstrating a possible association between a previous HEV infection and the neurological disorders.

Arsenic Trioxide-induced peripheral neuropathy: prospective evaluation in a cohort of patients with acute promyelocytic leukemia

M. Campagnolo ${ }^{1}$, F. Lessi ${ }^{2}$, M. Cacciavillani ${ }^{3}$, M. Riva ${ }^{2}$, A. Salvalaggio $^{1}$, F. Castellani ${ }^{1}$, C. Briani ${ }^{1}$.

${ }^{1}$ Department of Neuroscience, University of Padova

${ }^{2}$ Hematology and Clinical Immunology Unit, Department of Medicine, University of Padova

${ }^{3}$ CEMES, Synlab Group, Padova, Italy

Arsenic trioxide (ATO), effective in acute promyelocytic leukemia (APL), has several side effects (QT prolongation, hepatotoxicity), but less is known regarding peripheral neurotoxicity. 
We prospectively evaluated APL patients undergoing ATO as first-line therapy at baseline, end of the induction and consolidation. The Total Neuropathy Score clinical version (TNSc), a validated measure for chemotherapy-induced peripheral neurotoxicity, was used, with TNSc $>2$ considered significant for neuropathy. Neurophysiology at the end of induction was performed in 10/14 patients.

Fourteen patients ( 9 men, mean age 57.3 \pm 13.7 , range $37-81$ yrs) were enrolled. Median TNSc at baseline was 0 , except in one patient presenting reduced ankle reflexes and distal paresthesias (TNSc 3). During induction, 11/14 patients developed clinical neuropathy (median TNSc 2.5, range $0-8$ ). Sensory impairment, paresthesias and neuropathic pain were the most common complaints; muscle weakness was reported in 3 patients. Neurophysiology at the end of induction revealed axonal peripheral neuropathy in 6/10, and demyelinating features consistent with hereditary neuropathy in the patient with TNSc 3 at baseline. To date, consolidation has been completed in $8 / 14$ patients. Seven patients complained of neuropathic symptoms with $6 / 7$ improving of at least 1 point in TNSc and 2 reporting TNSc 0 . In 2 patients with 1-year followup, full clinical and neurophysiological recovery was observed.

Patients undergoing ATO therapy for APL may develop sensory axonal neuropathy in the early stages of induction, that seems to improve up to recovery during follow-up. A multicenter prospective study evaluating the occurrence and risk factors of ATO-induced neuropathy is ongoing.

\section{Neurolymphomatosis as the main presentation of relapse of extranodal diffuse large B-cell lymphoma (DLBCL)}

M. Campagnolo ${ }^{1}$, M. Cacciavillani ${ }^{2}$, T. Cavallaro ${ }^{3}$, S. Ferrari $^{3}$, L. Pavan ${ }^{4}$, G. Barilà ${ }^{4}$, A. Salvalaggio ${ }^{1}$, F. Castellani ${ }^{1}$, R. Zambello ${ }^{4}$, C. Briani ${ }^{1}$.

${ }^{1}$ Department of Neurosciences, University of Padova, Padova

${ }^{2}$ CEMES-EMG Lab, Synlab Group, Padova

${ }^{3}$ Section of Neurology, Department of Neurosciences, Biomedicine and Movement Sciences, University of Verona, Italy

${ }^{4}$ Hematology and Clinical Immunology Unit, Department of Medicine, University of Padova, Padova

Neurolymphomatosis (the histologically documented infiltration of the peripheral nervous system), is an uncommon manifestation of lymphomas. We report on a 66-yr-old woman with extranodal diffuse large Bcell lymphoma (DLBCL), treated successfully (2002-2003) with radiation and chemotherapy.

In 2011, the patient complained of impaired sensation, neuropathic pain and weakness at left abdomen and thigh. Neurophysiology showed left T10-L4 radiculo-plexopathy, with no abnormalities at cerebrospinal fluid (CSF) analysis. MR-Neurography disclosed severe atrophy of the left rectus abdominis muscle, neurogenic edema and denervation. Despite no hematological findings, a possible neurolymphomatosis was suspected, and the patient treated with rituximab until August 2013, with improvement. In March 2014, right leg weakness occurred with neurophysiology showing right L3-L4 radiculo-plexopathy, absent sensory action potential of the superficial peroneal nerve. The patient refused nerve biopsy and rituximab was administered with partial response. The patient relapsed again in December 2018 (right foot drop) with neurophysiology disclosing L4-L5 and L5-S1 radiculo-plexopathy, decreased amplitude of the right sural nerve, increased cross-sectional area of the common peroneal and right sciatic nerves at nerve ultrasound. CSF was unremarkable. Sural nerve biopsy detected axonal neuropathy with fascicular asymmetry, endoneurial infiltrates with B-lymphoid elements. Neurolymphomatosis was diagnosed and rituximab plus bendamustine administered for 4 cycles. In November 2019, cutaneous nodules tested positive for lymphoma cells, and salvage RDHAOx (rituximab, dexamethasone, oxaliplatin, cytarabine) was performed before autologous stem cell transplant. Despite unremarkable hematological findings, neurolymphomatosis should be considered in patients with lymphoma and unexplained peripheral nervous system involvement.
Sphingomyelin: a novel diagnostic and disease activity biomarker for the management of acquired demyelinating neuropathies

G. Capodivento ${ }^{1,2}$, C. De Michelis ${ }^{1,2}$, M. Carpo ${ }^{3}$, R. Fancellu ${ }^{4}$, E. Schirinzi $^{5}$, D. Severi ${ }^{6}$, D. Visigalli ${ }^{1,2}$, D. Franciotta $^{7}$, G. Siciliano ${ }^{5}$, F. Manganelli $^{6}$, A. Beronio ${ }^{8}$, E. Capello ${ }^{1,2}$, P. Lanteri' ${ }^{9}$, E. Nobile-Orazio ${ }^{10}$, A. Schenone ${ }^{1,2}$, L. Benedetti $^{1,2}$, L. Nobbio ${ }^{1,2}$

${ }^{1}$ DINOGMI - Università di Genova, Italia

${ }^{2}$ IRCCS Ospedale Policlinico San Martino, UO Clinica Neurologica, Genova, Italia

${ }^{3}$ UOC Neurologia Treviglio, ASST Bergamo Ovest, Italia

${ }^{4}$ IRCCS Ospedale Policlinico San Martino, UO Neurologia, Genova, Italia

${ }^{5}$ Dipartimento di medicina e clinica sperimentale, Clinica Neurologica, Università di Pisa, Italia

${ }^{6}$ Azienda Ospedaliera Universitaria Federico II, Napoli, Italia

${ }^{7}$ IRCCS Fondazione Mondino, Pavia, Italia

${ }^{8}$ Ospedale Sant'Andrea, La Spezia, Italia

${ }^{9}$ IRCCS Istituto Neurologico Carlo Besta, Milano, Italia

${ }^{9}$ Dipartimento di Neurologia, Università di Milano - IRCCS Humanitas, Rozzano (MI), Italia

Finding biomarkers to support diagnosis, monitor disease activity, treatment response and duration is a priority for the GBS/CIDP community. Published guidelines improve diagnostic accuracy but are often underutilized during routine clinical practice. We have already demonstrated that sphingomyelin (SM), a myelin-enriched lipid, is extremely higher in the CSF of GBS/CIDP patients.

To definitively validate SM as myelin biomarker relevant for the management of these patients, we planned an Italian multicentre prospective study on a large number of clinically and neurophysiologically wellcharacterized patients.

We confirmed that SM is significantly higher in the CSF of patients affected by typical $\operatorname{CIDP}(n=35)$, atypical CIDP $(n=18)$ and $\operatorname{AIDP}(n=12)$ compared to patients affected by non-demyelinating diseases used as controls $(\mathrm{n}=85)$ $\left(\mathrm{p}=1.3^{*} 10^{-12}, \mathrm{p}=6.5^{*} 10^{-3}\right.$ and $\mathrm{p}=3.4 * 10^{-5}$, respectively). Actually, to test the good performance of SM as a myelin biomarker we ran a ROC curve analysis obtaining an AUC of 0.9447 with a sensitivity of $80.85 \%$ and a specificity of $98.82 \%$. Moreover, SM identifies disease activity in CIDP patients applying for a selective tool to treatment tailoring or withdrawal. Finally, SM correlates with the main clinical scores (ONLS, MRC, INCAT, GBS disability scale) and some neurophysiological parameters in CIDP and AIDP patients. Notably, CSF SM is more specific than CSF protein concentration to finalize a correct diagnosis for suspected CIDP and can be very useful to avoid misdiagnosis.

Overall, our study demonstrates that SM dosage can be used as a routine CSF analysis to effectively improve the management of GBS/CIDP patients.

\section{Clinical presentations of two rare mutations of $T T R$}

D. Cardellini ${ }^{1}$, F. Taioli ${ }^{1}$, M. Cappellari ${ }^{2}$, M. Milan ${ }^{3}$, L. Bertolasi ${ }^{4}$, G.M. Fabrizi $^{1,4}$, T. Cavallaro ${ }^{4}$

${ }^{1}$ Section of Neurology, Department of Neurosciences, Biomedicine and Movement Sciences, University of Verona

${ }^{2}$ Section of Neurology A, Department of Neurosciences, AOUI Verona

${ }^{3}$ Neurology Unit, Mirano Hospital, Mirano, Venezia

${ }^{4}$ Section of Neurology B, Department of Neurosciences, AOUI Verona

Hereditary Transthyretin Amyloidosis (hATTR) has three main presentations: progressive sensorimotor polyneuropathies often associated with carpal tunnel syndrome (CTS), autonomic neuropathy and infiltrative cardiomyopathy. Val30Met and Phe64Leu (manifesting as late-onset sensori-motor polyneuropathy), and Ile68Leu (associated with cardiomyopathy) are the 
most frequent mutations in Italy. We report two pedigrees carrying rare TTR mutations presenting without overt family history.

A female proband died at age 78 because of severe restrictive cardiomyopathy. Since the sixth decade she had suffered from visual disturbances due to the outcomes of bilateral vitrectomy. By age 72 years she had presented a progressive sensori-motor-autononomic polyneuropathy. The proband and two asymptomatic daughters, aged 45 and 43 years, harboured a p.Ala36Pro substitution. Significantly, the same mutation had been reported in a singleton pedigree and associated with amyloid-related vitreous opacities as heralding feature of hATTR (Jones, Am J Hum Genet 1991).

A 67-year-old female proband presented recurrent ischemic strokes associated with a restrictive cardiomyopathy without atrial fibrillation or dilatation. RMN was consistent with cardioembolic strokes and genetic testing demonstrated a Glu62Lys substitution which had been previously reported in a singleton case with TTR-related cardiomyopathy initially misdiagnosed as AL amyloidosis (Briani, J Neurol 2012). Two sisters aged 61 and 65 years, carried the same mutation and were asymptomatic with the exception of a bilateral CTS.

The report highlights the heterogeneity of genotype-to-phenotype correlations and variable scenarios of neurological involvement in hATTR.

\section{Ocrelizumab in a case of refractory Chronic Inflammatory} Demyelinating Polyneuropathy (CIDP) with antibodies anti Rituximab

S. Casertano ${ }^{1}$; E. Signoriello; ${ }^{1}$; F Rossi ${ }^{1}$; A Di Pietro; F Tuccillo ${ }^{2}$; S Bonavita $^{1}$, G. Lus ${ }^{1}$

${ }^{I}$ Multiple Sclerosis Center; II division of Neurology; University of Study Luigi Vanvitelli

${ }^{2}$ UOC Neurofisiopatologia AORN Cardarelli, Naples, Italy.

Background: Chronic inflammatory demyelinating polyradiculoneuropathy (CIDP) is a chronic autoimmune inflammatory disease affecting peripheral nervous system. Ocrelizumab (OCR) is humanized anti-CD20 antibodies now approved for Multiple Sclerosis that could have higher tolerability and lesser immunogenic profile compared to RTX. We present a case of refractory CIDP treated effectively with Ocrelizumab switched from RTX due to resistance for development of antibodies.

Case report: A 24 years old young man was admitted for the first time at our clinic for onset of distal muscle weakness and distal numbness of all limbs. Clinical and instrumental findings met the definite category of electrodiagnostic criteria for CIDP. After acute therapies with IVIG and steroids, the patient started firstly with inefficacy Azathioprine and after RTX with post infusional general malaise and arthralgias and development of high title of antibodies (1:18000). An off-label treatment with OCR was started due to less immunogenicity, with good clinical response and absence of adverse events.

Discussion and Conclusion: In our case, patient had a good clinical response with OCR treatment after biological resistance to RTX. We suggest that OCR can be consider as another therapeutic chance in refractory CIDP. In literature this is the first case of CIDP treated with OCR, and the first case demonstrating the possibility of using OCR after Rituximab obtaining anti-CD20 effect due to different immunogenicity.

\section{Serum neurofilament light chain (sNfL) correlate with sural nerve pathological findings}

\footnotetext{
F. Castellani ${ }^{1}$, S. Mariotto ${ }^{2}$, M. Campagnolo ${ }^{1}$, S. Ferrari ${ }^{2}$, C. Briani ${ }^{1}$

${ }^{1}$ Department of Neurosciences, University of Padova, Padova

${ }^{2}$ Section of Neurology, Department of Neurosciences, Biomedicine and Movement Sciences, University of Verona
}

The aim of our study was to investigate the role of serum neurofilament light chain (sNfL) as a marker of disease activity and therapy response in chronic inflammatory demyelinating polyradiculoneuropathy (CIDP). Correlation with neuropathological data from available sural nerve biopsy was also performed.

Sera from 14 patients ( 12 men, 2 women; mean age $58.14 \pm 15.57$ years; mean sNfL levels $32.07 \pm 34.26 \mathrm{pg} / \mathrm{mL}$ ) fulfilling the clinical and neurophysiological EFNS/PNS criteria for definite CIDP were considered. In 10 patients a follow-up sample was collected after a mean of $18.2 \pm 13.42$ months of therapy. The severity of neuropathy and the response to treatment were assessed with the INCAT (Inflammatory Neuropathy Cause and Treatment) Disability Scale. Five patients underwent sural nerve biopsy at the same time as the first blood sample.

As control, 6 patients ( 3 men, 3 women; mean age $71.33 \pm 6.89$ years; mean sNfL levels $37.83 \pm 51.78 \mathrm{pg} / \mathrm{mL}$ ) with axonal neuropathy, one with motor neuron disease and 14 age-matched healthy controls (mean age $57.1 \pm 4.2$ years; mean sNfL levels $9.6 \pm 2.91 \mathrm{pg}$ / $\mathrm{mL}$ ) were included.

With regard to the correlation with pathological findings, we observed higher sNfL levels in cases with a mixed $v s$ demyelinating pattern at sural nerve biopsy, that correlated with the amount of fiber loss and axonal degeneration, while the percentage of demyelination, the presence of inflammatory infiltrates, onion bulbs, and complement/Ig deposits did not influence sNfL values. Data from sNfL and therapy response were instead less consistent, with sNfL values changing more significantly only in few patients with dramatic clinical improvement or worsening.

Mutational profile in patients with anti-myelin-associated glycoprotein (MAG) antibody neuropathy identifies new therapeutic target

F. Castellani ${ }^{1}$, A. Visentin ${ }^{2}$, M. Campagnolo ${ }^{1}$, A. Salvalaggio ${ }^{1}$, C. Candiotto $^{3}$, R. Zambello ${ }^{2}$, F. Piazza ${ }^{2}$, L. Trentin' ${ }^{2}$, R. Bertorelle ${ }^{3}$, C. Briani $^{1}$

${ }^{1}$ Department of Neurosciences, University of Padova, Padova

${ }^{2}$ Hematology and Clinical Immunology Unit, Department of Medicine, University of Padova, Padova

${ }^{3}$ Immunology and Molecular Oncology, Veneto Institute of Oncology IOV -IRCCS, Padova

Anti-myelin-associated glycoprotein (MAG) antibody neuropathy is associated either with IgM monoclonal gammopathy of undetermined significance (MGUS) or lymphoproliferative disorder (Waldenstrom's Macroglobulinemia [WM], marginal zone lymphoma [MZL] or chronic lymphocytic leukemia [CLL]). Ibrutinib, an oral inhibitor of Bruton's tyrosine kinase, has been shown to be effective in WM, especially with $M Y D 88^{L 265 P}$ mutated and CXCR4 $4^{S 338 X}$ wild-type.

In a single center study, we assessed the mutational profile of the MYD 88 and $C X C R 4$ genes of 26 patients ( 8 women, 18 men, mean age $73.14 \pm 7$ years; 9 previously treated with rituximab) with anti-MAG antibody neuropathy. Of them, 13 (50\%) had IgM-MGUS, 10 (38\%) WM, 3 (12\%) CLL/MZL. Molecular analysis was performed from bone marrow mononuclear cells, using allele specific-PCR. Seventeen patients $(65 \%)$ carried the MYD $88^{L 265 P}$ mutation: all the $10 \mathrm{WM}$ patients, $6 / 13$ (46\%) MGUS and $1 / 3(33 \%)$ CLL/MZL. All the patients were CXCR $4^{S 338 X}$ wild-type. Three patients (all MYD $88^{L 265 P}$ mutated and CXCR $4^{S 338 X}$ wild-type) were treated with ibrutinib $420 \mathrm{mg} /$ die orally. Patients were assessed at baseline, and every 3 months up to 12 months, using INCAT (Inflammatory Neuropathy Cause and Treatment) Disability Score, INCAT Sensory Sum Score, Medical Research Council sum score and Modified International Cooperative Ataxia Rating Scale. All the patients reported an early and progressive improvement, especially of the sensory symptoms as shown by improvement of clinical scales. Treatment was well tolerated. 
The molecular study helped identify the presence of a mutational target for a new and effective therapy, ibrutinib, for anti-MAG antibody neuropathy, where rituximab is effective in only half of the patients.

\section{Pilot study in phenotypic variability of cardiac involvement in a co-} hort of patients with Becker muscular dystrophy

V. Castiglione ${ }^{1}$, G. Ricci ${ }^{2}$, A. Govoni ${ }^{2}$, G. Astrea ${ }^{3}$, A. Rocchi ${ }^{2}$, F. Baldinotti ${ }^{4}$, A. Giannoni ${ }^{5}$, C. Passino ${ }^{5}$, M. Emdin ${ }^{5}$, G. Siciliano ${ }^{2}$

${ }^{1}$ Scuola di specializzazione in malattie dell'apparato cardiovascolare, Università di Pisa, Pisa, Italy

${ }^{2}$ Department of Clinical and Experimental Medicine, University of Pisa, Pisa, Italy

${ }^{3}$ Department of Developmental Neuroscience, IRCCS Stella Maris, Calambrone (Pisa), Italy

${ }^{4}$ Laboratory of Medical Genetics, Azienda Ospedaliero-Universitaria Pisana, S. Chiara Hospital, Pisa, Italy

${ }^{5}$ Cardiology Division, Fondazione Toscana Gabriele Monasterio, Pisa, Italy

Becker muscular dystrophy (BMD) is characterized by a broad phenotypic spectrum. We propose a detailed clinical protocol and molecular analysis aimed to describe a cohort of BMD patients. We recruited 30 adult BMD subjects, stratified based on the type of mutation and degree of muscular impairment assessed by functional motor scales and qualitative analysis of muscle magnetic resonance imaging (MRI). Muscular MRI is useful to recognize a specific pattern of involvement and to follow longitudinally the disease progression. We confirmed the correlation between genotype and muscular involvement. Moreover, a comprehensive assessment of cardiac impairment was performed on 10 BMD patients, aged $39 \pm 19$ years, analyzing cardiac MRI and blood biomarkers of cardiac and muscular damage. In the studied cohort, there was no clear correlation between genotype and cardiac phenotype; myocardial fibrosis as assessed by late gadolinium enhancement (LGE) was present in six patients and three patients demonstrating reduced left ventricular ejection fraction. LGE-positive patients showed a trend towards higher values of high-sensitivity troponin $\mathrm{T}$ (hs-TnT) and I (hsTnI), N-terminal fraction of pro-B-type natriuretic peptide (NTproBNP) and norepinephrine, although only hs-TnT difference reached statistical significance $(p=0.019)$. Notably, levels of the more cardiac-specific hs-TnI were always below the cut-off, while hs-TnT levels were above the $99^{\circ}$ percentile in $60 \%$ of patients and well correlated with myoglobin $(r=0.953, p<0.001)$ and creatine kinase $(r=0.615, p=0.059)$. hs-TnT is significantly higher in LGEpositive patients, but also correlates with biomarkers of muscular damage making it a sensitive marker of both myocardial and muscular damage in BMD.

\section{Mutations in ASCC3 are associated with severe congenital myopathy, arthrogryposis and bone fractures}

M. Catteruccia ${ }^{1}$, D. Diodato ${ }^{1}$, F. Fattori ${ }^{1}$, G. Colia $^{1}$, F. De Mitri ${ }^{1}$, A. D’Amico $^{1}$, E. Bertini ${ }^{1}$

${ }^{1}$ Unit of Neuromuscular and Neurodegenerative Disorders, IRCCS Bambino Gesù Childrens' Research Hospital, Rome

Background:ASCC 3 encodes one of the four subunits of the activating signal cointegrator 1 (ASC-1) complex, composed of ASCC1, ASCC2, ASCC3 and TRIP4, that acts as a transcriptional cointegrator complex and participates in RNA processing events. So far, only mutations in
ASSC1 and TRIP 4 have been associated with severe muscle and bone disorders.

Objective: We report the identification of mutations in $A S C C 3$ presenting as prenatal onset muscle weakness, arthrogryposis and bone fractures.

Methods: We describe clinical, histological, genetic and functional data of a baby with mutations in ASCC3.

Results: The patient is a boy with intrauterine growth retardation, oligo/ anhydramnios and fetal hypo/akinesia. At birth he presented generalized hypotonia and muscle weakness, arthrogryposis, diffuse osteopenia and bone fractures, cleft palate, dysphagia reguiring nasogastrig tube feeding and respiratory distress with intubation and mechanical ventilation in the second day of life.

Muscle biopsy showed severe myopathic changes with marked variability in fibers size, internalized nuclei, increased connective tissue and predominance of type 1 fibers. Exome sequencing identified a compoud heterozigosity for the nonsense c.5281C $>\mathrm{T}$ (p.Arg1761Ter) and missense c.4955G $>\mathrm{A}$ (p.Gly1652Asp) mutations in ASCC3. Transcriptional analysis documented altered ASCC 3 mRNA levels in the patient compared to controls.

Conclusion: Our study shows that mutations in ASCC 3 are associated with severe muscle phenotype with arthrogryposis and congenital bone fractures. The clinical and histological picture of our patient is very similar to that reported in association of ASCCland further underlies the importance of ASC-1 complex in the development of muscle and bone structures.

\section{Early onset recurrent muscle dysfunction associated with novel} POGLUT1 mutation

A. Cavaliere ${ }^{1}$, S. Gibertini ${ }^{1}$, A. Ruggieri ${ }^{1,3}$, F. Blasevich ${ }^{1}$, F.R. Danti ${ }^{2}$, L. Maggi ${ }^{1}$, I. Moroni ${ }^{2}$

${ }^{1}$ Neuromuscular Diseases and Neuroimmunology Unit - Fondazione IRCCS Istituto Neurologico C. Besta, Milano, Italy.

${ }^{2}$ Department of Pediatric Neurosciences - Fondazione IRCCS Istituto Neurologico C. Besta, Milano, Italy.

${ }^{3}$ Department of Molecular and Translation Medicine, Unit of Biology and Genetics, University of Brescia, Brescia, Italy.

Mutations in Protein $O$-glucosyltransferase1 (POGLUT1) gene have been recently described in families displaying clinical picture of limb girdle muscle weakness with variable presentation, from congenital to adult onset (LGMD R21; OMIM\# 617232).

POGLUT1 activity is critical for the Notch signalling pathway since addition of O-glucose glycans to Notch is required for its proper trafficking to the cell surface in multiple tissues, including skeletal muscle.

We describe a patient presenting since the age of 17 months with several recurrent episodes of myalgia and generalized muscle weakness, leading to loss of autonomous walking, and with resolution in a few days. Symptoms were not related to exercise or fasting or infections. CPK resulted elevated during the episodes $(10.000 \mathrm{IU} / \mathrm{l})$ and also in the period of well-being (5.000 IU/1).

At neurological examination between the crises the child showed no signs of myopathy, raising the suspicion of a metabolic myopathy. Muscle biopsy showed mild dystrophic features associated to mild increase of lipid storage.

NGS multigene panel testing for hereditary myopathies identified the presence of a homozygous variant c.294G $>$ C (p.R98R) in POGLUT1 gene; healthy parents are heterozygous carriers of the same variant. A family with adult-onset LGMD has been recently described carrying a homozygous POGLUT1 p.R98W variant, which might indicate that an alteration in this amino acidic sequence could play a relevant role in protein functionality.

Our report extends the genotypic and phenotypic spectrum of myopathies associated to POGLUT1 gene. 
Creatine kinase and progression rate in Amyotrophic Lateral Sclerosis

\author{
M. Ceccanti ${ }^{1}$, C. Cambieri ${ }^{1}$, L. Libonati ${ }^{1}$, E. Onesti ${ }^{1}$, M. Inghilleri ${ }^{1}$. \\ ${ }^{1}$ Rare Neuromuscular Diseases Centre, Department of Human \\ Neurosciences, Sapienza University, Rome, Italy
}

Objectives: The purpose of this study was to evaluate CK in ALS patients over time, related to the disease progression, and differences with other neuropathies such as chronic inflammatory demyelinating polyradiculoneuropathy (CIDP).

Methods: In 126 fast or slow disease progressors ALS patients, CK levels were assayed for 16 months every 4 months. CK was also measured at baseline in 88 CIDP patients with secondary axonal damage and two mouse strains (129SvHSD and C57-BL) carrying the same SOD1G93A transgene expression but showing a fast (129Sv-SOD1G93A) and slow (C57- SOD1G93A) ALS progression rate.

Results: Higher CK was found in ALS slow progressors compared to fast progressors in $\mathrm{T} 1, \mathrm{~T} 2, \mathrm{~T} 3$ and $\mathrm{T} 4$, with a correlation with ALSFRS-R scores. Higher CK was found in spinal compared to bulbar-onset patients. Transgenic and non-transgenic C57BL mice showed higher CK levels compared to 129SvHSD strain. At baseline mean CK was higher in ALS compared to CIDP.

Conclusions: CK can predict the disease progression, with slow progressors associated to higher levels and fast progressors to lower levels, in both ALS patients and mice. CK is higher in ALS patients compared to patients with CIDP with secondary axonal damage; the higher levels of CK in slow progressors patients, but also in C57BL transgenic and non-transgenic mice designs $\mathrm{CK}$ as a predisposing factor for disease rate progression.

\section{Intravenous Onasemnogene Abeparvovec Clinical Development Programs in Spinal Muscular Atrophy (SMA): Integrated Safety Report}

D. Chand ${ }^{1}$, R.S. Finkel ${ }^{2}$, E. Mercuri ${ }^{3}$, R. Masson ${ }^{4}$, J. Parsons ${ }^{5}$, R. Cerbini ${ }^{1}$, F. Baldinetti $^{1}$, A. Kleyn ${ }^{1}$, M. Menier ${ }^{1}$, K. Montgomery ${ }^{1}$, D.M. Sproule ${ }^{1}$, S.P. Reyna $^{1}$, D.E. Feltner ${ }^{1}$, S. Tauscher-Wisniewski ${ }^{1}$, J.R. Mendell ${ }^{6,7,8}$

${ }^{1}$ AveXis, Inc., Bannockburn, IL, United States

${ }^{2}$ Division of Neurology, Department of Pediatrics, Nemours Children's Hospital, Orlando, FL, United States

${ }^{3}$ Department of Paediatric Neurology and Nemo Clinical Centre, Catholic University, Rome, Italy

${ }^{4}$ Developmental Neurology Unit, Fondazione IRCCS Istituto Neurologico Carlo Besta, Milan, Italy

${ }^{5}$ Department of Pediatrics, University of Colorado School of Medicine, Aurora, CO, United States

${ }^{6}$ Center for Gene Therapy, Nationwide Children's Hospital, Columbus, $\mathrm{OH}$, United States

${ }^{7}$ Department of Pediatrics, Ohio State University, Columbus, OH, United States

${ }^{8}$ Department of Neurology, Ohio State University, Columbus, $\mathrm{OH}$, United States

Intravenous (IV) onasemnogene abeparvovec (formerly AVXS-101) gene therapy addresses the genetic root cause of SMA, survival motor neuron 1 gene (SMN1) deletion/mutation. This report describes the safety of IV onasemnogene abeparvovec in SMA patients across 4 clinical trials. Symptomatic or presymptomatic patients (2-4XSMN2) received a single onasemnogene abeparvovec infusion. Adverse events (AEs) were assessed per CTCAE.
As of 8 March 2019, 75 patients received IV onasemnogene abeparvovec at the therapeutic dose $(1.1 \mathrm{e} 14 \mathrm{vg} / \mathrm{kg})$. Mean age at dosing: $2.5(0.3-7.9)$ months; mean weight: $5.33(3.0-8.4) \mathrm{kg}$. Two deaths were reported: 1 patient, aged 7.8 months, died of respiratory arrest 5.7 months postdosing (unrelated to treatment); 1 patient, aged 6.8 months, died of hypoxic/ischemic encephalopathy and an acute illness.

Sixty-four (85\%) patients reported $\geq 1$ AE; 33 (44\%) patients had $\geq 1$ treatment-related AE; 29 (39\%) patients had $\geq 1$ serious AE. Vomiting and pyrexia were reported as AEs at rates of $>5 \%$ in the clinical development program (considered treatment-related). Pyrexia was reported in $30(40 \%)$ patients ( 9 patients $\leq 1$ week after dosing) and was considered treatmentrelated in $4(5 \%)$ patients. Increased liver transaminases (>upper limit of normal) were observed in $8(11 \%)$ patients (considered treatment-related, clinically asymptomatic). Transient thrombocytopenia was reported, without clinically significant bleeding or bruising. There was no evidence of cardiac safety concerns associated with onasemnogene abeparvovec. Updated safety data (31 December 2019 datacut) will be presented.

The IV onasemnogene abeparvovec safety profile remains consistent with the United States package insert and continues to be monitored across multiple settings.

Open-label Study of Patisiran in Patients with hATTR Amyloidosis Post-Orthotopic Liver Transplant

T. Coelho ${ }^{1}$, J. Gillmore ${ }^{2}$, D. Adams ${ }^{3}$, F. Muñoz-Beamud ${ }^{4}$, A. Mazzeo ${ }^{5}, \mathrm{~J}$. Wixner $^{6}$, V. Planté-Bordeneuve ${ }^{7}$, L. Lladó ${ }^{8}$, S. Arum ${ }^{9}$, J.J. Wang ${ }^{9}$, X. $\mathrm{Li}^{9}$, H. Schmidt ${ }^{10}$

${ }^{I}$ Centro Hospitalar Universitário do Porto, Portugal;

${ }^{2}$ National Amyloidosis Centre, University College London, London, UK;

${ }^{3}$ National Reference Center for FAP (NNERF)/APHP/INSERM U 1195/ CHU Bicêtre, Le Kremlin Bicêtre, France,

${ }^{4}$ Juan Ramón Jiménez Hospital, Huelva, Spain;

${ }^{5}$ Unit of Neurology and Neuromuscular Diseases, Department of Clinical and Experimental Medicine, University of Messina, Messina, Italy;

${ }^{6}$ Department of Public Health and Clinical Medicine, Umea University, Umeå, Sweden,

${ }^{7}$ University Hospital Henri Mondor, Créteil, France;

${ }^{8}$ Hospital Universitari de Bellvitge, Barcelona, Spain;

${ }^{9}$ Alnylam Pharmaceuticals, Cambridge, MA, USA;

${ }^{10}$ University Hospital Münster, Münster, Germany

hATTR is a rapidly progressive, potentially fatal disease caused by a variant in the TTR gene. Methods and Results: Phase 3 open-label study (NCT0386280) to evaluate the safety, efficacy, and pharmacokinetics (PK) of patisiran in hATTR with disease progression post-OLT. 23 patients received patisiran $0.3 \mathrm{mg} / \mathrm{kg}$ intravenously once every 3 weeks for 12 months. Median age was 58.0 years, $13(56.5 \%)$ were males, and 15 (65.2\%) had V30M mutation. At baseline, 1 (4.3\%) patient had polyneuropathy disability (PND) score I, 9 (39.1\%) had PND II, and 13 (56.5\%) had PND IIIA/B. Five patients $(21.7 \%)$ had New York Heart Association (NYHA) classification I, 5 (21.7\%) had NYHA II, and none had NYHA III or IV at study baseline. After 6 months, the mean reduction from baseline in serum TTR levels was $89.2 \% .23$ patients (100\%) had experienced an $\mathrm{AE}$, the majority of AEs were mild or moderate. Patisiran reduced serum TTR levels by $>85 \%$ through 6 months of treatment in hATTR amyloidosis with disease progression post-OLT. The safety profile remains consistent with the Phase 3 APOLLO study.

Tubular Aggregate Myopathy caused by activating mutation in STIM1: a functional study in myoblasts and myotubes deriving from affected patients toward the identification of new therapeutic targets 
E. Conte ${ }^{1}$, G.M. Camerino ${ }^{1}$, A. Pannunzio ${ }^{1}$, M. Coluccia ${ }^{1}$, M. Mora ${ }^{2}$, L.

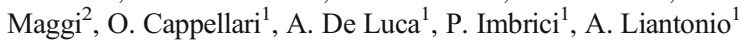

${ }^{1}$ Department of Pharmacy - Drug Sciences, University of Bari, Bari, Italy

${ }^{2}$ Neuromuscular Diseases and Neuroimmunology Unit, Foundation IRCCS Neurological Institute Carlo Besta, Milan, Italy

TAM is a hereditary ultra-rare progressive muscle disorder, clinically heterogeneous and involving proximal muscle weakness, muscle cramps or myasthenic features. Biopsies from TAM patients show the presence of tubular aggregates (TAs) originated from sarcoplasmic reticulum likely due to altered $\mathrm{Ca}^{2+}$ homeostasis. TAM is caused by gain-of-function mutations in STIM1 or ORAI1, proteins responsible for StoreOperated-Calcium-Entry (SOCE), a pivotal mechanism in $\mathrm{Ca}^{2+}$ signaling (Morin et al., Hum Mutation 2020). So far there is no cure for TAM and the mechanisms underlying disease in skeletal muscle of affected individuals remain to be clarified. It has been established that post-natal myogenesis critically relies on $\mathrm{Ca}^{2+}$ influx through SOCE. Thus, to explore how $\mathrm{Ca}^{2+}$ homeostasis dysregulation associated with TAM impacts on muscle proliferation and differentiation cascade, we here performed a functional characterization of myoblasts and myotubes deriving from patients carrying Leu96Val STIM1 mutant. We used a plethora of techniques raging from fura- 2 cytofluorimetry and high content imaging technology to real-time PCR. We demonstrated a significantly higher resting $\mathrm{Ca}^{2+}$ concentration and an increased SOCE in STIM1 mutant compared with control, together with an altered expression of RNAs coding for proteins regulating $\mathrm{Ca}^{2+}$ handling and muscle cell proliferation and differentiation. Importantly, a distinct pattern of multi-nucleated cells formation and different geometric features of mitochondrial network was detected in mutated myotubes, highlighting an altered fusion process associated to STIM1 mutation. Our functional study provide a correlation between dysregulation of $\mathrm{Ca}^{2+}$ homeostasis and myogenesis in TAM, toward the identification of novel druggable targets for this rare disease.

Ultra-high frequency ultrasound of median nerve. Comparison of different frequencies and correlation with clinical and neurophysiological findings

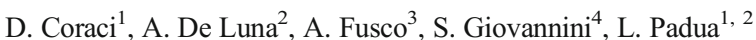

${ }^{1}$ Neuroriabilitazione ad Alta Intensità, Fondazione Policlinico Universitario A. Gemelli IRCCS, Rome, Italy

${ }^{2}$ Università Cattolica del Sacro Cuore, Milan, Italy

${ }^{3}$ IRCCS Fondazione Don Carlo Gnocchi, Milan, Italy

${ }^{4}$ Rehabilitation Units, Fondazione Policlinico Universitario A. Gemelli IRCCS, Rome, Italy

US efficacy for the evaluation of nerve structure is well-known. Recently, novel US machines, able to generate mechanical wave up to 50-70 MHz, have been produced. This ultra-high frequency US (UHF-US) allows an incredible increase of US resolution. In literature, a few studies present the application of UHF-US for the evaluation of peripheral nervous system. In these papers, the number of visible single fascicles is considerably higher than the routine US frequencies. In our study, we used a $50 \mathrm{MHz}$ UHF-US probe to evaluate median nerve at wrist, in healthy subjects. We assessed nerve cross sectional area and the number of the visible fascicles. The same US evaluation was performed using a $30 \mathrm{MHz}$ US probe. Furthermore, we assessed the sensory nerve conduction velocity and axonal potential amplitude of the median nerve, stimulating from the third digit. Subjects' age and body mass index (BMI) were recorded. We collected the data from twenty-two subjects. Number of visible fascicles were statistically different between the two frequencies. A difference was found for the measured nerve area, but with a perfect agreement. As expected, nerve area, independently from the frequency, was positively correlated with BMI $(\mathrm{p}<0.01)$, but only the number of fascicles visible with $50 \mathrm{MHz}$ were mildly correlated with BMI $(\mathrm{p}=0.03$ ). Finally, cross sectional area calculated by the UHF-US was positively correlate with nerve conduction velocity. This study, although preliminary, shows the potentialities of UHF-US. In particular, its elevated resolution and the possibility to reveal specific morphofunctional relationship should be considered.

\section{Biallelic mutations in sord cause a common and potentially treatable} neuropathy

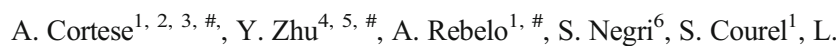
Abreu $^{1}$, C. J Bacon ${ }^{7}$, Y. Bai ${ }^{7}$, D. M Bis-Brewer ${ }^{1}$, E. Bugiardini ${ }^{2}$, E. Buglo $^{1}$, M. C Danzi ${ }^{1}$, S. ME Feely ${ }^{7}$, A. A Fragkouli ${ }^{2}$, N. A Haridy ${ }^{2}, 8$,

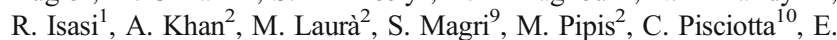
Powell $^{1}$, A. M Rossor ${ }^{2}$, J. Sowden ${ }^{11}$, S. Tozza ${ }^{12}$, J. Vandrovcova ${ }^{2}$, J. Dallman $^{13}$, E. Grignani ${ }^{6}$, E. Marchioni ${ }^{14}$, S. S Scherer ${ }^{15}$, B. Tang ${ }^{16}$, Z. Lin $^{17}$, A. Al-Ajmi ${ }^{18}$, R. Schüle ${ }^{19,20}$, M. Synofzik ${ }^{19,20}$, T. Maisonobe ${ }^{21}$, T. Stojkovic ${ }^{22}$, M. Auer-Grumbach ${ }^{23}$, M. A Abdelhamed ${ }^{8}$, S. A. Hamed $^{8}$, R. Zhang ${ }^{17}$, F. Manganelli ${ }^{12}$, L. Santoro ${ }^{12}$, P. Saveri ${ }^{9}$, F. Taroni $^{9}$, D. Pareyson ${ }^{10}$, H. Houlden ${ }^{2}$, D. N Herrmann ${ }^{11}$, M. M Reilly ${ }^{2}$, M. E Shy ${ }^{7}$, G. Zhai ${ }^{4}, 5$, S. Zuchner ${ }^{1}$

${ }^{I}$ Dr. John T. Macdonald Foundation Department of Human Genetics and John P. Hussman Institute for Human Genomics, University of Miami Miller School of Medicine, Miami, Florida, USA.

${ }^{2}$ Department of Neuromuscular Disease, UCL Queen Square Institute of Neurology and The National Hospital for Neurology, London, UK

${ }^{3}$ Department of Brain and Behavioral Sciences, University of Pavia, Pavia, Italy.

${ }^{4}$ Department of Molecular and Cellular Pharmacology, University of Miami Miller School of Medicine, Miami, Florida, USA

${ }_{5}^{5}$ Program in Molecular and Cellular Pharmacology, University of Miami Miller School of Medicine, Miami, Florida, USA

${ }^{6}$ Environmental Research Center, Istituiti Clinici Scientifici Maugeri IRCCS, Pavia, Italy

${ }^{7}$ Department of Neurology, University of Iowa Carver College of Medicine, Iowa City, Iowa, USA

${ }^{8}$ Department of Neurology and Psychiatry, Faculty of Medicine, Assiut University Hospital, Assiut, Egypt

${ }^{9}$ Unit of Medical Genetics and Neurogenetics, Fondazione IRCCS Istituto Neurologico Carlo Besta, Milan, Italy

${ }^{10}$ Unit of Rare Neurodegenerative and Neurometabolic Diseases, Department of Clinical Neurosciences, Fondazione IRCCS Istituto Neurologico Carlo Besta, Milan, Italy

${ }^{11}$ Department of Neurology, University of Rochester, Rochester, NY, USA

12 Department of Neuroscience, Reproductive Sciences and Odontostomatology, University of Naples "Federico II", Naples, Italy

${ }^{13}$ Department of Biology, University of Miami, Coral Gables, Florida, USA.

${ }^{14}$ IRCCS Mondino Foundation, Pavia, Italy

${ }^{15}$ Department of Neurology, Perelman School of Medicine, University of Pennsylvania, Philadelphia, Pennsylvania USA.

${ }^{16}$ National Clinical Research Center for Geriatric Disorders, Central South University, Changsha, 410078, Hunan Province, China

${ }_{17}$ Department of Neurology, The Third Xiangya Hospital, Central South University, Changsha, 410013, Hunan Province, China

${ }^{18}$ Division of Neurology, Department of Medicine, Al-Jahra Hospital, Kuwait

${ }^{19}$ Department of Neurodegenerative Disease, Hertie-Institute for Clinical Brain Research, and Center for Neurology, University of Tübingen, Tübingen, Germany 
${ }^{20}$ German Center of Neurodegenerative Diseases (DZNE), Tübingen, Germany

${ }^{21}$ AP-HP, G-H Pitié Salpêtrière, Department of Neurophysiology, Paris France.

${ }^{22}$ G-H Pitié-Salpêtrière, Centre de Référence des Maladies Neuromusculaires, Paris Nord/Est/Ile de France, Paris, France.

${ }^{23}$ Department of Orthopaedics and Traumatology, Medical University of Vienna, Vienna, Austria

\# Authors contributed equally

Introduction: As opposed to CMT1, for which over $90 \%$ of cases have mutations in known genes, only $20-30 \%$ of CMT2/dHMN patients receive a genetic diagnosis. Since up to $70 \%$ of CMT2 and $\mathrm{dHMN}$ cases are sporadic, identifying candidate pathogenic genes from single cases remains challenging also for nextgeneration sequencing techniques.

Methods: We took advantage of the largest collection of over 1,100 CMT patients in whom WES and/or WGS has been performed in the GENESIS analysis platform. We specifically looked for genes for which significant DNA variants are present in multiple families as well as for individual alleles overrepresented in CMT cases.

Results: We identified 45 cases from 38 families across multiple ethnicities, carrying a particular nonsense mutation in SORD, c.753delG;p.Ala253GlnfsTer27, SORD is an enzyme that converts sorbitol into fructose, in the two-step polyol pathway that has been implicated in diabetic neuropathy. In patients we find a complete loss of SORD function and 100x increased serum fasting sorbitol level. In Drosophila, we show that loss of SORD orthologues causes synaptic degeneration and progressive motor impairment. Reducing the polyol influx by treatment with aldose reductase inhibitors normalized intracellular sorbitol levels in patient fibroblasts and in Drosophila, and also dramatically ameliorated motor and eye phenotypes.

Conclusions: We demonstrate biallelic mutations in sorbitol dehydrogenase (SORD) as the most frequent recessive form of hereditary neuropathies. These findings establish a potentially treatable cause in a significant fraction of patients with inherited neuropathies and may contribute to a better understanding of the pathophysiology of diabetic neuropathy.

\section{Serum pattern of metalloproteinases- 2 and-9 and tissue inhibitors in} patients with chronic inflammatory demyelinating polyneuropathy

G. Cosentino ${ }^{1,2}$, V. Di Stefano ${ }^{3}$, M. Montana ${ }^{4}$, E. Alfonsi ${ }^{2}$, C. Tassorelli $^{1,2}$, F. Brighina ${ }^{3}$, B. Fierro ${ }^{3}$, G. Caimi ${ }^{4}$.

${ }^{1}$ Department of Brain and Behavioral Sciences, University of Pavia, Pavia, Italy

${ }^{2}$ IRCCS Mondino Foundation, Pavia, Italy

${ }^{3}$ Department of Biomedicine Neuroscience and Advanced Diagnostics, University of Palermo, Palermo, Italy

${ }^{4}$ Department of Health Promotion, Mother and Child Care, Internal Medicine and Medical Specialties, University of Palermo, Palermo, Italy.

Background: Metalloproteinases (MMPs) are a heterogeneous family of endopeptidases that play a role in many physiological functions, including the immune response. They are involved in remodeling of extracellular matrix and cell migration, and interact with cytokines and cell motility factors. ${ }^{1}$ An imbalance between activity of MMPs and their physiological tissue inhibitors (TIMPs) has been supposed to be involved in the pathophysiology of different autoimmune disorders. ${ }^{2}$

Aim: To assess the levels of MMP-2, MMP-9 and their inhibitors TIMP1 and -2 in patients with chronic inflammatory demyelinating polyneuropathy (CIDP).

Subjects and methods: Twenty patients with CIDP and 20 age- and sexmatched healthy controls were enrolled. Plasma concentrations of MMP-
2 and MMP-9 and their inhibitors TIMP-1 and TIMP-2 were determined by the enzyme-linked immunosorbent assay (ELISA).

Results: MMP-9 levels were higher and TIMP-1 lower in CIDP patients compared to controls, with significant increase in the MMP-9/TIMP-1 ratio. Conversely, levels of MMP-2 were found to be reduced in the patients' group, in the absence of changes in the TIMP-2 levels and MMP-2/TIMP-2 ratio.

Discussion and conclusions: A dysregulation of the serum pattern of MMPs and TIMPs, with upregulation of MMP-9 and downregulation of TIMP-1 and MMP-2 represents a useful biomarker of CIDP. Future studies are needed to evaluate whether the observed alterations are linked to the pathogenesis of CIDP or represent an epiphenomenon of the pathogenetic mechanisms underlying the peripheral nerve damage.

\section{References}

1) Morrison CJ, Butler GS, Rodríguez D, Overall CM. Matrix metalloproteinase proteomics: substrates, targets, and therapy. Curr Opin Cell Biol. 2009;21(5):645-53

2) Nissinen L, Kähäri V-M. Matrix metalloproteinases in inflammation. Biochim Biophys Acta - Gen Subj. 2014 Aug;1840(8):2571-80

Morphological analysis of TNPO3 and SRSF1 interaction during myogenesis: a super resolution study

R. Costa ${ }^{1,2}$, M.T. Rodia ${ }^{1,2}$, N. Zini ${ }^{3,4}$, V. Pegoraro ${ }^{5}$, R. Marozzo ${ }^{5}$, C. Capanni $^{3,4}$, C. Angelini ${ }^{5}$, G. Lattanzi ${ }^{3},{ }^{4}$, S. Santi ${ }^{3,4}{ }^{4}$, G. Cenacchi ${ }^{1,2}$.

${ }^{I}$ Department of Biomedical and Neuromotor Sciences - DIBINEM, University of Bologna, Bologna, Italy.

${ }^{2}$ Center of Applied Biomedical Research-CRBA, University of Bologna, St. Orsola Hospital, Bologna, Italy.

${ }^{3}$ CNR - National Research Council of Italy, Institute of Molecular Genetics "Luigi Luca Cavalli-Sforza”, Unit of Bologna, Bologna, Italy.

${ }^{4}$ IRCCS Istituto Ortopedico Rizzoli, Bologna, Italy.

${ }^{5}$ Neuromuscular Unit, Neurobiology Research group, IRCCS San Camillo Hospital, Venice, Italy.

Limb girdle muscular dystrophy D2 (previously LGMD1F) is a rare neuromuscular disease due to a heterozygous mutation in the TNPO3 gene coding for Transportin 3 (TNPO3). TNPO3 is an importin that shuttles, from the cytoplasm to the nucleus, the serine/arginine-rich proteins (SRproteins) among which the splicing factor SRSF1. The SR family includes proteins involved in mRNA metabolism and essential splicing factors that, regulating alternative splicing, contribute to post transcriptional gene regulation. In this way they affect the proteomic diversity in muscle, contributing to the control of satellite cell fate during muscle differentiation and helping the maintenance of healthy muscle. Since the strict interaction between SRSF1 and TNPO3, a mutation in TNPO3 could downstream dysregulate SRSF1 function, affecting splicing and causing abnormalities in fibers that contribute to LGMD D2 pathogenesis.

We analyzed if myogenic differentiation could be modulated by interactions between SRSF1 and TNPO3. The different steps of myogenesis have been monitored in $\mathrm{C} 2 \mathrm{C} 12$ cells and TNPO3 expression have been investigated by confocal and electron microscopy, showing that it increases in nuclei of differentiated myotubes. The interactions of TNPO3 and SRSF1 have been quantitatively evaluated by a structured illumination microscope: SRSF1 remains localized in the nucleus, while TNPO3 decreases in the cytoplasm appearing strongly clustered in the nucleus of myotubes, suggesting that it could act in the proteomic network necessary during myogenesis. These data stress the role of TNPO3 as a carrier of SRSF1 in crucial steps of myogenic differentiation and could help to clarify the pathogenic mechanisms of LGMD D2. 


\section{Brown-Vialetto-Van Laere Syndrome: a clinical report}

S. Cotti Piccinelli ${ }^{1}$, F. Novara ${ }^{2}$, B. Risi ${ }^{1}$, E. Baldelli ${ }^{1}$, N. Necchini ${ }^{1}$, A. Galvagni $^{1}$, R. Ciccone ${ }^{2}$, A. Padovani ${ }^{1}$, M. Filosto

${ }^{1}$ Center for Neuromuscular Diseases, Unit of Neurology, ASST Spedali Civili and University of Brescia, Brescia, Italy

${ }^{2}$ Microgenomics Laboratory, Pavia, Italy

Brown-Vialetto-Van Laere Syndrome (BVS) is an extremely rare disorder with fewer than 100 cases reported in the literature, mainly characterized by sensorineural deafness, lower cranial nerve and lower motor neuron involvement, impaired respiratory function, and, in a variable way, optic atrophy, pigmentary retinitis, autonomic dysfunction and epilepsy.

The disease is caused by mutations in the SLC52A2 and SLC52A3 genes encoding for the human riboflavin transporters RFVT2 and RFVT3.

Several studies have reported improvement in clinical outcomes with riboflavin supplementation.

We report on a 42 year-old man suffering from severe hearing loss, chronic respiratory failure and optic atrophy since childhood.

Neurological examination showed weakness and hypotrophy of the intrinsic muscles of the hands, ataxic gait, mild cerebellar signs in the limbs, absence of ankle jerk reflexes, severe hearing loss and visual acuity reduction with erratic eye movements.

Chronic neurogenic signs were detected on EMG and in muscle biopsy. CK levels were within normal range.

Genetic analysis by clinical exome revealed two novel heterozygote variants (c.353C > A; p.Ala118Asp and c.863C > T; p.Ala288Val) in the SLC52A2 gene.

Oral riboflavin $10 \mathrm{mg} / \mathrm{kg} /$ day was started which was further increased to $20 \mathrm{mg} / \mathrm{kg} /$ day after a week and patient was followed-up.

BVS is difficult to diagnose because its rarity. Nevertheless, an early diagnosis must be actively sought because riboflavin may be an effective therapy, especially in younger patients, or, at least, shift the clinical course.

An early therapeutic attempt with riboflavin appears to be crucial as diaphragmatic paralysis may be irreversible.

\section{Expanding the phenotype of p.r1460w mutation in scn4a gene: a family report}

S. Cotti Piccinelli ${ }^{1}$, B. Risi ${ }^{1}$, E. Baldelli ${ }^{1}$, N. Necchini ${ }^{1}$, A. Galvagni ${ }^{1}$, A. Padovani $^{1}$, R. Brugnoni ${ }^{2}$, L. Maggi ${ }^{2}$, M. Filosto ${ }^{1}$

${ }^{1}$ Center for Neuromuscular Diseases, Unit of Neurology, ASST Spedali Civili and University of Brescia, Brescia, Italy

${ }^{2}$ Foundation IRCCS Neurological Institute Carlo Besta, Milan, Italy

$S C N 4 A$ gene encodes the pore-forming $\alpha$ subunit of the voltage-gated sodium channel $\left(\mathrm{Na}_{\mathrm{V}} 1.4\right)$. Gain-of -function mutations are usually associated with myotonia, paramyotonia, or Periodic Hyper-Hypokalemic Paralysis and are all dominantly inherited. Loss-of-function mutations are less frequent, are inherited in a recessive manner and are associated with congenital myasthenic phenotypes.

The homozygote loss-of-function p. R1460W mutation has been described in a patient affected with myasthenic congenital myopathy. Both heterozygote parents were asymptomatic, suggesting a recessive transmission.

We report a family (two brothers, one sister and her son) complaining about fatigue, muscle cramps, pain and stiffness after physical activity since adolescence. The brothers experienced episodes of periodic paralysis improved after potassium administration.
EMG study showed myopathic abnormalities in the younger brother and was normal in the other subjects. Muscle biopsy performed on the younger brother showed fiber diameter variability and scattered atrophic fibers. Genetic analysis has detected the heterozygote $\mathrm{p} . \mathrm{R} 1460 \mathrm{~W} S C N 4 A$ variant in all the siblings. Their asymptomatic mother showed no mutation, while the father was not tested because he died early.

To our knowledge, this is the second family harboring p.R1460W in $S C N 4 A$ reported in literature. However, in our family, this mutation appears to be associated with an autosomal dominant "hypoPP, muscle pain and cramps" phenotype while myasthenic features were absent.

Our findings expand the phenotypical spectrum associated with the p.R1460W mutation in SCN4A, by reporting a novel phenotype and a novel inheritance pattern. Further investigation on possible epigenetic factors are mandatory in order to better define and understand the mechanisms underlying this variable clinical expression.

Guillain-Barré Syndrome and COVID-19: an observational multicenter study from Lombardy and Veneto (Italy)

S. Cotti Piccinelli ${ }^{1}$, S. Gazzina ${ }^{2}$, C. Foresti ${ }^{3}$, B. Frigeni ${ }^{3}$, C. Servalli ${ }^{3}$, M. Sessa $^{3}$, G. Cosentino ${ }^{4}$, E. Marchioni ${ }^{5}$, S. Ravaglia ${ }^{4}$, C. Briani ${ }^{6}$, F. Castellani $^{6}$, G. Zara $^{6}$, F. Bianchi ${ }^{7}$, U. Del Carro ${ }^{7}$, R. Fazio ${ }^{7}$, M. Filippi $^{7}$, E. Magni ${ }^{8}$, G. Natalini ${ }^{9}$, F. Palmerini ${ }^{8}$, A. M. Perotti ${ }^{8}$, A. Bellomo $^{10}$, M. Osio ${ }^{11}$, G. Scopelliti ${ }^{10}$, M. Carpo ${ }^{12}$, A. Rasera ${ }^{13}$, G. Squintani ${ }^{13}$, P. E. Doneddu ${ }^{14}$, V. Bertasi ${ }^{15}$ M.S. Cotelli ${ }^{15}$, G.M. Fabrizi $^{16}$, S. Ferrari ${ }^{16}$, L. Bertolasi ${ }^{16}$, F. Ranieri ${ }^{16}$, F. Caprioli ${ }^{17}$, E. Grappa $^{18}$, L. Broglio ${ }^{2}$, G. De Maria ${ }^{2}$, U. Leggio ${ }^{2}$, L. Poli ${ }^{1}$, F. Rasulo ${ }^{19}$, N. Latronico ${ }^{19}$, E. Nobile-Orazio ${ }^{14}$, A. Padovani ${ }^{1}$, A. Uncini ${ }^{20}, \mathrm{M}$. Filosto $^{21}$

${ }^{1}$ Department of Clinical and Experimental Sciences, University of Brescia; Unit of Neurology, ASST Spedali Civili, Brescia, Italy

${ }^{2}$ Unit of Neurophysiopathology, ASST Spedali Civili, Brescia, Italy

${ }^{3}$ Unit of Neurology and Neurophysiology, ASST PG23, Bergamo, Italy

${ }^{4}$ IRCCS Mondino Foundation, Department of Brain and Behavioral Sciences, University of Pavia, Italy

${ }^{5}$ IRCCS Mondino Foundation, Neurooncology and Neuroinflammation Unit, Pavia, Italy

${ }^{6}$ Neurology Unit, Azienda Ospedale-Università di Padova, Italy

${ }^{7}$ Neurology and Neurophysiology Unit, IRCCS San Raffaele Scientific Institute, Vita Salute San Raffaele University, Milano, Italy

${ }^{8}$ Unit of Neurology, Fondazione Poliambulanza, Brescia, Italy

${ }^{9}$ Unit of Intensive Care and Anesthesiology, Fondazione Poliambulanza, Brescia, Italy

10 "Luigi Sacco" Department of Biomedical and Clinical Sciences, University of Milan, Milano, Italy

${ }^{11}$ Unit of Neurology, ASST Fatebenefratelli Sacco, Milano

${ }^{12}$ Unit of Neurology, ASST Bergamo Ovest, Treviglio, Italy

${ }^{13}$ Unit of Neurology, AOUI Verona, Verona, Italy

${ }^{14}$ Department of Neurology, Neuromuscular and Neuroimmunology Service, IRCCS Humanitas Clinical and Research Institute; Department of Medical Biotechnology and Translational Medicine, Milan University, Milan, Italy

${ }^{15}$ Unit of Neurology, ASST Valcamonica, Esine (Brescia), Italy

${ }^{16}$ Neurology Unit, Department of Neuroscience, Biomedicine and Movement Sciences, University of Verona, Verona, Italy

${ }^{17}$ Unit of Neurology, ASST Cremona, Cremona, Italy

${ }^{18}$ Intensive Care Unit, ASST Cremona, Cremona, Italy

${ }^{19}$ Department of Anesthesia, Critical Care and Emergency, ASST Spedali Civili, Brescia; Department of Medical and Surgical Specialties, Radiological Sciences and Public Health, University of Brescia, Brescia, Italy 
${ }^{20}$ Department of Neuroscience, Imaging and Clinical Sciences, University "G. d'Annunzio", Chieti-Pescara, Italy

${ }^{21}$ Department of Clinical and Experimental Sciences, University of Brescia; NeMO-Brescia Clinical Center for Neuromuscular Diseases, Brescia, Italy

Background: Single cases and small series of Guillain-Barré syndrome (GBS) were reported during the SARS-CoV-2 outbreak . No univocal findings on incidence and clinical aspects of COVID-19-related GBS are available.

Methods: GBS cases diagnosed in 12 referral hospitals from Lombardy and Veneto in March and April 2020 were retrospectively investigated. As a control population, GBS patients diagnosed in March and April of 2019 in the same hospitals were considered.

Results: A total of 34 patients (30 COVID-positive GBS and 4 COVIDnegative GBS) were collected in 2020 while 13 control patients were diagnosed in 2019

Incidence of GBS in March and April 2020 was 0.202/100.000/ month (estimated rate 2.43/100.000/year) versus $0.077 / 100.000 /$ month (estimated rate $0.93 / 100.000$ /year) in the same months of 2019 with a 2.6-fold increase. Estimated incidence of GBS in COVID-19 positive patients was $47.9 / 100.000$ and in the COVID19 positive hospitalized patients was 236/100.000. When compared with COVID-19 negative patients, COVID-19 positive GBS subjects showed lower MRC sum score, higher frequency of demyelinating GBS subtype, more frequent low blood pressure and higher rate of admission to intensive care unit (ICU).

Conclusions: This study shows an increased incidence of GBS during COVID-19 outbreak in northern Italy compared to 2019, supporting a pathogenic link. COVID-19-associated GBS is predominantly demyelinating and is associated with a more severe motor impairment than non-COVID-19 GBS although it is likely that in some patients the systemic and pulmonary impairment due to COVID-19 might have contributed to the severity of the whole clinical picture.

\section{A genotyping and clinical neurophysiological study of early-onset Charcot-Marie-Tooth disease}

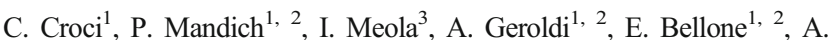
Accogli ${ }^{1}$, C. Bruno ${ }^{4}$, M. Pedemonte ${ }^{4}$, C. Minetti ${ }^{1}$, C. Fiorillo ${ }^{1}$, P. Lanteri ${ }^{5}$

${ }^{1}$ Department of Neurosciences, Rehabilitation, Ophthalmology, Genetics, Maternal and Child Health (DINOGMI), University of Genoa, Genoa, Italy;

${ }^{2}$ IRCCS Policlinico San Martino, Genoa, Italy

3 Unit of Child Neuropsychiatry, Child Hospital "Cesare Arrigo", Alessandria, Italy

${ }^{4}$ Center of Translational and Experimental Myology, IRCCS Istituto Giannina Gaslini, Genova, Italy.

${ }^{5}$ Neurophysiopathology Centre, Department of Diagnostics and Applied Technology, Fondazione IRCCS, Istituto Neurologico "C. Besta", Milan, Italy

Objective: To study the clinical and neurophysiological characteristics and genetic variation of a cohort of patients with early-onset CharcotMarie-Tooth disease (CMT) (before 2 years-old).

Methods: we report a 14 year (2003-2017) retrospective study of earlyonset CMT disease cases in IRCCS G Gaslini, Genoa. Medical history was recollected from the patients' files.

Results: A total of 44 cases were included in our analysis, with $39 / 44$ genetic confirmation $(88.6 \%)$ with 11 different genes involved. The male to female ratio was 1.1:1. Seventeen patient have global psychomotor delay, in particular 12 patients with no perinatal sufferance. $29.5 \%$ (13 patients) had sporadic mutation in various genes, two patients were foster. $66 \%$ of patients had demyelinating CMT, $18.1 \%$ had axonal CMT and $11.3 \%$ had a normal conduction velocities study.

The genetic test results of these children showed 8 cases of $17 \mathrm{p} 11$ duplication and 3 deletions, 5 cases of GDAP mutation, 5 cases of GJB1 mutation, 4 cases of MPZ gene insertion mutation, 4 of PMP22, 2 cases of MFN2 gene point mutation, 2 cases with SH3TC2 mutation, 2 TPRV4, 2 of NDRG1, 1 GARS. Leading causes of axonal CMT were GDAP (5/8), MFN2 (1/8), TRPV4 (1/8).

Conclusions: early-onset CMT cases were most likely to receive a molecular diagnosis that encompasses a wide variety of inherited sensory and/or motor neuropathies.

Biallelic RFC1 expansion is a common cause of idiopathic sensory neuropathy and ganglionopathy

R. Currò ${ }^{1,2}$, A. Salvalaggio ${ }^{3}$, S. Tozza ${ }^{4}$, C. Gemelli ${ }^{5}$, N. Dominik ${ }^{6}$, V. Galassi Deforie $^{6}$, F. Castellani ${ }^{3}$, E. Vegezzi ${ }^{1,2}$, S. Efthymiou ${ }^{6}$, G. Cosentino $^{1,2}$, E. Alfonsi ${ }^{2}$, E. Marchioni ${ }^{2}$, S. Colnaghi ${ }^{2}$, EM. Valente ${ }^{7}$,

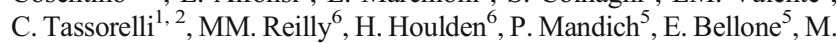
Grandis $^{5}$, A. Schenone ${ }^{5}$, L. Santoro ${ }^{4}$, F. Manganelli ${ }^{4}$, C. Briani ${ }^{3}$, A. Cortese $^{1,6}$

${ }^{1}$ Department of Brain and Behavioral Sciences, University of Pavia, Pavia, Italy

${ }^{2}$ IRCCS Mondino Foundation, Pavia, Italy

${ }^{3}$ Department of Neurosciences, University of Padova, Padova, Italy

${ }^{4}$ Department of Neuroscience, University of Naples, Naples, Italy

${ }^{5}$ DINOGMI, University of Genoa, Genoa, Italy; Neurology Unit, IRCCS San Martino Hospital, Genoa, Italy, Genoa, Italy,

${ }^{6}$ Department of Neuromuscular Diseases, University College London, London, United Kingdom

${ }^{7}$ Department of Molecular Medicine, Unit of Genetics, University of Pavia, Pavia, Italy

Introduction: The differential diagnosis of chronic sensory neuropathies (SN) and sensory ganglionopathy (SG) includes metabolic, toxic, inflammatory and few genetic causes. However, a large fraction of patients remains undiagnosed. Recently, biallelic repeat expansion in the RFC1 gene has been recognised as the cause of CANVAS. To date, all genetically confirmed CANVAS patients have a sensory neuropathy. Hence, we investigated the prevalence of biallelic RFC1 expansion in patients with idiopathic SN or SG. Methods: Patients diagnosed with SN or SG were retrospectively identified through revision of electrodiagnostic studies. Cases with known causes or previously diagnosed as CANVAS were excluded. Patients with idiopathic SN or SG were clinically re-evaluated and a blood sample was collected for RFC1 testing.

Results: Eighty-three patients were enrolled. Biallelic RFC1 expansion was identified in 29 cases (36\%), and in particular in 21 patients with SN and in 8 patients with SG. Most common complaints at onset were unsteadiness $(72 \%)$, tingling and pin-and-needles (48\%) and numbness (38\%). Chronic cough, pain and dysautonomia were present in $50 \%$, $41 \%$ and $24 \%$ of patients, respectively. Examination showed abnormal vibratory and pinprick sensation (72\% and 58\%), whilst joint position was preserved in almost all cases (7\%). 50\% had nystagmus. Vestibuloocular reflex (VOR) was tested in 18 patients and was abnormal in $39 \%$ out of them.

Conclusions: This study suggests that biallelic RFC1 expansion accounts for a high percentage of idiopathic SN and SG. RFC1 testing should be considered in all these cases, particularly in presence of chronic cough or subclinical cerebellar and/or vestibular involvement. 
CMV primary infection and GBS, a treatment open question. Report of two cases and review

S. Dallagiacoma ${ }^{1}$, S. Bocci ${ }^{1}$, S. Bartalini ${ }^{1}$, S. Ferrone ${ }^{1}$, L. Franci ${ }^{1}$, F. Ginanneschi ${ }^{1}$, F. Giannini ${ }^{1}$

${ }^{1}$ Neurology and Clinical Neurophysiology Unit, Department of Medicine, Surgery and Neurosciences, University of Siena, Italy

CMV is the most common antecedent viral infection in adult GBS patients which often show AIDP type, are younger, develop facial nerve palsy and have sensory loss and severe course. Case 1. A 37-year-old male with five days distal paraesthesia and impaired balance one week after febrile illness showed gloves/socks hypoesthesia, unstable gait and limb areflexia. Case 2. A 51-year-old female with one day tingling/ numbness in hands and legs and diffuse fatigability two weeks after febrile illness showed lower limbs hyporeflexia. In both patients lab tests revealed CSF cytoalbuminological dissociation, serum positive antiCMV-IgM, low avidity anti-CMV-IgG, CMV-DNA, negative antigangliosides antibodies and MRI cauda roots enhancement. Neurophysiological study showed features of AIDP (day-20/case-1; day-23/case-2) with acute axonal degeneration (case-2). Ganciclovir 5 $\mathrm{mg} / \mathrm{Kg} /$ die i.v. until negative DNA was administered. Case-1 received five sessions of PE nonetheless he developed asymmetrical facial palsy and symmetric proximal weakness $(3 / 5)$ on day 14 (GBSDS=3). IVIg $(2$ $\mathrm{gr} / \mathrm{kg}$ ) were infused and showed GBSD $=1$ at discharge (day 26).Case-2 received IVIg (2gr/day) but developed (day 8) left facial palsy, dysphagia, symmetric severe weakness, areflexia, autonomic and respiratory failure requiring NIV (GBSDS=5). GBSDS was 1 at three months follow-up. The pathogenic mechanism of CMV infection in the GBS is yet unclear. It can be hypothesized that primary CMV infection $(12.5 \%$ of a French GBS cohort) may directly invade PNS contributing to inflammation, beyond the trigger of autoimmune response. Detecting IgG-avidity is essential to diagnose this condition. Only a properly designed trial could answer whether additional early antiviral therapy is recommended.

\section{Discordant clinical outcome in two cousins with $\mathrm{X}$-linked myotubular myopathy}

P. D’Ambrosio ${ }^{1}$, M.G. Esposito ${ }^{2}$, S. Morra $^{1}$, A. Palladino ${ }^{1}$, L.

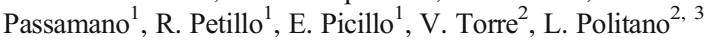

${ }^{1}$ U.O.i.D. di Genetica Medica e Cardiomiologia - AOU dell'Università della Campania Luigi Vanvitelli - Napoli, Italy

${ }^{2}$ Unità di Ricerca Centro Gaetano Torre per Le Malattie MuscolariNapoli, Italy

${ }^{3}$ Centro Clinico NeMO "Giovanni Nigro" - Napoli, Italy

X-linked myotubular myopathy (XLMTM) is a life-threatening, congenital myopathy characterized by extreme hypotonia, weakness, delayed motor milestones, and respiratory failure, often resulting in pediatric mortality. It is caused by pathogenic variants in the myotubularin 1 (MTM1) gene.

We report 2 male patients, belonging to the same family, first cousins - the mothers are sisters - and the third is the son of a female cousin of the mothers. The first patient - LP - came to our observation due to gait disturbances and $\mathrm{CK}$ values slightly elevated, at the age of 12 years. The physical examination showed severe thinness, elongated facies, pointed palate and protruding ears, characteristic of a congenital myopathy. At the current age of 31 years and 3 months, the patient is still able to walk independently; NSAA is $23 / 34$; Gowers' time is $3.25 \mathrm{sec}$; run time is 2.56 seconds. FVC is $2250 \mathrm{ml}(44 \%$ of the theoretical value). No assisted ventilation. No cardiomyopathy.

The cousin - PD - came to our observation at the age of 6 years and 7 months for gait disturbances and family history. Birth at term with perinatal asphyxia. Walking at 18 months with a staggering gait and internal rotation of feet. Recurrent episodes of bronchopneumonia. LoA at the age of 10. Normal CK values. Non-invasive mechanical ventilation started at 6 years and 6 months. At the current age of 34 years and 5 months, the patient has a severe kyphoscoliosis. FVC is $580 \mathrm{ml}(15 \%$ of the theoretical value). No cardiomyopathy.

Phenotypic and genetic characterization of childhood CharcotMarie-Tooth disease

FR. Danti ${ }^{1}$, S. Magri ${ }^{2}$, S.Genitrini ${ }^{1}$, E. Pagliano ${ }^{1}$, M. Foscan ${ }^{1,}$ A. Ardissone ${ }^{1}$, C. Ciano ${ }^{3}$, P. Saveri ${ }^{4}$, F.Balistreri ${ }^{2}$, D. Di Bella ${ }^{2}$, D. Pareyson $^{4}$, F. Taroni ${ }^{2}$, I. Moroni ${ }^{1}$.

${ }^{1}$ Department of Pediatric Neurosciences;

${ }^{2}$ Genetics of Neurodegenerative and Metabolic Diseases Unit;

${ }^{3}$ Neurophysiopatology Unit;

${ }^{4}$ Department of Clinical Neurosciences, Fondazione IRCCS Istituto Neurologico Carlo Besta, Milan

Charcot-Marie-Tooth disease (CMT), the commonest inherited neuromuscular disorder, is characterized by a wide genetic and phenotypic heterogeneity and variable age of onset from infancy to adulthood; however only few reports focusing on large pediatric cohorts have been described so far.

We presented 300 pediatric patients with CMT disease referred to our Institute over a 20 -year period with the aim of characterizing in detail the clinical, electrophysiological and genetic spectrum. The demyelinating form was the most frequent subtype (CMT1) $(63 \%)$, followed by the axonal or intermediate ones (24\%); pure motor (HMN) and sensory (HSN) forms accounted for about $10 \%$ of patients.

A definite diagnosis was achieved in $80 \%$ of patients with CMT1, in $60 \%$ with CMT2, in $30 \%$ with HMN, by Sanger sequencing and/or target NGS gene panels. CMT1A represents the most frequent subtype (46\% of total, $74 \%$ of demyelinating forms). The other genetic defects were largely more rare, with mutations in $M P Z, M F N 2, G D A P 1$ and $G J B 1$ genes accounting for $5 \%$ of patients each, and mutations in other genes detected in few or single cases.

Median age of onset was 7 years in patients with CMT1A. In the other subtypes, the age of first symptoms was less than 6 years in $70 \%$ and less than 2 years in $30 \%$ of patients.

Peculiar clinical features provided useful clues to address targeted genetic testing, such as very early onset (PMP22, MPZ, EGR2), cranial nerve involvement (GDAP1, TRIM2), mental retardation (MCM3AP), neuromyotonia (HINTI), early onset scoliosis, (IGHMBP2, SH3TC2).

\section{Atypical Acute Motor-Sensitive Axonal Neuropathy (AMSAN) dur- ing Acute Hepatitis B Virus (HBV) infection}

N. De Angelis ${ }^{1}$, P. Galluzzi ${ }^{2}$, F. Parodi ${ }^{1}$, S. Bocci $^{1}$, F. Ginanneschi ${ }^{1}$, C. Battisti $^{1}$, F. Giannini ${ }^{1}$

${ }^{1}$ Dipartimento di Scienze mediche, chirurgiche e neuroscienze, Università degli studi di Siena, Italy

${ }^{2}$ Dipartimento di Scienze mediche, UOC Malattie infettive e tropicali, Azienda Ospedaliera Universitaria Senese, Italy

Guillain-Barré Syndrome (GBS) have been rarely reported in association with acute HBV infection. We present a case of a 79-year-old man, who was admitted to neurology department with a 2-week history of distal paresthesias and tactile hypoaesthesia in lower extremities and right arm. One month before he had presented fever, asthenia and weight loss, with mild increase of hepatic enzymes. His neurological exam showed hypo/areflexia in lower limbs, and a progressive and severe motor impairment in distal limbs and proximal right leg, that reached acme in 5 weeks (Rankin Score 4). Electrophysiological study showed Reversible 
Conduction Failure in right ulnar nerve and was suggestive of AMSAN. Lab investigations demonstrated HBV-DNA quantitative PCR $>1,000,000,000 \mathrm{UI} / \mathrm{ml}$, positive $\mathrm{HBs} \mathrm{Ag}$ and anti-HBc IgM, and negative anti-HBs and anti-HBe $\mathrm{Ab}$. He was treated with intravenous immunoglobulin and Entecavir. Clinical improvement occurred after 3 months along with reduction of viral load. Six-months follow-up showed quite full recovery of motor and sensitive impairment. How HBV can cause GBS remains unknown. Several mechanisms have been proposed, such as molecular mimicry between HBV-DNA and myelin basic protein, direct damage to the myelin by HBV, or HBsAg-mediated immune-complex vasculitis. Less than 20 cases of GBS associated with HBV infection are reported in the literature until today, all with demyelinating features although not properly detailed. The current patient presents atypical clinical course with delayed disease acme, prominent axonal features at electrophysiological study, and asymmetric pattern also compatible with vasculitic pathogenic component.

Unravelling combined RNA interference and gene therapy in vitro and in vivo disease models as a potential therapeutic strategy for CMT2A

R. De Gioia ${ }^{2}$, M. Nizzardo ${ }^{1,2}$, S. Bono ${ }^{2}$, S. Salani ${ }^{2}$, V. Melzi ${ }^{2}$, S. Pagliarani $^{2}$, E. Abati ${ }^{1}$, N. Bresolin ${ }^{1,2}$, G. Comi ${ }^{1,2}$, S. Corti ${ }^{1,2}$, F. Rizzo $^{1,2}$

\section{${ }^{1}$ University of Milan \\ ${ }^{2}$ IRCCS Foundation Ca' Granda Ospedale Maggiore Policlinico}

Charcot-Marie-Tooth type 2A (CMT2A) is an inherited, debilitating, sensory-motor neuropathy caused by missense mutations in the MFN2 (Mitofusin2) gene. MFN2 mutations appear to induce the disease with a dominant-negative mechanism, where the expression of the wild-type MFN2 allele is negatively regulated by the mutant protein. Gene therapy for dominantly inherited diseases with RNA interfering (RNAi) is to selectively inhibit the expression of the mutant allele, which produces a toxic protein. Because this treatment can also excessively reduce the expression of the wild-type allele, wildtype allele restoration, combined with mutant allele silencing, could increase the therapeutic effects. Here, we propose for the first time this combined strategy as possible CMT2A therapeutic approach. In particular, we demonstrated the effective silence of the endogenous MFN2 (both mutant and wild-type MFN2 alleles) and the its replacement with an exogenous copy of the wild-type MFN2 gene in CMT2A human induced pluripotent stem cells (iPSCs)-differentiated motor neurons (Rizzo et al 2016) and in Mitocharc1, a mouse model of CMT2A (Cartoni et al., 2010). To evaluate the amelioration of the disease phenotype after this strategy, we will analyze key motoneuronal features relevant to CMT2A, observing an enhancement in mitochondrial distribution and function, beyond in apoptotic and autophagic parameters. Our data confirm the feasibility of combined RNAi and gene therapy approach as potential therapeutic strategy for treating CMT2A and other genetic neurological disorders.

Therefore, we will test mutant MFN2 silencing with and without wild-type MFN2 restoration in in vitro and in vivo disease models, identifying the optimal strategy for translation to clinical trials.

\section{Myotonic dystrophy type 1: daytime pulmonary function and sleep related breathing disorders}

S. de Pasqua ${ }^{1}$, F. Pizza ${ }^{1,2}$, P. Avoni ${ }^{1,2}$, C. Quarta $^{3}$, G. Plazzi ${ }^{1,2}$, R. Liguori ${ }^{1,2}$

${ }^{1}$ DIBINEM, Department of Biomedical and Neuromotor Sciences, Alma Mater Studiorum, University of Bologna, Italy
${ }^{2}$ IRCCS Institute of Neurological Sciences of Bologna, Italy

${ }^{3}$ UO Pneumologia, Dipartimento Medico, AUSL Bologna, Italy

Background: Myotonic dystrophy type 1 (DM1) represents the neuromuscular disease with the most prominent sleep disorders, including excessive daytime sleepiness (EDS) and sleep related breathing disorders (SDB). SDB may be explained either by involvement of respiratory muscles or abnormalities of central control of ventilation. Previous studies failed to identify predictive clinical parameters of SDB.

Objectives: to evaluate sleep disorders in DM1, the correlation between nocturnal and daytime pulmonary function and the genotype-phenotype correlation.

Methods: 32 DM1 patients (7 E1, 20 E2 and 5 E3) performed 4 channel nocturnal cardiorespiratory monitoring, $10 / 32$ performed complete 8 channel monitoring. Daytime pulmonary function was studied through sprirometry and blood gas analysis.

Results: SDB was detected in 3/7 E1, 16/20 E2 and 5/5 E3 patients. Central apneas were present in $7 / 10$ patients. There was no correlation between class of expansion and severity of SDB. We observed a decreasing trend in forced vital capacity (CVF) and FEV1 with the expansion increase, while no correlation was found between class of expansion and blood gas analysis values. We found correlations between daytime $\mathrm{SpO} 2$ and ODI index, daytime $\mathrm{CO} 2$ and minimal nocturnal SpO2, FEV1 and average $\mathrm{SpO} 2$ during sleep.

Discussion: our results suggest that some daytime respiratory parameters could predict SDB. We found a high percentage of central apnea, confirming literature data about a possible CNS involvement in DM1 patients. The lack of significant correlations between class of expansion and blood gas analysis suggests the importance of periodic clinical follow-up including pulmonary tests to predict SDB.

Muscle manifestations and CK levels in COVID infection: results of a large cohort of patients inside a Pandemic COVID-19 Area

A.De Rosa ${ }^{1,2}$, E. Pinuccia Verrengia ${ }^{1}$, I.Merlo $^{3,4}$, F. Rea ${ }^{3,4}$, G. Siciliano $^{2}$, G. Corrao ${ }^{3,4}$, A. Prelle ${ }^{1}$

${ }^{1}$ UO Neurologia-Stroke Unit, AO Ospedale Civile di Legnano, ASST Ovest Milanese, Legnano, Italy.

${ }^{2}$ Neurology Unit, Department of Clinical and Experimental Medicine University of Pisa, Pisa, Italy

${ }^{3}$ National Centre for Healthcare Research \& Pharmacoepidemiology, University of Milano-Bicocca Milan, Italy

${ }^{4}$ Laboratory of Healthcare Research \& Pharmacoepidemiology, Unit of Biostatistics, Epidemiology and Public Health, Department of Statistics and Quantitative Methods, University of Milano-Bicocca Milan, Italy

Objective: to investigate both muscular manifestations and CK levels of a large cohort of patients with COVID-19 infection and to determine whether hyperckemia is associated with morbidity and mortality.

Methods: Data of 615 patients discharged from ASST Ovest Milanese (Milan, Lombardy, Italy) with final diagnosis of COVID-19 infection were retrospectively extracted from electronical medical records from 21 February to 1 May 2020. Patients were descriptively analyzed with respect to the following variables: sex, age, muscular manifestations (including myalgia/arthralgia and fatigue), respiratory involvement (SARS pneumonia or respiratory failure) and history of falls. Association between patients' characteristics and CK levels was investigated. In addition, the proportion of patients who died following access to the ER was calculated. Finally, the effect of CK levels and other patients' features on mortality was estimated using a logistic regression model.

Results: 176 (28.6\%) patients had raised serum CK levels. 88 (14.3\%) had muscular manifestations, of which $81(13.2 \%)$ complained fatigue and $17(2.8 \%)$ had myalgia and/or arthralgia. CK levels were significantly associated with respiratory involvement and fatal outcome. 
Conclusions: Our study provides preliminary evidence that CK levels are predictors of respiratory involvement and fatal outcome in patients with COVID-19 infection. For patients with muscle damage symptoms, screening for COVID-19 infection is recommended together with the dosage of CK level.

\section{Natural history course of DNM2-related congenital centronuclear} myopathy : a retrospective multicentre Italian Study

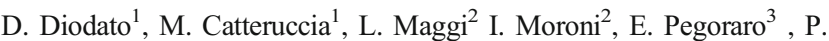
Riguzzi $^{3}$, L. Ruggiero ${ }^{4}$, M. Garibaldi ${ }^{5}$, A. Berardinelli ${ }^{6}$, M. Pane ${ }^{7}$, G. Atrea $^{8}$, F. Santorelli ${ }^{8}$, E. Bertini ${ }^{1}$, A. D'Amico ${ }^{1}$

${ }^{1}$ Ospedale Pediatrico Bambino Gesù, Roma, Italy

${ }^{2}$ Istituto Neurologico Carlo Besta, Milano, Italy

${ }^{3}$ Università di Padova, Italy

${ }^{4}$ Università Federico II, Napoli, Italy

${ }^{5}$ Ospedale Sant'Andrea, Roma, Italy

${ }^{6}$ IRCCS Fondazione Istituto Neurologico Nazionale C. Mondino, Pavia, Italy

${ }^{7}$ Policlinico Gemelli, Roma, Italy

${ }^{8}$ Fondazione Stella Maris, Pisa, Italy

Backgorund: Dynamin 2 (DNM2 gene) mutations are responsible of autosomal dominant form of centronuclear myopathy (CNM). DNM2-related CNMs can present with a wide severity spectrum and can range from neonatal onset forms to mild clinical picture in adults. CNMs due to DNM2 mutations are usually milder than the X-linked forms caused by mutations in myotubularin (MTM1) gene, and the autosomal recessive forms caused by mutations in the membrane-bending protein amphiphysin 2 (BIN1). Clinical picture of DNM2-related CNMs is mainly characterized by facial weakness, ptosis and ophthalmoparesis, distal muscle atrophy, joint contractures and pes cavus.

Cohort description: Here we present preliminary data of long-term follow-up of 18 patients with DNM2-CNMs. Age at diagnosis ranged from 5 to 48 years. The range of follow-up was from 2 to 30 years. The mean follow-up was of 10 years. We analysed a complete set of clinical data including respiratory function (FVC), motor function, scoliosis, nutritional status (dysphagia and/or the need of gastrostomy).

Results: We collected FVC follow-up data in 10/18 patients; in $8 / 10$ patients respiratory function remained stable in a mean period of 5 years. $5 / 18$ patients lost the ambulation at last visit of follow-up; $2 / 5$ patients who lost the ambulation presented with early-onset more severe form. Scoliosis data were available for $16 / 18$ patients; $3 / 16$ developed scoliosis in follow-up, 2/16 presented scoliosis at first evaluation. 5/18 patients presented with dysphagia at first evaluation, whereas $1 / 13$ developed dysphagia at follow-up. Further analysis of clinical data is needed to better delineate natural history of DNM2-CNMs patients.

Skin biopsy may help to distinguish Multiple System Atrophyparkinsonism type from Parkinson disease with orthostatic hypotension

V. Donadio ${ }^{1}$, A. Incensi ${ }^{1}$, G. Rizzo ${ }^{1,}{ }^{2}$, R. De Micco ${ }^{3}$, A. Tessitore ${ }^{3}$, G. Devigili $\mathrm{G}^{4}$, F. Del Sorbo ${ }^{4}$, S. Bonvegna ${ }^{4}$, M. Magnani $\mathrm{M}^{1}$, C. Zenesini ${ }^{1}$, L. Vignatelli ${ }^{1}$, R. Cilia ${ }^{4}$, R. Eleopra R ${ }^{4}$, G. Tedeschi ${ }^{3}$, R. Liguori ${ }^{1,2}$

${ }^{1}$ IRCCS Istituto delle Scienze Neurologiche di Bologna, UOC Clinica Neurologica, Italia

${ }^{2}$ Dipartimento di Scienze Biomediche e Neuromotorie, Università di Bologna, Italia

${ }^{3}$ Dipartimento di Scienze Mediche e Chirurgiche Avanzate, Università della Campania Luigi Vanvitelli, Napoli, Italia

${ }^{4}$ Fondazione IRCCS Istituto Neurologico Carlo Besta, Milano, Italia
Background: No established diagnostic tests are available to help the differential diagnosis between Multiple System Atrophy parkinsonism type (MSA-P) and Parkinson disease with orthostatic hypotension (PD+ $\mathrm{OH})$ showing a similar clinical picture but different prognosis. This study aimed at distinguishing MSA-P from $\mathrm{PD}+\mathrm{OH}$ by means of searching for phosphorylated $\alpha$-synuclein at serine 129 (p-syn) in skin nerves.

Methods: We recruited 50 patients with parkinsonism and chronic orthostatic hypotension: 25 patients fulfilling diagnostic criteria for MSA-P and 25 patients for $\mathrm{PD}+\mathrm{OH}$. Patients underwent skin biopsy from cervical, thigh and leg to analyse somatic and autonomic skin innervation and p-syn in skin nerves.

Results: The intraneural p-syn positivity was found in $72 \%$ of MSA-P patients, mainly in distal skin site. Importantly, p-syn deposits in MSA-P differ from $\mathrm{PD}+\mathrm{OH}$ since they were mainly found in somatic fibers of subepidermal plexuses, while scant autonomic fibers were involved in only 3 patients. On the other hand, all patients with $\mathrm{PD}+\mathrm{OH}$ displayed p-syn deposits in autonomic skin fibers widely distributed in proximal and distal skin sites, whereas scarce somatic fibers affected only 4 PD+ $\mathrm{OH}$ patients. Skin innervations mirrored p-syn deposits since epidermal somatic innervation was mainly affected in MSA-P while sympathetic denervation was found in $\mathrm{PD}+\mathrm{OH}$ but fairly preserved in MSA-P.

Conclusions: 1) p-syn in skin nerves allows differentiating MSA-P from $\mathrm{PD}+\mathrm{OH}$; 2) MSA-P shows a skin nerve involvement mainly affecting somatic fibers with relatively preserved autonomic innervation; 3 ) by contrast, $\mathrm{PD}+\mathrm{OH}$ display a prevalent autonomic postganglionic damage mainly affected by abnormal p-syn deposits.

Abnormal $\alpha$-synuclein deposits in skin nerves: inter and intralaboratory reproducibility

V. Donadio ${ }^{1}$, K. Doppler ${ }^{2}$, A. Incensi ${ }^{1}$, A. Kuzkina ${ }^{2}$, A. Janzen ${ }^{3}$, J. Volkmann $^{2}$, G. Rizzo ${ }^{1}$, E. Antelmi ${ }^{1}$, G. Plazzi ${ }^{1}$, C. Sommer ${ }^{2}$, WH Oertel $^{3}$, R. Liguori ${ }^{1}$

${ }^{1}$ IRCCS Institute of Neurological Sciences, Bologna, Italy

${ }^{2}$ Department of Neurology, University Hospital Würzburg, Würzburg, Germany.

${ }^{3}$ Philipps University, Marburg, Germany

Objective: phosphorylated $\alpha$-synuclein at serine 129 (p-syn) in skin nerves is a promising test for the in vivo diagnosis of synucleinopathies. Here we aimed to establish the intra and inter-laboratory reproducibility of intraneural p-syn positivity in two laboratories (Würzburg, Germany and Bologna, Italy) with a major expertise in this analysis.

Methods: we enrolled 42 patients (26 from Würzburg and 16 from Bologna) affected by Parkinson's disease (PD: 21 patients), REM sleep behavior disorder (RBD: 11), Multiple System Atrophy (MSA: 4) and small fiber neuropathy (SFN: 6). Skin biopsy was performed in C7 paravertebral spine region and distal leg. The analysis was standardized in both laboratories and made blinded on a single skin slide double stained with p-syn and PGP 9.5 (pan-neuronal marker). Fifty skin slides were analysed. Slides differently classified were re-evaluated to understand the reasons of the discrepancy.

Results: The intra-laboratory analysis showed an excellent reproducibility both in Würzburg (concordance of classification $100 \%$ of slides; $\mathrm{K}=1$; $\mathrm{p}<0.001)$ and Bologna (96\% of slides; $\mathrm{K}=0.92 ; \mathrm{p}<0.001)$. Interlaboratory analysis showed a reproducibility in 45 slides $(90 \% ; \mathrm{K}=0.8$; $\mathrm{p}<0.001$ ) and a different classification in 5 slides which was mainly due to fragmented skin samples or weak PGP 9.5 signal.

Conclusions: 1) p-syn analysis showed an excellent inter and intralaboratory reproducibility supporting the reliability of this technique as in vivo biomarker for synucleinopathies; 2) the few ascertained discordances were important to further improve the standardization of this technique. 
Sub cutaneous Immunoglobulin in two patients affected by Stiff person syndrome: a comparison study with intravenous Immunoglobulin

V. Donadio ${ }^{1}$, E. Fileccia ${ }^{1}$, Rinaldi R ${ }^{2}$, G.M. Minicuci ${ }^{3}$, R. D'Angelo ${ }^{3}$, L. Bartolomei ${ }^{4}$, Liguori $\mathrm{R}^{1,5}$.

${ }^{1}$ IRCCS Istituto delle Scienze Neurologiche di Bologna, UOC Clinica Neurologica, Ospedale Bellaria, Bologna, Italia

${ }^{2}$ IRCCS Istituto delle Scienze Neurologiche di Bologna, Clinica Neurologica Rete Metropolitana, Bologna, Bologna, Italy

${ }^{3}$ Unità Operativa Complessa di Neurologia, Ospedale 'San Bortolo', Vicenza, Italia

${ }^{4}$ Unità Operativa Complessa di Neurologia, Ospedale di Mirano, Mirano (Ve), Italia

${ }^{5}$ Dipartimento di Scienze Biomediche e Neuromotorie, Università di Bologna, Bologna, Italia

Introduction: Stiff person syndrome (SPS) is a rare condition characterised by either episodic spasms, prolonged stiffness or both. Immunoteraphy relies mainly on intravenous Immunoglobulin (IVIG); steroids, plasma exchange, azathioprine or rituximab are other possible options. We described two patients who showed a response to subcutaneous Immunoglobulin (SCIG) equivalent to intravenous admninistration.

Patient 1: a 46 year-old lady started to complain episodes of stiffness and spasms in her left lower limb which progressively affected the contralateral leg in a few months. At 49 year-old she received a diagnosis of Stiff Person syndromes (SPS) based on her clinical symptoms and serum and CSF dosage of Anti Glutamic acid decarboxylase (GAD) (CSF level 33 $\mathrm{UI} / \mathrm{ml}, \mathrm{nv}<1$, serum level $>2000 \mathrm{UI} / \mathrm{ml}, \mathrm{nv}<5)$. She started IVIG $(0,4$ $\mathrm{g} / \mathrm{kg} /$ day per 2 days every month) with clinical benefit. After 6 years a conversion to SCIG form was proposed. Her previous Intravenous dosage was converted to a monthly total of $60 \mathrm{~g}$ of SCIG. Before switching from intravenous to subcutaneous form, spasm frequency score (assessed by spasm frequency scale) was 2 and her modified Ashworth scale was 1 . She complained of low back and lower limbs pain (mean NRS 5/10 with sporadic peaks of 8/10) and her 10 meter time walk test was 6 seconds and muscle strengths assessed with MRC scale was normal. At 1 year follow-up from the switching her symptoms and scoring were stable.

Patient 2: a 46 year-old lady started to complain episodes of axial and lower limbs stiffness and spasms, which occurs more frequently at night. After three years of persistent symptoms and unrevealing examinations she underwent serum dosage of anti GAD antibodies and EMG testing which led to diagnosis of SPS. Treatment with IVIG was started $(0,4 \mathrm{~g} /$ day per 5 days, three cicle every two month) and her clinical symptoms substantially improved. At 51 year-old the patient was switched to SCIG at dosage of $100 \mathrm{~g} /$ month. Before switching her modified Ashworth scale was 1, her spasm frequency scale was between 1 and 2 and her timed 25 foot walk was 5,5 " on average. The patient did not complain pain, and her muscle strength assessed by MRC was normal. After one year of SCIG the patient's symptoms were stable, so were her scoring on scales and timed 25 foot walk. Conclusion:Subcutaneous formulation of Immunoglobulin could be as effective as Intravenous Immunoglobuline in maintenance treatment of SPS although studies involving a larger cohort of patients are needed in order to confirm our anecdotal experience with these two patients.

\section{REFERENCES}

1) José Fidel Baizabal-Carvallo- The neurological syndromes associated with glutamic acid decarboxylase antibodies. Journal of Autoimmunity 101 (2019) 35-47. doi.org/10.1016/j.jaut.2019.04.007)

2)Abbatemarco JR, Willis MA, Wilson RG, Nagel SJ, Machado AG, Bethoux FA. Case Series: Intrathecal Baclofen Therapy in Stiff-Person Syndrome. Neuromodulation. 2018 Oct;21(7):655-659. doi: 10.1111/ ner.12765. Epub 2018 Mar 13.)

3)M.C. Dalakas, M. Fujii, M. Li, B. Lutfi, J. Kyhos, B. McElroy, Highdose intravenous immune globulin for stiff-person syndrome, N. Engl. J. Med. 345 (2001)1870-1876.
Prevalence and relevance of diabetes mellitus in chronic inflammatory demyelinating polyneuropathy

P.E. Doneddu ${ }^{1}$, D. Cocito ${ }^{2}$, F. Manganelli ${ }^{3}$, R. Fazio ${ }^{4}$, C. Briani ${ }^{5}$, M. Filosto $^{6}$, L. Benedetti ${ }^{7}$, E. Bianchi ${ }^{8}$, S. Jann ${ }^{9}$, A. Mazzeo ${ }^{10}$, G. Antonini $^{11}$, G. Cosentino ${ }^{12}$, G.A. Marfia ${ }^{13}$, A. Cortese ${ }^{12}$, A.M. Clerici ${ }^{14}$ M. Carpo ${ }^{15}$, A. Schenone ${ }^{16}$, G. Siciliano ${ }^{17}$, M. Luigetti ${ }^{18,19}$, G. Lauria ${ }^{20,21}$, T. Rosso ${ }^{22}$, G. Cavaletti ${ }^{23}$, E. Beghi $^{8}$, G. Liberatore ${ }^{1}$, L. Santoro ${ }^{3}$, E. Spina $^{3}$, E. Peci ${ }^{2}$, S. Tronci ${ }^{4}$, M. Ruiz ${ }^{5}$, S. Cotti Piccinelli ${ }^{6}$, E.P. Verrengia $^{9}$, L. Gentile ${ }^{10}$, L. Leonardi ${ }^{11}$, G. Mataluni ${ }^{13}$, L. Piccolo ${ }^{12}$, E. Nobile-Orazio $^{1,24}$, on the behalf of the Italian CIDP Database Study Group.

${ }^{1}$ Neuromuscular and Neuroimmunology Service, Humanitas Clinical and Research Institute, Rozzano, Milan, Italy

2 Department of Neuroscience, University of Turin, Turin, Italy

3 Department of Neuroscience, Reproductive Sciences and Odontostomatology, University of Naples 'Federico II', Naples, Italy

4 Division of Neuroscience, Department of Neurology, Institute of Experimental Neurology (INSPE), San Raffaele Scientific Institute, Milan, Italy

${ }^{5}$ Neurology Unit, Department of Neuroscience, University of Padua, Padua, Italy

${ }^{6}$ Center for Neuromuscular Diseases and Neuropathies, Unit of Neurology, ASST 'Spedali Civili', University of Brescia, Brescia, Italy

${ }^{7}$ IRCCS Ospedale Policlinico San Martino Genova

${ }^{8}$ Laboratorio di Malattie Neurologiche, IRCCS-Istituto Mario Negri, Milan, Italy

${ }^{9}$ Department of Neuroscience, Niguarda Ca' Granda Hospital, Milan, Italy

10 Department of Clinical and Experimental Medicine, Unit of Neurology, University of Messina, Messina, Italy

${ }^{11}$ Unit of Neuromuscular Diseases, Department of Neurology Mental Health and Sensory Organs (NESMOS), Faculty of Medicine and Psychology, 'Sapienza' University of Rome, Sant'Andrea Hospital, Rome, Italy

${ }^{12}$ University of Pavia, Pavia, Italy. IRCCS Mondino Foundation, Pavia, Italy

${ }^{13}$ Dysimmune Neuropathies Unit, Department of Systems Medicine, Tor Vergata University of Rome, Rome, Italy

${ }^{14}$ Neurology Unit, Circolo \& Macchi Foundation Hospital, Insubria University, DBSV, Varese, Italy

${ }^{15}$ ASST Bergamo Ovest-Ospedale Treviglio, Treviglio, Italy

${ }^{16}$ Department of Neuroscience, Rehabilitation, Ophthalmology, Genetics, Maternal and Child Health, University of Genoa and IRCCS AOU San Martino-IST, Genoa, Italy

${ }^{17}$ Neurology Unit, Department of Clinical and Experimental Medicine, University of Pisa, Pisa, Italy

${ }^{18}$ Fondazione Policlinico Universitario Agostino Gemelli IRCCS. UOC Neurologia

${ }^{19}$ Università Cattolica del Sacro Cuore. Sede di Roma

${ }^{20}$ Unit of Neuroalgology, IRCCS Foundation 'Carlo Besta' Neurological Institute, Milan, Italy

${ }^{21}$ Department of Biomedical and Clinical Sciences 'Luigi Sacco', University of Milan, Milan, Italy

${ }^{22}$ ULSS2 Marca Trevigiana, UOC Neurologia-Castelfranco Veneto, Treviso, Italy

${ }^{23}$ School of Medicine and Surgery and Experimental Neurology Unit, University of Milano-Bicocca, Monza, Italy

${ }^{24}$ Department of Medical Biotechnology and Translational Medicine, Milan University, Milan, Italy

To determine the prevalence and relevance of diabetes mellitus (DM) in chronic inflammatory demyelinating polyradiculoneuropathy (CIDP). Using a structured questionnaire we collected information on DM from 393 CIDP patients fulfilling the EFNS/PNS criteria included in the Italian CIDP database. Frequency of DM in CIDP was compared with the 
estimated age- and gender-specific prevalence rates of DM in Italy. Fulfillment of the recently proposed diagnostic criteria of CIDP for the patients with DM was assessed. DM was significantly more frequent in CIDP patients than expected in the general Italian population (SPR 1.66; 95\% CI 1.31-2.07). Mean score of the diagnostic criteria for CIDP in DM among our patients with CIDP-DM was 12 (range 1-18; $\mathrm{SD} \pm 3.6$ ), with only one patient with a score below 2 points. Mean age of onset of DM was 51 years $(\mathrm{SD} \pm 13$ ), and $51 \%$ of the patients with $\mathrm{DM}$ were treated with oral antidiabetic therapy alone, $12.5 \%$ with insulin therapy alone, and $34 \%$ were not receiving therapy, suggesting a higher frequency of type 2 DM compared type 1 . Patients with DM had an older age at symptoms onset, more frequent signs of autonomic impairment, increased CSF proteins levels, higher disability by RODS and INCAT, worse QoL, and a less frequent response to treatment compared to patients without. DM is more frequent in CIDP than in the general population and type $2 \mathrm{DM}$ seems to be the most frequently associated type. DM seems to have an impact on disease severity and treatment response.

Home monitoring of different outcome measures during dose adjustment of intravenous immunoglobulin (IVIg) in patients with chronic inflammatory neuropathies

P. Doneddu ${ }^{1}$, D. Mandia ${ }^{1}$, F. Gentile ${ }^{1}$ F. Gallia ${ }^{1}$, G. Liberatore ${ }^{1}$, F. Terenghi $^{1}$, M. Ruiz, E. Nobile-Orazio ${ }^{1}$

${ }^{1}$ Humanitas Clinical and Research Institute, Milan, Italy

To determine the utility of home-based outcome measures (OM) during IVIg dosage adjustment in patients with chronic inflammatory neuropathies (CINs). Twenty patients (10 CIDP, $10 \mathrm{MMN}$ ) treated with regular IVIg were included. IVIg dose was adjusted to find the minimum effective dose. Patients were monitored with Martin-Vigorimeter (assessed daily), R-ODS (weekly) and SF-36 scale (daily) performed by the patients themselves and with the MRC sumscore on 26 muscles assessed at the hospital at the time of IVIg infusions. Thirty healthy controls were enrolled to measure any possible training effect of improvement with time and to analyze random fluctuation of grip-strength. Daily grip-strength deviated from baseline by $\geq 8 \mathrm{kPa}$ in $21(70 \%)$, by $\geq 10 \mathrm{kPa}$ in $17(57 \%)$, by $\geq 12 \mathrm{kPa}$ in $14(47 \%)$, and by $\geq 14 \mathrm{kPa}$ in 10 (33\%) controls, but deviated by $\geq 14 \mathrm{kPa}$ for more than three consecutive days only in 3 $(10 \%)$ controls. Controls grip-strength did not improve. Thirteen patients deteriorated after IVIg dose reduction, as confirmed by the MRC sumscore in 11 , by the R-ODS in 8 , and by grip-strength in 6 . No OM alone was sufficient to detect significant changes in all patients. In 5 patients IVIg therapy was suspended without clinical deterioration, as confirmed by all the OM apart RODS, which showed fluctuations in 2 patients. In 2 patients reporting end-of-dose effect, all OM objectively demonstrated improvement after IVIg dose increase. Home monitoring of different OM objectively assisted clinical decision on IVIg dose adjustments in patients with CINs.

\section{An Italian Database to assess the diagnosis, pathogenesis and effect of therapy in Multifocal Motor Neuropathy (MMN) and its variants: a prospective collaborative study}

P.E. Doneddu ${ }^{1}$, D. Cocito $^{2}$, A. Mazzeo ${ }^{3}$, L. Benedetti, ${ }^{4}$ M. Luigetti ${ }^{5}$, Fazio, ${ }^{6}$ C. Briani ${ }^{7}$, G. Siciliano, ${ }^{8}$ M.Filosto, ${ }^{9}$ G. Antonini, ${ }^{10}$ G. Cosentino $^{11}$, R. F. Manganelli, ${ }^{12}$ M. Inghilleri, ${ }^{13}$ M. Carpo, ${ }^{14}$ G.A. Marfia, ${ }^{15}$ G.M Minicuci, ${ }^{16}$ L. Gentile ${ }^{3}$, E. Peci ${ }^{2}$, A. Schenone, ${ }^{4}$ M. Sabatelli ${ }^{5}$, Tronci S, ${ }^{6}$ M. Campagnolo ${ }^{7}$, Schirinzi E, ${ }^{8}$ G. Liberatore ${ }^{1}$, E. Nobile-Orazio $^{1}$ for the MMN Study Group

${ }^{1}$ Neuromuscular and Neuroimmunology Service, IRCCS Humanitas Research Hospital, Milan University, Rozzano, Milan, Italy
${ }^{2}$ Presidio Sanitario Major, Istituti Clinici Scientifici Maugeri, Torino, Italy ${ }^{3}$ Department of Clinical and Experimental Medicine, Unit of Neurology, University of Messina, Messina, Italy

${ }^{4}$ Department of Neuroscience, Rehabilitation, Ophthalmology, Genetics, Maternal and Child

Health, University of Genoa, IRCCS San Martino, Genoa, and Neurology Unit Sant'Andrea Hospital, La Spezia Italy

${ }^{5}$ UOC Neurologia, Fondazione Policlinico Universitario A. Gemelli IRCCS, Università Cattolica del Sacro Cuore. Rome, Italy

${ }^{6}$ Division of Neuroscience, Department of Neurology, Institute of Experimental Neurology (INSPE), San Raffaele Scientific Institute, Milan, Italy

${ }^{7}$ Neurology Unit, Department of Neuroscience, University of Padua, Padua, Italy

${ }^{8}$ Neurology Unit, Department of Clinical and Experimental Medicine, University of Pisa, Pisa, Italy

${ }^{9}$ Center for Neuromuscular Diseases and Neuropathies, Unit of Neurology, ASST 'Spedali Civili', University of Brescia, Brescia, Italy

${ }^{10}$ Department of Neurology Mental Health and Sensory Organs (NESMOS), 'Sapienza' University of Rome, Sant'Andrea Hospital, Rome, Italy

${ }^{11}$ Neurology, IRCCS Fondazione Mondino, Pavia University, Pavia, Italy

12 Department of Neuroscience, Reproductive Sciences and Odontostomatology, University of Naples 'Federico II', Naples, Italy

${ }^{13}$ Neurodegenerative Disorders Unit, Department of Human Neuroscience, 'Sapienza' University of Policlinico Universitario Umberto I, Rome

${ }^{14}$ Neurology Unit, ASST Bergamo Ovest-Ospedale Treviglio, Treviglio, Italy

${ }^{15}$ Dysimmune Neuropathies Unit, Department of Systems Medicine, Tor Vergata University of Rome, Rome, Italy

${ }^{16}$ Neurological Institute, 'San Bortolo' Hospital, Vicenza

MMN is a purely motor neuropathy with asymmetric limb weakness and conduction blocks (CB) on motor nerves. Several data point to a pathogenetic role of the immune system in MMN including the frequent occurrence of anti-GM1 IgM antibodies and response to immunoglobulin (IVIg). The cause of MMN remains unclear. CB is the main diagnostic feature of MMN even if there are patients with clinically typical MMN responding to IVIg without detectable CB. This led to some confusion on the distinction between MMN and other lower motor neuron syndromes often leading to an inappropriate use of IVIg. The aim of this project is to analyze and prospectively follow patients with a clinical diagnosis of MMN to define the diagnostic criteria of this neuropathy including the role of nerve conduction studies and laboratory tests, and to analyze the presence of possible antecedent events and associated diseases. We included so far 80 patients (56 men, 24 women) with a mean age at disease onset of 41.4 years. An initial selective involvement of upper limbs was reported by $69 \%$ of the patients while $13 \%$ had a concomitant lower limb impairment and $18 \%$ of lower limbs only. At the time of inclusion, patients had a mean disease duration of 11.9 years and ONLS score of 3.3, with a persistent selective upper limb involvement in $44 \%$ and of lower limb in $2 \%$. $86 \%$ of treated patients improved after IVIg. All the data available at the time of the congress will be presented. Supported by Baxalta/Shire.

Alpha-sarcoglycanopathy presenting as myalgia and hyperckemia in two adults with a long-term follow-up

C. Dosi ${ }^{1}$, A. Rubegni ${ }^{1}$, D. Cassandrini ${ }^{1}$, A. Malandrini ${ }^{2}$, L. Maggi ${ }^{3}$, FM. Santorelli ${ }^{1}$

${ }^{1}$ IRCCS Fondazione Stella Maris, Pisa, Italy

${ }^{2}$ Department of Medicine, Surgery and Neurosciences, University of Siena, Siena, Italy 
${ }^{3}$ Fondazione IRCCS Istituto Neurologico Carlo Besta, Milano, Italy

The advancing information in the field of muscle disorders are leading to the identification of previously unrecognized causes of hyperCKemia associated with mild neurological signs. Few studies have reported hyperCKemia as the presenting feature of a sarcoglycanopathy in association with myoglobinuria, exercise induced myalgias and/or rhabdomyolysis.

In a recent cohort study of undiagnosed patients with hyperCKemia using multiple gene panel with methodologies of next-generation sequencing (NGS), we detected a subset of paucisymptomatic individuals harboring biallelic variants in known muscular dystrophy genes. We herein discuss two patients (a 31-year-old woman and a 36-year-old man) who showed occasional exercise intolerance, myalgia and high serum CK levels (meeting the EFNS criteria for hyperCKemia), in the absence of other neurological signs and harbored biallelic variants of predicted pathogenic significance in $S G C A$. Interestingly, over their 25 -year follow-up they have remained oligosymptomatic presenting with minimal muscle weakness. In both cases, muscle biopsy showed minimal changes and $\alpha$-sarcoglycan labeling showed normal immunofluorescence in the man and a slight reduction in the woman. Although reports of mild presentations of sarcoglycanopathies have already been discussed before, our cases add to the diagnostic dilemma faced by a clinician dealing with a patient presenting with recurrent myalgias or a mildly elevated CK. NGS methodologies combined with muscle biopsy examination remain a cost effective diagnostic tool in patients featuring non-specific or uncertain skeletal myopathy.

\section{Identification and characterization of urine-derived stem cells} (USCs) by the novel technology $\operatorname{Celector}{ }^{\circledR}$

M. S. Falzarano ${ }^{1}$, N. Spedicato ${ }^{1}$, A. Margutti ${ }^{1}$, R. El Dani ${ }^{1}$, R. Rossi ${ }^{1}$, S. $\mathrm{Zia}^{2}$, P. Reschiglian ${ }^{2,3}$ B. Roda ${ }^{2,3}$, A. Grilli ${ }^{4}$, S. Bicciato ${ }^{4}$, A. Ferlini ${ }^{1}$

${ }^{1}$ University of Ferrara, Ferrara, Italy;

${ }^{2}$ Stem Sel s.r.l., Bologna, Italy;

${ }^{3}$ University of Bologna, Bologna, Italy;

${ }^{4}$ University of Modena and Reggio, Modena, Italy.

Urine-derived stem cells (USCs) are known to be a very promising source of stem cells, in addition their isolation is non-invasive, relatively quick and simple, affordable, ubiquitous, and readily accepted by patients.

The USCs transcriptional profiling showed a great variability across individuals probably due to the co-presence of distinct cell types. The lack of specific and unique cell surface markers for the identification of each cell types makes the separation difficult.

In order to separate the USCs sub-populations, we used the new technology Celector ${ }^{\circledR}$ that sorts the cells based only on their physical characteristics.

Urine samples were collected from 3 controls and two Duchenne muscular Dystrophy (DMD) patients carrying the deletion of exon 50-52 and exon 46-47, respectively.

Celector separation profiled USCs as heterogeneous population composed by at least 3 different cell fractions. Control USCs showed a reproducible elution profile and similar expression of CD70, CD90, and CD105 mesenchymal markers. Conversely, DMD-USCs exhibited two distinct profiles compared to controls. No morphological differences were observed among fractions in all samples.

The DMD isoforms were differentially detected among each DMD fraction and between the two DMD patients.

Our data provide an important and precious foundation for further studies to define the different properties of each fraction, especially in view of their use in direct differentiation and induced pluripotency reprogramming methods, and to create a large collection of immortalized USCs with prolonged proliferative capacity, useful for pathophysiological studies and novel therapeutic strategies.
An overlapping case of Myasthenia Gravis, Guillain-Barré Syndrome and Autoimmune Polyglandular Syndrome type III

L. Ferri ${ }^{1}$, MG Rispoli ${ }^{1}$, V. Falzano ${ }^{2}$, P. Ajdinaj $^{1}$, V. Di Stefano ${ }^{3}$, A. Di Muzio ${ }^{4}$

${ }^{1}$ Department of Neuroscience Imaging and Clinical Sciences, Gabriele d'Annunzio University of Chieti and Pescara, Chieti, Abruzzo, Italy

${ }^{2}$ Department of Endocrinology, SS Annunziata Hospital, Chieti, Abruzzo, Italy

${ }^{3}$ Department of Biomedicine, Neuroscience and advanced Diagnostic, University of Palermo, Palermo, Sicily, Italy

${ }^{4}$ Department of Neurology, SS Annunziata Hospital, Chieti, Abruzzo, Italy

The occurrence of Myasthenia Gravis (MG) and Guillain-Barré Syndrome (GBS) in the same patient is rare ${ }^{1}$. Frequency of autoimmune comorbidities is about $25-78 \%$ in MG patients ${ }^{2}$.

In January 2015, a 24-year-old female was admitted to our hospital because of acute appearance of lower limbs weakness, dysesthesias, areflexia and difficulties in walking occurred two months before. EMG disclosed increased motor latency, decreased conduction velocity and absence of $\mathrm{F}$ waves in the lower limbs and GBS was diagnosed.

In April 2015 she had the onset of diplopia and ptosis and started pyridostigmine with beneficial effects. A $3 \mathrm{~Hz}$ repetitive nerve stimulation in trapezius showed a $10 \%$ decrement and a diagnosis of MG was established. No thymic abnormalities were detected on the chest MRI.

Because she also presented chronic lymphocytic-thyroiditis, with positivity of thyroid peroxidase and anti-thyroglobulin antibodies, systemic sclerosis, secondary amenorrhea and positivity of anti-parietal-cell antibodies, a type III autoimmune polyendocrine syndrome (APSIII) was then diagnosed ${ }^{3}$.

In April 2016 she had worsening of myasthenic symptoms, with diplopia, ptosis and fluctuating limb weakness and intravenous immunoglobulin infusion was performed, with global improvement but easy relapsing of symptoms. So she started Azathioprine with beneficial effects on all diseases with progressive normalization of thyroid function.

To our knowledge, coexistence of GBS, MG and APS III has never been reported. In this context, immunotherapy can be beneficial also on endocrine diseases.

1. Misra UK et al. Acta Neurologica Belgica. 2020;120(1):59-64.

2. Cao Y et al. Medicine. 2019;98(47):e18104.

3. Kahaly G. and Frommer L. Journal of endocrinological investigation. 2018:41(1):91-98.

Muscle MRI findings and correlation with clinical and immunological parameters in a cohort of IMNM patients

L.Fionda ${ }^{1}$, F.Vanoli ${ }^{2}$, L. Leonardi ${ }^{1}$, J.Alonso Perez ${ }^{2}$, J. Diaz Manera ${ }^{2}$, G. Merlonghi $^{1}$, S. Morino ${ }^{1}$, E. Bucci ${ }^{1}$, G. Alfieri ${ }^{1}$, A. Lauletta ${ }^{1}$, G. Antonini ${ }^{1}$, M. Garibaldi ${ }^{1}$

${ }^{1}$ Neuromuscular and Rare Disease Center, Department of Neuroscience, Mental Health and Sensory Organs (NESMOS), SAPIENZA University, Sant'Andrea Hospital, Rome, Italy.

${ }^{2}$ Neuromuscular Diseases Unit, Neurology Department, Hospital de la Santa Creu I Sant Pau and Biomedical Research Institute Sant Pau (IIB Sant Pau), Barcelona, Spain.

Introduction: Immune-mediated necrotising myopathies (IMNM) are characterized by progressive and severe muscle impairment, frequently associated to fatty replacement of muscular tissue that leads to a permanent deficit.

The aim of this study is to detect, through a whole body muscle MRI, the presence and the evolution of inflammation and muscular damage, and if 
there is a correlation with clinical and immunological aspects, and comparing response to different therapeutic strategies.

Materials and methods: Twenty-two patients were included. Age, disease duration, timing and type of therapy performed with respect to the moment of MRI acquisitions, were recorded for each patient.

Muscle imaging was examined using T1-TSE and STIR weighted sequences. A total of 39 muscles of lower limbs and 20 of scapular girdle and arm were analyzed. The presence of fatty infiltration was evaluated according to Fischer's semi-quantitative scale. The presence of edema/ inflammation of muscles was established on STIR sequences.

Results: At the moment of MRI acquisition, 8 anti-HMGCR, 7 anti-SRP and 7 seronegative IMNM were aged 60,1 17,3. Eight patients were without treatment, and disease duration was 5,8 87,8 years. Eleven patients underwent a second muscle MRI, after a median time of 15 months. Eleven patients needed an association therapy with immunosuppressors, while twelve underwent IVIG. Fourteen patients did not recover completely muscle strength. Age at onset, disease duration and anti-SRP positivity were associated with a more severe muscle involvement. Most affected muscles, according to T1-wheigted images, were lumbar paravertebral, gluteal, adductors and hamstring groups. Muscle edema /inflammation distribution seems to be a predictor of fatty replacement overtime.

UNC-45B mutations in congenital axial myopathy with cores and multiple internalized nuclei

C.Fiorillo ${ }^{1,2}$, C.Panicucci ${ }^{1}$, S. Donkervoort ${ }^{3}$, M.Traverso ${ }^{1}$, P.Broda ${ }^{1}$, R.Falsaperla $^{4}$, V.Salpietro ${ }^{1,2}$, C.Minetti ${ }^{1,2}$

${ }^{1}$ Pediatric Neurology and Muscular Diseases Unit, G. Gaslini Institute, 16147, Genoa, Italy;

${ }^{2}$ Department of Neurosciences, Rehabilitation, Ophthalmology, Genetics, Maternal and Child Health (DINOGMI), University of Genoa, 16132 Genoa, Italy;

${ }^{3}$ Neuromuscular and Neurogenetic Disorders of Childhood, National Institutes of Health, 20892, Bethesda, MD, USA,

${ }^{4}$ Department of Paediatrics, Policlinico-Vittorio Emanuele University Hospital, Catania, Italy

UNC-45B (unc45 myosin chaperone B) encodes a myosin-specific co-chaperone essential for the folding and maintenance of sarcomeric myosins, facilitating their proper assembly and function. In particular, UNC45B aggregates to a chain which serves as an assembly line for beta ( $\beta$ )-myosin heavy chain, encoded by MYH7.

Recently, biallelic mutations in UNC-45B have been described in 11-year old male with congenital myopathy and core-like lesions at muscle biopsy.

Here, we report a further case of Italian origin, in whom we identified by whole exome sequencing the recurrent $2261 \mathrm{G}>\mathrm{A}$, p.Arg754Gln inherited from the father and a novel c.1207T >C, p.Ser403Pro inherited from the mother. p.Arg754Gln is localised in the USC region of the gene which is critical for myosin binding, whereas the Ser403Pro impact the neck domain. Of interest, among other NGS identified variants, are 2 missense compound heterozygous in $M Y H 7$ gene and we question a putative phenotype modifier effect.

From a clinical point of view, the 5 years old female manifested motor delay and axial hypotonia in the first months of life. Ambulation was possible at 3 years with waddling gait and increased lumbar curve. Later she developed overt Gower's sign and limb girdle muscles wasting. Prominence of calcaneus and mild facial weakness were also present. Skeletal muscle biopsy revealed myopathic changes with central and eccentric cores, predominance of type I fibre and multiple internalised nuclei in nearly $50 \%$ of fibres.

We performed Western blot and Immunofluorescence analysis which revealed a severe reduction of UNC-45B protein in patient muscle compared to control and abnormal relocalization of the UNC-45B protein

Our case reinforce the role of recessive mutations in $U N C-45 B$ as a cause of a progressive myopathy and expand the clinical and histopathological phenotype.
Fatigue and sleepiness in myotonic dystrophy type 1: motor, neuropsychological and sleep correlates

E. Frezza ${ }^{1}$, C. Galluzzi ${ }^{1}$, G. Greco ${ }^{1}$, P. Proserpio ${ }^{2}$, L. Mauro ${ }^{3}$, G. Sannicolò $^{3}$, A. Pirola ${ }^{3}$, E. Falcier ${ }^{3}$, E. Roma ${ }^{3}$, A. Zanolini ${ }^{3}$, A. Lizio ${ }^{3}$, C. Liguori ${ }^{4}$, F. Placidi ${ }^{4}$, V.A. Sansone ${ }^{5,3}$, R. Massa ${ }^{1}$

${ }^{1}$ Neuromuscular Diseases Unit, Tor Vergata University of Rome

'Department of Neuroscience, Niguarda Hospital, Milan

The NEMO Clinical Center, Milan

Sleep Disturbances Unit, Tor Vergata University of Rome

Neurorehabilitation Unit, University of Milan

Introduction: Fatigue and Excessive Daytime Sleepiness (EDS) are commonly reported by Myotonic Dystrophy type 1 (DM1) patients. Although these terms are often used indistinctly by patients, they should be differentiated since they represent distinct symptoms. Therefore, we assessed the prevalence of fatigue and EDS, and evaluated their relationships with motor, neuropsychological and sleep characteristics in adult DM1.

Patients and methods: A cohort of $61 \mathrm{DM} 1$ patients of either sex underwent 6MWT, Timed up and go, 9 hole-peg test, MRC, neuropsychological evaluation (Stroop test, trail making test, Wisconsin card sorting test), and questionnaires: Epworth sleepiness scale (ESS), Fatigue Severity Scale (FSS), Hamilton depression rating scale, profile of mood states, SF-36, INQoL. Sleep characteristics were studied objectively by polysomnography, multiple sleep latency test and maintenance of wakefulness test.

Results: Patients reported: 1) neither EDS nor fatigue (29\%); 2) EDSonly $(7 \%)$; 3) fatigue-only $(37 \%)$; 4 ) both fatigue and EDS $(27 \%)$. No significant differences between groups were found for age, sex, BMI and mean CTGn. The fatigue-only group showed the worst performance at 6MWT $(p<0.05)$, whereas patients with both fatigue and EDS performed better. No differences were found concerning sleep alteration and neuropsychological tests. Depression and poor QoL were more pronounced in patients reporting fatigue, either isolated or with $\operatorname{EDS}(p<0.01)$.

Conclusion: In DM1, reported fatigue seems to have a muscular origin when isolated and a central origin when combined with EDS. Higher scores on the FSS are useful in identifying patients with higher burden on mood and greater impact on QoL.

Neurofilament light chain: a possible serum biomarker for axonal damage in chemotherapy-induced peripheral neurotoxicity rat models

G. Fumagalli ${ }^{1}$, A. Chiorazzi ${ }^{1}$, V. Rodriguez-Menendez ${ }^{1}$, K. Blennow ${ }^{2}$, ${ }^{3}$, H. Zetterberg ${ }^{2,3,4,5}$, G. Cavaletti ${ }^{1}$, P. Marmiroli ${ }^{1}$

${ }^{1}$ Experimental Neurology Unit and NeuroMI (Milan Center for Neuroscience), School of Medicine and Surgery, University of MilanoBicocca, Monza, Italy;

${ }^{2}$ Department of Psychiatry and Neurochemistry, the Sahlgrenska Academy at the University of Gothenburg, Mölndal, Sweden;

${ }^{3}$ Clinical Neurochemistry Laboratory, Sahlgrenska University Hospital, Mölndal, Sweden;

${ }^{4}$ Department of Neurodegenerative Disease, UCL Institute of Neurology, Queen Square, London, United Kingdom;

${ }^{5}$ UK Dementia Research Institute at UCL, London, United Kingdom

Nowadays it is difficult to monitor the onset and progression of chemotherapy-induced peripheral neurotoxicity (CIPN) in treated patients. The use of a serum biomarker is the easier and most reliable way for an early detection in order to avoid a permanent axonal damage. Neurofilaments are axonal prominent components and they are released into the blood after axonal damage. We performed serum neurofilament light (NfL) chain dosage as a possible biomarker in CIPN models. 
Wistar rats were divided in control (CTRL, untreated), cisplatin- (CDDP, $2 \mathrm{mg} / \mathrm{kg}$ i.p. 2 qwx $4 \mathrm{ws}$ ) and paclitaxel- (PTX, $10 \mathrm{mg} / \mathrm{kg}$ i.v. q7dx4 ws) treated groups. The axonal damage, evaluated by neurophysiological and morphological investigations, was correlated with serum NfL concentration measured using a Single molecule array (Simoa).

A significant reduction in sensory action potential amplitude and an important axonal loss were detected in caudal nerves of PTX-treated rats from the second week of treatment using neurophysiological and morphological examinations, respectively. A mild axonopathy was detected in CDDP-treated animals only at the end of the treatment.

Interestingly, serum NfL concentration correlated with the severity of the axonal damage: in PTX-treated animals the levels were high from early time point, whereas in CDDP-treated rats NfL concentration steadily increased, but it was lower than in PTX-treated rats.

In conclusion, $\mathrm{NfL}$ concentration correlated with the progression of the axonal damage and it was higher in PTX-compared to CDDP-treated group according to the more severe axonal damage, indicating the possible use of NfL as a serum biomarker for CIPN grading and severity.

\section{Congenital Myasthenic Syndromes: a large Italian cohort of patients}

A. Gallone ${ }^{1}$, R. Brugnoni ${ }^{1}$, A. Ardissone ${ }^{1}$, E. Terlizzi $^{2}$, R. Masson ${ }^{1}$, G. Ricci $^{3}$, F. Magri ${ }^{4}$, F. Guidolin ${ }^{5}$, M.L. Valentino ${ }^{6}$, D. Frattini ${ }^{7}$, C. Bonanno $^{8}$, M. Catteruccia ${ }^{9}$, A. Malandrini ${ }^{10}$, G. Primiano ${ }^{11}$, C. Antozzi $^{1}$, P. Confalonieri ${ }^{1}$, G. Tasca ${ }^{11}$, M. Monforte ${ }^{11}$, E. Ricci ${ }^{11}$, G. Astrea $^{12}$, C. Ticci $^{12}$, M. Garibaldi ${ }^{13}$, D. Orsucci ${ }^{14}$, F. Ricci ${ }^{15}$, M.T. Ferrò $^{16}$, V.A. Donadio ${ }^{6}$, A. Gentili ${ }^{17}$, E. Bertini ${ }^{9}$, G. Siciliano ${ }^{3}$, D. Piga $^{4}$, G.P. Comi ${ }^{4}$, R. Liguori ${ }^{6}$, I. Moroni ${ }^{1}$, F.M. Santorelli ${ }^{12}$, S. Servidei $^{11}$, A. D'Amico 9 , G. Antonini ${ }^{13}$, A. Evoli ${ }^{11}$, D. Sternberg ${ }^{18}$, D. Beeson $^{19}$, A. Engel ${ }^{20}$, H. Lochmüller ${ }^{21,22}$, R. Mantegazza ${ }^{1}$, P. Bernasconi $^{1}$, C. Rodolico ${ }^{8} *$ L. Maggi ${ }^{1} *$.

${ }^{1}$ Fondazione IRCCS Istituto Neurologico Carlo Besta, Milano, Italy

${ }^{2}$ Ospedale Guglielmo da Saliceto, Piacenza, Italy

${ }^{3}$ Università di Pisa, Pisa, Italy

${ }^{4}$ Fondazione IRCCS Ca' Granda Ospedale Maggiore Policlinico, Università di Milano, Milano, Italy

${ }^{5}$ IRCCS Burlo Garofolo, Trieste, Italy

${ }^{6}$ IRCCS Istituto delle Scienze Neurologiche di Bologna, Bologna, Italy

${ }^{7}$ AO - IRCCS - Arcispedale S. Maria Nuova, Reggio Emilia, Italy

${ }^{8}$ Università di Messina, Messina, Italy

${ }^{9}$ Ospedale Pediatrico Bambin Gesù IRCCS, Roma, Italy

${ }^{10}$ Università di Siena, Siena, Italy

${ }^{11}$ Fondazione Policlinico Universitario A. Gemelli IRCCS, Roma, Italy

${ }^{12}$ IRCCS Fondazione Stella Maris, Pisa, Italy

${ }^{13}$ Università La Sapienza, Ospedale Sant'Andrea, Roma, Italy

${ }^{14}$ Ospedale San Luca, Lucca, Italy

${ }^{15}$ Città della Salute e della Scienza di Torino, Torino, Italy

${ }^{16}$ Ospedale di Crema, Crema, Italy

${ }^{17}$ Università di Bologna, Bologna, Italy

${ }^{18}$ Groupe Hospitalier Pitié-Salpêtrière, Paris, France

${ }^{19}$ University of Oxford, Oxford, UK

${ }^{20}$ Mayo Clinic, Rochester, MN, USA

${ }^{21}$ University of Freiburg, Freiburg, Germany

${ }^{22}$ University of Ottawa, Ottawa, Canada

\section{*Both authors contributed equally to the work}

Background: Congenital myasthenic syndromes (CMS) are rare and heterogeneous diseases due to mutations in proteins involved in the structure or function of the neuromuscular junction. However, CMS natural history has not been fully elucidated and most of the studies in CMS include small cohort of patients. We aimed here to describe clinical, histological and molecular data in a large cohort of genetically defined CMS Italian patients.
Methods: We included patients with a molecular genetic diagnosis of CMS, followed up in 17 Italian Neuromuscular Centres.

Results: One hundred nine patients were found to carry pathogenic mutations: 44 (40.4\%) in CHRNE, $12(11 \%)$ in COLQ, 10 (9.2\%) in DOK7, 10 (9.2\%) in CHRNA1, 8 (7.3\%) in RAPSN, $6(5.5 \%)$ in GFPT1, $5(4.6 \%)$ in CHRND, $4(3.7 \%)$ in CHAT, $3(2.7 \%)$ in CHRNB1, $2(1.8 \%)$ in DAPTG1, $2(1.8 \%)$ in SCL5A7, $2(1.8 \%)$ in MUSK and $1(0.9 \%)$ in SYT2. Mean age at onset was 5.3 years (range $0-60)$. Twelve $(11 \%)$ patients presented after the age of 18 years: 4 mutated in CHRNA1, 4 in DOK7, 1 in CHRNE, 1 in SYT2, 1 in CHRNB1 and 1 in COLQ. Notably, all the patients mutated in CHRNA1 and the one in CHRNE presenting in adult age had a slow channel syndrome; further 9 patients displayed a slow channel syndrome in the early onset group.

Conclusions: Our data provide new insights in the field of CMS and further support the need of prospective studies to better clarify CMS natural history.

\section{Gender effect on cardiac involvement in Myotonic Dystrophy type 1 (DM1)}

M. Garibaldi ${ }^{1}$, L. Fionda ${ }^{1}$, E. Bucci ${ }^{1}$, F. Vanoli $^{1}$, Luca Leonardi ${ }^{1}$, Antonio Lauletta ${ }^{1}$, Girolamo Alfieri ${ }^{1}$, Gioia Merlonghi ${ }^{1}$, Stefania Morino ${ }^{1}$, Marco Testa ${ }^{2}$, G. Antonini ${ }^{1}$

${ }^{1}$ Neuromuscular and Rare Disease Center, Department of Neuroscience, Mental Health and Sensory Organs (NESMOS), SAPIENZA University, Sant'Andrea Hospital

${ }^{2}$ Department of Cardiology, Sapienza University of Rome, Sant'Andrea Hospital, Rome, Italy

Cardiac involvement is observed in about $80 \%$ of subjects with Myotonic Dystrophy type1 (DM1) and is mainly characterized by cardiac conduction (CCA) and/or rhythm (CRA) abnormalities (CCRA), possibly leading to sudden cardiac death (SDC). The aim of this study is to investigate whether the gender difference may influence the cardiac involvement and sudden cardiac death in DM1. We analyzed prevalence and incidence of cardiologic abnormalities in males versus females in 151 consecutive DM1 patients over 35 years of follow-up period. Fifty-five patients, 35 males $(62.5 \%)$ and 20 females $(42.5 \%)$ developed some type of CCRA during the follow-up period (mean $7.82 \pm 6.21$ years). CCRA overall, and specifically CCA, were significantly more frequent in males than in females $(p=0.043$ and $p=0.031$ respectively). CCRA progressed in 16 males $(45.7 \%)$ and 6 females $(30 \%)$. Twenty-four patients, 14 males $(25.0 \%)$ and 10 females $(21.3 \%)$ deceased during the follow-up. Nine of them, 6 males $(10.7 \%)$ and 3 females $(6.4 \%)$ had a SCD. After correction for MIRS progression, CTG expansion and follow-up duration, a higher prevalence of CCA was independently associated with male gender $(\mathrm{p}=0.039)$, but not independent association with gender was detected for CCRA overall, CRA and SCD prevalence, even if prevalence was higher in males than females. The overall risk of occurrence of CCA in DM1 is significantly higher in males than females regardless of genetical background and disease severity and progression. As well, the data suggest similar impact of male gender also for CCRA overall, CCA and $\mathrm{SCD}$ even if not statistically significant.

\section{Muscle MRI in Myotonic Dystrophy type1 (DM1)}

M. Garibaldi ${ }^{1}$, T. Nicoletti ${ }^{2}$, L. Fionda ${ }^{1}$, E. Bucci ${ }^{1}$, T. Tartaglione ${ }^{3}$, G. Tasca $^{2}$, A. Perna ${ }^{2}$, A. Petrucci ${ }^{4}$, G. Silvestri ${ }^{2}$, G. Antonini ${ }^{1}$

${ }^{1}$ Neuromuscular and Rare Disease Center, Department of Neuroscience, Mental Health and Sensory Organs (NESMOS), SAPIENZA University, Sant'Andrea Hospital 
${ }^{2}$ Fondazione Policlinico Universitario "A. Gemelli" IRCCS, Università Cattolica del Sacro Cuore, Roma

${ }^{3}$ Department of Radiology, Istituto Dermopatico dell'Immacolata, IRCCS, Rome

${ }^{4}$ Neurology Unit, San Camillo-Forlanini Hospital, Rome, Italy.

Disease-specific patterns of muscle involvement have been detected in several myopathies. Only few studies reported muscle imaging data on dozens of DM1 patients. The aim of this study is to redefine the muscle involvement in a large cohort of DM1 patients and to search a correlation with clinical and genetic data. Whole body muscle MRI was obtained by about 150 genetically confirmed DM1 patients. T1 and STIR sequences were considered in thirtytwo groups of muscles of lower limbs and 20 of neck-scapular girdle. The most frequent pattern of muscle involvement showed that multifidus, gluteus minimus, distal quadriceps and medial gastrocnemius were the most affected muscles. A subset of patients $(\sim 10 \%)$ did not show the typical pattern. Most of patients showed muscle atrophy in cervical muscles regardless of fibro-fatty replacement. More than $80 \%$ of patients had STIR positive muscles. The overall MRI severity correlated with clinical severity, but not with genetic background. A variable degree of muscle involvement was also detected in milder- asymptomatic patients (MIRS 1-2) suggesting the high sensitivity of MRI as a biomarker also for the milder spectrum of disease.

RNAi therapeutic Patisiran in hATTR amyloidosis: tolerability and management from two centre experience

L. Gentile $\mathrm{MD}^{1}$, M. Russo $\mathrm{MD} \mathrm{PhD}^{1}$, M. Luigetti $\mathrm{MD} \mathrm{PhD}^{3,4}$, G. Bisogni $\mathrm{MD}^{2}$, A. Di Paolantonio $\mathrm{MD}^{3,4}$, A. Romano $\mathrm{MD}^{2,3}, \mathrm{~V}$. Guglielmino $\mathrm{MD}^{3,4}$, M. Sabatelli $\mathrm{MD}^{2,3}$, A. Toscano $\mathrm{MD} \mathrm{PhD}^{1}, \mathrm{G}$. Vita $\mathrm{MD} \mathrm{PhD}^{1}$, A. Mazzeo MD $\mathrm{PhD}^{1}$.

${ }^{1}$ Unit of Neurology and Neuromuscular Diseases, Department of Clinical and Experimental Medicine, University of Messina, via Consolare Valeria 1, 98125, Messina, ME, Italy.

${ }^{2}$ Centro Clinico NEMO Adulti Roma, Italy

${ }^{3}$ Università Cattolica del Sacro Cuore. Sede di Roma, Italy

${ }^{4}$ Fondazione Policlinico A. Gemelli IRCCS. UOC Neurologia, Italy

Introduction: hATTR amyloidosis is a severe disease characterized by progressive axonal sensory-motor polyneuropathy with autonomic dysfunction and multiorgan involvement (heart, kidneys, eyes). Patisiran is a si-RNA able to degrade the m-RNA strand from TTR gene, reducing the synthesis of the mutated protein. A phase 3 trial demonstrated the improvement of symptomatology, measured by the modified Neuropathy Impairment Score+7 (NIS+ 7) and Norfolk Quality of Life Diabetic Neuropathy score (QOLDN), without significant adverse events except for mild infusion site reactions.

Materials and methods: Since 2014, we have treated with patisiran 23 patients, with the following TTR mutations: Phe64Leu (10), Glu89Gln (5), Val30Met (4), Thr49Ala (2), Val122Ile (1) and Ala109Ser (1). 4/23 patients were also in treatment with tafamidis. 19/23 were male. Age at first infusion: $48-78$ years. Seven patients were at FAP stage II and sixteen in FAP stage I. All patients were evaluated by NIS scale, QOL-DN score and Sudoscan at baseline and every 6 months.

Results: We observed a stabilization of clinical parameters during the therapy with mild improvements in some cases. We reported two side effects secondary to the use of premedication drugs (hyperglycemia and hypertension). Three patients temporarily discontinued the therapy because of hospitalizations respectively for severe anaemia, fever, and diarrhoea. We also reported three death: two patients presented a sudden death, probably of cardiac origin; one patient died as complication of severe dehydration. It is highly unlikely that these episodes are related to patisiran infusion.

Conclusions: Clinical stabilization was observed during patisiran therapy. Adverse events were correlated mainly to the use of premedical drugs.

Perivascular M1 macrophages expressing VEGF and SDF1 promote angiogenesis in anti-HMGCR immune mediate necrotizing myopathy

F. Girolamo ${ }^{1}$, M. Fornaro ${ }^{2}$, M. Giannini ${ }^{2,}{ }^{3}$, L. Coladonato ${ }^{2}$, A. Amati ${ }^{4}$, A. Lia ${ }^{4}$, M. Tampoia ${ }^{5}$, D. Dabbicco ${ }^{6}$, R. Tamma ${ }^{1}$, T. Annese ${ }^{1}$, M. Errede $^{1}$, D. Virgintino ${ }^{(1)}$, D. Ribatti ${ }^{1}$, M. Trojano ${ }^{4}$, F. Iannone ${ }^{2}$, L. Serlenga ${ }^{4}$

${ }^{1}$ Unit of Human Anatomy and Histology, Department of Basic Medical Sciences, Neuroscience and Sense Organs, University of Bari, Italy

${ }^{2}$ Unit of Rheumatology, Department of Emergency and Organ Transplantation, University of Bari, Italy

${ }^{3}$ Service de Physiologie, Unité d'Explorations Fonctionnelles Musculaires, Hôpitaux Universitaires de Strasbourg, France

${ }^{4}$ Unit of Neurophysiopathology, Department of Basic Medical Sciences, Neuroscience and Sense Organs, University of Bari, Italy

${ }^{5}$ Unit of Clinical Pathology, Department of Biomedical Sciences and Human Oncology, University of Bari, Italy

${ }^{6}$ Institute of General Surgery "G. Marinaccio", Department of Emergency and Organ Transplantation, University of Bari

Few data are available concerning the phenotype of macrophages in Idiopathic Inflammatory Myopathies (IIMs). Besides their role in innate immunity, macrophages are also involved in tissue regeneration and angiogenesis promotion.

The density and distribution of the subsets of macrophages, capillary and regenerating myofibres were investigated in human muscle samples from diagnostic biopsies of patients affected by IIMs [6 anti-3hydroxy-3-methylglutaryl coenzyme A reductase (anti-HMGCR) Immune Mediated Necrotizing Myopathy (IMNM), 3 anti-signal recognition particle (anti-SRP) IMNM, 6 seronegative IMNM, 6 adult Dermatomyositis (DM), 6 Polymyositis (PM), 5 PM with mitochondrial pathology (PM-Mito), 6 sporadic Inclusion Body Myositis (sIBM), 6 Scleromyositis, 6 Anti-Synthetase Syndrome with Myositis (ASS), 3 control samples from mitochondrial myopathy (MM), and 6 control samples from age-matched, asymptomatic, hyperCK level individuals.

Endomysial capillary density and capillary/fiber ratio were higher than control group in HMGCR+ IMNM and MM, but lower in DM, PM, Scleromyositis and sIBM.

M1 macrophages were preferentially associated with endomysial microvessels in HMGCR+ IMNM and their density significantly correlated with microvessel density. Most of M1 macrophages expressed Vascular Endothelial Growth Factor AA (VEGF) and Stromal cellDerived Factor 1, which likely promote angiogenesis acting on microvascular cells. Additional VEGF was also immunolocalized in myofibres specifically in HMGCR+ IMNM. Our findings show that a reciprocal cross-talk among myofibres, infiltrating macrophages, and microvascular cells occurs, suggesting a restorative role for macrophages in modulating the muscle regeneration potential and angiogenesis.

Higher muscle damage in patients with anti-Mi2-positive dermatomyositis: a single centre retrospective cohort

F. Girolamo ${ }^{1}$, M. Fornaro ${ }^{2}$, M. Giannini ${ }^{2,}{ }^{3}$, A. Amati ${ }^{4}$, A. $\mathrm{Lia}^{4}, \mathrm{M}$. Tampoia $^{5}$, D. Dabbicco ${ }^{6}$, L. Coladonato ${ }^{2}$, M. Trojano ${ }^{4}$, L. Serlenga ${ }^{4}$, F. Iannone $^{2}$ 
${ }^{1}$ Unit of Human Anatomy and Histology, Department of Basic Medical Sciences, Neuroscience and Sense Organs, University of Bari, Italy

${ }^{2}$ Unit of Rheumatology, Department of Emergency and Organ Transplantation, University of Bari, Italy

${ }^{3}$ Service de Physiologie, Unité d'Explorations Fonctionnelles Musculaires, Hôpitaux Universitaires de Strasbourg, France

${ }^{4}$ Unit of Neurophysiopathology, Department of Basic Medical Sciences, Neuroscience and Sense Organs, University of Bari, Italy

${ }^{5}$ Unit of Clinical Pathology, Department of Biomedical Sciences and Human Oncology, University of Bari, Italy

"Institute of General Surgery "G Marinaccio", Department of Emergency and Organ Transplantation, University of Bari

Dermatomyositis (DM) shows a wide clinical and histopathological spectrum that could differ according to autoantibody status. The aim was to investigate clinical and histopathological hallmarks in our retrospective cohort adult DM patients positive and negative for Myositis Specific Autoantibodies (MSAs).

Clinical data of 56 adult DM patients were collected. Their serum samples were tested using line immunoassays methods for MSAs and Myositis Associated Autoantibodies (MAAs): 14 patients were anti-Mi2+ and 42 anti-Mi2 negative. Histomorphometric quantitative and semiquantitative analyses were carried out on 26 diagnostic muscle biopsy samples (12 anti-Mi2+, 4 anti-MDA+, 1 antiTIF $1 \gamma+, 3$ anti-SAE1/2+, 1 anti-NXP2+, and 5 anti-MSA/MAAnegative).

Anti-Mi2+ DM patients had the highest levels of serum creatine kinase at onset $(4653 \pm 5041 \mathrm{UI} / \mathrm{L}$ vs $809 \pm 1337 \mathrm{UI} / \mathrm{L}, \mathrm{p}<0.001)$ and lower prevalence of interstitial lung disease (ILD) $(2 / 14$ $(14.3 \%)$ vs $16 / 42(38.1 \%), p=0.18)$. Multivariate analysis showed that age at onset (HR 1.07; 95\% CI 1.02-1.13) and rapidly progressive ILD (HR 30.8; 95\% CI 5.6- 169.7) correlated with a poor prognosis in the entire cohort. Higher prevalences of necrotic/ degenerating myofibers with sarcoplasmic deposition of membrane attack complex, of endomysial macrophage number and of perifascicular atrophy score were observed in anti-Mi2+ DM patients.

Our data confirm that anti-Mi2+ patients represent a specific DM subset with higher muscle damage, sarcoplasmic deposition of complement, and endomysial macrophages infiltration as histological hallmarks.

\section{Exon 1 nonsense mutation of dystrophin gene and exception to the reading-frame rule}

A. Govoni ${ }^{1}$, G. Ricci ${ }^{1}$, A. Lo Gerfo ${ }^{1}$, V. Castiglione ${ }^{2}$, L. Cali ${ }^{3}$, F. Baldinotti ${ }^{1}$, A. Rocchi ${ }^{1}$, M. Emdin ${ }^{2}$, G. Siciliano ${ }^{1}$

${ }^{1}$ Neurological Clinics, Department of Clinical and Experimental Medicine, University of Pisa, Italy

${ }^{2}$ Cardiology Division, Fondazione Toscana Gabriele Monasterio, Pisa, Italy

${ }^{3}$ Department of Surgical, Medical, Molecular Pathology and Critical Area, University of Pisa, Pisa, Italy

Mutations in the dystrophin gene are associated with the severe phenotype of Duchenne muscular dystrophy (DMD) when determining the absence of the protein, and with the milder and more variable phenotype of Becker muscular dystrophy (BMD) when determining the synthesis of a protein reduced by size or quantity.

We present the case of a 35-year-old man, a competitive basketball player, suffering from a very mild form of Becker Muscular Dystrophy, manifested with an episode of rhabdomyolysis after a sport competition. Genetic analysis revealed the presence of the point mutation c.12G>A in exon 1 , not previously described, which determines a premature stop signal in the protein (p.Trp4X).
Subsequent cardiological investigations also revealed an initial dilation of the left ventricle, while the qualitative muscle magnetic resonance imaging study did not show pathological findings in the muscles.

Nonsense mutations are notoriously associated with DMD phenotype but when are located in exon 1, they represent an exception to the readingframe rule and cause the milder BMD phenotype. In fact, despite generating a premature stop codon, during translation an alternative start site located further downstream at exon 6 , is recognized and a shorter but functional protein $(60 \mathrm{KDa})$ is synthesized.

\section{Sensorimotor axonal polyneuropathy and a VUS mutation in} Transthyretin gene

F. Gragnani ${ }^{1}$, F. Gilio ${ }^{1}$, R. Iulianella ${ }^{2}$, I.F. Pestalozza ${ }^{1}$, F. Cortese ${ }^{3}$, A.M. Cipriani $^{1}$, E.M. Pennisi ${ }^{3}$

${ }^{1}$ Neurology and Neurophysiopathology Unit, S.Pertini Hospital, ASL Roma2, Italy

${ }^{2}$ ASL Roma2 - S.Pertini Hospital, Cardiology Unit, Italy

${ }^{3}$ ASL Roma1 - San Filippo Neri Hospital, Neurology Unit, Italy

Recently it has been suggested a possible association between presumed nonamyloidogenic mutations in the transthyretin (TTR) gene and the development of autonomic (AN) and small fiber neuropathy (SFN). The authors found in SFN patients (17 of 47 total patients, 36\%) at least 1 point mutation in the TTR gene (variant of unknown significance, VUS) and they found in the subgroup of patients with both SFN and AN (15 of 22;68\%) one or both c76G >A and c337-18G >C VUS mutations (1).

We report the cases of two patients with a sensorimotor axonal polyneuropathy with evidence of c.337-18G $>\mathrm{C}$ mutation in the TTR gene. Both patients (two males, 67 and 68 years old) reported numbness and tingling in the feet from about 2 years; neurological examination showed decreased sensation in the lower extremities, hypoactive deep tendon reflexes, without significant motor and autonomic involvement (mild erectile dysfunction); serologic tests were normal, excluding common causes of polyneuropathies; to date cardiologic tests (ECG, echocardiography) showed no signs of infiltrative cardiomyopathy, however further investigations have been planned in order to assess the presence of amyloid deposits (sural nerve biopsy, bone scintigraphy with DPD) and involvement of other organs (2).

Discussion: our data may contribute to amplify the number of cases possibly correlate to this mutation; however, more studies are needed to evaluate the frequency and possible pathogenetic role of this variant in patients with polyneuropathy of unknown etiology.

1) Levine T.D. et al, Muscle Nerve 2018 Jan;57(1):140-142

2) Adams D. et al., Journal of Neurology. Published online 06.01.2020; https://doi.org/10.1007/s00415-019-09688-0

Recommendations for presymptomatic genetic testing for ATTRv in the era of effective therapy: a multicenter Italian study

M. Grandis ${ }^{1}$, L.Obici ${ }^{2}$, G. Ferrandes ${ }^{3}$, D. Pareyson ${ }^{4}$, C. Briani ${ }^{5}$, C. Danesino $^{2}$, M. Canepa ${ }^{1}$, A. Mazzeo ${ }^{6}$, L. Trevisan ${ }^{1}$, S. Fenu ${ }^{4}$, F. Cappelli $^{7}$, F. Perfetto ${ }^{7}$, L. G. Pradotto ${ }^{8}$, F. Benedicenti ${ }^{9}$, G.M. Fabrizi ${ }^{10}$ G. Bisogni ${ }^{11}$, M. Luigetti ${ }^{12}$, P. Rimessi ${ }^{13}$, L. Melchiorri $^{13}$, G. Tini ${ }^{1}$, C. Gemelli $^{1}$, S. Tozza ${ }^{14}$, D. Severi ${ }^{14}$ and P. Mandich ${ }^{1}$.

${ }^{1}$ University of Genova, Genova, Italy,

${ }^{2}$ Fondazione IRCCS Policlinico San Matteo, Pavia, Italy

${ }^{3}$ IRCCS Ospedale Policlinico San Martino, Genova, Italy

${ }^{4}$ Fondazione I.R.C.C.S. Istituto Neurologico Carlo Besta, Milano, Italy

${ }^{5}$ University of Padova, Padova, Italy

${ }^{6}$ University of Messina, Messina, Italy

${ }^{7}$ Azienda Ospedaliera Unversitaria Careggi, Firenze, Italy 
${ }^{8}$ Istituto Auxologico Italiano Piancavallo, Oggebbio (Verbania) Italy

${ }^{9}$ Azienda Sanitaria dell'Alto Adige, Bolzano, Italy

${ }^{10}$ University of Verona, Verona, Italy

${ }^{11}$ Centro Clinico Nemo Adulti-Fondazione Policlinico Universitario Agostino Gemelli IRCCS, Roma, Italy

${ }^{12}$ Fondazione Policlinico Universitario Agostino Gemelli IRCCS, Roma, Italy

${ }^{13}$ Azienda Ospedaliero Universitaria di Ferrara, Ferrara, Italy

${ }^{14}$ University of Naples Federico II, Napoli, Italy

Hereditary transthyretin amyloidosis (ATTRv) is an AD inherited, fatal disease caused by the deposition of amyloid fibrils. In most patients, the clinical onset is around the sixth decade of life. For other late onset fatal diseases, as Huntington disease, protocols for presymptomatic genetic testing (PST) are available.

After an initial survey to collect the different protocols on PST, two roundtable expert meetings were attended by 24 ATTRv experts, covering all the Italian regions, to identify points of discussion and achieve a final consensus on PST procedures, tailored to the Italian population. By November 2019, the Italian centers followed 497 families affected by ATTRv with 209 presymptomatic carriers. The average age at PST was 40.5 years of age, regardless the specific mutation identified. Half of the Italian centers have a multidisciplinary team, including a neurologist, an internist, a cardiologist, a geneticist and a psychologist, even if in most cases not all the specialists are available. We agreed that the number of clinical evaluations should consist of a pre-test visit, the test-visit and the delivery of the results, thus allowing an informed choice. We all shared that our health system can provide the test and that this option should be preferred to commercial alternatives.

Nowadays, the therapeutic scenario for ATTRv has completely changed with the emerging of novel molecular therapies. Therefore, the protocol for PST should be redefined to offer to potential carriers the possibility to be diagnosed and monitored to detect the initial phases of the disease and start specific treatments.

Long-term Prognosis in Guillain-Barré Syndrome and Clinical Variants: Focus on Motor and Sensory Outcome

S. Grisanti ${ }^{1,2}$, C. Demichelis ${ }^{3}$, A. Zuppa ${ }^{1,2}$, V. Prada $^{1}$, A. Beronio ${ }^{4}$, V. Dorindo $^{1}$, A. Schenone ${ }^{1,2}$, L. Benedetti ${ }^{1,2}$

${ }^{1}$ Department of Neuroscience, Rehabilitation, Ophthalmology, Genetics, Maternal and Child Health (DiNOGMI), University of Genova, Italy

${ }^{2}$ IRCCS, Ospedale Policlinico San Martino, Genova, Italy

${ }^{3}$ S.O.C. Neurologia, Azienda Ospedaliera Santi Antonio e Biagio e Cesare Arrigo, Alessandria, Italy

${ }^{4}$ U.O Neurologia, Ospedale Sant'Andrea, La Spezia, Italy

Introduction: Many data have been published in literature about clinical presentation, electrodiagnostic studies and therapy in Guillain-Barré Syndrome (GBS), but less is known about the long-term prognosis. Herein, we evaluate the long-term prognosis in a wide group of GBS patients.

Methods: The analysis was conducted prospectively in $70 \%$ of patients and retrospectively in $30 \%$ of them. Epidemiological, clinical, electrophysiological and therapeutic data were collected. Outcomes were measured in terms of mortality, gait recovery, strength and disability scales (i.e. GBS-DS, MRC-SS).

Results: We included 107 patients admitted from 2003 to 2018, 87 with classic subtypes of GBS (AIDP/AMAN/AMSAN) and 20 with clinical variants (Miller-Fisher Syndrome, Pharyngeal-cervical-brachial weakness, Isolated facial diplegia with distal paraesthesia and Bickerstaff's brainstem encephalitis). Patients with a follow-up shorter than six months were excluded. The mean follow-up was 3.39 years ( 6 months-14 years). Mortality was $16 \%$ and only among patients with classic GBS. $26 \%$ of patients didn't recover a normal gait; only $38 \%$ of patients had a complete recovery. About $30 \%$ of patients had persistent distal muscle weakness, paraesthesia and alterations of the emotional state (anxiety/depression) even after years from the onset. Respiratory failure and dysautonomia correlated with a worse outcome in terms of GBS-DS and MRC-SS. A better outcome correlated with a lower disability at the baseline and early treatment (within 7 days from the onset). Among clinical variants there were no deaths.

Conclusions: A worse outcome is related to respiratory failure, dysautonomia, axonal forms and later treatment. Among GBS variants, MFS is confirmed the most benign form, while, surprisingly, $50 \%$ of Pharyngeal-cervical-brachial variant and $100 \%$ of Isolated facial diplegia patients had significant persistent upper limb or facial weakness respectively.

Virtual genetic counselling in neuromuscular diseases:a pilot telegenetics project

F. Gualandi ${ }^{1}$, M. Neri ${ }^{1}$, M. Farnè ${ }^{1}$, F. Fortunato ${ }^{1}$, G. Vita ${ }^{2}$, M. Pane ${ }^{3}$, V. Sansone $^{4}$, A. Barp ${ }^{4}$, E. Albamonte ${ }^{4}$,T. Evangelista ${ }^{5}$, A. Ferlini ${ }^{1}$

${ }^{1}$ Unit of Medical Genetics, Department of Medical Sciences, University of Ferrara

${ }^{2}$ NEMO Clinical Center in Messina (NEMO Sud), Messina, Italy.

${ }^{3}$ NEMO Clinical Center in Rome, Rome, Italy.

${ }^{4}$ NEMO Clinical Center in Milan, Milan, Italy

${ }^{5}$ Institut de Myologie, Groupe Hospitalier Pitié-Salpêtrière, Paris, France

Telemedicine is defined by the European Commission as "the provision of health care services, through the use of information and communication technology (ICT), in situations where the health professional and the patient are not in the same location". It is increasingly emerging as an innovative approach to facilitate diagnosis and treatment of several conditions, included rare or complex diseases. Developing telemedicine technologies is within the mission of European Reference Networks, including ERN on rare neuromuscular disorders (ERN EURO-NMD), which represent a new and unique example of a high-level e-health-based systems. "Telegenetics" is an application of telemedicine for the delivery of genetic counseling and testing at a distance: the non-homogeneous availability of genetic services among countries and the increasing demand of high level expertise, make telegenetics an attractive alternative to traditional on-site genetic counseling. As a pilot experience in Italy, our group is developing and testing a model for "Virtual Medical Genetics Clinics" based on ICT, in collaboration with NEMO centers and a Cardiology Unit in Fano (PU), to provide genetic telecounselling in hereditary neuromuscular and cardiac diseases. Audiovisual telecommunications technology and a dedicated platform ("Lepida WebConference") is used to provide "real time" interaction between patients and counsellors. Aim of this project, called "TeleNEwCARe" (for Telegenetics in Neuromuscular and Cardiac diseases) and already approved by the local Ethic Committee for adherence to privacy statements, is to evaluate the application of telegenetics as alternative to traditional counseling, users experience (through dedicated questionnaires) and to assess existing barriers to IT tools application and implementation.

\section{Efgartigimod in Autoimmune Neuromuscular Diseases}

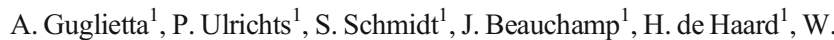
Parys $^{1}$

${ }^{l} \operatorname{argenx} B V$

Zwinjaarde, Belgium 
Introduction: Myasthenia gravis (MG) and chronic inflammatory demyelinating polyneuropathy (CIDP) are rare autoimmune diseases, mediated by pathogenic immunoglobulin $\mathrm{G}(\mathrm{IgG})$ autoantibodies that result in debilitating and potentially life-threatening consequences.

Neonatal Fc receptor $(\mathrm{FcRn})$ is central to $\mathrm{IgG}$ homeostasis and is responsible for the long half-life of IgG including pathogenic autoantibodies. Efgartigimod is a human IgG1 antibody Fc-fragment, a natural ligand of FcRn, engineered for increased, pH-dependent, FcRn affinity. Efgartigimod outcompetes endogenous IgG binding, preventing recycling, and as a consequence increased $\mathrm{IgG}$ degradation.

Here we update the findings of recent studies.

Methods: Efgartigimod $\geq 10 \mathrm{mg} / \mathrm{kg}$ IV has been administered to $>120$ subjects, in healthy volunteers and three autoimmune disease studies.

Results: Efgartigimod, reduced all IgG subtypes, but did not affect other immunoglobulins or albumin. No safety concerns have been identified. In a Phase 2 MG study in which patients $(n=24)$ received 4 weekly infusions, clinically meaningful improvements across four MG scales occurred. $75 \%$ efgartigimod patients achieved $\geq 2$-point $\mathrm{MG}$-ADL reduction for $\geq 6$ consecutive-weeks versus $25 \%$ placebo $(p=0.039$ ). At end of study, 8 weeks after last dose, 6/12 efgartigimod patients maintained clinically meaningful improvement of MG-ADL score, suggesting sustained effect beyond $\mathrm{IgG}$ reduction.

Conclusion: FcRn plays a critical role in IgG regulation. Efgartigimod was well tolerated, reduced IgG and AChR-antibodies, and improvement in gMG symptoms was sustained to end of study. A global phase 3 study is ongoing, with additional studies in CIDP, in immune thrombocytopenia (ITP) as well as in pemphigus vulgaris (PV).

\section{Mutations in Supervillin cause myopathy with myofibrillar disorga- nization and autophagic vacuoles}

C. Hedberg-Oldfors ${ }^{1}$, R. Meyer ${ }^{2}$, K. Nolte ${ }^{2}$, Y. Abdul Rahim ${ }^{1}$, Christopher Lindberg ${ }^{3}$, K. Karason ${ }^{3}$, I. Johanne Thuestad ${ }^{4}$, K. Visuttijai $^{1}$, M. Geijer ${ }^{3}$, M. Begemann ${ }^{2}$, F. Kraft ${ }^{2}$, E. Lausberg ${ }^{2}$, L. $\mathrm{Hitpa}^{2}$, R. Götzl ${ }^{2}$, E. J. Luna ${ }^{5}$, H. Lochmüller ${ }^{6}$, S. Koschmieder ${ }^{2}, \mathrm{M}$. Gramlich $^{2}$, B. Gess ${ }^{2}$, M. Elbracht ${ }^{2}$, J. Weis ${ }^{2}$, I. Kurth ${ }^{2}$, A. Oldfors ${ }^{1}$, C. $\mathrm{Knopp}^{2}$

${ }^{1}$ Department of Pathology and Genetics, Sahlgrenska Academy, University of Gothenburg, Gothenburg, Sweden,

${ }^{2}$ RWTH Aachen University, Aachen, Germany,

${ }^{3}$ Sahlgrenska University Hospital, Gothenburg, Sweden,

${ }^{4}$ Skane University Hospital, Malmo, Sweden,

${ }^{5}$ University of Massachusetts Medical School, Worcester, United States,

${ }^{6}$ The Ottawa Hospital; and Brain and Mind Research Institute, University of Ottawa, Ottawa, Canada

We have identified four patients with a childhood/adolescence onset of a myopathy from two unrelated consanguineous families. The patients have wide neck, anteverted shoulders and prominent trapezius muscles together with variable contractures as characteristic features. All patients showed increased levels of serum creatine kinase but no or minor muscle weakness. Mild cardiac manifestations were observed. Light and electron microscopical investigations revealed a structural myopathy with numerous lobulated muscle fibers and considerable myofibrillar alterations with a coarse and irregular intermyofibrillar network. Autophagic vacuoles, as well as frequent and extensive deposits of lipoproteins, including immature lipofuscin were observed. Several sarcolemma-associated proteins such as dystrophin and sarcoglycans were partially mislocalized. Genetic analysis revealed homozygous loss-of-function mutations in SVIL and muscle biopsies showed complete loss of large supervillin isoforms in muscle fibers by western blot and immunohistochemical analyses.

The muscle specific isoform of the supervillin protein, also called archvillin, encoded by the SVIL gene, is a large sarcolemmal myosin IIand F-actin-binding protein. Archvillin binds and co-localizes with costameric dystrophin and binds nebulin, potentially attaching the sarcolemma to myofibrillar Z-lines. Despite its important role in muscle cell physiology suggested by various in vitro studies, there are so far no reports of any human disease caused by SVIL mutations.

The results demonstrate the importance of the supervillin/archvillin protein for the structural integrity of muscle fibers in humans and show that recessive loss-of-function mutations in SVIL cause a distinctive and novel myopathy.

\section{Neuromuscular sarcoidosis: single center experience in 209 consecu-} tive patients

B. Labella ${ }^{1}$, F. Cinetto ${ }^{2,}{ }^{3}$, R. Scarpa ${ }^{2,3}$, R. Manara ${ }^{4}$, E. Pegoraro ${ }^{1}$, C. Agostini $^{2,3}$, C. Briani ${ }^{1}$.

${ }^{1}$ Department of Neurosciences, Neurology Unit, University of Padova, Italy;

${ }^{2}$ Department of Medicine, University of Padova, Italy,

${ }^{3}$ Ca' Foncello Hospital, AULSS 2 Treviso, Italy;

${ }^{4}$ Radiology, University of Padova, Italy.

Sarcoidosis is a systemic inflammatory disease of unknown etiology characterized by non-necrotizing epithelioid granuloma that involves mainly skin, lungs and lymph nodes. Neuromuscular involvement is a rare manifestation of sarcoidosis.

We performed a retrospective study on 209 patients with sarcoidosis followed at the Centre for Rare Diseases of the Treviso and Padua Hospital since 1998, in order to assess the prevalence of neuromuscular manifestations. In our sample, 25/209 (12\%) patients had neuromuscular involvement. Extra-neurological involvement of sarcoidosis allowed us to obtain histopathological confirmation of the disease in most of them (17/25). Cranial neuropathy was a frequent manifestation in agreement with literature data (28\%): 5 facial nerve palsy, 2 optic neuritis and 2 eight nerve involvements. Only one patient showed multiple cranial neuropathy (VII and VIII cranial nerves). Muscular involvement was detected in 2 patients $(1 \%)$. Patients with muscular involvement presented with subacute/ chronic disease onset. Mostly patients with cranial neuropathy (4/7) presented with acute disease onset. Cerebrospinal fluid analysis resulted abnormal in all but one case, showing common but nonspecific inflammation signs. All the patients with cranial neuropathy underwent brain magnetic resonance (MRI) with gadolinium and 3/7 also 18-fluorodeoxyglucose positron emission tomography (18FDG-PET). Of the 2 patients with myopathy, one performed 18FDG-PET in a context of multi-organ involvement, while the other performed electromiography, 18FDG-PET, muscle MRI and positive muscle biopsy. The diagnosis of neuromuscular involvement in sarcoidosis is problematic and imaging studies may have a role, although the biopsy of neural/muscle tissue is still the gold standard in the diagnostic workup.

Cognitive and personality involvment in DM1 patients: is there an age at onset related effect?

\section{E. Lai, G. Spadoni, C. Simoncini, G. Ricci, G. Siciliano}

Clinica Neurologica - Università di Pisa. Università di Pisa, Pisa, Italy

Background: Myotonic Dystrophy type 1 (DM1) is a multisistemic disease in which involvement of the central nervous system is a relevant cause of cognitive and behavioral problems. In addition to confirming the cognitive deficits already reported in the literature, the aim of this study was to investigate the abnormal personality traits in DM1 
Materials and Method: We enrolled 18 patients (Male=12; mean age $=47,56 \pm 14,37)$, separated in 4 subgroups based on age at onset: 1 ) adult ( $\mathrm{n}=6)$ (age: $M=44,83 \pm 9,45), 2)$ late $(\mathrm{n}=7)($ age: $\mathrm{M}=61,43 \pm 3,69), 3)$ juvenile $(n=3)$ (age:M=27,67 $\pm 6,03)$ and 4) childhood $(n=2)($ age: $M=35$ $\pm 4,24$ ). Each patient underwent an evaluation of personality (DSM-5), and a neuropsychological assessment composed by: memory, language and executive tools.

Results and conclusions: neuropsychological investigations show a general impairment in executive functioning $[n=8 / 18(44,44 \%)]$, more frequent for the working memory $[\mathrm{n}=6 / 18(36,11 \%)]$ with a different distribution depending upon age at onset subgroups, where the late onset subgroup has the highest rate $[n=4 / 7(57,14 \%)]$ of compromised scores in auditory-verbal working memory subtest. Episodic memory was also involved, in late $[n=3 / 7(42,86 \%)]$ and in adult subgroups $[n=3 / 6(50 \%)]$. Personality evaluation mainly indicates the presence of obsessivecompulsive traits $[\mathrm{n}=12 / 13(92,31 \%)]$ both in the adult and late onset subgroups, while paranoid traits were the preminent ones also in the juvenile subgroup $[\mathrm{n}=8 / 16(50 \%)]$. These results suggest how different cognitive and personality profiles can characterize DM1 upon its age at onset and therefore severity of the disease, encouraging future investigation to further characterize the clinical burden of CNS involvement and the value of its inclusion as primary outcome measure for clinical trials in this disease.

Intraepidermal nerve fiber density as a biomarker of disease severity in hereditary transthyretin amyloidosis with polyneuropathy: data from an Italian cohort

L. Leonardi ${ }^{1}$, A. Truini ${ }^{2}$, A. Fasolino ${ }^{2}$, E. Galosi ${ }^{2}$, M. Luigetti ${ }^{3}$, L.

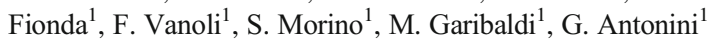

${ }^{1}$ Neuroscience, Menatal Health and Sensory Organs Departement (NESMOS), Sapienza University, Rome, Italy

${ }^{2}$ Departement of Human Neuroscience, Sapienza, University, Rome, Italy

${ }^{3}$ IRCCS Policlinico A. Gemelli, Rome, Italy

Introduction: Hereditary transthyretin amyloidosis with polyneuropathy $($ ATTRv-PN) is a multysystemic inherited disease in which nerve involvement is predominant. With the availability of effective treatments for this potentially fatal disease, a biomarker of disease severity and progression is highly demanded. Herein, we evaluate the relationship of skin biopsy with clinical and paraclinical variable.

Methods: We evaluated 26 subjects with amyloidogenic mutations in TTR (9 asymptomatic, 17 symptomatic; 16 Val30Met, 10 NonVal30Met) performing neurological examination, NIS, small fiber neuropathy symptoms inventory questionnaire (SFN-SIQ), NCS, skin biopsy [intraepidermal nerve fiber density (IENFD)]. Patients were stratified according to a modified version of peripheral neuropathy disability score (mPND) where 1a represents subjects with normal NCS and IENFD reduction and $1 \mathrm{~b}$ represents subjects with sural nerve SAP amplitude reduction. Statistical analysis was performed using non-parametric tests. Results: We found an IENFD reduction in 18\% (2/9) of asymptomatic subjects vs $100 \%$ (17/17) of symptomatic subjects. Mean IENFD was $12.3+/-3.6$ in asymptomatic subjects vs $3.4+/-2.9$ in symptomatic subjects $(\mathrm{p}=0.0003)$. IENFD was deeply reduced in severely affected patients $(\mathrm{mPND}>2)$. No statistically significant difference was found between Val30Met and Non-Val30Met IENFD. IENFD correlated with age at biopsy $(\mathrm{r}=-0.6, \mathrm{p}=0.001)$, disease duration $(\mathrm{r}=-0.5, \mathrm{p}=0.02), \mathrm{mPND}$ $(\mathrm{r}=0.8, \mathrm{p}<0.0001)$, NIS $(\mathrm{r}=-0.8, \mathrm{p}<0.0001)$, SFN-SIQ $(\mathrm{r}=-0.5, \mathrm{p}=0.008)$, sural SAP amplitude $(r=-0.7, p<0.0001)$.

Conclusions: Correlating with NIS and mPND, IENFD could represent a reliable biomarker of disease severity in ATTRv-PN. Moreover, small ber neuropathy symptoms measured with (SNF-SIQ) correlated with IENFD, showing accordance between clinical and pathological definition of SFN in ATTRv-PN.
A case of COVID19 associated pharyngeal-cervical-brachial variant of Guillain-Barrè Syndrome

G. Liberatore, ${ }^{1}$ T. De Santis, ${ }^{2}$ P. E. Doneddu, ${ }^{1}$ F. Gentile, ${ }^{1}$ A. Albanese, ${ }^{2}$ ${ }^{3}$ E. Nobile-Orazio, 1,4

${ }^{1}$ Neuromuscular and Neuroimmunology Service, IRCCS Humanitas Clinical and Research Institute, Rozzano, Milan, Italy

${ }^{2}$ Department of Neurology, IRCCS Humanitas Clinical and Research Institute, Rozzano, Milan, Italy

${ }^{3}$ Institute of Neurology, Catholic University of the Sacred Heart, Milan, Italy

${ }^{4}$ Department of Medical Biotechnology and Translational Medicine, Milan University, Milan, Italy

Objective: to report the first case of Pharyngeal-Cervical-Brachial (PCB) variant of Guillain Barrè Syndrome (GBS) associated with severe acute respiratory coronavirus 2 (SARS-COV-2) infection.

Methods: Patient data were obtained from clinical records from Humanitas Clinical and Research Institute, Rozzano, Italy.

Results: A 49-year-old man presented with fever and cough for a week and, after chest CT scan e nasopharyngeal swab positive for SARS-CoV-2, was diagnosed with coronavirus disease 19 (COVID-19). Few days after admission, his clinical condition deteriorated requiring mechanical ventilation. After weaning from mechanical ventilation after 23 days, clinicians reported dysphagia, VII and XII cranial nerve impairment, neck flexor and upper limbs hyposthenia with minimal involvement of lower limbs, no sensory deficit, dysautonomia with tachycardia. CSF showed mild hyperproteinorrachia, neurophysiological exams highlighted signs of axonal neuropathy. Patient was not treated with a specific immunotherapy, since the symptoms appeared to improve spontaneously with a good recovery.

Conclusions: this is the first report of the rare PCB variant of GBS linked to COVID-19. The possible mechanism underlying this syndrome could be an aberrant immune response more than a direct viral damage. More data are needed to clarify the exact pathophysiology, but our case warns clinician to be aware of the possible manifestation of rare neurological syndromes after COVID-19 infection.

Distinguishing features of Acute- and Chronic-Onset Chronic Inflammatory Demyelinating Polyradiculoneuropathy

G. Liberatore, ${ }^{1}$ F. Manganelli, ${ }^{2}$ D. Cocito, ${ }^{3}$ R. Fazio, ${ }^{4}$ C. Briani, ${ }^{5}$ M. Filosto, ${ }^{6}$ L. Benedetti, ${ }^{7}{ }^{8}$ G. Antonini, ${ }^{9}$ G. Cosentino, ${ }^{10}$ S. Jann, ${ }^{11}$ A. Mazzeo, ${ }^{12}$ A. Cortese, ${ }^{13,}{ }^{14}$ G. A. Marfia, ${ }^{15}$ A. M. Clerici, ${ }^{16}$ G. Siciliano, ${ }^{17}$ M. Carpo, ${ }^{18}$ M. Luigetti, ${ }^{19}$ G. Lauria, ${ }^{20,}{ }^{21}$ T. Rosso, ${ }^{22} \mathrm{G}$. Cavaletti, ${ }^{23}$ P. E. Doneddu, ${ }^{1}$ L. Santoro, ${ }^{2}$ E. Peci, ${ }^{3}$ S. Tronci, ${ }^{4}$ M. Ruiz, ${ }^{5}$ S. Cotti Piccinelli, ${ }^{6}$ A. Schenone, ${ }^{7}$ L. Leonardi, ${ }^{9}$ A. Toscano, ${ }^{12}$ L. Piccolo, ${ }^{13}$ G. Mataluni, ${ }^{14}$ E. Nobile-Orazio, ${ }^{1,24}$ on the behalf of the Italian CIDP Database Study Group.

${ }^{1}$ Neuromuscular and Neuroimmunology Service, Humanitas Clinical and Research Institute, Rozzano, Milan, Italy

${ }_{2}$ Department of Neuroscience, Reproductive Sciences and Odontostomatology, University of Naples 'Federico II', Naples, Italy

${ }^{3}$ Presidio Sanitario Major, Istituti Clinici Scientifici Maugeri, Turin, Italy

${ }^{4}$ Division of Neuroscience, Department of Neurology, Institute of Experimental Neurology (INSPE), San Raffaele Scientific Institute, Milan, Italy

${ }^{5}$ Neurology Unit, Department of Neuroscience, University of Padova, Padova, Italy

${ }^{6}$ Center for Neuromuscular Diseases and Neuropathies, Unit of Neurology, ASST 'Spedali Civili', University of Brescia, Brescia, Italys ${ }^{7}$ Department of Neuroscience, Rehabilitation, Ophthalmology, Genetics, Maternal and Child Health, University of Genoa and IRCCS AOU San Martino-IST, Genoa, Italy 
${ }^{8}$ Neurology Unit, Sant'Andrea Hospital, La Spezia, Italy

${ }^{9}$ Unit of Neuromuscular Diseases, Department of Neurology Mental Health and Sensory Organs (NESMOS), Faculty of Medicine and Psychology, 'Sapienza' University of Rome, Sant'Andrea Hospital, Rome, Italy

${ }^{10}$ Department of Experimental BioMedicine and Clinical Neurosciences (BioNeC), University of Palermo, Palermo, Italy

${ }^{11}$ Department of Neuroscience, Niguarda Ca' Granda Hospital, Milan, Italy

12 Department of Clinical and Experimental Medicine, Unit of Neurology, University of Messina, Messina, Italy

${ }^{13}$ IRCCS Foundation C. Mondino National Neurological Institute, Pavia, Italy

${ }^{14}$ Molecular Neurosciences, University College London, London, UK

${ }^{15}$ Dysimmune Neuropathies Unit, Department of Systems Medicine, Tor Vergata University of Rome, Rome, Italy

${ }^{16}$ Neurology Unit, Circolo \& Macchi Foundation Hospital, Insubria

University, DBSV, Varese, Italy

${ }^{17}$ Neurology Unit, Department of Clinical and Experimental Medicine, University of Pisa, Pisa, Italy

${ }^{18}$ Neurology Unit, ASST Bergamo Ovest-Ospedale Treviglio, Treviglio, Italy

${ }_{19}$ Fondazione Policlinico Universitario A. Gemelli IRCCS, UOC Neurologia, Università Cattolica del Sacro Cuore, Rome, Italy.

${ }^{20}$ Unit of Neuroalgology, IRCCS Foundation 'Carlo Besta' Neurological Institute, Milan, Italy

${ }^{21}$ Department of Biomedical and Clinical Sciences 'Luigi Sacco', University of Milan, Milan, Italy

${ }^{22}$ ULSS2 Marca Trevigiana, UOC Neurologia-Castelfranco Veneto, Treviso, Italy

${ }^{23}$ School of Medicine and Surgery and Experimental Neurology Unit, University of Milano-Bicocca, Monza, Italy

${ }^{24}$ Department of Medical Biotechnology and Translational Medicine, Milan University, Milan, Italy

Objective: Chronic Inflammatory Demyelinating Polyradiculoneuropathy (CIDP) can have heterogeneous presentations with up to $16 \%$ of the patients having an acute onset (A-CIDP) similar to Guillain-Barré Syndrome (GBS). Little is known abuot the clinical, laboratory and neurophysiologic features distinguishing patients with A-CIDP from those with a chroniconset (C-CIDP).

Methods: We implemented a multicentric web-based database to collect data from patients with CIDP followed throughout Italy. We defined ACIDP a neuropathy initially diagnosed as GBS that continued to progress or relapsed after more than two months from disease onset.

Results: By February 2020, we included 437 patients with a confirmed diagnosis of typical CIDP or variants. Forty-two patients (10\%) had ACIDP, followed by a relapsing or progressive course in $68 \%$ and $32 \%$ of cases (55\% and $45 \%$ in typical CIDP). Compared to patients with C-CIDP, patients with A-CIDP were younger at onset ( 45.2 vs 50.3 versus, $\mathrm{p}=0.017$ ), had a worse INCAT score at enrollment ( 3.2 vs $2.5, p=0.048$ ), a more frequent involvement of strength ( $100 \%$ vs $90 \%, \mathrm{p}=0.014)$ and cranial nerve $(50 \%$ vs $17 \%, \mathrm{p}<0.001)$. A-CIDP patients more frequently had increased CSF proteins ( $91 \%$ vs $65 \%, \mathrm{p}=0.019)$ with a higher median value ( 111 vs 88 $\mathrm{mg} / \mathrm{dL}$ ), while there was no significant difference on electrodiagnostic studies. A-CIDP patients had a more frequent overall response to therapy compared to C-CIDP ( $97 \%$ vs $82 \%, \mathrm{p}=0.027$ ) and increased titers of antibodies to $\mathrm{CNTN}-1$ or NF-155 ( $15 \%$ vs $3.5 \%, \mathrm{p}=0.029)$.

Discussion: Some distinguishing features are present in A-CIDP patients and may reflect some overlap between CIDP and GBS.

\section{Myotonic Dystrophy type 2 unmasked by physical exercise following COVID-19 lockdown}

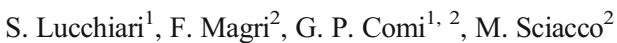

${ }^{1}$ Dino Ferrari Center, Department of Pathophysiology and Transplantation, University of Milan, Milan, Italy

${ }^{2}$ Neuromuscular and Rare Disease Unit, Department of Neuroscience, IRCCS Foundation Ca' Granda Ospedale Maggiore Policlinico, University of Milan, Milan, Italy.

Myotonic dystrophy type 2 (DM2) is a dominantly inherited syndrome, also known as PROMM, proximal myotonic myopathy, since it causes proximal skeletal muscle weakness, myalgia, and variable degree of myotonia. We describe a 25 years old woman affected by a DM2 form which became manifested when returning to physical exercise after Covid-19related lockdown. The patient assessed the Emergency Room of our hospital referring the onset of severe and diffuse muscle cramps and myalgia forcing her to use a wheelchair - after the first $7 \mathrm{Km}$-long walk she took after the lockdown. She used to walk up to $10 \mathrm{Km}$ before without any problems. She further referred that in the last few months she had noted difficulties in opening her hands after prolonged contraction. At neurological examination, she presented generalized muscle weakness more evident in the lower limb-girdle and only partially pain-related. Myotonia was evident in both hands and foot fingers and was confirmed at electromyography. Serum CK levels in the ER were over 47,000 U/L and went back to normal in few months along with muscle strength. Regarding medical history, she underwent cataract surgery at the age of 20 years, and, recently, she had been taking hormones for amenorrhea. Family history was negative. DM2 genetic assay revealed a pathological expansion in intron 1 of $C N B P$ gene. Physical exercise is considered beneficial for a correct management of myotonic disorders, however, the effects of its suspension for a long period might be detrimental and, in this case, resulted in massive rhabdomyolysis.

Inotersen to treat hATTR polyneuropathy: tolerability and management from two centre experience

M. Luigetti MD ${ }^{1,2}$, A. Romano ${ }^{2,3}$, A. Mazzeo ${ }^{4}$, A. Di Paolantonio ${ }^{2}, \mathrm{G}$. Bisogni $^{2}$, V. Guglielmino ${ }^{1,2}$, M. Russo ${ }^{4}$, L. Gentile ${ }^{4}$, A. Toscano ${ }^{4}$, G. Vita $^{4}$, M. Sabatelli' ${ }^{2,3}$.

${ }^{I}$ Fondazione Policlinico A. Gemelli IRCCS. UOC Neurologia, Italy

${ }^{2}$ Università Cattolica del Sacro Cuore. Sede di Roma, Italy

${ }^{3}$ Centro Clinico NEMO Adulti Roma, Italy

${ }^{4}$ Unit of Neurology and Neuromuscular Diseases, Department of Clinical and Experimental Medicine, University of Messina, Italy.

Introduction: Hereditary transthyretin amyloidosis (hATTR) is an underdiagnosed disease commonly associated to several TTR mutations, producing an instable protein that deposits in different tissues (nerve, myocardium, kidney and vitreum). Inotersen, an antisense oligonucleotide inhibitor, was recently approved in the USA and Europe for the treatment of hATTR polyneuropathy based on the positive results of NEURO-TTR trial.

Methods: We treated first patient in August 2015 (284 mg subcutaneously, weekly). Since then, other ten patients started inotersen treatment. At that time, two patients were assuming diflunisal and nine tafamidis. Nowadays, only one of them is in double therapy with tafamidis. For each patient, we periodically evaluated NIS scale, QOL-DN and Sudoscan. We regularly checked blood tests, too.

Results: Age at first administration varied between 50 and 85 years. Five patients had the Phe64Leu mutation, three the Val30Met, two the Glu89Gln and one the Thr59Lys. We observed for all patients a stabilization of clinical condition. However, we had to modify drug dosage in four patients because of the platelets count lowering. Furthermore, one patient temporarily discontinued because of creatinine clearance reduction, without evidence of glomerulonephritis. Two patient experienced a 
serious adverse event resulting in death: one for complication after lung surgery (due to severe pleural effusion) and one probably for sudden cardiac death. In both cases, it's highly unlikely that these episodes are related to inotersen administration.

Conclusion: Inotersen is well tolerated, with a manageable safety profile through regular blood tests monitoring and has been shown to stabilize neuropathy progression.

\section{Muscle MRI pattern recognition: a sample study using a MRI based tool}

Maffei $\mathrm{M}^{1}$, Giannotta $\mathrm{M}^{2}$, Scarpini $\mathrm{G}^{2}$, Cirignotta $\mathrm{L}^{1}$, Pini $\mathrm{A}^{2}$

${ }^{1}$ UO Neuroradiologia IRCCS Istituto delle Scienze Neurologiche di Bologna, Ospedale Bellaria, Italy

${ }^{2}$ UO Neuropsichiatria Infantile Neuromuscular Pediatric Centre, IRCCS Istituto delle Scienze Neurologiche di Bologna, Ospedale Bellaria, Italy

Muscle MRI contributeto the genetic and clinical characterization of neuromuscular diseases (NMD)(Khana V 2014). Even if the recognition of specific muscle patterns is still limited, the development of dedicated software-based tools is encouraged (Verdú-Díaz J 2020). Furthermore, many patients do not have a precise genetic definition and combining their patterns with the known ones can be useful. Finally muscle MRI patterns have recently proved useful for better understand natural hystoryas for SMA (Brogna C, 2019). In order to facilitate the diagnosis and categorize atypical cases as best as possible, we have built a database of genetically NMD with a known MRI pattern. Subsequently, template maching profileswere obtained, corresponding to the various known genetic forms. The data of 132 muscle MRI of patients referring to the Neuromuscular Pediatric Center of the ISNB were then entered and the index of deviation from the known patterns was identified. Patients were genetically determined (49) or not (64) and 19 muscle MRI represented a second exam after at least 1 year. A good correlation has emerged within the forms RYR1, SEPN1, COLVI and Duchenne. In SMA, evidence of distal involvement was confirmed, stable after at least 1 year of treatment with Nusinersen. In the non genetically determined forms the most frequently found pattern was that of the anterior proximal compartment involvement. On the basis of these preliminary results, we are developing a software-based tool that will help to classify the atypical cases according to the models and to better interpret the genetic results of NGS.

\section{Nusinersen safety and effects on motor function in adult spinal mus- cular atrophy type 2 and 3}

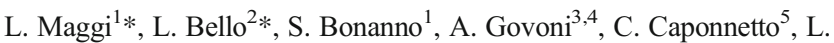
Passamano $^{6}$, M. Grandis ${ }^{5,7}$, F. Trojsi ${ }^{8}$, F. Cerri ${ }^{9}$, M. Ferraro ${ }^{10}$, V. Bozzoni $^{2}$, L. Caumo ${ }^{2}$, R. Piras ${ }^{11}$, R. Tanel ${ }^{12}$, E. Saccani ${ }^{13}$, M. Meneri ${ }^{3}$, V. Vacchiano ${ }^{14}$, G. Ricci ${ }^{4}$, G. Sorarù ${ }^{2}$, E. D’Errico ${ }^{15}$, I. Tramacere ${ }^{16}$, S. Bortolani $^{10}$, G. Pavesi ${ }^{17}$, R. Zanin ${ }^{18}$, PT, M Silvestrini ${ }^{19,20}$, L. Politano ${ }^{6}$, A. Schenone ${ }^{5,7}$, S.C. Previtali ${ }^{9}$, A. Berardinelli ${ }^{21}$, M. Turri ${ }^{22}$, L. Verriello $^{23}$, M. Coccia $^{19}$, R. Mantegazza ${ }^{1}$, R. Liguori ${ }^{14}$, M. Filosto ${ }^{24}$, G. Marrosu ${ }^{25}$, G. Siciliano ${ }^{4}$, I.L. Simone ${ }^{15}$, T. Mongini ${ }^{10}$, G. P. Comi $^{3,26 \#}$, E. Pegoraro ${ }^{2 \#}$.

*These authors contributed equally

\# Both senior authors contributed equally

${ }^{1}$ Neuroimmunology and Neuromuscular Disease Unit, Fondazione IRCCS Istituto Neurologico Carlo Besta, Milano, Italy.

${ }^{2}$ Myology Institute, Department of Neuroscience, University of Padova, Italy.

${ }^{3}$ Neuromuscular and Rare Disease Unit, Fondazione IRCCS Ca' Granda Ospedale Maggiore Policlinico, Milano, Italy;
${ }^{4}$ Department of Clinical and Experimental Medicine, University of Pisa, Italy.

${ }^{5}$ IRCCS Ospedale Policlinico San Martino, Genova, Italy.

${ }^{6}$ Cardiomyology and Medical Genetics Unit, University Hospital "L. Vanvitelli", Napoli, Italy.

${ }^{7}$ Department of Neuroscience, Rehabilitation, Ophthalmology, Genetics, Maternal and Child Health (DiNOGMI), University of Genova, Italy.

${ }^{8}$ First Division of Neurology, Department of Advanced Medical and Surgical Sciences, Università degli Studi della Campania "Luigi Vanvitelli", Napoli, Italy.

${ }^{9}$ InSpe and Division of Neuroscience, Department of Neurology, IRCCS Ospedale San Raffaele, Milano, Italy.

${ }^{10}$ Neuromuscular Center, Department of Neurosciences Rita Levi Montalcini, University of Torino, Torino, Italy.

${ }^{11}$ Physical Medicine and Rehabilitation Unit, ATS Sardegna, Carbonia, Italy.

${ }^{12}$ Neurology Disease Unit, Osp. S. Chiara APSS, Trento, Italy.

${ }^{13}$ Specialistic Medicine Unit, Azienda Ospedaliero-Universitaria di Parma, Parma, Italy.

${ }^{14}$ IRCCS Istituto delle Scienze Neurologiche di Bologna, Bologna, Italy.

${ }^{15}$ Neurology Unit, Department of Basic Medical Sciences, Neurosciences and Sense Organs, University of Bari, Bari, Italy.

${ }^{16}$ Department of Research and Clinical Development, Scientific Directorate, Fondazione IRCCS Istituto Neurologico Carlo Besta, Milan, Italy.

${ }^{17}$ Department of Medicine and Surgery, University of Parma, Parma, Italy.

${ }^{18}$ Developmental Neurology, Fondazione IRCCS Istituto Neurologico Carlo Besta, Milano, Italy.

${ }^{19}$ Department of Neurological Sciences, Ospedali Riuniti di Ancona, Ancona, Italy.

${ }^{20}$ Marche Polytechnic University, Ancona, Italy.

${ }^{21}$ IRCCS Mondino Foundation, Pavia, Italy.

${ }^{22}$ Department of Neurology/Stroke Unit, San Maurizio Hospital, Bolzano, Italy.

${ }^{23}$ Neurology Unit, Department of Neurosciences, Santa Maria della Misericordia University Hospital, Udine, Italy.

${ }^{24}$ ERN-EURO NMD Center for Neuromuscular Diseases, Unit of Neurology, ASST "Spedali Civili" and University of Brescia, Brescia, Italy.

${ }^{25}$ SS Neurofisiologia, Centro Sclerosi Multipla, Osp. Binaghi, Cagliari, Italy.

${ }^{26}$ Department of Pathophysiology and Transplantation (DEPT), Dino Ferrari Centre, University of Milan, Milan, Italy.

Objective: To retrospectively investigate safety and efficacy of nusinersen in a large cohort of adult Italian SMA patients. Methods: Inclusion criteria were: 1) clinical and molecular diagnosis of SMA2 or SMA3; 2) nusinersen treatment started in adult age ( $>18$ years); 3 ) clinical data available at least at baseline (T0-beginning of treatment) and 6 months (T6). Results: We included 116 patients (13 SMA2 and 103 SMA3) with median age at first administration of 34 years (range 1872). The Hammersmith Functional Rating Scale Expanded (HFMSE) in SMA3 patients increased significantly from baseline to T6 (median change +1 point, $\mathrm{p}<0.0001), \mathrm{T} 10(+2, \mathrm{p}<0.0001)$ and $\mathrm{T} 14(+3$, $\mathrm{p}<0.0001)$. HFMSE changes were independently significant in SMA3 sitter and walker subgroups. The Revised Upper Limb Module (RULM) in SMA3 significantly improved between T0 and T14 (median $+0.5, p=0.012)$, with most of the benefit observed in sitters $(+2, p=0.018)$. Conversely, SMA2 patients had no significant changes of median HFMSE and RULM between T0 and the following time points, although a trend for improvement of RULM was observed in those with some residual baseline function. The rate of patients showing clinically meaningful improvements (as defined during clinical trials) increased from $53 \%$ to $69 \%$ from T6 to T14. Conclusions: Our data provide a further evidence of nusinersen safety and efficacy in adult SMA2 and SMA3, 
with the latter appearing to be cumulative over time. In patients with extremely advanced disease effects on residual motor function are less clear.

Unusual findings detected by diagnostic gene panel sequencing applied to heterogenous neuromuscular disorders

F. Magri ${ }^{1}$, G. Manenti ${ }^{1}$, R. Brusa ${ }^{1}$, P. Ciscato ${ }^{2}$, R. Del Bo ${ }^{1}$, F. Fortunato $^{1}$, S. Lucchiari ${ }^{1}$, S. Pagliarani ${ }^{1}$, D. Piga ${ }^{1}$, D. Velardo ${ }^{2}$, V. Sansone ${ }^{4}$, T. Mongini $^{5}$, S. Gandossini ${ }^{6}$, S. Corti ${ }^{1}$, M. Moggio ${ }^{2}$, G.P.Comi ${ }^{1,2}$, D. Ronchi ${ }^{1}$

${ }^{1}$ Dino Ferrari Centre, Neuroscience Section, Department of Pathophysiology and Transplantation, IRCCS Foundation Ca' Granda Ospedale Maggiore Policlinico, University of Milan, Milan;

${ }^{2}$ Neuromuscular Disorders Unit, Scientific Institute IRCCS E. Medea, Bosisio Parini, Lecco, Italy.

${ }^{3}$ Neuromuscular and Rare Disease Unit, Department of Neuroscience, Foundation IRCCS Ca' Granda Ospedale Maggior e Policlinico, Dino Ferrari Centre, University of Milan, Milan

${ }^{4}$ Centro Clinico Nemo, Niguarda Hospital, Milano, Italy.

${ }^{5}$ Neuromuscular Diseases Unit, Rita Levi Montalcini Department of Neuroscience, University of Torino, Torino, Italy.

${ }^{6}$ Neuromuscular Unit-IRCCS E. Medea Bosisio Parini, Bosisio Parini, Italy.

Diagnosis of neuromuscular disorders is often challenging due to the high variability in clinical presentation and bioptical pattern. Next Generation Sequencing (NGS) approach has been demonstrated to be useful to improve and speed up the achievement of a molecular diagnosis.

We applied a NGS-based gene panel to a cohort of 45 Italian patients presenting neuromuscular disorders. All patients underwent clinical and instrumental evaluation, including muscle biopsy in myopathic cases, neurophysiological studies in the suspect of muscle channelopathy and muscle magnetic resonance imaging, when appropriate.

Our panel include 50 genes covering heterogenous neuromuscular presentations and successfully detected causative mutations in a preliminarily tested validation cohort.

In the main cohort, we detected established or likely pathogenetic mutations in 19 of 45 patients (42\%). Causative variants were found in 13 different genes. Six of them are novel. Specific immunohistochemical and protein studies on muscle specimens were used to investigate the pathogentic role of novel variants.

Unexpected/rare findings include: a missense ANO5 mutation in a case of asympthomatic hyperckemia, a POMGNT2 defect in a mild limb-girdle muscle dystrophic (LGMD) patient, a novel BVES change in a LGMD patient. We also observed a novel missense change affecting the acting biding domain of Filamin-C and biallelic PLEC mutations in two patients with distal myopathy without skin involvement and dystrophic signs. NGS panel might disclose causative molecular defects in classical or peculiar phenotypes when: i) stringent criteria are applied to investigated patients, ii) DNA sequencing is followed by accurate validation studies.

Hind limb unloading as a model of skeletal muscle atrophy: validation of in vivo and ex vivo readouts for preclinical translational research

P. Mantuano ${ }^{1}$, F. Sanarica ${ }^{1}$, O. Cappellari ${ }^{1}$, B. Boccanegra ${ }^{1}$, N. Tarantino $^{1}$, E. Conte ${ }^{1}$, M. De Bellis ${ }^{1}$, G. M. Camerino ${ }^{1}$, S. Pierno ${ }^{1}$, A. De Luca ${ }^{1}$.

${ }^{1}$ Section of Pharmacology, Department of Pharmacy - Drug Sciences, University of Bari "Aldo Moro", Bari, Italy
Skeletal muscle atrophy is a major consequence of several pathophysiological conditions, including neuromuscular diseases, disuse (limb immobilization, space flight), aging (sarcopenia) and other wasting conditions (i.e. cancer cachexia). Basically, a disequilibrium between anabolic/ catabolic pathways leads to progressive loss of muscle mass and functional impairment.

The aim of translational research in this field is to identify anabolic compounds able to support protein synthesis and muscle mass, restoring muscle function and metabolism. Therefore, the validation of readout parameters to assess atrophy/drug efficacy in reliable animal models is pivotal.

Here, we further investigated the hind limb unloading (HU) murine model, where disuse leads to severe atrophy and dysfunction in postural soleus (SOL) muscle [Desaphy et al., Pharmacol Res 2010]. 10-week-old, male C57BL/6J mice underwent a 2-week HU protocol (T0-T2), in comparison to age-, sex-matched non-HU mice for a wide array of in vivo/ex vivo outcomes.

At T0, all mice had comparable body weight (BW). At T2, HU mice BW was significantly lower vs T0 $(-9.7 \%)$ and non-HU mice ($17.3 \%$ ). In vivo torque and ex vivo SOL muscle contraction and elasticity were severely impaired in $\mathrm{HU}$ vs non-HU animals. This was paralleled by a significant decrease in SOL muscle mass $(-39 \%)$ and total protein content $(-47 \%)$. SOL muscle histology was seriously compromised, with clear signs of damage and fiber atrophy. Finally, a notable $-61 \%$ decrease in salivary IgA levels suggested a HU-related negative immune response modulation. Overall, our findings support the usefulness of the HU mouse model for preclinical studies on muscle atrophy.

[Supported by PRIN-MIUR, Prot. 2017FJSM9S]

Long-Term Follow-Up (LTFU) of Onasemnogene Abeparvovec Gene Therapy in Spinal Muscular Atrophy Type 1 (SMA1) From Phase 1 START Trial

J.R. Mendell ${ }^{1-3}$, R. Shell ${ }^{4,5}$, K.J. Lehman ${ }^{1}$, M. McColly ${ }^{1}$, L.P. Lowes ${ }^{1,2}$, L.N. Alfano ${ }^{1}$, N.F. Miller ${ }^{1}$, M.A. Iammarino ${ }^{1}$, K. Church ${ }^{1}$, Raffaele Cerbini $^{6}$, S.P. Reyna ${ }^{6}$, F.G. Ogrinc ${ }^{6}$, H. Ouyang ${ }^{6}$, D.M. Sproule ${ }^{6}$, M. Meriggioli $^{6}$, D.E. Feltner ${ }^{6}$, S. Al-Zaidy ${ }^{6}$

${ }^{I}$ Center for Gene Therapy, Nationwide Children's Hospital, Columbus, $\mathrm{OH}$, United States

${ }^{2}$ Department of Pediatrics, Ohio State University, Columbus, OH, United States

${ }^{3}$ Department of Neurology, Ohio State University, Columbus, OH, United States

${ }^{4}$ Section of Pulmonary Medicine, Nationwide Children's Hospital, Columbus, $\mathrm{OH}$, United States

${ }^{5}$ Department of Pediatrics, Ohio State University, Columbus, OH, United States

${ }^{6}$ AveXis, Inc., Bannockburn, IL, United States

${ }^{7}$ Al-Zaidy and Associates, LLC, Columbus, OH, United States

Introduction: Onasemnogene abeparvovec (formerly AVXS-101) is designed to address the genetic root cause of SMA1. In the phase 1 trial (START; NCT02122952), patients who received a high-dose (proposed therapeutic dose) infusion $(n=12)$ demonstrated significantly improved outcomes versus untreated natural history.

Methods: START patients could electively enroll into LTFU (NCT03421977). Primary objective: long-term safety. Patients have annual visits ( 5 years) followed by annual phone contact (additional 10 years). Assessments include medical history/record review, physical examination, clinical laboratory evaluation, pulmonary assessments, and milestone maintenance.

Results: As of 31 Dec 2019, 13 patients (low dose, $n=3$; therapeutic dose, $\mathrm{n}=10$ ) were enrolled. The oldest patients were aged 6.2 (low dose) and 5.6 
(therapeutic dose) years. Serious adverse events (AEs) were reported in 8/13 patients; no new treatment-related AEs have emerged, supporting a favorable risk:benefit profile. All patients who received the therapeutic dose have survived and are free of permanent ventilation (mean [range] age at last datacut: 4.8 [4.3-5.6] years; mean [range] time since dosing: 4.5 [4.15.2] years). These patients have either maintained all previously attained milestones or gained new milestones; 2 patients have newly achieved standing with assistance while not receiving concomitant SMN2 upregulating therapy at any point. Of the 10 enrolled therapeutic dose patients, 6 require no regular, daily respiratory support more than 4 years after dosing, and 6 are not receiving concomitant SMN2 upregulating therapy.

Conclusions: Data suggest that onasemnogene abeparvovec shows a favorable risk:benefit profile, and continues to demonstrate efficacy with new milestone developments.

\section{Systemic Gene Transfer With rAAVrh74.MHCK7.micro-dystro- phin in Patients With Duchenne Muscular Dystrophy}

JR. Mendell ${ }^{1,2}$, Z. Sahenk ${ }^{1,2}$, K. Lehman ${ }^{1,2}$, C. Nease ${ }^{1,2}$, LP. Lowes ${ }^{1,2}$, NF. Miller ${ }^{1}$, MA. Iammarino ${ }^{1}$, LN. Alfano ${ }^{1}$, J. Vaiea ${ }^{1}$, S. Al-Zaidy ${ }^{1}, S^{\prime}$. Lewis $^{2,3}$, K. Church ${ }^{1}$, R. Shell ${ }^{1}$, L. Picaro ${ }^{3}$, RA. Potter ${ }^{1,3}$, DA. Griffin ${ }^{1,}$ ${ }_{3}$, ER. Pozsgai ${ }^{1,3}$, M. Hogan ${ }^{1}$, LR. Rodino-Klapac ${ }^{1,2,3}$

${ }^{1}$ Center for Gene Therapy, The Abigail Wexner Research Institute at Nationwide Children's Hospital, Columbus, Ohio, USA

2 Department of Pediatrics and Neurology, The Ohio State University, Columbus, OH, USA

${ }^{3}$ Sarepta Therapeutics, Inc., Cambridge, MA, USA

Background: Gene transfer therapy is promising for Duchenne muscular dystrophy (DMD). We designed an adeno-associated virus vector (rAAVrh74) containing a codon-optimized human micro-dystrophin transgene driven by a muscle-cardiac specific promoter, MHCK7. Initial findings from 4 patients in our open-labeled, single-dose, Phase I/IIa trial (NCT03375164) are presented.

Material and Methods: Eligible patients: ambulatory boys (4-7y) with confirmed DMD mutations; creatine kinase (CK) elevations $(>1,000$ $\mathrm{U} / \mathrm{L}) ; \leq 80 \%$ predicted 100 -meter timed test $(100 \mathrm{~m})$; without AAVrh 74 antibodies; stable steroid dosing ( $\geq 3$ months). Patients received an IV infusion $\left(2.0 \times 10^{14} \mathrm{vg} / \mathrm{kg}\right.$ rAAVrh74.MHCK 7.micro-dystrophin). Prednisone $(1 \mathrm{mg} / \mathrm{kg} /$ day $)$ was initiated $1 \mathrm{~d}$ before gene delivery, tapering after 30 days. Primary endpoint: safety. Secondary and exploratory endpoints: micro-dystrophin expression by western blot (WB) and immunohistochemistry (IHC); functional outcomes by North Star Ambulatory Assessment (NSAA), 100m, Time to Rise, 4 Stairs Up; CK levels.

Results: No serious adverse events were observed by serum chemistry. Three patients had transiently elevated gamma-glutamyl transpeptidase (resolved with steroids). No adverse immune responses were observed. Robust transgene expression was observed in all patients, with a mean $81.2 \%$ of muscle fibers expressing micro-dystrophin (mean intensity $96 \%$ at the sarcolemma by IHC). WB mean expression $74.3 \%$ without fat/ fibrosis adjustment (Day 90). Transduction was confirmed in all. Motor function improved in all, measured by increased ambulation $(100 \mathrm{~m})$, increased muscle strength (Time to Rise, 4 Stairs Up), and improvement in overall motor abilities (NSAA). Robust reductions in CK were observed.

Conclusion: Infusion of rAAVrh74.MHCK7.micro-dystrophin was well-tolerated, demonstrating successful systemic delivery of microdystrophin transgene and targeted expression of functional microdystrophin protein product.

Clinico-pathological features in patients with anti-HMGCR immunemediated necrotizing myopathy: a single-center experience
M. Meneri ${ }^{1}$, D. Velardo ${ }^{2}$, M.Magri $^{1,2}$ L. Andreoli ${ }^{1}$, A. De Rosa ${ }^{2}$,R. Brusa ${ }^{1}$, M.Mauri ${ }^{1}$ C. Matinato ${ }^{3}$, L. Peverelli ${ }^{4}$, P. Ciscato ${ }^{2}$, S. Corti ${ }^{1}$, C. Cinnante ${ }^{5}$, M. Moggio ${ }^{2}$, M. Sciacco ${ }^{2}$, GP. Comi ${ }^{1,2}$

${ }^{I}$ Neurology Unit, Neuroscience Section, Department of Pathophysiology and Transplantation (DEPT), Dino Ferrari Centre, University of Milan, IRCCS Foundation Ca' Granda Ospedale Maggiore Policlinico, Milan, Italy.

${ }^{1}$ Neuromuscular and Rare Disease Unit, Department of Neuroscience, IRCCS Foundation Ca' Granda Ospedale Maggiore Policlinico, University of Milan, Milan, Italy.

${ }^{3}$ Laboratory of Clinical Chemistry and Microbiology, Fondazione IRCCS Ca' Granda Ospedale, Maggiore Policlinico, Milan, Italy.

${ }^{4}$ ASST Lodi, Ospedale Maggiore di Lodi distretti e Presidi Lodigiani,U.O. Neurologia.

${ }^{5}$ Neuroradiology Unit, IRCCS Foundation Ca' Granda Ospedale Maggiore Policlinico, Milan, Italy.

Anti-HMGCR immune-mediated necrotizing myopathy is a relatively recently recognized pathological entity, characterized by subacute, progressive, proximal weakness, elevated CK levels and highly specific antiHMGCR autoantibodies. Most patients have a history of exposure to statins, but symptoms fail to resolve after discontinuation of the drug and the majority of patients require immunosuppressive treatment.

In the past three years our clinical chemistry laboratory identified 22 antiHMGCR positive patients. Of these, 13 patients perform periodic followup visits and MRI assessments. Other 3 patients whose initial autoantibody test was made in other laboratories, are regularly re-evaluated in our hospital. Moreover, we had the opportunity to examine 3 other muscle biopsies of anti-HMGCR patients tested positive in our laboratory.

With the exception of a pediatric case, the mean age at onset in the 15 adult patients was 56,87 years (range 34-74, F/M ratio 2/1), with a mean disease duration of 2,08 years (range $0-6$ ). 10 patients received statin therapy. CK mean value at diagnosis was $2503,42 \mathrm{U} / \mathrm{L}$ (range 52-6000), whereas the mean antibody value was 192,2 AU (range 44,8-516,4).

Muscle biopsies showed a pauci-immune necrotizing myopathy with positive MHC-I and, inconsistently, MAC immunohistochemical staining. Whole body muscle MRI highlighted increased STIR signal predominantly involving postero-medial thigh muscles and gluteal compartments, with different degrees of fibro-adipose substitution. Most of the patients needed a long-lasting, at least double, immunosuppressive therapy.

Our cohort expands the knowledge about this rare disease, confirming the existence of a broad phenotypical and biochemical spectrum and the need for early therapeutic intervention.

SUNFISH Part 2: Efficacy and safety of risdiplam (RG7916) in patients with Type 2 or non-ambulant Type 3 spinal muscular atrophy (SMA)

E. Mercuri, ${ }^{1}$ N. Barisic, ${ }^{2}$ O. Boespflug-Tanguy, ${ }^{3}$ N. Deconinck, ${ }^{4,5}$ A. Kostera-Pruszczyk, ${ }^{6}$ R. Masson, ${ }^{7}$ E. Mazzone, ${ }^{1}$ A. Nascimento, ${ }^{8} \mathrm{~K}$. Saito, ${ }^{9}$ D. Vlodavets, ${ }^{10}$ C. Vuillerot, ${ }^{11}$ S. Fuerst-Recktenwald, ${ }^{12}$ S. Fuhrer, ${ }^{13}$ M. Gerber, ${ }^{14}$ K. Gorni, ${ }^{15}$ H. Kletzl, ${ }^{13}$ C. Martin, ${ }^{16}$ W.Y. Yeung, ${ }^{16}$ J.W. Day ${ }^{17}$ on behalf of the SUNFISH Working Group

${ }^{I}$ Paediatric Neurology and Nemo Center, Catholic University and Policlinico Gemelli, Rome, Italy;

${ }^{2}$ Clinical Medical Center Zagreb, University of Zagreb Medical School, Department of Paediatrics, Zagreb, Croatia;

${ }^{3}$ II-Motion - Plateforme d'essais cliniques pédiatriques, Hôpital Armand Trousseau, Paris, France;

${ }^{4}$ Neuromuscular Reference Center, UZ Gent, Ghent; 
${ }^{5}$ Queen Fabiola Children's University Hospital, ULB, Brussels, Belgium;

${ }^{6}$ Department fo Neurology, Medical University of Warsaw, Poland;

${ }^{7}$ Fondazione IRCCS Istituto Neurologico Besta, Developmental Neurology Unit, Milan, Italy;

${ }^{8}$ Neuromuscular UnLit, Neuropaediatrics Department, Hospital Sant Joan de Déu, Fundacion Sant Joan de Deu, CIBERER - ISC III. Barcelona, Spain;

${ }^{9}$ Institute of Medical Genetics, Tokyo Women's Medical University, Tokyo, Japan;

${ }^{10}$ Russian Children Neuromuscular Center, Veltischev Clinical Pediatric Research Institute of Pirogov Russian National Research Medical University, Moscow, Russia;

${ }^{11}$ Department of Pediatric Physical Medicine and Rehabilitation, Hôpital Mère Enfant, CHU-Lyon, Lyon, France;

12 Pharma Development Neurology, F. Hoffmann-La Roche Ltd., Basel, Switzerland;

${ }^{13}$ Roche Pharmaceutical Research and Early Development, Roche Innovation Center Basel, Basel, Switzerland;

${ }^{14}$ Pharma Development, Safety, F. Hoffmann-La Roche Ltd, Basel, Switzerland:

${ }^{15}$ PDMA Neuroscience and Rare Disease, F. Hoffmann-La Roche Ltd., Basel, Switzerland;

${ }^{16}$ Roche Products Ltd., Welwyn Garden City, UK;

${ }^{17}$ Department of Neurology, Stanford University, Palo Alto, CA, USA.

SMA is a severe, progressive neuromuscular disease caused by reduced levels of survival of motor neuron (SMN) protein due to deletions and/or mutations of the SMN1 gene. A second gene, $S M N 2$, produces only low levels of functional SMN protein. Risdiplam is a centrally and peripherally distributed oral SMN2 pre-mRNA splicing modifier that increases levels of functional SMN protein.

SUNFISH (NCT02908685) is a multicenter, two-part, randomized, placebo-controlled, double-blind study (randomized 2:1, risdiplam:placebo) in patients, aged 2-25 years, with Type 2 or Type 3 SMA.

SUNFISH is comprised of two parts: Part $1(n=51)$ is a doseselection study assessing the safety, tolerability and PK/PD of different risdiplam dose levels in patients with Type 2 and Type 3 SMA (ambulant and non-ambulant); confirmatory Part 2 ( $n=180)$ assesses the safety and efficacy of the risdiplam dose level that was selected from Part 1 compared with placebo in patients with Type 2 and non-ambulant Type 3 SMA. The primary objective of Part 2 is to evaluate the efficacy of risdiplam compared with placebo in terms of motor function as assessed by the change from baseline in the 32-item Motor Function Measure total score at Month 12. In SUNFISH Part 1, no drug-related safety findings led to withdrawals from the study following 1 year of treatment with risdiplam (data-cut: $28^{\text {th }}$ June 2019). Here we will report data from Part 2 of the SUNFISH study including baseline demographics, safety and efficacy data in participants who have received treatment with risdiplam or placebo for 12 months.

\section{Improvement of skin biopsy findings after treatment with azatioprina in a case of small fiber neuropathy}

R. Milani ${ }^{1}$, ID. Lopez ${ }^{2}$, A. Quattrini ${ }^{2}$, R. Fazio ${ }^{1}$

${ }^{1}$ Neurology Unit, IRCCS San Raffaele Scientific Institute, Milan, Italy.

${ }^{2}$ Neuropathology Unit, INSPE and Division of Neuroscience, Department of Neurology, Institute of Experimental Neurology, San Raffaele Scientific Institute, Via Olgettina 48, 20132, Milan, Italy

We present an interesting case of a 60 years old man who has been complaining for several years pain, itch and tingling at soles of his feet. He was treated with gabapentin, duloxetina and clonazepam without any benefit. His past medical history revealed a deep vein thrombosis at his right leg and focal epilepsy with many little gliotic foci in white brain matter without enhancement. Laboratory findings showed only a low ANA positivity (1:160). Neurophysiological study was unremarkable. We suspected a small fiber neuropathy possibly related to an inflammatory disorder and a punch skin biopsy at his leg was performed with evidence of severe decrease of the intraepidermal innervation and a Epidermal nerve fiber density (ENFD) of $2.07 / \mathrm{mm}$ (normal value $8.9 \pm$ 5.4). We suspected an inflammatory/ disimmune neuropathy and azatioprina (up to $150 \mathrm{mg}$ per day) was given for six months. Patient reported a pain reduction (VAS 7 to VAS 5). A punch skin biopsy after six months was performed: an almost normal intraepidermal innervation was showed and ENFD was $3.80 / \mathrm{mm}$. These results confirm our suspect of disimmune small fiber neuropathy and suggest that disimmune etiology may be considered in patients with small fiber neuropathy.

Gait and balance evaluation in patients with different myopathies: correlation between imaging data and functional aspects

A.Modenese $^{1}$, A. Picelli ${ }^{1}$, N.Mattiuz ${ }^{1}$, E.Pancheri ${ }^{2}$, N. Smania ${ }^{1}$, G.Vattemi ${ }^{2}$, P.Tonin ${ }^{2}$

${ }^{1}$ Rehabilitation Unit, Verona, Italy

${ }^{2}$ Neurologic Clinic, Verona, Italy

Myopathies have heterogeneous clinical presentations with different muscle involvement and disease progression. The rehabilitation program of these patients usually consists of strength and endurance exercises however, they have an increased fall risk. Posturographic and gait analysis were performed in 32 patients (16 women-16 men, mean age: 49.75 years) affected with different myopathies including myotonic dystrophy, FSHD, LGMD, distal myopathy. Data from clinical-functional tests consisting of Five Times Sit to Stand (5XSST), Timed Up and Go (TUG), Two Minutes Walking Test (TMWT) were collected. Posturography was performed with open and with closed eyes while standing first on a stable surface and then on a compliant support surface. When available MRI cross sectional area (CSA) of lower limb muscles was correlated with gait analysis data.

In all patients we had the following results: 1) increased execution time of the clinical-functional tests, 2) maintenance of the static balance with decreased dynamic equilibrium (double support and stance phase were increased compared to controls), 3) different myopathy-specific gait patterns, 4) a positive correlation between anterior tibial muscle CSA and stance phase, 5) a negative correlation between anterior tibial muscle CSA and the swing phase, 6) a negative correlation between hamstrings CSA and fatigue.

A detailed clinical and instrumental evaluation of patients affected with myopathies is useful to identify specific functional muscle alterations and may help establishing a personalized rehabilitation program including exercises which improve the control of dynamic balance.

Different effects of oxaliplatin and cisplatin incubation on the electrophysiological properties of differentiated F-11 cells

L. Monza ${ }^{1}$, V. Pastori ${ }^{2}$, A. Becchetti ${ }^{2}$, G Cavaletti $^{1}$, M. Lecchi ${ }^{2}$

${ }^{1}$ Experimental Neurology Unit and Milan Center of Neuroscience, School of Medicine and Surgery, University of Milano-Bicocca, Monza, Italy.

${ }^{2}$ Department of Biotechnology and Bioscience and Milan Center of Neuroscience, University of Milano-Bicocca, Milan, Italy.

Chemotherapy-induced peripheral neurotoxicity is one of the most common and often dose limiting side effects of anticancer drugs. Oxaliplatin (OHP) is a third generation platinum compound used as an efficient treatment for 
metastatic colorectal cancer. Unlike other compounds of the same class, OHP may also cause an acute syndrome that seems to be related to the modulation of sodium and potassium channels. Since dorsal root ganglia (DRG) neurons are the main target of platinum-induced peripheral neurotoxicity, we incubated differentiated F-11 cells (rat DRG neurons x mouse neuroblastoma N18TG-2 cell line) for 24 hours in 7,5 $\mu \mathrm{M} \mathrm{OHP}$, and we investigated the electrophysiological properties by patch-clamp technique in the whole-cell configuration. Cisplatin (CDDP $15 \mu \mathrm{M}$ ) was used as reference compound to verify the exclusivity of OHP-induced effects.

In F-11 cells, OHP incubation induced a depolarization of the membrane resting potential $\left(\mathrm{V}_{\text {rest }}\right)$, a reduction of the action potential (AP) firing frequency, an increase of sodium current density and a reduction of ERG (ether-à-go-go-related gene) potassium current density. With regard to ERG potassium and TTX-sensitive sodium currents, OHP shifted both activation and inactivation curves towards more negative potentials and caused an expansion of the window current. In contrast, CDDP treatment induced no effect on $\mathrm{V}_{\text {rest }}$, a decrease of AP firing frequency, an increase of AP duration, and a reduction of sodium, ERG and delayed rectifier potassium current densities.

In conclusion, the collected data indicate that OHP acts on both sodium and potassium voltage-dependent ion channels.

\section{Long-term follow up in presymptomatic LOPD patients (PRELOPD} STUDY). An Italian Neuromuscular Centers Experience.

O. Musumeci ${ }^{1}$, S. Servidei ${ }^{2}$, T. Mongini ${ }^{3}$, S. Ravaglia ${ }^{4}$, G.P. Comi ${ }^{5}$, F. Santorelli ${ }^{6}$,V. Tugnoli ${ }^{7}$, G. Antonini ${ }^{8}$, E. Pennisi ${ }^{9}$, L. Ruggero ${ }^{10}$, G. Siciliano $^{11}$, C. Sancricca ${ }^{2}$, F. Ricci ${ }^{3}$, R. Brusa ${ }^{5}$, A. Rubegni ${ }^{6}$, E. Sette ${ }^{7}$, M. Garibaldi ${ }^{12}$, G. Ricci ${ }^{11}$, A. Toscano ${ }^{1}$

${ }^{1}$ Department of Clinical and Experimental Medicine, Neurology and Neuromuscular disorders Unit, University of Messina - Italy

${ }^{2}$ Fondazione Policlinico Universitario A. Gemelli IRCCS, Istituto di Neurologia Università Cattolica del Sacro Cuore, Rome, Italy

${ }^{3}$ Neuromuscular Unit, Department of Neuroscience "Rita Levi Montalcini", University of Torino, Torino, Italy

${ }^{4}$ Emergency Neurology, IRCCS Mondino Foundation, Pavia, Italy.

${ }^{5}$ Neuromuscular and Rare Diseases Unit, Fondazione IRCCS $\mathrm{Ca}^{\prime}$ Granda Ospedale Maggiore Policlinico, Dino Ferrari Centre University of Milan, Milan, Italy

${ }^{6}$ IRCCS Fondazione Stella Maris, Pisa, Italy

${ }^{7}$ Department of Neuroscience and Rehabilitation, Division of Neurology, University Hospital of Ferrara, via A. Moro, 8, 44100, Cona, Ferrara, Italy

${ }^{8}$ Neuromuscular and Rare Disease Center, Department of Neuroscience, Mental Health and Sensory Organs (NESMOS), SAPIENZA University, Sant'Andrea Hospital, Rome, Italy.

${ }^{9}$ Unit of Neuromuscular Disorders, Neurology, San Filippo Neri Hospital, Rome, Italy.

${ }^{10}$ Department of Neurosciences, Reproductive and Odontostomatological Sciences, University Federico II of Naples, Via Sergio Pansini, 5, 80131 Naples, Italy

${ }^{11}$ Department of Clinical and Experimental Medicine, University of Pisa, Italy

${ }^{12}$ Neuromuscular and Rare Disease Center, Department of Neuroscience, Mental Health and Sensory Organs (NESMOS), SAPIENZA University, Sant'Andrea Hospital, Rome, Italy

Background Late Onset Pompe Disease (LOPD) is characterized by a wide clinical spectrum and may manifest only with hyperCKemia. The increasing disease awareness has allowed earlier LOPD diagnosis at presymptomatic stage. Once identified, it is necessary to establish an adequate follow up to monitor disease progression.

Methods: A survey questionnaire was sent to 20 Italian Neuromuscular Centers asking for LOPD presymptomatic patients followed in their sites. All Centers following at least one presymtomatic patient were invited to fill a database including clinical and functional data collected at T0 (time at diagnosis) and Tlast (time at last follow-up).

Results: We collected data from 26 LOPD patients (17 Males). Mean age at diagnosis was $23,6 \pm 18,2$ yrs, the median follow-up was 7 yrs (range 1-23). $8 / 26$ patients were diagnosed because of positive family history, $18 / 26$ for an isolated hyperCKemia and/or myalgia. First diagnostic approach was: GAA activity on DBS in 4, muscle biopsy in 9, genetic analysis in 10. Muscle biopsy performed in $15 \mathrm{pt}$ revealed a vacuolar myopathy in 9 , was unspecific in 6 pts. Muscle GAA activity ranged from $2 \%$ to $24 \%$. Genetic analysis confirmed diagnosis in all (c.-32-13T>G in $85 \%$ ). Most of the patients were followed up every year with clinical examination, motor functional tests (6MWT and GSGC), pulmonary tests (PFTs) and muscle MRI. 6MWT test at T0 was $540 \pm 51$ and at Tlast $549 \pm 89$. GSGC score at T0 was 4 in 21, still normal at Tlast. PTF showed normal respiratory function (\% predicted sitting FVC at T0: $100 \pm 13$ and at Tlast $96 \pm 14$. Muscle MRI, performed in 17/26 at $\mathrm{T} 0$ was normal in 14 and mildly compromised in 3/17, then remained stable overtime.

Conclusions: Our data demonstrates that the "so-called" presymptomatic LOPD patients could remain clinically silent for ears, although some of them show clinical and laboratory abnormal results. A validation of these clinical and functional assessment could allow clinicians to timely consider when to start therapy in presymptomatic patients.

Whole-exome sequencing identifies recessive RDH11 mutations in a new glycogen storage myopathy with retinitis pigmentosa

O. Musumeci ${ }^{1}$, A. Torella ${ }^{2,3}$, M. Savarese M. ${ }^{4}$, C. Rodolico ${ }^{1}$, A. Ciranni ${ }^{1}$, R. Oteri ${ }^{1}$, F. Del Vecchio Blanco ${ }^{2}$, G. Esposito ${ }^{2}$ G., V. Nigro ${ }^{2,3}$ and A. Toscano ${ }^{1}$

${ }^{I}$ Department of Clinical and Experimental Medicine, Unit of Neurology and Neuromuscular disorders, University of Messina, Messina, Italy

${ }^{2}$ Dipartimento di Biochimica Biofisica e Patologia generale, II Università di Napoli, Napoli, Italy

${ }^{3}$ Telethon Institute of Genetics and Medicine, Pozzuoli (NA), Italy

${ }^{4}$ Folkhälsan Institute of Genetics, University of Helsinki, Helsinki, Finland

Glycogen storage myopathies are heterogeneous disorders characterized by main involvement of skeletal muscle. Nevertheless, they often manifest as multisystemic disorders. In the last decade, significant diagnostic progresses have been achieved with identification of new phenotypes and associated genes because of advances generated by Next Generation Sequencing (NGS) practice

We investigated herein clinical phenotype and genetic origin of a family where two sisters ( 45 and 47 yrs) presenting with myopathy and retinitis pigmentosa (RP) associated to a unique combination of other features, potentially describing a novel syndrome. The clinical history of both patients was remarkably similar. They were born at term but had psychomotor developmental delays since early childhood and complained of fine motor skills and coordination impairment. During infancy, they underwent surgery because of bilateral congenital cataract. Since childhood, they showed a progressive muscle weakness mainly affecting axial and proximal limb muscles. Serum CK was normal in both. Muscle MRI showed a fatty infiltration of thigh muscles mainly in the posterior compartment. Muscle biopsy revealed a vacuolar myopathy with glycogen storage but biochemical analysis failed to show an enzymatic defect of glycolytic or glycogenolytic metabolism.

We performed a step-strategy by NGS: MotorPlex, a NGS custom panel to investigate genes previously associated to skeletal muscle disorders, did not identify any pathogenic mutations. Then, a whole exome approach, performed as second tier test, allowed us to identify the homozygous nonsense R108X mutation in the retinol dehydrogenase 11 gene (RDH11) gene, correctly segregating in the family. Interestingly, a different mutation in this gene, has been already associated in a family with 
RP and multisystemic features with no description of skeletal muscle involvement.

The role of RDH11 is usually related to all-trans-retinoic acid metabolism; its involvement in other organs remains unclear. However, absence of variants in other genes, segregating with the herein described phenotype, suggested that RDH11 mutation could plausibly sustain this new phenotype.

\section{Pure neuritic leprosy: a clinical and neuropathological report}

N. Necchini ${ }^{1}$, S. Cotti Piccinelli ${ }^{1}$, T. Cavallaro ${ }^{2}$, S. Ferrari ${ }^{2}$, B. Risi ${ }^{1}$, E. Baldelli $^{1}$, A. Padovani ${ }^{1}$, M. Filosto ${ }^{1}$

${ }^{1}$ Center for Neuromuscular Diseases, Unit of Neurology, ASST Spedali Civili and University of Brescia, Brescia, Italy

${ }^{2}$ UOC Neurologia B, Department of Neurosciences, AOUI Verona, Verona, Italy

Leprous neuropathy is due to infection of nerve cells by Mycobacterium leprae and it is frequently reported in both lepromatous and tubercoloid type. A pure neuritic form accounts for about $5 \%$ of leprosy cases and can present with single or multiple peripheral nerve involvement. Especially in western countries, pure neuritic leprosy (PNL) is a very rare condition and diagnosis can be misleading.

A 34-year-old Brazilian woman complained of distal sensory impairment at the four limbs for about 2 years. Neurological evaluation showed vibratory, proprioception, tactile and stocking pin-prick sensory impairment in the hands and below the knee. Ankle jerks and knee tendon reflexes were normal as well as general physical examination.

Routine laboratory tests, anti-gangliosides, anti-Borrelia Burgdorferi and anti-Treponema antibodies, immunological tests, cerebrospinal fluid examination and transthyretin gene sequencing yielded normal findings. ENG showed absence of the sural, ulnar and median SAPs. Sural nerve biopsy showed a severe damage characterized by presence of unstructured nerve bundles devoid of nerve fibers, severe inflammatory infiltration and some cells containing acid-resistant Ziehl-Neelsen positive bacilli identified as Mycobacteria leprae.

A treatment based on multidrug therapy was started and patient entered clinical follow-up.

With increasing migration, cases of leprosy are becoming relatively frequent also in western countries. However, PNL still remains a rare and underestimate condition and only nerve biopsy can permit a correct diagnosis.

Since PNL is a treatable disease, it must be suspected in patients from endemic areas presenting with symptoms and signs of peripheral nerve involvement, in order to start the correct therapy as quickly as possible.

\section{NEUROMIO a custom NGS based panel for neuromuscular disor- ders diagnosis: results of the analysis in a cohort of patients}

M.Neri ${ }^{1}$, F.Fortunato ${ }^{1}$, C.Trabanelli ${ }^{1}$, P.Rimessi ${ }^{1}$, R. Selvatici ${ }^{1}$, D.Ognibene ${ }^{1}$,S. Bigoni ${ }^{1}$, S. Fini ${ }^{1}$, E. Terlizzi ${ }^{2}$, D. Giachino ${ }^{3}$, ML Valentino ${ }^{4}$, LM Rocchetti ${ }^{5}$, I .Donati ${ }^{5}$, V.Uliana ${ }^{6}$, E.Pegoraro ${ }^{7}$, A. Pini ${ }^{8}$, M. Pane ${ }^{9}$, F.Gualandi ${ }^{1}$, A. Ferlini ${ }^{1}$

${ }^{1}$ Unit of Medical Genetics, Department of Medical Sciences, University of Ferrara

${ }^{2}$ Department of Neurology, G. Da Saliceto Hospital, Piacenza

${ }^{3}$ Department of Clinical and Biological Sciences, University of Turin, Orbassano

${ }^{4}$ Unit of Neurology, Department of Biomedical and NeuroMotor Sciences (DIBINEM), University of Bologna

${ }^{5}$ Unit of Medical Genetics, Department of Clinical Pathology,

Pievesistina di Cesena

${ }^{6}$ Medical Genetics Unit, University Hospital of Parma
${ }^{7}$ Department of Neurosciences, University of Padua

${ }^{8}$ Child Neurology and Psychiatry Unit, IRCCS Istituto delle Scienze Neurologiche di Bologna

${ }^{9}$ Centro Clinico Nemo, Policlinico A. Gemelli, Fondazione Policlinico Universitario A. Gemelli IRCCS, Rome,

The next-generation sequencing (NGS) technologies has transformed the diagnostic approach of genetic diseases including neuromuscular disorders (NMDs). The molecular genetic diagnosis is indeed compulsory for appropriate clinical management, genetic counselling and family planning and for personalized treatments. As one of the Italian Health Care Provider (HCP) of EURO-NMD we designed a custom panel including 168 genes: 80 of hereditary neuropathies, 46 of congenital myopathies, 39 muscular dystrophies and 3 SLA. We have firstly analyzed 46 patients with different phenotypes : 11 myopathies ( 2 congenital, 2 adult, 1 distal, 3 with contractures, 1 with myofibrilles, 1 with muscle hypertrophy and 1 with cardiomyopathy), 11 muscular dystrophies, 3 neonatal hypotonia, 14 peripheral neuropathies, 3 high CK , 2 collagenopathies and 2 motoneuron disease. The analysis identified in six patients pathogenic gene variations: a de novo ACTA1 mutation in patient with myopathy and contractures, an homozygous CAPN3 mutation in a LGMD patient, biallelic ANO5 mutations in a CK phenotype, biallelic DYSF mutations in a muscular dystrophy patient, a DYNC1H1 mutation in a SMALED phenotype and a COL6A1 mutation in a collagenopathy. In 4 patient we have identified likely pathogenic variations and is ongoing the segregation analysis in the family. In 15 patients the analysis identified variants of uncertain significance (VUS) and in 21 patients was fully negative. We analyzed a small group of patients therefore it is not possible to conclude about NEUROMIO diagnostic sensitivity. We suggest that our panel can be useful to pre-screen a number of genes for which a rapid diagnosis might be necessary (when an orphan drug is available or clinical trials are running). In other cases, the future genetic tool with the higher sensitivity will certainly be whole genome sequencing (WGS) which is in course of diagnostic validation.

\section{Characteristics of corneal innervation in patients with Myasthenia Gravis}

G. Nicocia ${ }^{1}$, C. Bonanno ${ }^{1}$, A. Pugliese ${ }^{1}$, D. Montanini ${ }^{2}$, E. Postorino ${ }^{2}, \mathrm{~S}$.

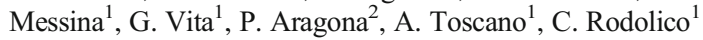

${ }^{1}$ Department of Clinical and Experimental Medicine, Unit of Neurology and Neuromuscular diseases, University of Messina, Messina, Italy ${ }^{2}$ Department of Adult and Development Age Human Pathology "Gaetano Barresi", Unit of Ophthalmology, University of Messina, Messina, Italy

Background: Many MG patients report blurring of vision and sensation of "dry eye", in spite of a good control of neuromuscular symptoms. Few studies reported that ocular surface in MG is quite similar to dry eye in connective tissue disorders.

Aim: The aim of this study is to evaluate the characteristics of sub-basal cholinergic nerves in a cohort of MG patients.

Patients and methods: $27 \mathrm{MG}$ patients $(13 \mathrm{M}, 14 \mathrm{~F})$ and 20 controls with dry eye $(10 \mathrm{M}, 10 \mathrm{~F})$ were enrolled. All underwent Cochet-Bonnet aesthesiometry and in vivo confocal microscopy.

Results: mean age of MG patients was 57,6 $\pm 16,5$ years and mean age of controls was $36 \pm 14,8(\mathrm{P}=0,2)$. According to MGFA classification, 14 had MG-IIA,7 IIB, 1 IIIA, 3 IIIB, 2 type I. AChR antibodies were elevated in 24. The mean corneal touch threshold (CTT) in MG group $(13,22 \mathrm{mg} \pm 8,3)$ differed significantly from dry eye group $(11,2 \mathrm{mg} \pm 0,4)$ with a $\mathrm{p}$ value of 0,02 .Total number of nerves in the corneas of controls were significantly lower than in MG $(2,5 \pm 1,2$ vs 4,5 $\pm 2,9$ respectively; $p=0,008)$. Nerve beadings and density in $\mathrm{MG}(57,7 \pm 27,9$ and $80,6 \# / \mathrm{mm} \pm 9,4$ respectively) 
were significantly higher than in controls $(40,3 \pm 18$, and $71,9 \# / \mathrm{mm} \pm 14,2$ respectively) with a $\mathrm{p}$ value of 0,02 and of 0,01 respectively.

Conclusions: We speculate that blurring of vision and sensation of "dry eye" in MG underlie a different pathogenesis. An increased number of beadings in the corneas of MG group could be related to a compensatory mechanism due to a chronic ocular motor impairment.

Long-term impact of inotersen on neuropathy quality of life (QoL) for hereditary transthyretin amyloidosis with polyneuropathy (hATTR-PN): NEURO-TTR open-label extension at 2 years

L. Obici ${ }^{1}$, Andrew Lovely ${ }^{2}$, Teresa Coelho ${ }^{3}$, Aaron Yarlas ${ }^{2}$, Michael Pollock $^{4}$, Kristen McCausland ${ }^{2}$, Isabel Conceição ${ }^{5}$, Chafic Karam ${ }^{6}$, Sami Khella ${ }^{7}$, G.Vita ${ }^{8}$, M. Waddington-Cruz ${ }^{9}$, M. V. Llonch ${ }^{4}$

${ }^{1}$ Amyloidosis Research and Treatment Center, Fondazione IRCCS Policlinico San Matteo, University of Pavia

${ }^{2}$ Optum

${ }^{3}$ Hospital Santo António, Centro Hospitalar do Porto

${ }^{4}$ Akcea Therapeutics

${ }^{5}$ Hospital de Santa Maria, CHULN, Faculdade de Medicina,

${ }^{6}$ Department of Neurology, Oregon Health \& Science University,

${ }^{7}$ University of Pennsylvania,

${ }^{8}$ Azienda Ospedaliera Universitaria Policlinico "G. Martino", Messina,

${ }^{9}$ Hospital Universitário Clementino Fraga Filho, Universidade Federal do Rio de Janeiro

Background: In the randomized, controlled phase 3 NEURO-TTR trial, patients with hATTR-PN receiving inotersen experienced statistically significant improvements on neuropathic-specific QOL versus placebo. After trial end, all consenting patients were switched to inotersen onceweekly in an open-label extension (OLE).

Objective: Examine changes in Norfolk QOL Diabetic Neuropathy (QOL-DN) survey domain scores after 2 years of OLE treatment.

Methods: The Norfolk QOL-DN captures neuropathic-specific activities of daily living (ADL), autonomic neuropathy, large fiber neuropathy (LFN), small fiber neuropathy (SFN), and symptoms. It was administered at OLE baseline and weeks 26, 78, 104. Descriptive analyses examined mean domain scores.

Results: The OLE enrolled 135/139 patients originally receiving inotersen (inotersen-inotersen; $n=85$ ) or placebo (placebo-inotersen; $n=50$ ). For both subgroups, Norfolk QOL-DN domain scores from OLE baseline to week 104 were stable: ADL (mean changes $=2.0,2.3$ points, respectively), autonomic neuropathy $(0.3,0.1), \operatorname{LFN}(2.1,1.5), \operatorname{SFN}(0.2,1.2)$, and symptoms domains $(0.4,-0.5)$. Subgroup differences at OLE baseline persisted by week 104: the mean difference for the inotersen-inotersen and placebo-inotersen subgroups was 2.2 at OLE baseline vs. 2.4 at week 104 for ADL, 0.7 vs. 0.5 for autonomic neuropathy, 7.2 vs. 6.6 for LFN, -0.3 vs. 0.8 for SFN, and 2.5 vs. 1.6 for symptoms.

Conclusions: Treatment with inotersen over 2 years stabilized neuropathic-specific QOL for patients with hATTR-PN. The gaps in QOL between those receiving inotersen versus placebo during NEURO-TTR were sustained even 2 years after inotersen initiation in the placebo subgroup, underlining the importance of early treatment.

CPPs-conjugated antisense nucleotides: a new therapeutic strategy for Spinal Muscular Atrophy symptomatic patients

E. Pagliari ${ }^{1}$, M. Bersani ${ }^{1}$, M. Rizzuti ${ }^{2}$, A. Bordoni ${ }^{2}$, D. Saccomanno ${ }^{2}$, N. Bresolin $^{1,2}$, GP. Comi ${ }^{1,2}$, S. Corti ${ }^{1,2}$, M. Nizzardo ${ }^{1}$

${ }^{1}$ Università degli Studi di Milano, Department of Pathophysiology and Transplantation (DEPT), Milano, Italy
2 IRCCS Ca' Granda Ospedale Maggiore Policlinico, Neurology Unit, Milano, Italy

Spinal muscular atrophy (SMA) is a motor neuron disease caused by mutations in the Survival Motor Neuron 1 (SMN1) gene, resulting in deficiency of SMN protein. Most of the emerging therapies are based on redirecting the splicing of $S M N 2$, the paralogous gene, to produce a functional SMN protein. In our laboratory, a specific sequence of the antisense oligonucleotide Morpholino (MO) against the ISS-N1 region of SMN2, the MO-10-34, has been successfully tested in presymptomatic SMA mice. To develop a functional treatment for symptomatic patients, cell-penetrating peptides (CPPs) can be conjugated to ASOs, allowing the crossing of the blood-brain barrier (BBB). This approach has been preliminarily explored with MO conjugated with four different peptides in pre-symptomatic mice, demonstrating the major efficacy of $\mathrm{r} 6$ and RXR, on which we focused further experiments. To verify the ability of MO-conjugated to CPPs to cross the BBB and ameliorate SMA mice phenotype and neuropathological features compared to unconjugated$\mathrm{MO}$, two group of symptomatic mice animals were treated at $\mathrm{p} 5$ with r6-MO, RXR-MO and unconjugated MO by intraperitoneal injection. The first group was monitored for survival and phenotypical behaviour. The second group was sacrificed at p30, spinal cord and intercostal muscles were harvested and analysed by immunofluorescence for the presence of motor neurons and innervated neuro muscular junctions. Our results confirm that both CPPs-conjugated MOs ameliorate the biodistribution of the MO into the CNS increasing significantly phenotypical and neuropathological features compared to unconjugated MO.

Familial Progressive Cardiac Conduction Disease caused by a TRPM4 gene mutation.

A. Palladino ${ }^{1}$, Torella ${ }^{2}$, R. Petillo ${ }^{1}$, A. M. Scutifero ${ }^{1}$, S. Morra ${ }^{1}$, P. D’Ambrosio $^{1}$, L. Passamano ${ }^{1}$, V. Nigro ${ }^{1,2}$ and L. Politano ${ }^{3,4}$

${ }^{1}$ U.O.i.D. di Genetica Medica e Cardiomiologia, AOU dell'Università della Campania Luigi Vanvitelli - Napoli, Italy;

${ }^{2}$ Dipartimento di Medicina di Precisione, Università della Campania Luigi Vanvitelli - Napoli, Italy;

${ }^{3}$ Centro Gaetano Torre per le Malattie Muscolari, Unità di Ricerca Napoli, Italy;

${ }^{4}$ Centro Clinico NeMO “Giovanni Nigro” - Napoli, Italy

Progressive cardiac conduction disease (PCCD) is a relatively common condition in young and elderly populations, related to rare mutations in several genes including SCN5A, SCN1B, TRPM4, LMNA and GJA5. Familial cases have also been reported, suggesting predisposing factors.

We describe a three-generation family with a high number of individuals presenting several cardiac conduction defects, as Atrio-Ventricular (AV) or right bundle branch block (RBBB).

The proband is a 46 year-old-patient, whose younger brother has died at the age of 25 for sudden cardiac death during sleep. In the paternal lineage, to report 3 male uncles implanted with a pacemaker at an age between 40 and 65 years. The patient has not completed the military service for the detection of a RBBB. At the age of 42, he was implanted with a pacemaker for the presence of a complete atrio-ventricular block. The NGS genetic analysis revealed a heterozygous missense mutation (p.Gly844Asp) in the TRPM4 gene, that segregates with the disease. This gene encodes the TRPM4 channel, a calcium-activated nonselective cation channel of the transient receptor potential melastatin (TRPM) ion channel family.

The same mutation has been reported in 2010 by Liu et al. in 9 connected individuals presenting with different degrees of right bundle branch block. The mutation results in an increased current density at the cell surface secondary to impaired endocytosis and deregulation of Small Ubiquitin MOdifier conjugation (SUMOylation), causing a gain of function of the gene. Our report broadens and supports the causative role of TRPM4 mutations in 
PCCD.

A de novo in-frame deletion in MYOT causes an early adult onset severe distal myopathy

E. Pancheri ${ }^{1}$, V. Guglielmi ${ }^{1}$, V. Nigro $^{2}$, S. Aurino ${ }^{2}$, A. Torella ${ }^{2}, \mathrm{M}$. Malatesta $^{3}$, A. Vettore ${ }^{4}$, A. Giorgetti ${ }^{4}$, G. Tomelleri ${ }^{1}$, P. Tonin ${ }^{1}$, G. Vattemi $^{1}$

${ }^{1}$ Department of Neurosciences, Biomedicine and Movement Sciences, Section of Clinical Neurology, University of Verona, Verona, Italy

${ }^{2}$ TIGEM (Telethon Institute of Genetics and Medicine), University at Campania, Naples, Italy

${ }^{2}$ Department of Neurosciences, Biomedicine and Movement Sciences, Section of Anatomy and Histology, University of Verona, Verona, Italy

${ }^{4}$ Department of Biotechnology, University of Verona, Verona, Italy

The myotilin gene (MYOT) encodes a $57-\mathrm{kDa}$ sarcomeric Z-disk protein which is expressed in skeletal and cardiac muscle and in peripheral nerves. Mutations in MYOT have been originally associated with different disorders separated, according to the clinical and histological findings, in three main categories including autosomal dominant limb girdle muscular dystrophy type 1A (LGMD 1A), myofibrillar myopathy (MFM) and spheroid body myopathy. We describe an Italian male patient and his mother presenting with a severe distal myopathy caused by the first deletion c. 11_28del in the MYOT gene in a heterozygous state. The proband's muscle biopsy showed histological features suggestive for a diagnosis of myofibrillar myopathy and a biochemical study documented expression levels of myotilin in the skeletal muscle of the patient similar to those in control muscles. The pathogenicity of the described deletion was further confirmed using an in vivo modeling in zebrafish.

\section{Issues in the management of hereditary transthyretin-mediated am-} yloidosis (hATTR)

A. Parachini ${ }^{1}$, B. Valzasina ${ }^{2}$, M. Grandis ${ }^{3}$, M. Oliverio ${ }^{1}$, A. Schenone ${ }^{3}$, L. Obici ${ }^{4}$

${ }^{1}$ Doxa Pharma Srl;

${ }^{2}$ Akcea Therapeutics Italy Srl;

${ }^{3}$ Unità Operativa Clinica Neurologica, IRCCS Ospedale Policlinico San Martino, Genova;

${ }^{4}$ Amyloidosis Center, IRCCS Policlinico San Matteo, University of Pavia, Italy

hATTR is a rare disease characterized by the deposition of amyloid fibrils in various organs and tissues, leading to disability and mortality.

Aims: To highlight patients' needs, diagnostic processes and impact on QoL, as perceived by patients and clinicians in real-life.

Methods: Online questionnaire for participants (15 clinicians and 6 hATTR patients/caregivers) and innovative qualitative interviews for 6 patients/caregivers. Prior to in-depth interviews, participants completed a scrapbook to spontaneously describe the highly emotional, personal dimensions of the disease.

Results: The survey reveals differences between patients and clinicians' perception of the impact of the disease which concerns different aspects. The first aspect regards the clinician-patient relationship: while patients report not to be fully satisfied regarding communication with their clinicians, the latter hold the view they share exhaustive information about the condition and its progress/treatment. Secondly, regarding genetic testing, although physicians propose it for every at-risk relative and ask for strict follow-up, patients are often reluctant to accept or limit it to few relatives.
Finally, the disease has a high psychological burden, follow-up heavily impacts on QoL, patients desire to be managed by a multidisciplinary team. They ask for GPs/local specialists training for early symptoms recognition to shorten time to diagnosis, information campaigns on genetic tests, physiotherapy to improve mobility and/or pain and psychological support.

Conclusion: hATTR heavily impacts patients' life, beyond physical impairments; an easier way to diagnosis, the possibility to rely on a multidisciplinary team, better information and psychological support are current unmet needs. A limitation of the current study is that there is no oneto-one relationship between patients/carriers and physicians interviewed.

Clinical and electrophysiological features of seipinopathy in an Italian family: an example of spontaneous regressive course of the disease - a case report

\author{
S. Parravicini ${ }^{1,2}$, A.Asaro ${ }^{1,2}$, C.Cereda $^{1,2}{ }^{\text {, }}$ A.Lozza $^{1,2}$, A. Berardinelli ${ }^{1}$ \\ ${ }^{1}$ stituto Neurologico IRCCS C. Mondino, Pavia, Italy \\ ${ }^{2}$ Università degli Studi di Pavia
}

Introduction: mutations of the BSCL2 gene were identified as the cause of a group of heterogeneous diseases that were proposed to be classified as seipinopathies. This group includes a congenital generalized lipodystrophy (Berardinelli-Seip Syndrome) and motor neuron seipinopathies with an autosomal dominant transmission, caused by heterozygous gain-of-function mutations. The latter group can be furtherly subdivided into different phenotypes: dHMN-V, Silver syndrome/ SPG17, and CMT2. Besides the conditions described above, the clinical spectrum also includes asymptomatic mutation carriers, subclinically affected patients, ALS-like rapid progressive and MMN-like phenotypes. This phenotypic variability has been reported both between and within families.

Case presentation: here we describe two patients from an Italian family with a BSCL2 mutation (p.N88S): a boy, presenting a dHMN-V clinical picture and his affected father, who was asymptomatic at the time of genetic diagnosis and unexpectedly reported a clinical history of a spontaneously remitting course of the symptoms.

Discussion: the broad clinical spectrum of the manifestations of motor neuron seipinopathies has been described, as well as the clinical heterogeneity between and within families. Up to now, the cases described in the literature showed a progressive or stable course of the disease. Interestingly, the patient 2 of our report spontaneously switched from subclinical to asymptomatic state. To the best of our knowledge, it is the first described case of a spontaneously regressive course of motor neuron seipinopathies. The evidence of a possible regressive course of the disease may add further variability to the broad clinical spectrum of seipinopathies, additionally supporting the role of possible genetic modifiers.

\section{Response to treatment and outcome in late versus early onset MG}

F. Pasqualin ${ }^{1}$, S.V. Guidoni ${ }^{1}$, M. Ermani ${ }^{2}$, E. Pegoraro ${ }^{2}$, D.M. Bonifati ${ }^{1}$

${ }^{1}$ Unit of Neurology of Ca' Foncello Hospital, 31100 Treviso (Italy)

${ }^{2}$ Department of Neuroscience, University of Padova, 35128 Padova, Italy.

Background and objectives: Recently different subtypes of myasthenia gravis (MG) have been described. They differ for clinical features and pathogenesis but the prognosis and response to treatment is less clear. The aim of the study was to evaluate treatment, outcome and side effects in early versus late MG. 
Methods: We analysed retrospectively 208 MG patients. Clinical features were recorded as well as treatment, side effects and outcome at the last follow-up using MGSTI and MGPIS scales.

Results: the 208 patients included in the study were classified as follow: 36 ocular MG, 40 early onset MG (EOMG), 72 late onset (LOMG), 25 thymoma, 14 anti-MuSK and 21 double seronegative. Similar positive outcome was achieved in the two groups: pharmacological remission and minimal manifestations at the MGFA-PIS in the 95\% and 94,4\% in EOMG and LOMG respectively but in LOMG lower dose of immunosuppressors $(\mathrm{MGSTI}<2)$ was required compared to EOMG $(\mathrm{p}=0,048)$. Severe side effects were present in a small percentage of patients in both group but diabetes was more frequent in LOMG vs EOMG $(2,2 \%$ vs $5 \%, \mathrm{p}=0.017)$.

Conclusions: Despite LOMG had more comorbidities that might interfere with treatment, therapeutic management did not differ between EOMG and LOMG, with similar positive outcomes. The LOMG group required lower doses of medication to control symptoms. The prevalence of side effects did not differ between EOMG and LOMG patients but in EOMG side effects such as cataract, diabetes and osteoporosis were more frequent compared to the general population.

Keywords: MGFA-PIS, MGSTI, myasthenia gravis, characterization, outcome

\section{Next generation sequencing-based gene panel tests for the manage-} ment of diagnosis of lipid myopathies.

E.M. Pennisi, F. Cortese, F. Gragnani, L. DeGiglio, M. Garibaldi, A. D'amico, E. Bertini, F. Fattori.

\author{
${ }^{1}$ Neuromuscular Center, Neurology Unit, San Filippo Neri Hospital, \\ Rome. \\ ${ }^{2}$ Neurology Unit, Pertini Hospital, Rome \\ ${ }^{3}$ Neuromuscular Center, Neurology Unit, S. Andrea Hospital, La \\ Sapienza University, Rome \\ ${ }^{4}$ Neuromuscular and Neurodegenerative Diseases Unit - Molecular \\ Medicine Laboratory, IRCCS Bambin Gesù Pediatric Hospital, Rome.
}

Lipid myopathies (LM) are a group of muscular diseases with onset in all ages, owing in most cases to enzymatic errors of lipid metabolism. LM can be, but not always, characterized by lipid storage in muscle biopsy. Clinically LM can show stable and progressive weakness or episodic asthenia with acute rhabdomyolysis, triggered by metabolic stress, fever, physical exercise. The diagnostic approach includes clinical evaluations, biochemical tests (serum acylcarnitine esteres), and genetic analysis of the suspected gene. Although lipid myopathies are potentially life-threatening diseases, some types of lipid myopathy can be treated with adequate therapy. Nevertheless, diagnosis is often complicated for the similarity of clinical features with other myopathies that may show a pseudometabolic syndrome like GMPPB, RYR1, etc and thus interfering with figures of prevalence. To overcome this diagnostic hurdle, we designed a next-generation sequencing (NGS) panel that assesses genes associated with lipid disorders in muscle tissue adding these to our custom panel for myopathies that includes 110 genes. The criteria for including candidate patients for this test are adults or infants who manifest one or more of these features: rabdomyolisis, excercise intolerance, myalgia, transient myopathy or fixed myopathy with lipid storage or ichthyosis, ichthyosis, and some other disoders such as cardiomyopathy, metabolic encephalopathy, hepatopathy, hypoglycemia, metabolic acidosis neonatal hypotonia, Reye like syndrome. In case of negative results we will proceed to WES. The panel for myopathies with include 17 genes to date associated to LM: SLC22A5, PNPLA2, CGI58/ABHD5, LPIN, SLC25A20, CPTII, ACADVL, ACAD9, ACADL, ACADM, ACADS, ADHA, ADHB, HADH, MCKAT, ETFA, ETFB, ETFDH.

Lipid composition of cellular membranes in Neutral lipid Storage Disease type M: a possible role in etiology of disease?
E.M. Pennisi ${ }^{1}$, D. Tavian $^{2}$, N.I. Noguera ${ }^{3}$, L. DeGiglio ${ }^{1}$, L., M. Mora ${ }^{4}$, L.Maggi ${ }^{4}$, M. Filosto ${ }^{5}$, Cortese $\mathrm{F}^{1}$, M.Garibaldi ${ }^{6}$, S.Missaglia ${ }^{2}$, C.Angelini ${ }^{6}$, E.Palma ${ }^{7}$, A.Macone ${ }^{7}$.

${ }^{1}$ San Filippo Neri Hospital, Rome

${ }^{2}$ CRIBENS, Catholic University, Milan

${ }^{3}$ Santa Lucia Foundation IRCCS, Rome

${ }^{4}$ I. Besta IRCCS, Milan

${ }^{5}$ ASST Spedali Civili, University of Brescia, Brescia

${ }^{6}$ San Camillo Foundation IRCCS, Venice

${ }^{7}$ La Sapienza University, Rome

Patients with mutations in the PNPLA2 gene, that codifies for ATGL, suffer from a rare defect in triglycerides catabolism that reduces their release in cytoplasm and causes the neutral lipid storage disease type M (NLSD-M) characterized by severe myopathy and cardiomyopathy. Amost 100 patients have been diagnosed worldwide. The pathogenesis of muscle damage in NLSD-M is not still well defined. Some studies report a possible metabolic damage, but structural defects of cellular organelles could have a role in muscular atrophy. Lipids represent the major building blocks for the synthesis of neo-generated membranes, besides having their well-known role as energy storage. Triglycerides are crucial precursors for the synthesis of membrane lipids. We hypothesize that the cytoplasmatic regulation of lipid catabolism could play a pivotal role in the phospholipid composition and in building cellular membranes. We studied 5 patients with NLSD-M and 3 controls. Membrane phospholipids have been extracted and fatty acid methyl esters were measured by GC. The preparation for analysis contained cytoplasmatic membranes and a small amount of reticulum endoplasmic. Nuclear membranes have been excluded. The membranes of the patients showed a significant lacking of medium chain FA, in particular $\mathrm{C} 12$ and $\mathrm{C} 14$ respect to normal membranes, while fatty acids with longer chains did not show significant differences. Additional experiments with patch-clamp recording showed no evidence of alteration of channels in fibroblast membranes. However, fibroblasts, being not contractile cells, may not express significant alterations in channels. Our results indicate a possible structural alteration of membranes composition in the ATGL deficiency.

Isolated cranial neuropathy associated with anti-glycolipid antibodies

\section{Petrelli, F. Logullo}

\section{UOC Neurologia, Ospedale di Macerata ASUR Marche, Italy}

Cranial nerve mononeuropathies are frequently found in clinical practice and their cause is often unknown. We describe 4 patients with isolated cranial neuropathy in whom serum anti-glycolipid antibodies were detected. 2 patients presented with hypoglossal neuropathy and 2 patients presented diplopia; the orthoptic evaluation revealed deficiency in one case of the right rectus lower eye muscle, in the other case of the upper rectus muscle of right eye. The onset of symptoms was acute in all 4 patients, preceded about 2 weeks earlier by infection. They underwent appropriate examinations: Magnetic Resonance Imaging (MRI) studies, laboratory tests to exclude metabolic disease and other neurological disease, various myopathies and myasthenia gravis, electrophysiological studies to examine the presence of a sensory-motor polyneuropathy. Sera samples were screened: oculomotor nerve neuropathy: case 1 GD1a ( $\mathrm{IGg}$ ), case 2 GM1 (IgM); hypoglossal nerve neuropathy: case 3 GD $1 \mathrm{~b}(\mathrm{IgG})$, case $4 \mathrm{GM} 1(\mathrm{IgM})$ and anti-sulfatide antibodies (IgG). The hypoglossal neuropathy was confirmed by 
electrophysiological study. Several antibodies against glycolipid play important roles in the development of peripheral nervous damage and clinical spectrum of Guillain-Barré Syndrome (GBS) is suggested to be associated with the reactivity of anti-glycolipid antibodies. Polyneuritis cranialis is considered as a variant of GBS. However, little is known about the clinical significance of antiglycolipid antibodies in other isolated cranial neuropathies. We speculate that the present cases share similar immunopathogenesis to GBS, some anti-glycolipids antibodies are presented as pathogen, however, different immune responses from those in GBS are based on the development of isolated cranial neuropathy.

Clinical and Genetic Characteristics of NEFL-related CharcotMarie tooth disease due to $\mathrm{P} 440 \mathrm{~L}$ mutation in a large Italian family

A. Petrucci ${ }^{1}$, L. Lispi ${ }^{1}$, M. Garibaldi ${ }^{2}$, E. Frezza ${ }^{3}$, R. Massa ${ }^{3}$, F. Moro ${ }^{4}$, FM. Santorelli ${ }^{4}$

${ }^{1}$ Center for Neuromuscular and Neurological Rare Diseases, San Camillo Forlanini Hospital, Rome, Italy

${ }^{2}$ Neuromuscular and Rare Diseases Center, Department of Neuroscience, Mental Health and Sensory Organs (NESMOS), Sapienza University, Sant'Andrea Hospital, Rome, Italy

${ }^{3}$ Neuromuscular Diseases Unit, Department of Systems Medicine, Tor Vergata University of Rome, Rome, Italy.

${ }^{4}$ Molecular Medicine for Neurodegenerative and Neuromuscular Diseases Unit, IRCCS Stella Maris Foundation, Pisa, Italy.

Gene mutations in neurofilament light (NEFL) account for $<1 \%$ of all Charcot-Marie-Tooth (CMT) cases, with different phenotypes, including demyelinating (CMT1F) and axonal (CMT2E) neuropathies; dominant and recessive inheritance have been described (1). Here we describe an Italian family, affected by sensory-motor axonal polyneuropathy, with dominant inheritance; genetic analysis revealed two heterozygous variants in NEFL gene: - E488K and P440L. Only missense P440L variant segregated with the phenotype in seven individuals, 23- 62 years old; symptoms onset was in childhood in two, with running difficulties, and in adulthood in 5 patients, for walking complains. Clinical features were: absent/reduced deep tendon reflexes in all patients, foot deformities in three, impaired gait on heels in six, reduced sensation in lower limbs in five, distal weakness in upper and lower limbs in one and in lower limbs in four, sensorineural hearing loss in four patients; other features were represented by cardiac conduction abnormalities at two years of age in one patient, requiring pacemaker implantation two years later; brain MRI resulted in normal findings in all patients and spinal MRI documented dorsal syringomyelia in one patient. A previous report documented P440L NEFL mutation in an autosomal dominant family with axonal CMT; however the segregation was unclear, pyramidal tract signs were reported and some patients showed no sensory involvement (2); in our family the segregation is well documented, no pyramidal signs were detected and hearing loss was a common finding such as in other NEFL mutations; the cardiac abnormalitie showed by one patients is unusual finding for CMT. This report confirms P440L mutation in the pathogenesis of NEFL related CMT and expands its phenotype.

(1) Genetic and clinical characteristics of NEFL-related Charcot-MarieTooth disease. A. Horga et al. J Neurol Neurosurg Psychiatry 2017;0:111.

(2) Imrpoved inherited peripheral neuropathy genetic by whole-exome sequencing. AP Drew et al. Molecular Genetics and Genomic Medicine 2015;3(2):143-154.

Unusual case of motor and dysautonomic neuropathy due to thallium intoxication
A. Petruzzellis ${ }^{1}$, E. Vecchio ${ }^{1}$, L. Gallicchio ${ }^{1}$, V. Recchia ${ }^{1}$, L. Didonna ${ }^{1}$, P. Lovreglio ${ }^{2}$, G. De Palma ${ }^{3}$, F. Tamma ${ }^{1}$

${ }^{1}$ UOC Neurologia, Ente Ecclesiastico Ospedale F. Miulli, Acquaviva delle Fonti (Ba), Italy

${ }^{2}$ Dipartimento Interdisciplinare di Medicina-Sezione di Medicina di Lavoro "EC Vigliani" Università di Bari Aldo Moro, Italy

${ }^{3}$ Dipartimento di Specialità Medico Chirurgiche Scienze Radiologiche e Sanità Pubblica, Sezione di Sanità Pubblica e Scienze Umane, Università di Brescia, Italy

Thallium poisoning is one of the most complex and serious toxicities. The symptomatology is nonspecific due to the multi-organ involvement and difficult to diagnose in the absence of a clear exposure.

In October 2018, a 24 years patient was admitted in hospital presenting four limbs paresthesia, alopecia, thoracic neuropathic pain, hypertension and tachycardia followed by constipation. ENG- EMG and neurological examination were normal. Hematological tests ruled out porphyria and symptoms recovered spontaneously. After 3 months, he had a recurrence. ENG- EMG was still normal and DNA test for porphyria was negative. He started olanzapine and paroxetine with no benefit. In May 2019, he was admitted to our hospital with rapidly progressive flaccid paraplegia.

ENG-EMG was consistent with acute motor axonal neuropathy in lower limbs, while CSF showed mild increased protein level. We administered IvIg $(2 \mathrm{gr} / \mathrm{kg})$ with no benefit, while we observed progressive electrophysiological and clinical involvement of upper limbs. We hypothesized a toxic cause and we dosed blood and urine heavy metals.

Thallium blood level was markedly increased (129 $\mu \mathrm{g} / \mathrm{l})$. Therapy with Activated Carbon and Prussian Blue was given orally and was discontinued when thallium blood and urine levels became undetectable.

Electrophysiological and clinical follow up showed progressive improvement in CMAP amplitude and limbs strength. At the last visit, he was walking with ankle foot orthosis, while alopecia and autonomic dysfunction were fully recovered. The poisoning source is still unknown.

Rare toxic causes can always be responsible for cases of a motor-sensitive neuropathy with signs of autonomic dysfunction.

Acquired asymmetric brachial plexopathy: a description of a caseseries

E. Pezzotti, L. Pasca, A. Gardani, V. Vacchini, E. Rognone, M. Paoletti, G. Cosentino,M. Plumari, C. Cereda, A. Berardinelli.

- Child Neurology and Psychiatry Unit, IRCCS Mondino Foundation and University of Pavia, Pavia, Italy.

- Department of Neuroradiology, IRCCS Mondino Foundation, Pavia; Institute of Radiology, University of Pavia.

- Clinical Neurophysiology Unit, IRCCS Mondino Foundation, Pavia, Italy.

- Center of Genomic and Post-Genomic, IRCCS Mondino Foundation, Pavia, Italy.

Acquired Brachial Plexopathy is a neurological disorder usually involving adults or children and often due to traumatic or compressive causes. 
In this regard, Idiopathic Neuralgic Amyotrophy (INA) rarely occurs and is characterized by sudden onset of sharp pain at the level of the shoulderand subsequent severe amyotrophy.

The incidence of INA is about 2-3/100.000 year in the whole population, with a mean onset age of 41,3 years. In childhood and adolescence, INA is much less frequent. Whether early-onset INA has a different phenotype and a better prognosis is still debated.

In a few cases mutations of SEPT9 gene were reported, whereas other cases have an unkown origin, possibly related to external trigger factors, such as infections, vaccinations, stressful conditions, surgery, or intense exercise. Literature about pediatric cases of INA is scarce.

Here, we present a case-series of three paediatric patients, recently diagnosed at the Neurology of Childhood and Adolescence Unit of Mondino Foundation in Pavia. We therefore describe their diagnostic work-up, including electromyographic assessment, imaging and molecular studies, and clinical follow-up

\section{Validation of the Italian version of the Charcot-Marie-Tooth Health Index (CMT-HI)}

C. Pisciotta ${ }^{1}$, E. Ciafaloni ${ }^{2}$, R. Zuccarino ${ }^{3,4}$, D. Calabrese ${ }^{1}$, P. Saveri ${ }^{1}, \mathrm{~S}$. Fenu $^{1}$, I. Tramacere ${ }^{1}$, F. Genovese ${ }^{5}$, N. Dilek ${ }^{2}$, N.E. Johnson ${ }^{6}$, C. Heatwole $^{3,7}$, D.N. Herrmann ${ }^{2}$, D. Pareyson ${ }^{1}$, on behalf of the ACTCMT study

${ }^{1}$ Fondazione IRCCS Istituto Neurologico Carlo Besta, Milan, Italy;

${ }^{2}$ Department of Neurology, University of Rochester, Rochester, NY, USA;

${ }^{3}$ Department of Neurology, University of Iowa Carver College of Medicine, Iowa City, IA, USA;

${ }^{4}$ Neuromuscular Omnicentre (NEMO)-Fondazione Serena Onlus, Arenzano, GE, Italy;

${ }^{5}$ ACMT-Rete per la Charcot-Marie-Tooth, OdV, Bologna, Italy;

${ }^{6}$ Department of Neurology, Virginia Commonwealth University (VCU), Richmond, VA, USA;

${ }^{7}$ Center for Health and Technology (CHET), University of Rochester, Rochester, NY, USA .

The CMT-HI is a disease-specific patient reported outcome measure (PROM) measuring overall disease burden in CMT patients (Johnson et al. Ann Neurol.2018;84:225-233), designed for natural history studies and clinical trials in English-speaking affected individuals. We developed and validated the Italian version of the CMT-HI (I-CMT-HI).

The questionnaire was translated and culturally adapted from source into Italian by two neurologists experienced in CMT and neuromuscular disorders (NMD). The two translations were reviewed by a panel of seven experts in CMT and NMD. The provisional version was back-translated into English by a professional translator. The definitive Italian version was developed during a consensus teleconference by the panel and a patient representative from ACMT-Rete. A series of well characterized CMT patients completed the final questionnaire; a subset had a second test administration after 1-4 weeks.

The I-CMT-HI was administered to 30 CMT patients (13 CMT1A, 8 CMTX1, 2 CMT1B, 2 CMT1E, 2 CMT2I, 1 CMT2A, 1 CMT2N, 1 $\mathrm{dHMN}$ ), with test-rest in 11: 14 females, 16 males, aged (mean $\pm \mathrm{SD}$ ) 48.0 \pm 16.4 years (range 18-81), with CMTES $=10.0 \pm 4.4$ (range 2-18). The ICMT-HI mean total score was 29.4 \pm 21.2 (range 0.1-60.3). The I-CMT-HI showed a high test-retest reliability: ICC $=0.95$ (95\% CI, 0.84-0.99). No patient had difficulty in completing the questionnaire; none reported problems with the questions' formulation. The total CMT-HI score was positively correlated with age and CMTES, with higher disease burden with increasing age and disease severity according to the CMTES.

The I-CMT-HI is now ready for use in clinical studies in the Italian population.
Charcot-Marie-Tooth disease and pregnancy: data from the italian CMT National Registry

C. Pisciotta ${ }^{1}$, D. Calabrese ${ }^{1}$, L. Santoro L ${ }^{2}$, I. Tramacere ${ }^{1}$, F. Manganelli ${ }^{2}$, GM. Fabrizi ${ }^{3}$, A. Schenone ${ }^{4}$, T. Cavallaro ${ }^{3}$, M. Grandis ${ }^{4}$, S. Previtali ${ }^{5}$, I. Allegri $^{6}$, L. Padua ${ }^{7}, 8$, C. Pazzaglia ${ }^{7}$, A. Quattrone ${ }^{9}$, P. Valentino ${ }^{9}$, S.

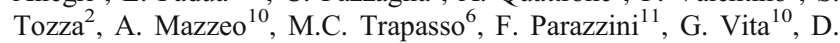
Pareyson $^{1}$; for the Italian CMT Network.

${ }^{1}$ Fondazione IRCCS Istituto Neurologico Carlo Besta, Milan;

${ }^{2}$ Federico II University, Department of Neurosciences, Reproductive Sciences and Odontostomatology, Naples;

${ }^{3}$ University of Verona, Department of Neurological, Biomedical and Motor Sciences, Verona;

${ }^{4}$ University of Genoa, Department of Neurosciences, Rehabilitation, Ophthalmology, Genetics and Maternal Infantile Sciences, Genoa;

5 Ospedale San Raffaele, Vita Salute San Raffaele University, Department of Neurology and INSPE, Milan;

${ }^{6}$ A.O. di Parma, U.O. Neurologia, Parma;

${ }^{7}$ Università Cattolica del Sacro Cuore, Rome;

${ }^{8}$ Don Carlo Gnocchi Onlus Foundation, Department of Neuroscience, Milan;

${ }^{9}$ Magna Graecia University, Department of Medical Sciences, Catanzaro;

${ }^{10}$ University of Messina, Unit of Neurology, Department of Clinical and Experimental Medicine, Messina; Italy;

${ }^{11}$ Department of Woman, Newborn and Child, Fondazione Istituto di Ricovero e Cura a Carattere Scientifico (IRCCS) Ca' Granda Ospedale Maggiore Policlinico, Milan; Italy.

Increased rate of pregnancy complications and occasional worsening of Charcot-Marie-Tooth (CMT) during pregnancy have been reported, but there are no large systematic studies.

Through an ad hoc online questionnaire, we investigated pregnancy and neuropathy course in CMT women adhering to the CMT Italian Registry. Controls were recruited among friends and unaffected relatives. Data were compared with the Italian (or other reference) population.

We collected data on 193 pregnancies from 86 CMT women (aged 20-73 yrs.), with 157 deliveries ( $81.4 \%$ ) after a mean of 38.6 gestational weeks. In CMT women there were no differences with controls $(n=24)$ and the reference population for: miscarriages (11.4\%), planned (21\%) and emergency $(13.3 \%) \mathrm{C}$-sections. We found however a significantly higher frequency of abnormal fetal presentations $(8.4 \%, \mathrm{p}=0.02)$ and preterm deliveries $(20.3 \%, \mathrm{p}<0.01$; most of them however in the $34-36$ week range) as compared to reference populations. Excluding twins, newborn weight $<2.5-\mathrm{Kg}$ rate $(7.3 \%)$ did not differ from the reference population. Postpartum bleeding rate in CMT patients $(n=3,2.1 \%)$ was similar to the general population (2.4\%). CMT status worsened during 18/193 pregnancies $(9.3 \%)$ with no recovery in 16 of them and similar figures in CMT1A vs non-CMT1A subtypes.

In conclusion, we observed slightly higher rate of abnormal presentations and preterm deliveries in CMT, but pregnancy outcome and newborn weight and health were similar to the reference populations. Worsening of CMT is not infrequent and occurs not only in CMT1A. Pregnant CMT women need to be monitored with particular care.

Supported by Telethon-Foundation GUP13006-grant.

Assessment of oxaliplatin-induced peripheral neurotoxicity in different mouse models

E. Pozzi ${ }^{1}$, A. Canta ${ }^{1}$, N. Oggioni ${ }^{1}$, M. Bossi ${ }^{1}$, G. Cavaletti ${ }^{1}$, P. Marmiroli $^{1}$ 
${ }^{1}$ Experimental Neurology Unit and Milan Center for Neuroscience, School of Medicine and Surgery, University of Milano-Bicocca, Monza, Italy

Oxaliplatin (OHP) is a third-generation platinum drug that can induce acute and chronic peripheral neurotoxicity (OIPN) in the clinical setting. In order to elucidate the pathogenesis associated with OIPN, several mouse models have been established. However, the characterization of this pathology is still unclear.

Using a multimodal approach, we assessed the OIPN onset in four different mouse models. Adult mice were treated with OHP treatment schedules very different from each other: 1) OHP $10 \mathrm{mg} / \mathrm{Kg}$, d1 e d3, i.p., C57BL $/ 6$; 2) OHP $3 \mathrm{mg} / \mathrm{Kg}$, single i.p., C57BL/6; 3) OHP 3mg/Kg, q1dx5, 2 cycles with 5 days rest, i.p., C57BL/6; 4) OHP $5 \mathrm{mg} / \mathrm{Kg}$, $2 q w x 4$, i.v., BALB/c. At different time-points, for each study we performed behavioral tests, neurophysiological and neuropathological analyses in order to monitor the development of OIPN.

In Study 2, we did not observed any features of OIPN. In Studies 1 and 3, we observed alterations mainly in cold sensitivity, in dorsal root ganglia (DRG) morphometry and, only in Study 1 , in nerve morphometry. The OHP schedule used in Study 4 induced acute cold hyperalgesia in addition to changes that may be associated with chronic OIPN (decrease in caudal and digital nerves sensory action potential amplitude, mechanical allodynia, alterations in DRG neurons and nerves morphometry, reduction in intraepidermal nerve fiber [IENF] density).

These results suggest that the OHP schedules used in Studies 1 and 3 can be useful to study neuropathic pain whereas the one used in Study 4 is the most suitable for mimicking the acute and chronic effects of OIPN

\section{Validation of a new Hand Function Outcome Measure in individuals with Charcot-Marie-Tooth}

Valeria Prada ${ }^{1}$, Mehrnaz Hamedani ${ }^{1}$, Giulia Robbiano ${ }^{1}$, Giulia Mennella $^{1}$, Alessandro Geroldi ${ }^{1}$, Angela Zuppa ${ }^{1}$, Sara Massucco ${ }^{1}$, Riccardo Zuccarino ${ }^{2}$, Laura Mori ${ }^{1,3}$, Emilia Bellone ${ }^{1}$, Paola Mandich ${ }^{1}$ ${ }^{, 2}$, Marina Grandis ${ }^{1,3}$, Angelo Schenone ${ }^{1,3}$

${ }^{1}$ DINOGMI, University of Genova, Genova, Italy,

${ }^{2}$ University of Iowa, Coralville, IA, United States

${ }^{3}$ Policlinico IRCCS San Martino, Genova, Italy

The symptomatology of Charcot-Marie-Tooth (CMT) disease mainly involves the feet and the hands. To date, there is no consensus on how to evaluate hand function in CMT. The aim of this study is to correlate the data of the engineered glove Hand Test System (HTS) with specific tests and the CMTES. We analyzed the hand of 44 patients with diagnosis of CMT using HTS, which measures the hand dexterity. Therefore, we completed the evaluation with the CMTES, Tripod Pinch and Hand Grip strength tested by a dynamometer, thumb opposition test (TOT) and Sollerman Hand Function Test (SHFT). TOT correlates with CMTES significantly in both hands $(p<0,0001)$. Tripod Pinch shows a statistically significant correlation with CMTES in the Dominant Hand $(\mathrm{DH})$ and Non Dominant Hand (NDH) (DH:p=0,002; NDH:p=0.005). Correlation between the hand grip of the DH and CMTES is significant (DH:p=0,002), in the NDH this parameter is not statistically significant. SHFT has a significant correlation with the CMTES (DH:p= 0,002$)$. HTS can be use with different tasks. HTS data correlates significantly with CMTES in both hands and in both tasks requested. There is no statistical correlation between the $\mathrm{DH} / \mathrm{NDH}$ ratio of all the outcome measures and CMTES, except for the hand grip $(\mathrm{p}=0,040)$. In conclusion, HTS parameters correlate with CMTES confirming that this tool is sensitive to the hand deficits. We can state that HTS is a good, simple to use and objective instrument to evaluate the hand of CMT patients, even if more studies about responsiveness and sensitivity are needed.
Definition of a new ICF core-set for the upper limbs of hereditary neuropathies

V. Prada ${ }^{1}$, B. Mazzarino ${ }^{2}$, A. Mazzeo ${ }^{3}$, D. Pareyson $^{4}$, L. Santoro ${ }^{5}$, L. Padua $^{6,7}$, GM. Fabrizi ${ }^{8}$, A. Schenone ${ }^{1,9}$ and ULNA group

${ }^{1}$ Department of Neurosciences, Rehabilitation, Ophthalmology, Genetic and Maternal and Infantile Sciences (DINOGMI), University of Genova, Genova, Italy

${ }^{2}$ SIALife s.c.r.l.

${ }^{3}$ Dipartimento di Medicina Clinica e Sperimentale, University of Messina, Messina, Italy

${ }^{4}$ Unità Malattie Neurodegenerative e Neurometaboliche Rare, Dipartimento Neuroscienze Cliniche, Fondazione IRCCS Istituto Neurologico Carlo Besta, Milan, Italy

5 Department of Neuroscience and Reproductive and Odontostomatological Sciences, University of Naples Federico II, Naples, Italy

${ }^{6}$ Unità Operativa di Neuroriabilitazione ad Alta Intensità, Fondazione Policlinico Universitario A. Gemelli IRCCS, Roma, Italy

${ }^{7}$ Dipartimento di Scienze Geriatriche e Ortopediche, Università Cattolica del Sacro Cuore, Roma

${ }^{8}$ Dipartimento di neuroscienze, biomedicina e movimento, Università di verona, Verona, Italy

${ }^{9}$ Policlinico San Martino IRCCS, Genova, Italy

${ }^{10}$ Casa di Cura del Policlinico, Milan, Italy

ULNA group: I. Poggi ${ }^{1}$, L. Mori ${ }^{1}$, M. Grandis ${ }^{1,}{ }^{9}$, C. Gemelli ${ }^{1}$, L.

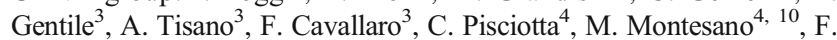

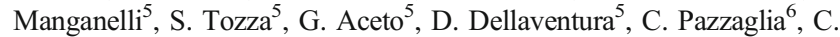

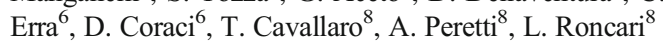

The International Classification of Functioning, Disability, and Health (ICF) is currently fundamental for health and functional status assessment, planning and monitoring treatments and outcome measurements. However, it has been scarcely applied to Charcot-Marie-Tooth (CMT) Neuropathy so, no standardized ICF Core-Set is available to define hand dysfunction in CMT. The definition of an ICF Brief Core-Set specific for the upper limbs (UL) in hereditary neuropathies will support medical care of patients allowing standardized reporting and measurement.

A panel of 25 health professionals ( 16 neurologists, 6 physiotherapists, 3 physiatrists) has been recruited to participate in an online Delphi survey to identify the properly ICF code. First step has been needed to choose the main chapters, in the phase 2 they selected the main codes and phase 3 individuated the codes of a Brief Core-Set, useful to describe the neuropathy disease at the UL level.

The brief core-set defined by the experts included the following codes: b260 Proprioceptive function, b289 Sensation of pain, other specified and unspecified, b7301 Strength of the muscles of a limb, b7351 Tone of the muscles of a limb, b7601 Control of complex voluntary movements, s7302 Structure of the hand, d170 Writing, d4402 Manipulate and d550 Eating.

In conclusion, this is the first ICF Brief Core-Set for the UL in hereditary neuropathies, created by a panel of experts thanks to an online Delphi survey. This could be a strong and useful tool to evaluate the patients. Next step will be the validation on the patients. Study funded by AFMgrant\#20821.

Long-term follow-up and clinical features in an Italian cohort of patients with GNE myopathy.

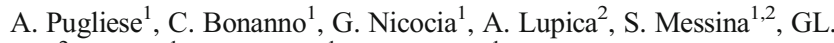

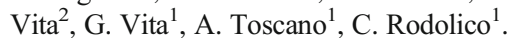


${ }^{1}$ Department of Clinical and Experimental Medicine, Unit of Neurology and Neuromuscular Diseases, University of Messina, Italy

${ }^{2}$ Nemo Sud Clinical Centre, Messina, Italy

Background: GNE myopathy is a muscle disease due to mutations in the $G N E$ gene, which encodes for the key enzyme of sialic acid biosynthesis. At disease onset, GNE patients present distal lower limb muscles weakness that slowly progresses with the involvement of other lower and upper extremities muscles.

Patients and methods: We describe a cohort of 13 patients ( $9 / 13$ women and 4/13 men) affected by GNE myopathy. Clinical and laboratory follow-up mean time was of 10 years. The diagnoses were based on clinical evaluation, EMG, muscle MRI/CT, muscle biopsy and genetic analysis.

Results: Our patients have an average age of 44 years old. The onset of symptoms was between the second and third decade of life. In the early stage, every patient complained difficulty in walking caused by foot drop. Since the onset of symptoms, 10 patients are now wheelchair-bound and quadriceps is typically spared in each patient, as commonly described for GNE myopathy. Only one patient developed respiratory failure requiring non-invasive ventilation. EMG study revealed a myopathic pattern in all patients. Muscle biopsy showed atrophic fibers with "rimmed" vacuoles. All our patients are genetically characterized: $4 / 13$ present a homozygous mutation in GNE gene, 9/13 a compound heterozygous one.

Conclusions: GNE myopathy is a very rare muscle disorder, probably under-diagnosed because of the large group of diseases manifesting with foot drop. Although that, it's necessary to suspect it in case of distal and bilateral lower limb muscles weakness with quadriceps sparing. Several aspects of this myopathy are still unclear and there is no approved therapy, but novel therapeutic strategies continue to be explored.

Clinical, laboratory and therapeutic follow-up of large cohort of Late Onset Pompe Disease (LOPD) patients: a single Centre experience.

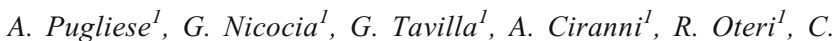

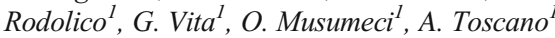

Department of Clinical and Experimental Medicine, Neurology and Neuromuscular Disorders Unit, University of Messina, Italy

Background: Pompe disease is a metabolic disorder, caused by deficiency of lysosomal acid $\alpha$-glucosidase (GAA) that is involved in glycogen degradation. It may occur in infantile and late onset (LOPD) forms. LOPD presents as a multisystem disease, mainly characterized by proximal limb-girdle myopathy with respiratory failure. Since 2006, an Enzyme Replacement Therapy (ERT) is available with lack of data on long-term efficacy.

Aim: To evaluate clinical, laboratory and therapeutic data in a large cohort of LOPD patients diagnosed and followed in our ERN-NMD Centre.

Patients and methods: In the last 30 years, we have diagnosed and followed up 46 LOPD patients by neurological, respiratory and muscle MRI studies, also with functional tests as 6MWT, GSGC and respiratory tests. Muscle biopsy and GAA assay activity in skeletal muscle were performed in 41 patients. Since 2008, we have treated 38/46 patients with ERT.

Results: $52 \%$ of patients manifested at onset proximal/ axial muscle weakness, 44\% hyperCKemia, myalgia and exercise intolerance, $4 \%$ some atypical features (i.e. eyelid ptosis, rigid spine). At the time of diagnosis, 43/46 patients presented elevated CK values (range 200$2000 \mathrm{UI} / 1)$. In 26/41 muscle biopsy revealed vacuolar myopathy with increased glycogen; GAA residual activity was markedly reduced (range $0.3 \%-30 \%$ ). Since 2008, 38 patients started ERT and have been followed for a variable period (follow up range 1-12 years). During the course of ERT, we registered a significant worsening at $6 \mathrm{MWT}$ in $46 \%$ of patients, while $21 \%$ improved in motor performances and $32 \%$ remained stable. We observed worsening in respiratory function in $50 \%$ of patients. Conclusions: Our study confirms that in LOPD patients clinical presentations and laboratory results are heterogeneous. Long-term results on treated patients evidenced variable responses to therapy, showing in some patients stability or positive results but overtime almost $50 \%$ of the patients show a deterioration of muscle and respiratory parameters.

Anti-AChR Myasthenia Gravis presenting with early predominant left triceps weakness, associated with reversible muscular atrophy.

\author{
A. Rasera ${ }^{1}$, M. Barillari ${ }^{2}$, D. Cavalli ${ }^{2}$, S. Romito ${ }^{1}$ \\ ${ }^{1}$ Neurologia A, AOUI Verona \\ ${ }^{2}$ Radiologia, AOUI Verona
}

Myasthenia gravis (MG) is a neuromuscular transmission disease whose usually presentation consists in fluctuating weakness of skeletal muscles. It can be generalized or focal, the latter mainly involving ocular muscles, but unusual presentations are described. In literature, only 14 cases of focal triceps weakness as MG onset symptom are reported, with AfroAmerican predominance. Muscular atrophy is also an uncommon finding in clinical practice that is associated to moderate-high dose corticosteroid treatments, anti-MUSK antibodies presence or to long-standing disease. In extremely rare cases, muscular atrophy can be present as first manifestation of MG.

We describe a Caucasian male patient whose onset symptoms were nonfluctuating weakness in left elbow extension, right forced grip with left triceps muscle and right forearm wasting and subsequently left eyelid ptosis. Electromyography discovered rare myopathic MUAPs in his left triceps and upper limbs magnetic resonance imaging (MRI) displayed right flexor digitorum profundus and bilateral triceps muscular bulk reduction.

Clinical manifestations, serological and electro-diagnostic tests were suggestive of anti-AChR MG. Corticosteroids therapy was initiated with improvement of muscular strength and electromyography recruitment pattern. Intriguingly, MRI performed 1 year after the first one showed increased flexor digitorum profundus and triceps muscles volume and recovery from fatty involution where previously described.

Our case report highlights the importance of recognizing atypical MG presentations, such as focal muscles (particularly triceps) weakness. Our work draws special attention to the fact that selective muscles atrophy may be a MG onset symptom more frequently than reported and that it can be reversible with treatment.

Early neurophysiological abnormalities in Guillain-Barré Syndrome: 4-year experience of Verona center

A. Rasera ${ }^{1}$, S. Romito ${ }^{1}$, A. Segatti ${ }^{1}$, E. Concon ${ }^{1}$, L. Alessandrini ${ }^{1}$, F. Basaldella ${ }^{1}$, A. Badari ${ }^{1}$, C. Arcaro ${ }^{1}$, B. Bonetti ${ }^{1}$, G. Squintani ${ }^{1}$.

${ }^{1}$ Neurologia A, AOUI Verona, Italy

Guillain Barré syndrome (GBS) is an acute-onset disorder of the peripheral nervous system that may be difficult to identify in the initial stages because diagnostic criteria are not always fulfilled. Our aim is to identify the most common electrophysiological abnormalities in early neurophysiological evaluations of patients affected by GBS.

We reviewed clinical documents of all patients with confirmed diagnosis of GBS, admitted to our Neurology unit in the last 4 years. We included patients who underwent nerve conduction studies (NCS) within 15 days from symptoms onset. Our cohort was divided in two subgroups according to the interval between disease onset and NCS: "very early" (within 7 days) and "early" (7-15 days). We evaluated several neurophysiological 
factors. 32 patients fulfilled the diagnostic criteria of GBS: 17 patients belonged to the "very early" subgroup, the other 15 patients were considered as "early" subset. The most sensitive electrophysiological was the bilateral absence of tibial nerve $\mathrm{H}$ reflex, followed by $\mathrm{F}$ wave abnormalities. Demyelinating features in motor nerve conduction were not so common as well as cMAP amplitude decrease. In all subgroups, sensory conductions abnormalities were found in less than $40 \%$ in all subgroups.

Our findings confirm that proximal segments are the most frequently affected in the early phases of the disease and strengthen the importance of an exhaustive NCS, including $\mathrm{H}$ reflex and $\mathrm{F}$ waves evaluations, in the diagnosis of GBS.

\section{Proposal of new functional motor scales to evaluate muscle fatigue in adult SMA patients}

G. Ricci ${ }^{1}$, A. Govoni ${ }^{1,2}$, I. Bortone ${ }^{3}$, L. Billeci ${ }^{4}$, A. Borelli ${ }^{1}$, L. Manca ${ }^{1}$,

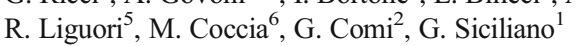

${ }^{1}$ Neurological Clinics, Department of Clinical and Experimental Medicine, University of Pisa, Pisa

${ }^{2}$ Neurology Unit, Fondazione IRCCS Ca' Granda, Ospedale Maggiore Policlinico, Milan

${ }^{3}$ School of Medicine, Department of Basic Medical Sciences, Neuroscience and Sense Organs (DSMBNOS), University of Bari "Aldo Moro", Bari

${ }^{4}$ National Research Council of Italy (CNR), Institute of Clinical Physiology (IFC), Pisa

${ }^{5}$ Department of Biomedical and NeuroMotor Sciences, University of Bologna, Bologna

${ }^{6}$ Neurorehabilitation Clinic, United Hospitals of Ancona, Ancona

The advent of Nusinersen as therapy for spinal muscular atrophy (SMA) represented the beginning of the modification of the pathology course. It therefore becomes essential to find outcome measures that can demonstrate its effectiveness, especially in adult patients with a long disease duration. Patients with SMA easily fatigue during repetitive activities of daily living. Since outcome measures sensitive to change in fatigability are lacking, their development is a pivotal step.

We present a new functional scale designed to evaluate the exhaustion of the upper and lower limbs according to the patient's motor abilities. The items for upper limbs are: Endurance in keeping up a weight, Exhaustible exercise of proximal upper limbs with repetitive tasks, Hand-Grip endurance with repetitive maximal isometric contraction, Repeated nine-hole peg test, Repetitive open and close hands with the elbows resting, Digital dexterity repetitive test, and for lower limbs: 6-minute-walking test, 5 Sitto-Stand test, and Repetitive timed-up-and-go test.

Moreover, in an experimental setting, we tested a technology-based protocol that integrates functional data from wireless surface electromyography probes (BTS FREEEMG, BTS SpA, Italy) and wireless inertial sensors (G-SENSOR 2, BTS SpA, Italy) in order to analyze muscle strength and fatigue during specific motor tasks.

The recent trends in neuromuscular studies indicate that new scenarios are being proposed in terms of both therapeutic possibilities and clinical experimentation. The validation of the proposed scales will provide new perspectives in terms of follow up and outcome measures; the use of wearable devices can provide more reliable, repeatable and sensitive data.

Neuromuscular features in Chorea-Acanthocytosis: a clinical and histopathological report

B. Risi ${ }^{1}$, S. Cotti Piccinelli ${ }^{1}$, F. Novara ${ }^{2}$, E. Baldelli ${ }^{1}$, N. Necchini ${ }^{1}$, A.

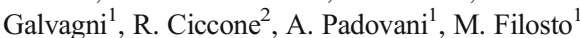

${ }^{l}$ Center for Neuromuscular Diseases, Unit of Neurology, ASST Spedali Civili and University of Brescia, Brescia, Italy

${ }^{2}$ Microgenomics Laboratory, Pavia, Italy

Chorea-acanthocytosis $(\mathrm{ChAc})$ is a very rare autosomal recessive adultonset neurodegenerative disorder caused by mutations in VPS13A, a gene that directs structural proteins in red blood cells.

Core clinical features are choreiform movements affecting both trunk and extremities, orolingual dyskinesia, parkinsonism, epilepsy, behavior changes and acanthocytosis in peripheral blood smear. Some ChAc patients may develop mild clinical neuromuscular manifestations including elevated levels of creatine phosphokinase, distal muscle atrophy and weakness and areflexia.

We report a 41-year-old patient which complained of progressive gait impairment and dysarthria since age 38 . In the past clinical history epilepsy since age 14 and drug abuse were reported.

Neurological examination showed primarily signs of neuromuscular involvement as: impaired walking on heels, weakness of thigh flexor and foot dorsal and plantar flexor muscles, upper limb-girdle and hand muscle atrophy, absent ankle jerk reflexes, hypophonia, dysarthria and dysphagia. Milder extrapyramidal signs were also noted i.e. axial and lower limb plastic rigidity, hypokinesia and hypomimia.

EMG-ENG showed widespread chronic neurogenic changes. Muscle biopsy displayed a marked non-specific myopathic pattern (variability of fiber diameters; scattered atrophic fibers; many fibers with internal nuclei; fiber splitting; one fiber necrosis; some ring and whorled fibers; rare fibers with subsarcolemmal accumulations of mitochondria and one ragged red fiber) and less prominent neurogenic changes (small fiber type grouping).

Brain MRI showed atrophy and signal alterations in lenticular and caudate nuclei.

Genetic study detected the homozygote frameshift mutation c.1589 1595 +5 del in exon 17 of the VPS13A gene involving the splicing donor site (NP 150648.2: p.Gln529Hisfs *6).

Our report shows that ChAc may present with prevalent neuromuscular clinical features due to primary myopathy and/or axonal/motor neuron chronic loss. The association with extrapyramidal signs and epilepsy should induce the diagnostic suspicion, even in absence of significant involuntary movements.

Benign monomelic amyotrophy of upper limb (Hirayama disease): a single center analysis

MG Rispoli ${ }^{1}$, L. Ferri ${ }^{1}$, M. Di Pietro ${ }^{1}$, V. Di Stefano ${ }^{2}$, A. Di Muzio ${ }^{3}$.

${ }^{1}$ Department of Neuroscience Imaging and Clinical Sciences, Gabriele d'Annunzio University of Chieti and Pescara, Chieti, Abruzzo, Italy;

${ }^{2}$ Department of Biomedicine, Neuroscience and advanced Diagnostic, University of Palermo, Palermo, Sicily, Italy;

${ }^{3}$ Center for Neuromuscular Diseases, "SS Annunziata" Hospital, Chieti, Abruzzo, Italy.

Hirayama Disease (HD), a very rare sporadic distal amyotrophy of the upper limbs, characterized by insidious onset and a self-limited course, affects mainly young males and is more common in the oriental countries $^{1}$. Few italian cases have been reported ${ }^{2}$. The involvement is exclusively motor, mainly of the right side and there are no clinical or laboratory pathognomonic findings. MRI of cervical spine in flexion shows a particular aspect considered important for the diagnosis ${ }^{3}$.

Methods: we reviewed data of 8 patients with HD from Abruzzo (4F, range 26-65year-old), afferent to the Neuromuscular Center of Chieti in the last 10 years. We've collected a detailed history and evaluated them by using electrophysiology and cervical spine MRI in flexion position. The course is 2-27 years (mean 16,1years). They have been ruled out all other possible disorders. 
Results: Some peculiarities came out of the studio. In 4/6 (66.7\%) of patients MRI did not show the characteristic findings. In $3 / 8(37.5 \%)$ the pathology has a very slowly progressive course. In $7 / 8(87.5 \%)$ it is the left side that is mainly or exclusively involved. No patient reported traumatism or particular physical activities before the onset of the disease. Conclusion: in our patients the diagnosis of HD remains fundamentally clinical-anamnestic with electrophysiological support. The disease has no clear correlation with physical or other activity and in some cases show a slowly progressive course.

1. Zhou B. et al. Amyotroph Lateral Scler. 2010;11(1-2):133-9.

2. Vitale V. et al. QuantImagingMedSurg 2016;6(4):364-37.

3. Lai V. et al. EurJRadiol 2011;80:724-28.

Systemic Gene Transfer With rAAVrh74.MHCK7.SGCB Increased $\beta$-Sarcoglycan Expression in Patients With Limb Girdle Muscular Dystrophy Type 2E

LR. Rodino-Klapac ${ }^{1,3}$, ER. Pozsgai ${ }^{1,3}$, S. Lewis ${ }^{1,3}$, DA. Griffin ${ }^{1,3}$, AS. Meadows ${ }^{1,3}$, L. Picaro ${ }^{3}$, KJ. Lehman ${ }^{1}$, K. Church ${ }^{1}$, NF. Miller ${ }^{1}$, MA. Iammarino $^{1}$, LP. Lowes ${ }^{1}$, JR. Mendell ${ }^{1,2}$

${ }^{1}$ Center for Gene Therapy, The Abigail Wexner Research Institute at Nationwide Children's Hospital, Columbus, Ohio, USA

${ }^{2}$ Department of Pediatrics and Neurology, The Ohio State University, Columbus, OH, USA

${ }^{3}$ Sarepta Therapeutics, Inc., Cambridge, MA, USA

Background: Limb girdle muscular dystrophies (LGMD) manifest with progressive hip/shoulder muscle weakness. LGMD2E ( $\beta$-sarcoglycan [SGCB] deficiency) includes cardiac involvement and elevated creatine kinase (CK).

Material and Method: Initial findings of ongoing, phase 1 multiple ascending-dose gene transfer trial of $\leq 9$ patients with LGMD2E who received rAAVrh74.MHCK7.SGCB (NCT03652259). Participants: patients $4-15 \mathrm{y}$, confirmed SGCB mutation (both alleles), negative for rAAVrh74 antibodies, $>40 \%$ n on 100 -meter timed test. Patients received single IV infusion of $5 \times 10^{13} \mathrm{vg} / \mathrm{kg}$ rAAVrh74.MHCK7.SGCB. Prednisone $1 \mathrm{mg} / \mathrm{kg}$ /day was initiated 1 day before gene delivery, tapering after 30 days. Primary endpoints: $\geq 20 \%$ SGCB-positive fibers (Day60 muscle biopsy) and safety (Day 270). Secondary endpoints: CK decrease and functional endpoints (Day 270).

Results: First 3 patients enrolled (age 13, $n=2$; age 4 ; $n=1$ ): robust SGCB expression observed by immunohistochemistry (IHC), with a mean of $51 \%$ SGCB positive fibers (range $42-63 \%$ ) expressing a mean $47 \%$ intensity (range $38-57 \%$ ). Co-localization of $\alpha$-sarcoglycan observed (IHC). Western blot showed a mean 36.1\% SGCB expression vs normal (range 34-39\%). Mean CK levels reduced by $82 \%$, suggesting slowed muscle destruction. Two patients had elevated liver enzymes (1 serious) and 1 had elevated bilirubin following oral steroid taper, which returned to baseline. Two patients had transient mild nausea, corresponding with increased steroid dosing. No other clinically significant lab findings.

Conclusion: Gene transfer in patients with LGMD2E following rAAVrh74.MHCK7.SGCB infusion was positive for defined endpoints. This is the second gene therapy inducing protein production post transgene delivery with rAAVrh74 vector and MHCK 7 promoter, demonstrating potential benefits of a rationally designed delivery system.

\section{A case of intraneurial perineurioma. Limits and advantages of imag- ing techniques.}

M. Romano ${ }^{1}$, D. Coraci $^{2}$, S. Cottone ${ }^{3}$, R. Gasparotti ${ }^{4}$, S. Ferraresi ${ }^{5}$, S. Realmuto ${ }^{1}$, E. Cammarata ${ }^{1}$, L. Padua ${ }^{2,6}$
${ }^{1}$ Neurology Unit, Azienda Ospedaliera Ospedali Riuniti Villa Sofia Cervello, Palermo, Italy.

${ }^{2}$ Neuroriabilitazione ad Alta Intensità, Fondazione Policlinico Universitario A. Gemelli IRCCS, Rome, Italy

${ }^{3}$ Neurology Unit, Ospedale Civico, Palermo, Italy

${ }^{4}$ U.O. Neuroradiologia, ASST Spedali Civili, Università degli Studi di Brescia, Brescia, Italy

${ }^{5}$ Department of Neurosurgery, Hospital S. Maria della Misericordia, Rovigo, Italy

${ }^{6}$ Università Cattolica del Sacro Cuore, Milan, Italy

Nerve ultrasound (US) is able to directly visualize, with no side effects, morphological changes of a compromised nerve. However, ultrasound presents some limitations. In particular, the bone is an almost absolute obstacle for the visualization of the body structures. The limitation due to the clavicle to assess a portion of brachial plexus is an emblematic situation. For this reason, where US is not able to get, magnetic resonance (MR) may be a valid completion. We present a case of a 39-year-old woman with 5-year history of progressive deficit in the left finger extension, with no sensory disturbances. Electrophysiological examination revealed fibrillation potentials and neurogenic reduced recruitment in the left extensor digitorum muscles. A mild reduction of sensory axonal potential was found. US of radial nerve along its course and brachial plexus did not reveal any alterations. Differential diagnosis in similar cases may present some difficulties. After this evaluation, the patient was assessed by the use of neuro-MR, in order to visualize the nerve in those tracts inaccessible with US. This examination revealed a fusiform lesion of the posterior cord of the brachial plexus at the infraclavicular level, presenting contrast enhancement after gadolinium administration. The lesion was surgically removed with a good clinical evolution. Hystopatological examination confirmed the diagnosis of perineurioma. The case shows the importance of an extensive evaluation in atypical cases. Each techniques has its peculiarities and limitations. When possible, the association of different approaches and different point of view can result in a large benefit for the patient.

TYMP mutations result in late onset mitochondrial myopathy with altered muscle mtDNA homeostasis

D. Ronchi ${ }^{1}$, L. Caporali ${ }^{2}$, G. Manenti ${ }^{1}$, M. Meneri ${ }^{3}$, S. Mohamed ${ }^{2}$, M. Contin $^{2}$, D. Piga ${ }^{3}$, V. Mancinelli ${ }^{1}$, S. Corti ${ }^{1,3}$, M. Sciacco ${ }^{4}$, V. Carelli ${ }^{2,5}$, G.P. Comi ${ }^{1,4}$.

1 Dino Ferrari Center, Department of Pathophysiology and Transplantation, University of Milan, Milan, Italy.

${ }^{2}$ Istituto delle Scienze Neurologiche di Bologna, UOC Clinica Neurologica, Bologna, Italy.

${ }^{3}$ Fondazione IRCCS Ca' Granda Ospedale Maggiore Policlinico, Neurology Unit, Milan, Italy.

${ }^{4}$ Fondazione IRCCS Ca' Granda Ospedale Maggiore Policlinico, Neuromuscular and Rare Diseases Unit, Milan, Italy.

${ }^{5}$ Dipartimento di Scienze Biomediche e Neuromotorie (DIBINEM), Università di Bologna, Bologna, Italy.

Mitochondrial NeuroGastroIntestinal Encephalomyopathy (MNGIE) is a neurological disorder featuring extraocular muscle weakness, gastrointestinal dysmotility, cachexia, sensorimotor peripheral neuropathy and leukoencephalopathy due to biallelic mutations in TYMP, encoding the thymidine phosphorylase (TP) enzyme. Due to the toxic accumulation of nucleoside in plasma, skeletal muscle displays altered mtDNA maintenance (mtDNA depletion/deletions) and abnormalities compatible with mitochondrial dysfunction. 
The vast majority of cases ( $>97 \%)$ exhibits an onset before the age of 20 years with a progressive course leading to a fatal outcome (classical form). A late onset (beyond 40 years of age) presentation has been described in four patients presenting multisystemic involvement and a slower progression.

Gene panel sequencing in a cohort of 60 patients featuring muscle accumulation of mitochondrial DNA (mtDNA) deletions detected TYMP defects in three subjects (5\%), two of them with symptom onset in the fifth decade. One of the patients only displayed ptosis and ophthalmoparesis.

In all of them muscle biopsy showed COX-negative/SDH positive fibers. Quantitative studies documented multiple deletions (but not depletion) of mtDNA, whereas muscle mtDNA of classical MNGIE patients displayed significant depletion and few deletions. Levels of residual TP activity in leukocytes and nucleosides in plasma suggest the existence of a threshold discriminating late onset presentations with isolated myopathy and multisystemic involvement.

Screening for TYMP mutations is recommended in adult patients with muscle mtDNA deletions, even in absence of cardinal MNGIE features.

Six muscular MRI patterns in search for an author: clinical, genetic and imaging characterization of six myopathic/dystrophic case reports without a definite diagnosis

M. Rossi ${ }^{1}$, M. Paoletti ${ }^{2}$, V. Vacchini ${ }^{1}$, A. Ferrero ${ }^{1,3}$, S. Parravicini ${ }^{1,3}$, A. Gardani ${ }^{1}$, A. Asaro ${ }^{4}$, A. Pichiecchio ${ }^{2,3}$, A. Berardinelli ${ }^{1}$.

${ }^{1}$ Child Neurology and Psychiatry Unit, IRCCS Mondino Foundation, Pavia, Italy

${ }^{2}$ Neuroradiology Unit, IRCCS Mondino Foundation, Pavia, Italy.

${ }^{3}$ Department of Brain and Behavioural Sciences, Unit of Child Neurology and Psychiatry, University of Pavia, Italy

${ }^{4}$ Genomic and Post-Genomic Center, IRCCS Mondino Foundation, Pavia, Italy.

In last years, increasing interest has emerged for a clear characterization of natural history of neuromuscular disorders. Given the increasing availability of therapeutic trials, a proper diagnostic characterization of the population based on clinical and molecular data has become imperative in order to be able to select patients and assess, with accuracy, the efficacy of such treatments. In the field of neuromuscular imaging, magnetic resonance (MRI) has proven to be a valid and non-invasive technique that is highly useful in determining the selective involvement of muscles and nerves, to suggest diagnosis especially when a clinical overlap of symptoms is present, but also to direct further diagnostic tests and to monitor the evolution of the disease.

We started from the revision of the population admitted to our Child Neurology and Psychiatry Unit with a clear clinical suspect of neuromuscular disorder in order to make an association study between clinical data, imaging e molecular genetics obtained with next-generation sequencing (NGS) techniques. The study was aimed at increasing the percentage of cases with a molecular definition, to find out new genes responsible for disease and to correlate the radiological genotype and phenotype.

We describe here six different pediatric cases with a strong suspicion for neuromuscular disorders for which we could identify a clear selective involvement at muscular MRI addressing further molecular analysis according to selective pattern described in literature. Anyway for these patients, despite the clinical, bioptic and molecular analysis and revision carried out, the diagnostic definition remains still unsolved.
Whole Exome Sequencing identifies two novel candidate genes and extends the diagnostic spectrum of patients with neuromuscular diseases

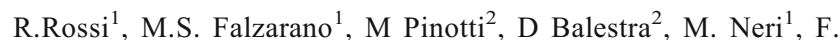
Fortunato ${ }^{1}$, E. Mercuri ${ }^{3}$, M. Pane ${ }^{3}$, F. Gualandi ${ }^{1}$, R. Selvatici ${ }^{1}$, A. Ferlini $^{1}$

${ }^{1}$ Unit of Medical Genetics, Department of Medical Sciences, University of Ferrara, Italy

${ }^{2}$ Section of Biochemistry and Molecular Biology, Department of Life Sciences and Biotechnology, University of Ferrara, Italy

${ }^{3}$ Unit of Child Neuropsychiatry of the Policlinico Gemelli, Catholic University of the Sacred Heart of Rome, Italy

We studied two families by WES (SOLVE-RD-EU project). The first family-of-four consists of two affected male siblings with Hereditary spastic paraplegia (HSP), the second was a family trio including healthy parents and a daughter with progressive ataxia and spastic gait. Prioritization for recessive model identified novel biallelic variations.

In the family-of-four, we found biallelic variations in 3 genes: PCLO, SARS2 and PNPT1. PCLO and SARS genes were excluded since respectively unmatched phenotype and unlikely pathogenic nature of the variations. In silico prediction of the biallelic variants PNPT1 showed that missense p.Ala507Ser variant (MAF $=0.000223$ ) of maternal origin and nonsense p.Tyr432* $(\mathrm{MAF}=0.0000)$ variant of paternal origin were predicted to be pathogenic. PNPT1 gene encodes an enzyme implicated in RNA-processing and known mutations are associated with COXPD13 and (OMIM\#614932) and DFNA70 (OMIM\#614934). Interestingly one patient presents hearing involvement and we hypothesize that HSP might be a new phenotype associated with PNPT1 mutations.

In the family trio, we found biallelic variations in the CPSF3L gene: a frameshifting variant p.Ala319CysfsTer2 $(\mathrm{MAF}=0.0001)$ and an intronic deletion c.1059+7_1059+17delGCATGGTGAGG (MAF=0.00003) predicted respectively to be pathogenic and to induce potential alteration of splicing. The latter was studied by a splicing reporter minigene-assay, confirming aberrant transcript accounts for $4 \%$ of all transcripts. CPSF3L, also known as INTS11, is part of a multiprotein complex called Integrator Complex. INTS11 is critical to ensure proper Uridylate-rich-small-nuclear-RNAs biosynthesis. We suggest CPSF3L as new candidate gene not previously associated with Ataxia and Spastic paraplegia. Zebrafish mutant studies are in progress for both candidate genes.

Evaluation of muscle involvement in Acromegaly and Cushing's syndrome

L. Ruggiero ${ }^{1}$, M.C. De Martino ${ }^{2}$, E. Spina ${ }^{1}$, R. Dubbioso ${ }^{1}$, S. Tozza ${ }^{1}$, C. Pivonello $^{2}$, C. Simeoli ${ }^{2}$, R. Ferrigno ${ }^{2}$, N. Di Paola ${ }^{2}$, R. Iodice ${ }^{1}$, L. Santoro $^{1}$, R. Pivonello ${ }^{2}$, F. Manganelli ${ }^{1}$

${ }^{1}$ Department of Neurosciences, Odontostomatology and Reproductive Sciences, University of Naples "Federico II", Italy

${ }^{2}$ Department of Clinical Medicine and Surgery, University of Naples Federico II, Italy

Muscle weakness is a common complaint of patients with Acromegaly or Cushing's syndrome. Though anecdotal reports of myopathy have been reported, muscular involvement in such endocrine disorders has never been investigated systematically.

Thus, 16 patients with Acromegaly ( 9 males) and 21 patients with Cushing's syndrome (4 males) were systematically evaluated, between 2018 and 2019, by a neurologist and an endocrinologist at University Federico II of Naples. 
All patients underwent laboratory evaluation (i.e. hormonal profile and $\mathrm{CK}$ ) and extensive clinical and functional assessment including the MRC sum score, the 6-minute walk test (6MWT), the 10meter walk test (10MWT), the 4-climb stair test (4CST; ascendant/descendant) and the Gowers' sign time (GT). All clinical data were compared with 32 healthy age-matched subjects. All patients underwent EMG examination and nerve conduction studies, 25 of them muscle MRI (16 Cushing's syndrome) and 3 patients (Cushing's syndrome) muscle biopsy.

Serum CK was slightly increased $(<300 \mathrm{U} / \mathrm{I})$ in $8 \%$ of patients. The 6MWT showed that patients with Cushing's syndrome walked on average less meters $(417 \mathrm{~m})$ than acromegalic $(499 \mathrm{~m} ; \mathrm{p}=0.002)$ and control (502 $\mathrm{m} ; \mathrm{p}=0.0001)$ groups while the 6MWT was not different between Acromegaly and controls. No difference was found for all the other clinical and functional assessments. Electrophysiological examination, muscle MRI and muscle biopsy did not reveal any significant myopathic alteration.

These preliminary results do not support a structural involvement of skeletal muscle in Acromegaly and Cushing's syndrome. However further investigations are needed.

\section{Description of the first cohort of V122I ATTRv amyloidosis patients from non-endemic areas}

M. Russo ${ }^{1}$, L. Gentile ${ }^{1}$, G. Di Bella ${ }^{2}$, F. Minutoli ${ }^{3}$, F. Cucinotta ${ }^{1}$, L.

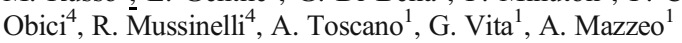

${ }^{1}$ Unit of Neurology and Neuromuscular Diseases, Department of Clinical and Experimental Medicine, University of Messina, Italy

${ }^{2}$ Department of Clinical and Experimental Medicine, University of Messina, Italy

${ }^{3}$ Department of Biomedical and Dental Sciences and Morphofunctional Imaging, University of Messina, Italy.

${ }^{4}$ Amyloidosis Research and Treatment Centre, Fondazione IRCCS Policlinico San Matteo, Pavia, Italy.

V122I is one of more than 130 mutations in transthyretin gene associated with hereditary TTR (ATTRv, v per variant) amyloidosis. Main clinical expression is an infiltrative pseudohypertrophic cardiomyopathy with mild or no neurological symptoms. It is particularly common among African Americans, with mutation prevalence between 3 and $4 \%$.

We report here 12 subjects from seven apparently unrelated Caucasian families hailing from Sicily and carrying the V122I mutation. One patient was homozygous for V122I and in another family two subjects also carried the E89Q variant in compound heterozygosity. All the subjects underwent neurologic/neurophysiologic evaluation and cardiologic baseline tests; in five of them, cardiac magnetic resonance and/or (99 m) Tc-DPD scintigraphy were performed.

$3 / 12$ subjects were asymptomatic carriers. Of the remaining nine subjects, in eight of them we found a classical cardiac phenotype, with a hypertrophic restrictive cardiomyopathy and cardiac failure (NYHA class II-IV), and a monolateral or bilateral carpal tunnel syndrome. In four patients, the nerve conduction studies revealed a polyneuropathy; in one of them, this represent the only sign of disease, with cardiologic tests still negative after five years of follow up.

Our report describes the first cohort of V122I patients from South Italy confirming the possible underestimation of this mutation in the nonAfrican population. Moreover, it highlights the heterogeneity in the genotype-phenotype correlation of ATTRv mutations, suggesting that the presence of a polyneuropathy has to be identified as soon as possible, since available treatments are so far authorized only for ATTRv amyloid peripheral neuropathy.
Whole genome sequencing in a pair of siblings affected with Duchenne muscular dystrophy with discordant cognitive phenotype

D. Sabbatini ${ }^{1}$, M. Alexander ${ }^{2}, \mathrm{~S}$. Vianello $^{1}$, A. Fusto ${ }^{1}$, B. Merlo ${ }^{1}$, M. Villa $^{1}$, V. Zangaro ${ }^{1}$, F. De Pascale ${ }^{3}$, G. Sorarù ${ }^{1}$, E. Pegoraro ${ }^{1}$, L. Bello ${ }^{1}$

${ }^{I}$ Neuromuscular Center, Department of Neurosciences DNS, University of Padova, Padova, Italy.

${ }^{2}$ Department of Pediatrics, University of Alabama at Birmingham, Birmingham, AL, U.S.A.

${ }^{3}$ Department of Biology, University of Padova, Padova, Italy.

Background: Central nervous system (CNS) involvement in dystrophinopathies is variable, although loosely associated with mutations in the C-terminal domains of dystrophin. We aimed to identify candidate variants involved in the modulation of CNS phenotype in dystrophinopathies, by performing whole genome sequencing (WGS) in a pair of Duchenne muscular dystrophy (DMD) siblings with discordant cognitive phenotype.

Materials (patients): Patient 1 was born of healthy, non-consanguineous parents. He was diagnosed with DMD at the age of 4 years (deletion $D M D$ exons 45-52), and lost ambulation at the age of 11 . He had no intellectual disabilities. Patient 2, his younger brother, was diagnosed with DMD due to the same mutation, and lost ambulation at the age of 12. However, he presented an autism-spectrum disorder. We performed WGS to analyse Structural Variants with Breakdancer algorithm ${ }^{1}$, while short indels and SNPs (single nucleotide polymorphisms) were filtered exploiting our own Python scripts.

Results: The algorithm filtered a "short list" of 41 variants, reviewed individually for potential pathogenetic mechanisms. The most notable variants were rs112339619, upstream of the ASD risk gene ANK3, previously reported in a WGS study ${ }^{2}$; and rs117696080, an intronic variant in NRXN3, encoding neurexin 3, a dystrophin-associated glycoprotein, and also identified in the same study. Patient 2 was heterozygous for both these variants, while patient 1 had a wild-type genotype at both loci.

Conclusions: Identified variants represent putative modifiers of the cognitive phenotype of DMD. We plan to validate these findings by sequencing a larger cohort of dystrophinopathy patients with and without cognitive issues.

1. Fan X, Abbott TE, Larson D, Chen K. BreakDancer: Identification of genomic structural variation from paired-end read mapping. Curr Protoc Bioinforma. 2014;

2. McKenna B, Koomar T, Vervier K, Kremsreiter J, Michaelson JJ. Whole-genome sequencing in a family with twin boys with autism and intellectual disability suggests multimodal polygenic risk. Cold Spring Harb Mol Case Stud. 2018;4.

Capturing the patient-reported impact of myasthenia gravis in the real-world setting using a smartphone application

F. Saccà ${ }^{1}$, S. Berrih-Aknin ${ }^{2}$, K. G. Claeys ${ }^{3,4}$, R. Mantegazza ${ }^{5}$, H. Murai ${ }^{6}$,

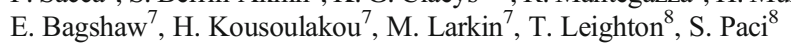

${ }^{1}$ Department of Neuroscience and Reproductive and Odontostomatological Sciences, University of Naples Federico II, Naples, Italy

${ }^{2}$ Center of Research in Myology, Sorbonne Université, INSERM, Institute of Myology, Paris, France

${ }^{3}$ Department of Neurology, University Hospitals Leuven, Leuven, Belgium

${ }^{4}$ Laboratory for Muscle Diseases and Neuropathies, Department of Neurosciences, KU Leuven, Leuven, Belgium

5 Department of Neuroimmunology and Neuromuscular Diseases, Fondazione I.R.C.C.S. Istituto Neurologico Carlo Besta, Milan, Italy

${ }^{6}$ Department of Neurology, International University of Health and Welfare, Narita, Japan 
${ }^{7}$ Vitaccess Ltd, Oxford, United Kingdom

8 argenx $B V$, Ghent, Belgium

Background: Myasthenia gravis (MG) is a chronic autoimmune disease, causing debilitating muscle weakness. Patient-reported data are crucial to improve knowledge about the burden of this condition and patients' unmet needs.

Aims: To design a patient-centric real-world study to allow people with MG to provide data about disease burden using an application on their smartphones.

Methods: The study methodology and application were co-developed with patients, patient advocacy groups (PAGs), and disease experts (neurologists, researchers), who also reviewed and validated the protocol. Health economists and statisticians were also consulted.

Results: We will conduct a two-year, prospective, observational study of $\sim 2,000$ adults with MG, resident in the US, Japan, Germany, France, the UK, Italy, Spain, Canada, and Belgium. Participants will use the application to check eligibility, provide consent and contribute data. They will complete a personal profile about demographics, MG characteristics and previous care; a monthly event tracker about recent treatments, healthcare visits, adverse events and productivity losses; and a monthly selection of validated patient-reported outcomes tools about MG symptoms, functioning and quality of life. To promote engagement, participants will receive in-app reminders to contribute data, acknowledgement for received data, and updates on study findings. The application will also support participants by providing clinician-validated information about $\mathrm{MG}$ and its management.

Discussion: Collecting data from people with MG via a smartphone application should enable a rich and granular dataset to be generated, to help researchers and clinicians understand the real-world impact of the condition and the unmet needs of patients.

Guillain Barrè syndrome chameleon: gadolinium magnetic resonance helps in early diagnosis.

A. Salvalaggio ${ }^{1,2}$, F. Castellani ${ }^{1}$, M. Anglani, ${ }^{3}$, M. Campagnolo ${ }^{1}$, R. Manara $^{3,4}$, C. Briani ${ }^{1}$.

${ }^{1}$ Neurology Clinic, Department of Neuroscience, University of Padova, Italy

${ }^{2}$ Padova Neuroscience Center, University of Padova, Italy

${ }^{3}$ Neuroradiology Unit, Azienda Ospedale Università di Padova, Padova, Italy

${ }^{3}$ Department of Neuroscience, University of Padova, Italy

Introduction: Chameleons or atypical presentations of Guillain Barré syndrome (GBS)-related disorders may be a challenge in acute clinical setting when ruling out alternative diagnosis is mandatory and when electrophysiology and cerebrospinal fluid (CSF) examination may still be negative. We report on a 38-year-old man with BGBS in whom a brainstem stroke was initially suspected.

Methods: The patient came to our attention for numbness and motor impairment at left-side of the face, dysgeusia and weakness at right lower limb. Neurological examination, brain and spine gadolinium MR, electrophysiological study and CSF examination were performed within three days from the onset of symptoms.

Results: Neurological examination revealed hypophonia, weakness at lower limbs, prominent on the right side (3/5 MRC in proximal and distal muscles on the right, $4 / 5$ on the left), distal paresthesias at feet and left peripheral facial nerve palsy. Achilles tendon reflexes were absent. Brain MR with gadolinium revealed left V and VII cranial nerves enhancement, and spine MR showed enhancement of cauda equina roots. CSF analysis and electrophysiological study were unremarkable the day after the onset.
The patient was diagnosed with GBS and treated with intravenous immunoglobulin $(2 \mathrm{~g} / \mathrm{kg})$ with prompt and complete recovery.

Discussion: The present case shows that in acute stage of atypical GBS, when electrophysiological study and CSF are still negative, MR may be crucial for confirming the clinical diagnosis. Notably, the trigeminal involvement has rarely been reported in GBS.

Testing a novel dasatinib formulation in $m d x$ mouse model: towards the repurposing of Src tyrosine kinase inhibitors in Duchenne muscular dystrophy

F. Sanarica ${ }^{1}$, P. Mantuano ${ }^{1}$, B. Boccanegra ${ }^{1}$, O. Cappellari ${ }^{1}$, E. Conte ${ }^{1}$, G. M. Camerino ${ }^{1}$, A. Cutrignelli ${ }^{2}$, N. Denora ${ }^{2}$, A. Mele ${ }^{1}$, M. De Bellis ${ }^{1}$, A. De Luca ${ }^{1}$

${ }^{1}$ Section of Pharmacology, Department of Pharmacy - Drug Sciences, University of Bari Aldo Moro, Bari, Italy

${ }^{2}$ Department of Pharmacy - Drug Sciences, University of Bari Aldo Moro, Bari, Italy

Overexpression and overactivation of the redox-sensitive protein SrcTyrosine Kinase (TK) in dystrophin-deficient muscles can contribute to $\beta$-dystroglycan ( $\beta$-DG) degradation and to reinforce damaging signaling, being therefore an interesting drug target. We have recently focused on preclinical studies in $m d x$ mouse for repurposing the Src-TK's competitive inhibitor dasatinib in Duchenne Muscular Dystrophy (DMD). A 4week subcutaneous treatment $(5 \mathrm{mg} / \mathrm{kg})$ with dasatinib lead to a muscle $\beta$ DG increment, although with limited efficacy on pathology-related indices [Sanarica et al., Pharmacol Res 2019]. We presently tested a new oral formulation (dasatinib-hydroxypropyl- $\beta$-cyclodextrin inclusion complex) to overcome possible pharmacokinetic issues and perform a longer treatment (10 and $20 \mathrm{mg} / \mathrm{kg}$ for 12 weeks) in drinking water. This newly formulated dasatinib reached plasma, liver and quadriceps in a dose-related fashion. In vivo, dasatinib contrasted the impaired exercise performance during an exhaustion treadmill test, with a recovery score (R.S.) toward wild type value of $28 \%(10 \mathrm{mg} / \mathrm{kg})$ and $17 \%$ $(20 \mathrm{mg} / \mathrm{kg})$, but did not exert any effect on $m d x$ mouse weakness, assessed by forelimb grip test. Ex vivo, dasatinib at $10 \mathrm{mg} / \mathrm{kg}$ ameliorated tetanic isometric force of both EDL muscle (R.S. 31\%) and diaphragm (R.S. $45 \%$ ), and partially recovered the elastic properties of this latter during eccentric contraction. Dasatinib, either 10 or $20 \mathrm{mg} / \mathrm{kg}$, decreased plasma levels of pathology-related biomarkers creatine kinase (R.S. $28 \%$ and $47 \%$ ) and lactate dehydrogenase (R.S. 36\% and 46\%). These preliminary data support the interest of this new oral formulation; however further experiments are necessary to better establish the therapeutic value of dasatinib repurposing for DMD treatment. [Supported by DPPNL_2015]

\section{Myastenia Gravis and Latent Tuberculosis Infection- A Case Report}

M. Sardaro, I. Plasmati, S. Aniello, M. Superbo, D. Liuzzi, S. Altomare, R. Leone, M. Giorelli, V. Lucivero

\section{U.O. Neurologia -P.O. Mons. Dimiccoli di Barletta- ASL BT}

Introduction: Myasthenia gravis (MG) is an autoimmune disease often treated with immunomodulatory and immunosuppressant agents which may increase the high risk of opportunistic infections including tuberculosis. Latent tuberculosis infection (LTBI) is characterised by the presence of immune responses to Mycobacterium tuberculosis infection without clinical evidence of active tuberculosis (TB). [1].

Case: We present the case of a 77 years old male with diplopia, eyelid ptosis (QMGTF: 4). Anti-AChR antibodies had increased (13.6 nmol/1 v.n<0.45). Chest CT: calcified micronodule localized to the right lobe and 
pleura. Interferon-gamma-release assays, Mantoux tuberculin skin: positive tests. The patient was diagnosed as having "Ocular Myasthenia Gravis, late-onset, thymoma-negative, anti-AChR antibodies-positive, LTBI" and he treated with pyridostigmine with remission of symptoms. Two months later worsening of symptoms (QMGTF:19). The patient began therapy with isoniazid, rifampicin and IVIg with progressive improvement of symptoms (QMGTF:4). No signs of active tuberculosis, seven months later so cortisonic therapy was initiated. The clinical status of the patient after one year of follow-up (ongoing) is unchanged.

Discussion: The lifetime risk of reactivation TB for a person with documented LTBI is estimated to be $5-15 \%$. The reactivation of TB can be averted by preventive treatment [2]. Our patient due to the risk of progression to TB was initially treated with IVIg before cortisonic therapy. Conclusion: Despite the significant prevalence of LTBI, TB and MG there are few data reported in the literature on clinical management and prognostic factors about these conditions with the need to refer to other autoimmune diseases. Further studies are mandatory to better explain of these disorders for a satisfying clinical management.

[1] Tuberculosis in myasthenia gravis. Ou SM, Liu CJ, Chang YS, Hu YW, Chao PW, Chen TJ, Tzeng CH, Wang SJ. Int J Tuberc Lung Dis . 2013 Jan;17(1):79-84.

[2] Management of latent Mycobacterium tuberculosis infection: WHO guidelines for

low tuberculosis burden countries. Getahun H. et al. Eur Respir J 2015; 46: $1563-1576$.

\section{Recurrent Variant of Miller Fisher Syndrome: is a ganglionopathy? - A Case Report}

M. Sardaro, I. Plasmati, C. Santoro, R. Calabrese, S. Aniello, S. Altomare, R. Leone, M. Giorelli, V. Lucivero

\section{U.O. Neurologia -P.O. Mons. Dimiccoli di Barletta- ASL BT, Italy}

Introduction: Miller-Fisher Syndrome (MFS) and Bickerstaff Brainstem Encephalitis (BBE) are rare variants of Guillan-Barrè Syndrome (GBS). They form a spectrum of post-infectious, autoimmune diseases sometimes characterized by overlapping symptoms and signs (GBSSpectrum-Disease "GBS-SD") [1].

Case: In 2015 a 16 years old girl developed headache, paresthesias, ataxic gait, diffuse areflexia, ophthalmoparesis, hypopallesthesia, lethargy. Brain MRI, cerebro-fluid-spinal examination, anti-ganglioside-complex and anti-AChR antibodies, nerve conduction studies, repetitive nerve stimulation, electromyography proved normal. The patient received therapy with IVIg and after 6 weeks she recovered.

In 2018 headache, ophthalmoplegia, areflexia, ataxia, bilateral facial muscle paralysis, hypophony, dysphagia, mild dyspnea, reappeared and progressively worsened. Cerebro-fluid-spinal examination revealed cytological-albumin dissociation. IgG anti-GQ1b and antiGT1a were positive. Sensory nerve action potentials (SNAP) showed significant decrease in amplitude on serial nerve conduction studies performed on the second and fourth day. The patient was diagnosed as having MFS-GBS-SD and treated with plasma exchange. After three weeks there was clinical remission.

Discussion: GBS-SD is a condition characterized by monophasic clinical course, relapses are rare [2]. In our opinion, this case is interesting because it combines several atypical aspects. Serial electrophysiological studies allowed to understand the underlying pathophysiology. Indeed, the reduction after four days in SNAP amplitude seems to contradict the pathogenetic hypothesis of exclusive neuronopathic involvement (ganglionopathy) as the cause of sensory signs and ataxia in MFS [3].

Conclusion: MFS and GBS-SD are complex syndromes. The characterization of the clinical and neurophysiological aspects could be useful to understand the pathogenetic mechanisms of these conditions and for the consequent therapeutic choices.
References:

[1] Update: the Miller Fisher variants of Guillain-Barré syndrome. Al Othman B, Raabe J, Kini A, Lee AG. Curr Opin Ophthalmol. 2019 Nov;30(6):462-466.

[2] Recurrent Miller Fisher; a new case reporter and a literature review. Barbato et al. Clin Ter 2017;168 (3):e 208-213.

[3] Electrophysiology in Fisher syndrome. Kuwabara et al. Clin Neurophysiol. 2017 Jan;128(1):215-21

Clinical and biological characterization of a large series of late-onset CMT2I patients carrying the MPZ P70S mutation

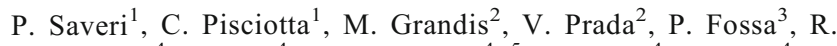
Mastrangelo $^{4}$, C. Ferri ${ }^{4}$, G. Shackleford ${ }^{4}{ }^{5}$, F. Veneri ${ }^{4}$, R. Baldi ${ }^{4}$, G. Lauria $^{1,6}$ R. Lombardi ${ }^{1}$, C. Ciano ${ }^{1}$, S. Magri ${ }^{1}$, F. Taroni ${ }^{1}$, L. Richard ${ }^{7}$. J.M. Vallat ${ }^{7}$, M. D’Antonio ${ }^{4}$, D. Pareyson ${ }^{1}$.

${ }^{1}$ Fondazione IRCCS Istituto Neurologico Carlo Besta, Milan, Italy;

${ }^{2}$ University of Genoa and IRCCS AOU San Martino-IST, Genoa, Italy;

${ }^{3}$ University of Genoa, Genoa, Italy;

${ }^{4}$ San Raffaele Scientific Institute, Milan, Italy;

${ }^{5}$ Hunter James Kelly Research Institute, Buffalo, NY USA;

${ }^{6}$ University of Milan, Milan, Italy;

${ }^{7}$ CHU de Limoges - Hôpital Dupuytren, Limoges Cedex, France.

The most common MPZ mutation causing late-onset CMT2J/I is T124M, all the other mutations are represented by few cases or single reports/ families. We have identified 42 CMT2I patients with the P70S mutation in Northern Italy (Laurà et al, JNNP 2007;78:1263-6). We are currently studying: their clinical and electrophysiological phenotype; the nodoparanodal regions in skin and nerve biopsies, as axo-glial interaction dysfunction has been hypothesized for CMT2I/J mutations (Brügger et al, PLoS Biol 2015;13:e1002258); the abnormalities of P70S cellular and mouse models.

We have enrolled 14 P70S-CMT2I patients (6 females; aged 33-78 years) with a mild-to-moderate phenotype in all but two paucisymptomatic patients (CMTES 0-13/28). Upper limb motor NCVs were in the CMT2 range $(42-60 \mathrm{~m} / \mathrm{s})$. We collected skin samples from 8 patients. In immunohistochemistry, preliminary results suggest paranodal abnormalities. At EM, we observed possibly atypical hemi-nodes in few axons of a sural nerve. In vitro studies demonstrated normal trafficking of the P0-P70S mutant. P70S/+ and P70S/P70S mice have been generated: none of them, examined at 12-18 months (due to the late disease onset in humans) showed any behavioural or neurophysiological abnormality. Further analyses are ongoing to study histological features at 18 and 24 months. This large cluster of CMT2I patients, that likely share a common founder, represents a valid model to study the phenotype of this late-onset $M P Z$ related CMT (which is milder than CMT2J) and the pathomechanisms of axo-glial interactions.

Supported by grants AFM-Telethon \#20667 and Telethon-Italy GPP19099.

Spinal cord volumetry as a biomarker for monitoring drug treatment in spinal muscular atrophy patients: a pilot MRI study

G. Savini $^{1,2}$, C. Asteggiano ${ }^{2}$, L. M. Farina ${ }^{2}$, C. A. M. Gandini WheelerKingshott $^{1,3,4}$, S. Bastianello ${ }^{2,3}$, A. Berardinelli ${ }^{5}$, A. Pichiecchio ${ }^{2,3}$

${ }^{I}$ Brain MRI 3 T Research Center, IRCCS Mondino Foundation, Pavia, Italy

${ }^{2}$ Neuroradiology Unit, IRCCS Mondino Foundation, Pavia, Italy

${ }^{3}$ Department of Brain and Behavioral Sciences, University of Pavia, Pavia, Italy 
${ }^{4}$ Queen Square Multiple Sclerosis Centre, Department of Neuroinflammation, UCL Queen Square Institute of Neurology, University College London, London, UK

${ }^{5}$ Child Neurology and Psychiatry Unit, IRCCS Mondino Foundation, Pavia, Italy

Spinal muscular atrophy (SMA) is a rare genetic neuromuscular disorder that results in the loss of motor neurons located in the ventral horns $(\mathrm{VH})$ of the spinal cord (SC). Nusinersen is a medication that is administered using an intrathecal injection and that was shown to slow disease progression and to improve motor function. Cervical SC (CSC) magnetic resonance imaging (MRI) has been used to assess cord atrophy in patients affected by SMA, but it has never been used to monitor drug treatment. 3 female patients affected by SMA and under treatment with nusinersen and 7 female age-matched healthy controls (HC) underwent a first CSC 3T-MRI session (TP1). SMA patients underwent a second MRI session after 4 months (TP2). We computed the cross-sectional area (CSA) of the whole SC, grey matter (GM) and VH. We compared values of CSA between HC and SMA groups at TP1 and also between time points for patients.

The comparison between $\mathrm{HC}$ and patients at TP1 showed that all computed CSA values of all patients fell in the lower range of the $\mathrm{HC}$ group. The comparison between different time points of SMA patients showed an overall mild increase of CSA values at TP2.

To the authors knowledge this is the first attempt to use MRI-derived CSC volumes as biomarkers of drug treatment in SMA. Although the sample size does not allow us to draw certain conclusions yet, our results seem to suggest that treatment with nusinersen has an effect on SC and GM volume.

Hereditary neuropathy with liability to pressure palsy: two pediatric symptomatic cases with acute monolateral proof drop

G. Scarpini ${ }^{1}$, M. Giannotta ${ }^{1}$, F. Pastorelli ${ }^{2}$, A. Pini ${ }^{1}$

1. UO Neuropsichiatria Infantile Neuromuscular Pediatric Centre and ${ }^{2}$ UO Neurologia, IRCCS, Istituto delle Scienze Neurologiche di Bologna Ospedale Bellaria, Italy

Hereditary Neuropathy with Liability to Pressure Palsy (HNPP) is a rare disorder with a genetic susceptibility to paralysis after minor trauma and/or compression. This disorder has an autosomic dominant inheritance and it is characterized by recurrent remitting acute painless sensory-motor mono-neuropathies frequently involving peroneal or ulnar nerve. Recovery may not be total and a motor deficit may persist in time after the acute phase. Sometimes the symptomatology is misleading and a correct diagnosis is probably underestimated since a genetic disease is generally not investigated. In most cases, this condition is caused by a deletion on the PMP 22 gene located on 17 p11.2 and first symptoms appear in the second or third decade of life. Few pediatric cases are reported and the treatment is not clearly established. We report here two unrelated pediatric cases with acute onset arrived in the emergency room. The first was a male 7 years old with acute onset of right foot drop, who had reported a fracture of the 5 metatarsal about a month earlier; the second was a male 10 years old who noticed the sudden onset of right foot drop and personal history revealed a sprained right ankle emerged about 2 days before. Neuropaediatric counselling has raised the suspicion of HNPP, the electroneurography demonstrated transient sensory and motor peroneal nerve involvement and genetic analisys revealed a heterozygous deletion of PMP22 gene. As a consequence the clinical and genetic investigations were extended to other family members and advice to avoid positions potentially causing further compression were given.
Report of an unusual course of CANDA (chronic ataxic neuropathy with disialosyl antibodies) E.Schirinzi ${ }^{1}$, E. Merico ${ }^{1}$, C. Simoncini ${ }^{1}$, A. Govoni ${ }^{1}$, A. Bacci ${ }^{1}$, R.
Calabrese $^{1}$, G. Siciliano

${ }^{1}$ Department of Clinical and Experimental Medicine, Neurological Clinic, University of Pisa, Italy

CANDA is a rare progressive and disabling peripheral neuropathy with characteristic clinical and diagnostic findings

We report the case of a 77-year-old woman which developed a gait disorder within last 10 years without initial suggestive findings of CANDA. In her anamnesis we noticed a parossistic AF and a 30 aged son died of sudden death. The neurological examination confirmed only a moderate ataxia and spastic paraparesis during the deambulation. Total spine MRI did not detect any cord lesion. Cerebral MRI showed a slight supratentorial cortical and subcortical atrophy and a bilateral atrophy of the cerebellar cortex. Electromiography and motor evoked potentials were negative, electroneurography was suggestive of a slight right TCS. No systemic diseases were found. Searching for genetic spino-cerebellar ataxia and spastic paraparesis was negative. A diagnosis of cerebellar vermian ataxia was supposed.

During the last months she reported paresthesia distally at lower limbs. At neurological examination a left foot drop, tendon hyporeflexia and distal sensory loss were found. Electroneurography showed a diffuse slight axonal-demyelinating pattern.

A slight ventricular hypertrophy at the transthoracic echocardiography and a IgM monoclonal gammopathy were detected. Genetic searching for aTTR amyloidosis was negative but a high titer of IgM-GD1b-GD2-GD3 antiganglioside antibodies was found. A diagnosis of CANDA was postulated and IVIG therapy was started with a clinical improvement.

Few reports are available about CANDA. Tipically it is described associated to IgM-GD1b ganglioside antibodies and not responsive to IVIG. The clinical course and the antibodies pattern of our case expands the previously clinical spectrum described for these neuropathy.

Familial mitochondrial myopathy and renal failure due to biallelic MGME1 mutations

M. Sciacco ${ }^{1}$, M. Ripolone ${ }^{1}$, L. Napoli ${ }^{1}$, D. Piga ${ }^{2}$, D. Velardo ${ }^{1}$, P, Ciscato ${ }^{1}$, M. Moggio ${ }^{1}$, G.P. Comi ${ }^{1,2}$, D. Ronchi ${ }^{2}$

${ }^{1}$ Neuromuscular and Rare Disease Unit, Department of Neuroscience, IRCCS Foundation Ca' Granda Ospedale Maggiore Policlinico, University of Milan, Milan, Italy.

${ }^{2}$ Department of Pathophysiology and Transplantation (DEPT), Dino Ferrari Centre, University of Milan, IRCCS Foundation Ca' Granda Ospedale Maggiore Policlinico, Milan, Italy.

We describe a 45 y.o. woman whose clinical history dates back to the age of 19 years when she developed easy fatigability associated with slight iperckemia. A needle muscle biopsy was consistent with a diagnosis of mitochondrial disorder. She did not report other symptoms until few years ago when she noticed occasional diplopia followed, two years ago, by bilateral eyelid ptosis. At the age of 34 years, while she was pregnant, she was diagnosed hypertension and renal failure associated with bilateral kidney hypotrophy. She has been undergoing dialysis for one year. Brain MRI is normal except for the presence of focal, unspecific T2Flair hyperintensities.

The patient's only sibling, a 44 y.o. sister, has moderate hyperckemia, but no renal failure, however, she manifested bilateral eyelid ptosis as a teenager and she underwent corrective surgery three years ago.

Parents are non-consanguineous though coming from the same geographical area. 
Patient's neurological examination shows marked, bilateral eyelid ptosis with limitation of eye movements in all directions. She recently underwent a second muscle biopsy which shows several COX-negative fibers, RRFs and marked ultrastructural mitochondrial abnormalities. Multiple mtDNA deletions were documented in proband's muscle by long-range-PCR. Whole exome sequencing identified biallelic MGME1 defects in the proband. MGME1 mutations were previously detected only in three independent families presenting childhood-/adult-onset progressive external ophthalmoplegia, proximal weakness, profound emaciation and respiratory failure and in a child manifesting early onset cerebellar ataxia. Our cases expand the clinical and molecular findings and highlight the progressive muscle mitochondrial dysfunction associated with MGME1 mutations.

\section{Whole exome and genome sequencing for the genetic diagnosis of dystrophinopathies}

R. Selvatici ${ }^{1}$, R. Rossi ${ }^{1}$, F. Mingyan ${ }^{2}$, M.S. Falzarano ${ }^{1}$, P. Rimessi ${ }^{1}$, M. Neri $^{1}$, F. Gualandi ${ }^{1}$, Sanja Delin ${ }^{3}$, S. Bensemmane ${ }^{4}$, Andriy Shatillo ${ }^{5}$, L. Bello $^{6}$, E. Pegoraro ${ }^{6}$, A. Ferlini ${ }^{1}$

${ }^{1}$ Unit of Medical Genetics, Department of Medical Sciences, UniversityHospital of Ferrara, Italy.

${ }^{2}$ BGI-Shenzhen, Shenzhen, 518083, China.

${ }^{3}$ Department of Pediatric, General Hospital Zadar, Zadar, Croatia.

${ }_{5}^{4}$ Neurologie,E.H.S. Ali Ait-Idir, Alger

${ }^{5}$ Institute of Neurology, Psychiatry and Narcology, Kharkiv, Ukraine.

${ }^{6}$ Department of Neuroscience, Unit of Neurology, University of Padua, Italy

Dystrophinopathies are allelic diseases caused by dystrophin (DMD) mutations leading to DMD and BMD phenotypes. Quantitative (MLPA and/ or CGH array) and qualitative (NGS, Sanger-sequencing) methods are routinely used for genetic diagnosis, accordingly to existing guidelines (EMQN). Nevertheless, about $1 \%$ of atypical, complex rearrangements or intronic changes remain unsolved.

We run WES in 28 DMD patients. We demonstrate that WES is a sensitive method to pick up either deletions and small mutations in DMD males, but not duplications. Its adoption allows a detection rate close to $90 \%$ of DMD gene mutations therefore it might be considered a "first step" testing for DMD, especially in considering the emerging ethnicspecific mutation landscapes. WES can identify a multitude of SNPs , including those known as gene modifiers, recently associated with DMD phenotypic variability providing further useful information about the genotype/haplotype of DMD boys.

We also run whole genome sequencing (WGS) in two DMD cases in which conventional methods did not identify any DMD mutation. In one patient, WGS revealed the presence of a $15 \mathrm{Mb}$ complex rearrangement from chrXp21.2 to Xp22.2, involving intron 54 of the DMD gene and GRPR gene (located in Xp2.2). In the second patient we found a $9.4 \mathrm{Mb}$ inversion from chrXp21.1 to Xp22.12, involving intron 2 of the DMD gene and an upstream intergenic region. These high throughput techniques will tremendously impact on DMD genetic diagnosis, but we should also be reasoning about time-to-report needs when personalized therapies are available, in order to apply the appropriate diagnostic pipeline.

Real-World Treatment Patterns and Outcomes in Patients With Spinal Muscular Atrophy Collected From the RESTORE Registry

L. Servais ${ }^{1}$, J.W. Day ${ }^{2}$, D.C. De Vivo ${ }^{3}$, J. Kirschner ${ }^{4}$, E. Mercuri ${ }^{5}$, F. Muntoni $^{6}$, P.B. Shieh ${ }^{7}$, E. Tizzano ${ }^{8}$, I. Desguerre ${ }^{9}$, S. Quijano-Roy ${ }^{10}$, K. Saito $^{11}$, M. Droege ${ }^{12}$, O. Dabbous ${ }^{12}$, R. Cerbini ${ }^{12}$, F. Baldinetti ${ }^{12}$, A. Shah $^{12}$, F. Khan ${ }^{12}$, F.A. Anderson ${ }^{13}$, R.S. Finkel ${ }^{14}$
${ }^{1}$ MDUK Oxford Neuromuscular Centre, University of Oxford, Oxford, United Kingdom

${ }^{2}$ Department of Neurology, Stanford University Medical Center, Stanford, CA, United States

${ }^{3}$ Departments of Neurology and Pediatrics, Columbia University Irving Medical Center, New York, NY, United States

${ }^{4}$ Clinic for Neuropediatrics and Muscle Disease, University Medical Center Freiburg, Freiburg, Germany

${ }^{5}$ Department of Paediatric Neurology and Nemo Clinical Centre, Catholic University, Rome, Italy

${ }^{6}$ Department of Developmental Neuroscience, University College London, London, United Kingdom

${ }^{7}$ Department of Neurology, David Geffen School of Medicine at UCLA, Los Angeles, CA, United States

${ }^{8}$ Department of Clinical and Molecular Genetics, Hospital Valle Hebron, Barcelona, Spain

${ }^{9}$ Hopital Necker Enfants Malades, APHP, Paris, France

${ }^{(10)}$ Garches Neuromuscular Reference Center (GNMH), APHP Raymond Poincare University Hospital (UVSQ Paris Saclay), Garches, France

${ }^{11}$ Institute of Medical Genetics, Tokyo Women's Medical University, Tokyo, Japan

${ }^{12}$ AveXis, Inc., Bannockburn, IL, United States

${ }^{13}$ Center for Outcomes Research, Department of Surgery, University of Massachusetts Medical School, Worcester, MA, United States

${ }^{14}$ Division of Neurology, Department of Pediatrics, Nemours Children's Hospital, Orlando, FL, United States

Introduction: Until recently, supportive care was the sole treatment option for patients with spinal muscular atrophy (SMA). Disease-modifying treatments (eg, gene therapy, antisense oligonucleotides) are now available and have dramatically improved prognosis; however, real-world outcome data remain limited. Thus, there is a need for long-term efficacy and safety information from patients receiving both existing and new therapies to describe the impact of intervention on patients, caregivers, and the healthcare system. RESTORE is a prospective, multicenter, treatmentagnostic registry of patients with SMA specifically designed to integrate and expand available real-world data, thus overcoming the recognized limitations of existing single-product registries.

Methods: The RESTORE database incorporates data from patients enrolled in partnering registries and the onasemnogene abeparvovec (formerly AVXS-101) managed-access program. Follow-up duration is 15 years from enrollment or until death.

Results: As of 5 March 2020, the RESTORE database comprises information from 88 patients and 35 active sites in the United States. This cohort permits descriptive analyses of patients with a range of baseline characteristics at time of dosing, including individuals who have switched therapies, and who received treatment under managed/expanded-access programs at a variety of treatment centers. RESTORE is rapidly expanding globally, with 51 sites currently in start-up.

Conclusion: RESTORE, a comprehensive registry of patients with SMA, provides extended real-world assessments of patient outcomes and SMA interventions. RESTORE is rapidly expanding globally, allowing for future descriptive analysis of regional treatment patterns and outcomes.

\section{Hereditary Transthyretin Amyloidosis (hATTR) Polyneuropathy:} experience from a single center.

D. Severi, S. Tozza, E. Spina, M. Bellofatto, M. Nolano, L. Santoro, F. Manganelli.

Department of Neuroscience, Reproductive Sciences and Odontostomatology, University of Naples "Federico II", Naples, Italy.

We reported demographic and clinical features of a cohort of hATTR patients, referred to University Federico II of Naples, consisting of 15 
patients from 14 families carrying TTR mutations (Val30Met: 8; Phe64Leu: 5). Eleven families (Val30Met: 7) were from Campania, 1 from Lazio, 1 from Calabria, and 1 from Puglia.

Male to female ratio was $14: 1$, mean age of onset $65.5 \pm 5.9$ years (Val30Met 65.2 \pm 7.1 vs Phe64Leu 66.1 \pm 4 ), mean current age 71.9 \pm 5.8 years (Val30Met $72.3 \pm 7$ vs Phe64Leu $71 \pm 4.5$ ), mean disease duration $6.4 \pm 3.6$ years (Val30Met $7.1 \pm 3.9$ vs Phe64Leu 5.4 \pm 2.7 ), mean follow-up period $25 \pm 22.2$ months (Val30Met $23.8 \pm 27.1$ vs Phe64Leu 25.4 \pm 17.1 ) and mean diagnostic delay $3.2 \pm 1.7$ years (Val30Met $3.5 \pm 1.6$ vs Phe64Leu $2.7 \pm 0.9$ ). Two patients (Phe64Leu) died after $8 \pm 4.2$ years ( 1 from cardiac failure, 1 from pneumonia). Thirteen patients had neuropathic phenotype at onset and nine (Val30Met: 6) of them developed cardiac involvement. Nine patients complained autonomic symptoms (Val30Met: 5). Five patients are in treatment with Tafamidis, 4 with Patisiran, 3 with Inotersen and 1 with Diflunisal.

For the 9 patients that were under Tafamidis, we evaluated changes of laboratory and clinical data (NIS, CMTNS, Kumamoto and Norfolk QOL-DN) over a period of 18 months. ANOVA for repeated measures showed that only Kumamoto $(p=0.008)$ and NIS $(p=0.0008)$ scores worsened over time.

Our hATTR cohort was characterised by similar prevalence of Val30Met and Phe64Leu, male predominance, late onset, and predominant neuropathic phenotype.

Our data, in keeping with literature, showed that mean diagnostic delay was 3 years, as well disease progressed over time despite treatment.

\section{Proteomic profiling of cerebrospinal fluid of nusinersen-treated SMA1 patients}

M. Sframeli ${ }^{1}$, L. Bianchi ${ }^{2}$, G.L. Vita ${ }^{1}$, R. Oteri ${ }^{3}$, F. Polito ${ }^{3}$, L. Vantaggiato $^{2}$, C. Landi ${ }^{2}$, E. Gitto ${ }^{4}$, S. Messina ${ }^{3}$, L. Bini ${ }^{2}$, M. Aguennouz ${ }^{3}$, G. Vita ${ }^{1,3}$

${ }^{I}$ Nemo Sud Clinical Centre, Messina, Italy

${ }^{2}$ Functional Proteomics Laboratory, Life Sciences Department, University of Siena, Siena, Italy

${ }^{3}$ Unit of Neurology and Neuromuscular Diseases, Department of Clinical and Experimental Medicine, University of Messina, Messina, Italy

${ }^{4}$ Neonatal and Paediatric Intensive Care Unit, Department of Human Pathology in Adult and Developmental Age, University of Messina, Messina, Italy

Spinal muscular atrophy (SMA) type 1 is the infantile form of SMA characterized by severe and progressive muscle weakness. The affected children did not achieve the ability of independent sitting, however the recent advent of new therapies, such as the antisense oligonucleotide nusinersen, has significantly improved the natural course of the disease. In order to monitor the course of the disease according to the therapy, it is useful to identify one or more biomarkers. 14 patients (age range: 2-156 months) with SMA type 1 were evaluated at baseline and after six months of treatment with nusinersen. Patients were assessed using the CHOP-INTEND functional scale. 2-D gel electrophoresis and mass spectrometry for protein profiling of cerebrospinal fluid (CSF) were performed. Control CSF samples were obtained from age-matched subjects investigated for dysimmune neurological diseases and then resulted not affected. The treatment led to a significant variation in CSF protein pattern and, in particular, it resulted in a generalized reversion trend of the SMA1 protein profile comparing with the baseline, specifically proteins involved in: neurodegeneration, inflammation, and potential redox status. Further analyzes are in progress to explore mechanisms explaining these findings and to clarify which differential molecular networks are modulated by nusinersen administration.
Mitochondrial abnormalities with mtDNA single deletion in association with a LMNA gene frameshift variant: a case report.

C. Simoncini ${ }^{1}$, V. Montano ${ }^{1}$, G. Ricci1, G. $\mathrm{Ali}^{2}$, A. Logerfo ${ }^{1}, \mathrm{~F}$. Baldinotti $^{3}$, M.A.Caligo ${ }^{3}$, G. Lattanzi ${ }^{4}$, G. Cenacchi ${ }^{5}$, A. Barison ${ }^{6}$, M. Mancuso $^{1}$, G. Siciliano ${ }^{1}$

${ }^{1}$ Dept. of Clinical and Experimental Medicine, University of Pisa.

${ }^{2}$ Dipartimento di Patologia Chirurgica, Medica, Molecolare e Dell'Area Critica, University of Pisa, Pisa, I-56126 Pisa, Italy.

${ }^{3}$ SOD Genetica Molecolare, Azienda Ospedaliero-Universitaria Pisana, Pisa, Italy.

${ }^{4}$ CNR Institute of Molecular Genetics "Luigi-Luca Cavalli-Sforza" Unit of Bologna, Bologna, Italy; IRCCS Istituto Ortopedico Rizzoli, Bologna, Italy

${ }^{5}$ Department of Biomedical and Neuromotor Science, ALMA MATER, University of Bologna 6 U.O.C. Fondazione G. Monasterio CNRRegione Toscana, Pisa, Italy.

A 63 year old man arrived to our attention for an one-year history of muscle cramps, exercise intolerance, progressive proximal weakness at the lower limbs and mild hyperCKemia (400 UI/L). Family history was negative for neuromuscular disease. In anamnesis he referred only arterial hypertension.

The neurological examination showed asymmetric winged scapula, mild hypotrophy of right brachial biceps and femoral quadriceps with bilateral motor deficit in leg extension (MRC 4/5). The electromyography revealed a myopathic pattern with spontaneous activity (fibrillation potentials) in tibialis anterior muscles. Myositis specific antibodies were negative. Forearm ischemic test showed basal hyperlactacidemia. The deltoid muscle biopsy revelead mitochondrial abnomalities (in particular scattered ragged-red fibers and several COX negative fibres). Muscle MRI showed a diffuse involvement of thigh and leg muscles (in particular fatty infiltration and mild edema). Mitochondrial DNA long-range PCR analysis disclosed a single deletion in the mtDNA. Moreoever, the NGS analysis of limb girdle muscle dystrophies/myopathies genes panel identified a previously unreported heterozygous variant in exon 11 of the LMNA gene (c.1861dupA, p.Thr621Asnfs*83). The variant is predicted to disrupt protein reading frame and to determine the loss of the original stop codon with formation of a longer protein. In silico predictions supported his pathogenicity. A detailed cardiological assessment showed first-degree atrioventricular block and a small late enhancement area in the interventricular septum at cardiac magnetic resonance. Implication deriving from hypothetical coexistence of the two different pathological conditions will be discussed and further details will be added on in site discussion

\section{The neurophysiological lesson from the italian CIDP database}

E. Spina ${ }^{1}$, P. Emiliano Doneddu, ${ }^{2}$ G. Liberatore,${ }^{2}$ D. Cocito, ${ }^{3}$ R. Fazio, ${ }^{4}$ C. Briani,${ }^{5}$ M. Filosto, ${ }^{6}$ L. Benedetti, ${ }^{7},{ }^{8}$ G. Antonini, ${ }^{9}$ G. Cosentino, ${ }^{10} \mathrm{~S}$. Jann, ${ }^{11}$ A. Mazzeo, ${ }^{12}$ A. Cortese $,{ }^{13},{ }^{14}$ G. Alessandra M. ${ }^{15}$ A. Maurizio Clerici, ${ }^{16}$ G. Siciliano, ${ }^{17}$ M. Carpo, ${ }^{18}$ M. Luigetti, ${ }^{19}$ G. Lauria, ${ }^{20,}{ }^{21}$ T. Rosso, ${ }^{22}$ G. Cavaletti, ${ }^{23}$ E. Peci, ${ }^{3}$ S. Tronci, ${ }^{4}$ M. Ruiz, ${ }^{5}$ S. Cotti Piccinelli, ${ }^{6}$ A. Schenone, ${ }^{7}$ L. Leonardi, ${ }^{9}$ L. Gentile, ${ }^{12}$ L. Piccolo, ${ }^{13}$ G. Mataluni, ${ }^{15}$ L. Santoro, ${ }^{1}$ E. Nobile-Orazio, ${ }^{2,}{ }^{24}$ and F. Manganelli, ${ }^{1}$ on the behalf of the Italian CIDP Database Study Group.

1 Department of Neuroscience, Reproductive Sciences and Odontostomatology, University of Naples 'Federico II', Naples, Italy

${ }^{2}$ Neuromuscular and Neuroimmunology Service, Humanitas Clinical and Research Institute, Rozzano, Milan, Italy

${ }^{3}$ Presidio Sanitario Major, Istituti Clinici Scientifici Mangeri, Turin, Italy 
${ }^{4}$ Division of Neuroscience, Department of Neurology, Institute of Experimental Neurology (INSPE), San Raffaele Scientific Institute, Milan, Italy

${ }^{5}$ Neurology Unit, Department of Neuroscience, University of Padova, Padova, Italy

${ }^{6}$ Center for Neuromuscular Diseases and Neuropathies, Unit of Neurology, ASST 'Spedali Civili', University of Brescia, Brescia, Italy

${ }^{7}$ Department of Neuroscience, Rehabilitation, Ophthalmology, Genetics, Maternal and Child Health, University of Genoa and IRCCS San Martino, Genoa, Italy

${ }^{8}$ IRCCS AOU San Martino-IST, Genoa, Italy

${ }^{9}$ Unit of Neuromuscular Diseases, Department of Neurology Mental Health and Sensory Organs (NESMOS), Faculty of Medicine and Psychology, 'Sapienza' University of Rome, Sant'Andrea Hospital, Rome, Italy

${ }^{10}$ Department of Brain and behavioral Sciences, University of Pavia, Pavia, Italy. IRCCS Mondino Foundation, Pavia, Italy.

${ }^{11}$ Department of Neuroscience, Niguarda Ca' Granda Hospital, Milan, Italy

12 Department of Clinical and Experimental Medicine, Unit of Neurology, University of Messina, Messina, Italy

${ }^{13}$ IRCCS Foundation C. Mondino National Neurological Institute, Pavia, Italy

${ }^{14}$ Molecular Neurosciences, University College London, London, UK

${ }^{15}$ Dysimmune Neuropathies Unit, Department of Systems Medicine, Tor Vergata University of Rome, Rome, Italy

${ }^{16}$ Neurology Unit, Circolo \& Macchi Foundation Hospital, Insubria University, DBSV, Varese, Italy

${ }^{17}$ Neurology Unit, Department of Clinical and Experimental Medicine, University of Pisa, Pisa, Italy

${ }^{18}$ Neurology Unit, ASST Bergamo Ovest-Ospedale Treviglio, Treviglio, Italy

19 Fondazione Policlinico Universitario A. Gemelli IRCCS, UOC Neurologia, Università Cattolica del Sacro Cuore, Rome, Italy.

${ }^{20}$ Unit of Neuroalgology, IRCCS Foundation 'Carlo Besta' Neurological Institute, Milan, Italy

${ }^{21}$ Department of Biomedical and Clinical Sciences 'Luigi Sacco', University of Milan, Milan, Italy

${ }^{22}$ ULSS2 Marca Trevigiana, UOC Neurologia-Castelfranco Veneto, Treviso, Italy

${ }^{23}$ School of Medicine and Surgery and Experimental Neurology Unit, University of Milano-Bicocca, Monza, Italy.

${ }^{24}$ Department of Medical Biotechnology and Translational Medicine, Milan University, Milan, Italy

Objective: Electrophysiological diagnosis of CIDP may be challenging. Thus, with the aim of providing some practical advice in electrophysiological approach to a patient with suspected CIDP we evaluated electrophysiological data from the Italian CIDP Database.

Methods: We analyzed 419/499 patients fulfilling and 80/499 not fulfilling the EFNS/PNS electrophysiological CIDP criteria.

In fulfilling patients we calculated the rate of each demyelinating feature, the rate of demyelinating features per nerve and the diagnostic rate for upper and lower limb nerves, and, using a ROC curve analysis, the diagnostic accuracy of each nerve and demyelinating feature, in typical and in atypical CIDP subtypes. Moreover, we compared the electrophysiological data of fulfilling patients with those of not-fulfilling patients and by a logistic regression analysis we estimated the diagnostic odds ratio (OR). Results: In fulfilling patients the ulnar nerve had the highest rate of demyelinating features as well as the highest diagnostic accuracy in CIDP population as a whole and in each CIDP category except for DADS. Indeed, peroneal nerves followed by tibial nerves were the most informative nerves in DADS patients.

In not-fulfilling patients a lower number of nerves and temporal dispersion (TD) measurements had been performed compared to fulfilling patients. The univariate logistic regression analysis showed an OR of 1.41 and 1.28 for any tested motor nerve and TD measurement, respectively.

Conclusions: Electrophysiological approach to a patient suspected of CIDP should take into account the clinical phenotype. An adequate extension of the examination and an accurate interpretation of neurophysiological results are crucial not to miss the diagnosis of CIDP.

\section{A new Italian family with PMP2-related Charcot-Marie-Tooth Disease type 1G}

S. Spolverato ${ }^{1}$, F. Taioli ${ }^{2}$, G. Zanette ${ }^{3}$, S. Romito ${ }^{4}$, M. Ferrarini ${ }^{2}$, L. Roncari $^{5}$, G. Cantalupo ${ }^{5}$, G.M. Fabrizi ${ }^{2}$

${ }^{1}$ Child Neuropsychiatry Unit, University-Hospital of Verona, Verona, Italy

${ }^{2}$ Neurology Unit (B), University-Hospital of Verona, Verona, Italy

${ }^{3}$ Neurology Unit, Pederzoli Hospital, Peschiera del Garda, Italy

${ }^{4}$ Neurology Unit (A), University-Hospital of Verona, Verona, Italy

${ }^{5}$ Rehabilitation Unit, Pederzoli Hospital, Peschiera del Garda, Italy

The demyelinating autosomal dominant Charcot-Marie-Tooth (CMT1) disease has four major loci identified including the most common duplication of PMP22 in $17 \mathrm{p} 11.2$ (CMT1A). The peripheral myelin protein 2 gene (PMP2), encoding a protein involved in the transport of fatty acids and metabolism of myelin lipids, was added recently to CMT1 genes, linked to the presumably rare CMT1G.

A 11-year-old male proband was affected by an autosomal dominant motor-sensory polyneuropathy presenting with a peroneal atrophy syndrome (stepping gait and impairment of manual dexterity) associated with markedly and uniformly reduced nerve conduction velocities (NCV) in the upper limbs (motor NCV $14 \mathrm{~m} / \mathrm{s}$ ). The asymptomatic proband's father had a normal neurological examination, except pes cavus and generalized areflexia, with uniformly reduced NCV (motor $\mathrm{NCV}$ in the upper limbs $28 \mathrm{~m} / \mathrm{s}$ ). Nerve high-resolution ultrasound (HRUS) performed in the proband and his father did not reveal significant increases of the cross-sectional area, diverging from the HRUS pattern observed in the common CMT1A. After ruling out the common $17 \mathrm{p} 11.2$ duplication, targeted next generation sequencing (NGS) revealed that son and father shared a heterozygous c.146 148delTTA of $P M P 2$ which is predicted to cause a in-frame p.Ile50del deletion. The same mutation had been previously associated to CMT1G in a singleton, apparently unrelated, Italian family (Geroldi A et al., JPNS 2018).

The report confirms the causative role of $P M P 2$. Similarly to CMT1A, CMT1G may present a striking intrafamilial clinical variability that contrasts with a complete penetrance of NCV slowing. However, divergent HRUS findings indicate that CMT1A and CMT1G recognize different pathological bases.

Onasemnogene Abeparvovec Gene Therapy in Presymptomatic Spinal Muscular Atrophy: SPR1NT Study Update

K.A. Strauss ${ }^{1}$, M.A. Farrar ${ }^{2}, 3$, K.J. Swoboda ${ }^{4}$, K. Saito ${ }^{5}$, C.A. Chiriboga ${ }^{6}$, R.S. Finkel ${ }^{7}$, S.T. Iannaccone ${ }^{8}$, J.M. Krueger ${ }^{9}$, J.M. Kwon ${ }^{10}$, H.J. McMillan $^{11}$, L. Servais ${ }^{12}$, J.R. Mendel1 ${ }^{13-15}$, J. Parsons ${ }^{16}$, M. Scoto ${ }^{17}$, P.B. Shieh $^{18}$, C. Zaidman ${ }^{19}$, F. Baldinetti ${ }^{20}$, M. Schultz ${ }^{20}$, S.P. Reyna ${ }^{20}$, F.G. Ogrinc $^{20}$, S. Kavanagh ${ }^{20}$, D. Chand ${ }^{20}$, D.E. Feltner ${ }^{20}$, S. TauscherWisniewski $^{20}$, B.E. McGill ${ }^{20}$, D.M. Sproule ${ }^{20}$, F. Muntoni ${ }^{17,21}$

${ }^{1}$ Clinic for Special Children, Strasburg, PA, United States

${ }^{2}$ Department of Neurology, Sydney Children's Hospital Network, Randwick, NSW, Australia

${ }^{3}$ UNSW Sydney, Sydney, NSW, Australia 
${ }^{4}$ Department of Neurology, Massachusetts General Hospital, Boston, MA, United States

${ }^{5}$ Institute of Medical Genetics, Tokyo Women's Medical University, Tokyo, Japan

${ }^{6}$ Division of Pediatric Neurology, Columbia University Medical Center, New York, NY, United States

${ }^{7}$ Division of Neurology, Department of Pediatrics, Nemours Children's Hospital, Orlando, FL, United States

${ }^{8}$ Department of Pediatrics, University of Texas Southwestern Medical Center, Dallas, TX, United States

${ }^{9}$ Department of Neurology, Helen DeVos Children's Hospital, Grand Rapids, MI, United States

${ }^{10}$ Department of Neurology, University of Wisconsin School of Medicine and Public Health, Madison, WI, United States

${ }^{11}$ Department of Pediatrics, Children's Hospital of Eastern Ontario, Ottawa, ON, Canada

${ }^{12}$ MDUK Oxford Neuromuscular Center, Oxford, United Kingdom

${ }^{13}$ Center for Gene Therapy, Nationwide Children's Hospital, Columbus, $\mathrm{OH}$, United States

${ }^{14}$ Department of Pediatrics, Ohio State University, Columbus, OH, United State

${ }^{15}$ Department of Neurology, Ohio State University, Columbus, OH, United States

${ }^{16}$ Department of Pediatrics, University of Colorado School of Medicine, Aurora, CO, United States

${ }^{17}$ The Dubowitz. Neuromuscular Centre, University College London, Great Ormond Street Institute of Child Health \& Great Ormond Street Hospital, London, United Kingdom

${ }^{18}$ Department of Neurology, David Geffen School of Medicine at UCLA, Los Angeles, CA, United States

${ }^{19}$ Department of Neurology, Division of Pediatric Neurology, Washington University School of Medicine, St. Louis, MO, United States

${ }^{20}$ AveXis, Inc., Bannockburn, IL, United States

${ }^{21}$ National Institute of Health Research, Great Ormond Street Hospital Biomedical Research Centre, London, United Kingdom

Introduction: Spinal muscular atrophy (SMA) causes loss of motor/ respiratory function due to biallelic $S M N 1$ deletion/mutation. Copies of a similar gene (SMN2) modify disease severity. Here, we evaluate the safety/efficacy of onasemnogene abeparvovec (formerly AVXS-101) in presymptomatic SMA patients.

Methods: SPR1NT (NCT03505099) is an ongoing multicenter, open-label, phase 3 study. Asymptomatic patients $(2-3 \times S M N 2, \leq 6$ weeks) receive a one-time IV infusion and are assessed through 18 / $24(2 \times / 3 \times S M N 2)$ months. Primary outcomes: sitting $\geq 30$ seconds $(2 \times S M N 2) /$ standing unassisted $\geq 3$ seconds $(3 \mathrm{x} S M N 2)$. Exploratory outcome: motor function improvement (CHOP INTEND). Safety outcomes: incidence of adverse events (AEs)/serious AEs.

Results: As of 31 Dec 2019, 30 infants were dosed (2xSMN2/3xSMN2/ $4 \mathrm{x} S M N 2, \mathrm{n}=14 / 15 / 1$ ). Mean age (range) at dosing (days): 2xSMN2, 20.6 $(8.0-34.0) ; 3 x S M N 2,28.7(9.0-43.0)$. All patients are alive and none required ventilatory support as of last visit. Among $2 \mathrm{x} S M N 2$ patients, all have achieved CHOP INTEND scores $\geq 50$, which exceeds the maximal score observed in untreated patients; 8 sat (all within the WHO $1^{\text {st }}$ $99^{\text {th }}$ percentile; range: $5.7-11.8$ months); 4 stood and walked independently (range: $12.2-18.3$ months). Among $3 \times S M N 2$ patients, 4 stood independently (9.5-12.4 months). Remaining patients in both cohorts who have not achieved these milestones have not yet passed the WHO window. All patients evaluated at 6 or 12 months demonstrated intact swallowing. 30/30 patients experienced $\geq 1$ AE; 17/30 experienced treatment-related AEs. All serious AEs resolved and were considered unrelated to treatment.

Conclusions: Data show that presymptomatic SMA patients dosed with onasemnogene abeparvovec continued to meet primary endpoints, demonstrating a significant therapeutic benefit.
Adult-onset Krabbe's disease: clinical presentation and characterization of peripheral nerve involvement

M. Tagliapietra ${ }^{1}$, F. Crescenzo ${ }^{1}$, D. Polo $^{1}$, S. Ferrari ${ }^{2}$, T. Cavallaro ${ }^{2}$, G. Zanette $^{3}$ and G.M. Fabrizi ${ }^{1}$

${ }^{1}$ Department of Neuroscience, Biomedicine and Motor Sciences, University of Verona, Verona, Italy

${ }^{2}$ Neurology Division, Department of Neuroscience AOUI Verona, Verona, Italy

${ }^{3}$ Neurology Division, Pederzoli Hospital, Peschiera del Garda, Verona, Italy

We describe a case of adult-onset Krabbe's disease (globoid-cell leukodystrophy), a rare occurrence with possibly misleading clinical features of both central and peripheral involvement together with findings of hypomyelination and characteristic inclusions on nerve biopsy.

A 64-year-old gentleman presented with a 9-year history of progressive spastic-ataxic gait and urge incontinence. On examination, hypopallesthesia of the lower limbs with disto-proximal gradient, mild distal muscular atrophy, diffuse hyperreflexia except diminished ankle jerk reflex were also noted. Brain MRI revealed diffuse bilateral parieto-occipital white matter alterations and atrophy of the posterior body and splenium of the corpus callosum. Motor and visual evoked potentials testing showed conduction slowing of the central pathways. Cerebrospinal fluid analysis was unremarkable.

Upon further evaluation, concomitant involvement of the peripheral nerves was evident on nerve conduction studies as a sensory-motor polyneuropathy of the four limbs with diffuse slowing. Nerve ultrasound revealed diffuse, symmetrical hypertrophy of the peripheral nerves, that was more evident in their proximal portion, and of the cervical roots.

Biopsy of the right sural nerve showed marked features of a diffuse hypomyelinating disorder with lysosomal inclusions, in the absence of frank demyelinating changes.

In the suspicion of Krabbe's disease, molecular analysis of GALC gene were performed with detection of a pathogenic mutation at cDNA position $857 \mathrm{G}>\mathrm{A}$ (Gly286Asp), in compound heterozygosity with a deletion of exons 11-17.

Severe Charcot-Marie-Tooth disease type $1 \mathrm{~A}$ in a patient with PMP22 tetrasomy

F.Taioli ${ }^{1}$, GP. Zanette $^{2}$, M. Ferrarini ${ }^{1}$, S. Testi ${ }^{1}$, T. Cavallaro ${ }^{3}$, G.M. Fabrizi $^{1}$

${ }^{I}$ Section of Neurology, Department of Neuroscience, Biomedicine and Movement Sciences, University of Verona, Verona, Italy

${ }^{2}$ Neurology Unit, Pederzoli Hospital, Peschiera del Garda, Italy

${ }^{3}$ Section of Neurology B, Department of Neuroscience, University Hospital G.B. Rossi, AOUI Verona, Verona, Italy.

Charcot-Marie-Tooth disease type-1A (CMT1A) is caused by the trisomy of a $1.4 \mathrm{Mb}$ segment in chromosome $17 \mathrm{p} 12$ containing the peripheral myelin protein 22 gene (PMP22). Animal models linked CMT1A to PMP22 over-expression. However, bioptic samples from CMT1A patients failed to reveal clear increases of PMP22 mRNA/proteins ( $\mathrm{Li} \mathrm{J}$, Ann Clin Transl Neurol 2014).

A 18-year-old woman born from non-consanguineous asymptomatic (not examined) parents presented a peroneal atrophy syndrome characterized by onset in childhood with delayed motor milestones, severe impairment of gait and balance requiring ankle-foot orthoses and early involvement of manual dexterity. Examination revealed: severe scoliosis; talipes equinovarus with clawfoot; steppage, waddling, ataxic gait; severe wasting and weakness of distal four limbs and, to a lesser extent, of proximal lower 
limbs; glove-stocking multimodal sensory loss; bilateral facial weakness; areflexia. Nerve conduction velocities (NCV) were markedly and uniformly slowed (motor NCV in the upper limbs $9 \mathrm{~m} / \mathrm{s}$ ). Nerve highresolution ultrasound revealed uniformly and diffusely increase of cross-sectional area with values in the usual CMT1A range. Multiplex ligation-dependent probe amplification (MLPA) and quantitative realtime PCR targeting $\mathrm{c} 17 \mathrm{p} 12$ and PMP22 demonstrated a tetrasomy in the proband, a trisomy in the mother and a no copy number variations in the father. Proband and mother had the same MLPA pattern consistent with a rearrangement smaller than the common duplication, nonetheless including the full genomic sequences of PMP22. Results suggested the origin of the triplication during maternal meiosis.

Extreme phenotypes in the proband and asymptomatic mother highlight the gene dosage sensitivity of $P M P 22$.

\section{Short- and long-term efficacy of lenalidomide therapy in patients with POEMS syndrome}

F. Terenghi ${ }^{1}$, F. Gentile ${ }^{1}$, F. Gallia ${ }^{1}$, P.E. Doneddu ${ }^{1}$, R. Mazza ${ }^{2}$, M. Ruiz $^{1}$, A. Nozza ${ }^{3}$, E. Nobile-Orazio ${ }^{1}$

${ }^{1}$ Neuromuscular and Neuroimmunology Service, Department of Medical Biotechnology and Translational Medicine, Milan University, Humanitas Clinical and Research Hospital IRCCS, Milan, Italy

${ }^{2}$ Department of Medical Oncology and Haematology, Humanitas Cancer Centre, Humanitas Clinical and Research Hospital IRCCS, Milan, Italy

${ }^{3}$ Unit of Internal Medicine, Oncology, Hospital of Vigevano, Pavia, Italy

Autologous stem cell transplantation (ASCT) and radiotherapy are the main therapies of POEMS syndrome. Lenalidomide is also effective but its long-term efficacy remains unclear.

We treated with lenalidomide 21 patients with POEMS from 2009 to 2018 and 13 of them had a follow-up of at least 2 years. We defined efficacy as an improvement in at least two outcome measures (neurologic, clinical, VEGF, hematologic). Progression-free survival (PFS) and predictors of long-term remission were also analyzed.

Lenalidomide was the first-line therapy in 7 patients, while 14 were previously treated, mostly with ASCT (57\%). Patients received a median number of 23 cycles. Two patients suspended therapy for early adverse events while $17 / 21$ patients (81\%) improved after therapy. VEGF and clinical response had the highest rates $(86 \%$ and $81 \%)$, followed by neurological $(71 \%)$ and hematological response $(67 \%)$. Neurological improvement progressed for up to 2 years in $19 \%$ of the patients. Median follow-up was 3 years (range 1-10). Among the 13 patients with at least 2 years of follow-up, the PFS was $100 \%, 83 \%$ and $61 \%$ at 2-, 3- and 5years. Two patients maintained long-term remission for 7 years, and one was disease-free after 9 years. Complete hematological response $(p=$ $0,053)$ and achievement of at least 3 outcome measures $(p=0,07)$ showed a tendency as favorable prognostic factors.

Lenalidomide is a safe and effective therapy for POEMS syndrome. Prolonged treatment is feasible and may be associated with gradual, progressive improvement. Long-term remission is maintained in $61 \%$ of patients after 5 years.

\section{HyperCKemia and viral infections: a lesson from COVID-19}

\author{
C.Terracciano ${ }^{1}$, D. Zaino ${ }^{1}$, P. Immovilli ${ }^{1}$, E.Terlizzi ${ }^{1}$, D. Guidetti ${ }^{1}$ \\ ${ }^{1}$ Neurology Unit, “Guglielmo da Saliceto” Hospital, Piacenza, Italy
}

Viral infections are associated with a wide spectrum of muscular syndromes, ranging from myalgias to rhabdomyolysis. Muscle pain has been described as one of the most common neurological symptoms during
SARS-COV2 infection, and high levels of serum CK has been found in a large percentage of COVID-19 patients, even in those without muscular symptoms $^{1-2}$.

The mechanism underlying the development of myalgias and hyperCKemia during viral infections is not well understood. Different hypotheses have been formulated, such as direct viral invasion of myocytes, or a viral-mediated release of toxic cytokines ${ }^{3-4}$.

During pandemic we had the possibility to perform routine laboratory tests in 123 COVID-19 patients admitted to the Emergency Department of the hospital of Piacenza. Among these patients $46 \%$ showed increased CK values; using the Pearson correlation coefficient we found a strong direct correlation between CK levels and Neutrophil counts $\left(r=0.75, \mathrm{r}^{2}=\right.$ $0.56, \mathrm{p}$ value $<0.0001)$. Considering patients with $\mathrm{CK}$ levels indicative of rhabdomyolysis $(\mathrm{n}=8)$, this association was even stronger $(\mathrm{r}=0.98)$. Endogenous neutrophils have been described to have both supportive and detrimental effects on skeletal muscles in response to different stimuli (muscle ischemia, stretching or bacterial infection) $)^{5}$. IL-6 and other proinflammatory cytokines have been suggested to promote muscle damage through neutrophil activation ${ }^{6}$.

SARS-COV2 infection is associated with increased CK levels which appear to correlate to Neutrophil counts. Further studies are needed to verify whether this association is also present in other viral infections, and to understand the role of neutrophils in viral-related rhabdomyolysis.

1. Karadaş Ö, Öztürk B, Sonkaya AR. A prospective clinical study of detailed neurological manifestations in patients with COVID-19. Neurol Sci. 2020;41(8):1991-1995. doi:10.1007/s10072-020-04547-7.

2. Berger JR. COVID-19 and the nervous system. J Neurovirol. 2020;26(2):143-148. doi:10.1007/s13365-020-00840-5.

3. Naylor CD, Jevnikar AM, Witt NJ. Sporadic viral myositis in two adults. CMAJ. 1987;137(9):819-821.

4. Konrad RJ, Goodman DB, Davis WL. Tumor necrosis factor and coxsackie B4 rhabdomyolysis. Ann Intern Med. 1993;119(8):861. doi:10.7326/0003-4819-119-8-199310150-00024.

5. Dumont N, Bouchard P, Frenette J. Neutrophil-induced skeletal muscle damage: a calculated and controlled response following hindlimb unloading and reloading. Am J Physiol Regul Integr Comp Physiol. 2008;295(6):R1831-R1838. doi:10.1152/ajpregu.90318.2008.

6. Sugama K, Suzuki K, Yoshitani K, Shiraishi K, Kometani T. IL-17, neutrophil activation and muscle damage following endurance exercise. Exerc Immunol Rev. 2012;18:116-127.

\section{Peripheral nerve involvement in VCP-related multisystem proteinopathy.}

S. Testi ${ }^{1}$, M. Filosto ${ }^{2}$, A. Mazzeo ${ }^{3}$, M. Sabatelli ${ }^{4}, 5$, M. Luigetti ${ }^{5}$, E.

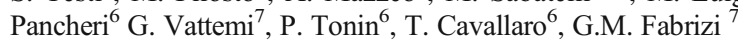

${ }^{1}$ Section of Neuropathology, Department of Neuroscience, University Hospital G.B. Rossi, AOUI Verona, Verona, Italy.

${ }^{2}$ Center for Neuromuscular Diseases, Unit of Neurology, ASST "Spedali Civili" and University of Brescia, Italy.

${ }^{3}$ Unit of Neurology and Neuromuscular Diseases, Department of Clinical and Experimental Medicine, University of Messina, Messina, Italy

${ }^{4}$ Centro Clinico NEMO - Roma, Rome, Italy.

${ }^{5}$ Neurology Unit, Fondazione Policlinico Universitario Agostino Gemelli IRCCS, Rome, Italy. Università Cattolica del Sacro Cuore, Rome, Italy.

${ }^{6}$ Section of Neurology B, Department of Neuroscience, University Hospital G.B. Rossi, AOUI Verona, Verona, Italy.

${ }^{7}$ Section of Neurology, Department of Neuroscience, Biomedicine and Movement Sciences, University of Verona, Verona, Italy

The Valosin Containing Protein (VCP), a ubiquitously expressed AAAATPase, plays multiple functions in organelle biogenesis and ubiquitindependent protein degradation. Mutations of $V C P$ cause multisystem 
proteinopathy (MSP) presenting variably with including inclusion body myopathy (IBM), Paget's disease of bone (PDB), Amyotrophic Lateral Sclerosis (ALS14), frontotemporal dementia (FTD) and, supposedly very rarely, Charcot-Marie-Tooth type 2 (CMT2Y).

Targeted next-generation sequencing identified three heterozygous $V C P$ variants with heterogeneous presentations. In three adult males (father and sons) a novel p.Ala160Ser substitution cosegregated with an axonal motor-sensory polyneuropathy (CMT2) manifesting in the first-second decade and exhibiting a slow progression with a moderately severe impairment in adulthood; the proband's muscle biopsy revealed neurogenic atrophy and rimmed vacuoles. In three adult males (father and sons) a previously reported $\mathrm{p}$.Arg93His substitution cosegregated variably with IBM, PDB, mild sensori>motor polyneuropathy and FTD. A c.1696$3 \mathrm{C}>\mathrm{T}$ variation previously described in a singleton case of ALS was found in a sporadic male affected with a lower-motor neuron (LMN) syndrome of the four limbs manifesting at age 49 years and requiring non-invasive positive ventilation 3 years after the onset; the LMN syndrome coexisted with mild clinical and neurophysiological signs of asymmetrical sensory neuropathy; a muscle biopsy revealed a neurogenic atrophy without rimmed vacuoles.

Sural nerve biopsies were consistent with a chronic axonal neuropathy in the p.Ala160Ser and p.Arg93His probands and with an active axonal neuropathy in the c.1696-3C $>\mathrm{T}$ proband.

The report highlights different patterns of peripheral nerve involvement in the heterogeneous spectrum of $V C P$-MSP.

\section{Movement disorders in children with a mitochondrial disease: a cross-sectional survey from the Nationwide Italian Collaborative Network of Mitochondrial Diseases}

C. Ticci ${ }^{1}$; D.Orsucci ${ }^{2}$; A. Ardissone ${ }^{3}$; E. Bertini ${ }^{4}$; C. Bruno ${ }^{5}$; V.Carelli ; D. Diodato ${ }^{4}$; A.M. Donati ${ }^{7}$; M. Filosto ${ }^{8}$; C.La Morgia ${ }^{6}$,C. Lamperti ${ }^{3}$; D. Martinelli ${ }^{4}$; C.Minetti ${ }^{5}$; M. Moggio ${ }^{9}$; T. Mongini ${ }^{10}$; V.Montano ${ }^{11}$; I. Moroni $^{3}$; O. Musumeci ${ }^{12}$; E. Pegoraro ${ }^{12}$; G.Primiano ${ }^{14}$; E. Procopio ${ }^{7}$; A. Rubegni $^{1}$; M. Sciacco ${ }^{9}$; S. Servidei ${ }^{14}$; G.Siciliano ${ }^{11}$; C.Simoncini ${ }^{11}$; P.Tonin ${ }^{15}$; A.Toscano ${ }^{12}$; M. Mancuso ${ }^{11}$; R. Battini ${ }^{11}$; F.M. Santorelli ${ }^{1}$

${ }^{I}$ IRCCS Fondazione Stella Maris, Pisa, Italy

${ }^{2}$ Unit of Neurology, San Luca Hospital, Lucca, Italy

${ }^{3}$ Fondazione IRCCS Istituto Neurologico Carlo Besta, Milan, Italy

${ }^{4}$ Bambino Gesù Hospital IRCCS, Rome, Italy

${ }^{5}$ Paediatric Neurology and Neuromuscular Disorders Unit, IRCCS

Istituto Giannina Gaslini, Genoa, Italy.

${ }^{6}$ IRCCS Institute of Neurological Sciences of Bologna, Bellaria Hospital, Bologna, Italy

${ }^{7}$ A. Meyer Children Hospital, Florence, Italy

${ }^{8}$ Center for Neuromuscular Diseases, Unit of Neurology, ASST "Spedali Civili" and University of Brescia

${ }^{9}$ Neuromuscular and Rare Diseases Unit, Fondazione IRCCS Ca' Granda Ospedale Maggiore Policlinico, Dino Ferrari Centre University of Milan, Milan, Italy

${ }^{10}$ Neuromuscular Unit, Department of Neuroscience "Rita Levi Montalcini", University of Torino, Torino, Italy

${ }^{11}$ Department of Clinical and Experimental Medicine, University of Pisa, Italy

12 Unit of Neurology and Neuromuscular Disorders, Department of Clinical and Experimental Medicine, University of Messina, Messina, Italy

${ }^{13}$ Neuromuscular Unit, Department of Neuroscience, University of Padova, Padua, Italy

${ }^{14}$ Fondazione Policlinico Universitario A. Gemelli IRCCS, Istituto di Neurologia Università Cattolica del Sacro Cuore, Rome, Italy

${ }^{15}$ Neurological Clinic, University of Verona, Verona, Italy
Movement disorders are increasingly being recognized as manifestation of mitochondrial diseases (MDs). However, the spectrum and characteristics of these conditions have not been studied in detail in the context of a well-defined cohort of patients.

We retrospectively explored a cohort of individuals with childhood-onset MDs querying the "Nationwide Italian Collaborative Network of Mitochondrial Diseases" (NICNMD) database (580 patients). Using a customized online questionnaire, we attempted to collect data from the subgroup of patients with movement disorders (197/580;34\%). Complete clinical, neuroradiological and molecular information was available only for 50/197 patients. Movement disorder was the presenting feature of MD in 22/50 (44\%) individuals, with a mean age at onset of 13 years. Ataxia was the most common manifestation at onset $(56 \%)$, followed by dystonia $(22 \%)$, tremor $(20 \%)$, chorea $(10 \%)$, hypokinetic disorders $(10 \%)$ and myoclonus (4\%). During the course of the disease, movement disorder worsened in most patients $(33 / 50 ; 66 \%)$, especially in those with extrapyramidal disorders at onset. The most frequent brain imaging abnormalities were basal ganglia involvement $(52 \%)$ and white-matter changes (44\%). Nineteen patients (38\%) harbored point mutations in the mitochondrial-DNA, $8(16 \%)$ had mtDNA rearrangements and $18(32 \%)$ carried mutations in nuclear-DNA-encoded genes, the latter associated with an earlier onset and a higher impairment in adaptive profiles. Thirteen individuals $(26 \%)$ received pharmacological treatment; clonazepam was the most commonly used drug and intrathecal baclofen the most effective. A better delineation of the movement disorders phenotypes in MDs starting in childhood may improve our diagnostic workup, management and treatment of affected patients.

\section{Muscle MRI of upper girdle in GNE myopathy}

E. Torchia ${ }^{1}$, M. Lucchini ${ }^{1}$, S. Bortolani ${ }^{2}$, M. Monforte ${ }^{2}$, M. Mirabella ${ }^{1,2}$, E. Ricci ${ }^{1,2}$, G. Tasca $^{2}$

${ }^{1}$ Università Cattolica del Sacro Cuore, Fondazione Policlinico Universitario A. Gemelli IRCCS, Roma, Italy

${ }^{2}$ Unità Operativa Complessa di Neurologia, Fondazione Policlinico Universitario A. Gemelli IRCSS, Rome, Italy

Background: GNE myopathy (GNEM) is a rare autosomal recessive adult-onset myopathy presenting with distal weakness and atrophy of lower limb (LL) muscles typically sparing the quadriceps. Muscle MRI pattern has been described in the LL with early involvement of biceps femoris short head and distal leg muscles and sparing of the vasti. Upper girdle involvement has never been investigated so far.

Objectives: to explore upper girdle involvement in GNEM patients with muscle MRI; to compare the severity of involvement with LL; to validate the LL pattern in unpublished patients.

Methods: we analyzed muscle MRI of the shoulder girdle in 6 GNEM patients. Fourteen muscles were examined on each side and degree of fatty replacement was scored using a 4-point scale. In four of these patients a LL scan was also available. Two additional unpublished patient were also studied by means of LL MRI only.

Results: the most common feature was fatty replacement of subscapularis muscle (5/6 patients). $3 / 6$ patients also had moderate-severe involvement of serratus anterior. All patients presented a more severe muscle involvement in LL. One patient had normal upper girdle scan despite extensive alterations in the LL. The MRI pattern in LL was confirmed also in the unpublished patients.

Conclusions: scapular girdle muscles are relatively spared, even in the advanced stage of GNEM, with the exception of subscapularis and serratus anterior muscles. We confirm the previously published pattern in the LL, with the additional observation of lumbar paraspinal involvement. 
Combined genetic causes of rhabdomyolysis in a family: can it be a double trouble?

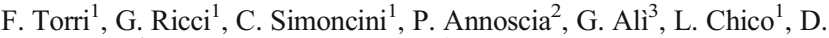
Cassandrini ${ }^{4}$, Di Muzio ${ }^{5}$, G. Siciliano ${ }^{1}$

${ }^{1}$ UO Neurologia, Dipartimento di medicina Clinica e Sperimentale, AOUP, Pisa, Italy

${ }^{2}$ UO Chirurgia della Mano, AOUP, Pisa, Italy

${ }^{3}$ UO Anatomia Patologica, AOUP, Pisa, Italy

${ }^{4}$ IRCCS Fondazione Stella Maris, Pisa, Italy

${ }^{5}$ Center for Neuromuscular Disease, CeSI, University "G. D'Annunzio", Chieti, Italy

Introduction: Rhabdomyolysis is a clinical condition characterized by muscular damage with acute increase of myolysis indicators, in association with myalgia, weakness and myoglobinuria. Exercise, infections, toxics, trauma, dysmetabolic or genetic conditions are the main causes. Among the genetic conditions, RYR1 and CPT2 genes are two common causes of rhabdomyolysis.

Case report: Our patient, a 45 years old male, reports a history of several episodes of rhabdomyolysis following physical exertion or fever. Similar symptoms are shared by his father and brother. Notably, his parents are consanguineous. Both our patient and his brother underwent muscle biopsy, showing minimal no specific myopathic changes. Genetic analysis of $C P T 2$ revealed that our patient is carrier of a homozygous mutation (c.338C $>\mathrm{T} / \mathrm{p} . \mathrm{S} 113 \mathrm{~L}$ ), while his brother is heterozygous for the same variant. Accordingly, the determination of CPT2 enzyme activity in brother's muscle was normal. Following that, through NGS analysis, a heterozygous variant in $R Y R 1$ gene of uncertain pathological meaning has also been found (c. $10018+8 \mathrm{G}>\mathrm{A}$, exon 66 ) in the proband, this prompting us to extend RYRl analysis in the other family members for further diagnostic definition.

Discussion: The use of NGS with the possibility to analyse several genes for studying muscle disorders is even more able to disclose unexpected complexity of muscle diseases also within the same families. Apparently identical phenotypes may be produced by mutations in different genes, in this remaining fundamental to assess the pathogenic weight of DNA variants with their timely clinical and molecular characterization.

Preliminary results of rank approach for improving quality of life in Charcot-Marie-Tooth Type 1A patients

S. Tozza ${ }^{1}$, D. Severi ${ }^{1}$, D. Bruzzese ${ }^{2}$, R. Iodice ${ }^{1}$, L. Ruggiero $^{1}$, R. Dubbioso $^{1}$, E. Spina ${ }^{1}$, A. Iovino ${ }^{1}$, F. Aruta $^{1}$, M. Bellofatto ${ }^{1}$, M. Nolano $^{1}$, L. Santoro ${ }^{1}$, F. Manganelli ${ }^{1}$.

${ }^{1}$ Department of Neuroscience, Reproductive and Odontostomatological Sciences, University of Naples "Federico II", Naples, Italy;

${ }^{2}$ Department of Public Health, University of Naples "Federico II", Naples, Italy.

Background: Charcot-Marie-Tooth type 1A (CMT1A) is due to duplication of PMP22 gene and it is the most common form of genetic neuropathy. Disability is related especially to motor symptoms with walking difficulties.

Aim: To understand, by using rank analysis, which are the most impacting symptoms on QoL in CMT1A patients and if symptoms change during the disease course.

Methods: We recruited 41 CMT1A patients and we asked patients to classify the following symptom categories from the greatest (1) to the lowest (5) impact on their QoL: "weakness of lower limbs" (WLL), "weakness of upper limbs" (WUL), "skeletal deformities" (SD), "sensory symptoms" (SS), "balance" (B).
Results: The symptom category with the highest impact on QoL was WLL, followed by B, SD, SS and lastly WUL. Dividing for median age (52 years), significant change $(p=0.043)$ of rank between the two group was found: the youngest CMT1A patients referred WLL, followed by SD, as symptom category with the greatest impact on QoL, while B represented the symptom category with the highest impact on QoL in the oldest CMT1A patients.

Conclusions: Overall, this study showed that weakness of lower limbs represents in CMT1A patients the most important symptom impacting on QoL. In particular, the youngest patients complained especially weakness of lower limbs and deformity, whereas in the oldest patients the QoL was influenced by balance. This rank approach could help clinician in attempting to improve the patient's QoL, suggesting reinforcement strength exercises of lower limbs and foot surgery in the youngest age and especially balance exercises in elderly.

\section{Clinical Features In ATTRv Patients}

S. Tozza ${ }^{1}$, D. Severi ${ }^{1}$, P. Origone ${ }^{2}$, A. Geroldi ${ }^{2}$, E. Bellone ${ }^{2}$, P. Mandich $^{2}$, M. Nolano ${ }^{1}$, L. Santoro ${ }^{1}$, F. Manganelli ${ }^{1}$.

${ }^{1}$ Department of Neuroscience, Reproductive and Odontostomatological Sciences, University of Naples "Federico II", Naples, Italy;

${ }^{2}$ Department of Neuroscience, Rehabilitation, Ophthalmology, Genetics and Maternal and Child Health (DINOGMI), University of Genoa, and IRCCS Policlinico San Martino, Genoa, Italy.

Background: Familial Amyloidosis Polyneuropathy (FAP) due to mutation in TTR gene is a rare disorder with a disabling and fatal course. It is clinically characterized by a severe and progressive sensory motor axonal neuropathy. Aim: To define the clinical features of patients with Amyloidosis TTR variant (ATTRv)-FAP.

Methods: We recruited 44 patients that were seen between 2011 and 2019 in our tertiary care neuromuscular center with sensory-motor idiopathic axonal polyneuropathy (IAP) and underwent TTR genetic analysis. Clinical features as progressive disease (referred to last 6-12 months), disability as assessed by FAP-stage, presence of motor, sensory and autonomic symptoms and bilateral carpal tunnel syndrome were compared with genetic results by using chi-square test.

Results: Out of the 44 patients with IAP, 14 patients (31.8\%) resulted positive for TTR mutation (57.2\% Val30Met; $42.8 \%$ Phe64Leu). The significant characteristics of ATTRv patients were a progressive disease $(p=0.009)$, severe disability as assessed by FAP-stage $(p=0.005)$, presence of motor symptoms $(p=0.016)$ and bilateral carpal tunnel syndrome $(p=0.001)$. No main features were sensory $(p=0.220)$ and autonomic symptoms $(p=0.133)$.

Conclusions: Our results suggested that one third of patients with IAP had ATTRv. The clinical profile of our ATTRv-FAP cohort was characterized by a progressive disease with predominant motor symptoms, bilateral carpal tunnel syndrome and severe disability. Conversely sensory and autonomic symptoms did not appear relevant to discern between IAP and ATTRv-FAP in our population.

\section{Childhood Chronic Inflammatory Demyelinating Polyradiculoneuropathy: A Case Series}

A. Tozzo ${ }^{1}$, A. Ardissone ${ }^{1}$, C. Ciano $^{2}$, FR. Danti ${ }^{1}$, E. Pagliano ${ }^{1}$, I Moroni ${ }^{1}$ ${ }^{1}$ Department of Pediatric Neurosciences,
${ }^{2}$ Neurophysiopatology Unit, Fondazione IRCCS, Istituto Neurologico
Carlo Besta, Milan

Chronic inflammatory demyelinating polyradiculoneuropathy (CIDP) is a treatable, immune mediated disorder of the peripheral 
nerves. CIDP is less common in children than adults, with reported incidence 0.48 per 100.00 ; knowledge of clinical characteristics, response to treatment and prognosis are still based on small pediatric case series.

We retrospectively evaluated data of 21 children, referred to our Institute in past years, with possible or confirmed CIDP according to the revised diagnostic criteria for childhood CIDP 2000. We focused on clinical, laboratory and electrophysiological features and response to treatment.

Eleven patients were females; the age of onset varied from 3 to 16 years. Six children presented with acute, Guillain-Barré-like onset, while the other 15 cases showed a slowly progressive disease with subsequent monophasic or relapsing-remitting course. The main clinical features were loss of autonomous gait and pain or changes in sensitivity in lower limbs; cranial nerve involvement was evident in few cases. Elevated cerebrospinal fluid protein was detected in most cases. All patients underwent electrophysiological studies that showed severe demyelinating abnormalities in 19 cases.

Spinal MRI examination showed gadolinium enhancement of the cauda equina and lumbar spinal nerve roots in $42 \%$ of patients.

Treatment included IVIG often associated with steroids; second line immunosuppressive therapies were necessary in a minority of cases. About a third of patients showed complete recovery, in the others we observed unstable active disease that need to continue treatment over time. In our series we noticed a correlation between the neurological severity at onset, the clinical course and response to therapies.

Tubular aggregate myopathy: clinical and molecular characterization in STIM1 and ORAII mutated patients.

S. Tripodi ${ }^{1}$, L. Bello ${ }^{1}$, G. Minervini ${ }^{2}$, C. Reggiani ${ }^{2}$, S. Vianello ${ }^{1}$, V. Bozzoni $^{1}$, L. Caumo ${ }^{1}$, P.Riguzzi ${ }^{1}$, A. Lupi ${ }^{1}$, C. Bertolin ${ }^{1}$, G. Sorarù ${ }^{1}$, E. Pegoraro ${ }^{1}$.

${ }^{1}$ Myology Institute, Department of Neuroscience, University of Padova ${ }^{2}$ Department of Biomedical Sciences, University of Padova

Introduction: STIMI and ORAII mutations associated tubular aggregate myopathy (TAM) is a rare progressive muscle disease, caused by dysfunction of Store Operated $\mathrm{Ca}^{2+}$ Channel.

Material and methods: A cohort of 20 patients with TA at muscle biopsies underwent STIMI and ORAII genetic screening. A large family segregating STIM1 mutation and two independent ORAIImutated patients were identified. Mutations were characterized by in silico modeling and by in vitro functional calcium studies in patients dissected single muscle fiber. Patients' muscle biopsies were characterized. Clinical features, disease progression and muscle MRI were longitudinally evaluated.

Results and discussion: We found seven related patients carrying the known p.Leu92Val STIM1 mutation, and two patients carrying the p.Leu188Pro and p.Met139Ile ORAII novel mutations. In silico modeling predicted for all mutations a dominant gain-of-function mechanism with increased influx of calcium, while in vitro studies showed adaptative changes and decreased isometric strength in STIM1 fibers. ORAI1 muscle biopsy compared to STIMI showed coarser and wider tubular aggregates. STIM1-TAM patients showed a moderately severe predominantly proximal muscle disease, while ORAI1-TAM patients presented a pseudometabolic picture; miosis was found in both subsets. STIM1-TAM muscle MRI confirmed a specific pattern with diffuse involvement of the posterior compartment of the thighs and legs with relative sparing of gracilis and tibialis anterior muscles. In ORAII-TAM a slight involvement of the gluteus maximum was observed.

Conclusion: We reported two novel ORAII pathogenic mutations and a three-generation family carrying a known STIM1 mutation to expand the clinical spectrum of this rare disease.
A case of progressive, stepwise, asymmetrical pure motor neuropathy: MMN versus motor CIDP.

\section{S. Tronci, U. Del Carro, M. Filippi, R. Fazio.}

Dept. of Neurology, Vita-Salute San Raffaele University and San Raffaele Scientific Institute, Milan, Italy.

Typically multifocal motor neuropathy (MMN) involves progressive, multifocal weakness, which typically begins and predominates in the upper limbs. MMN shares some features with the other form of chronic immune-mediated neuropathies, but remains a distinct entity given its clinical features, electrophysiological criteria and response to immunomodulatory treatment. We describe a case of a patient in which clinical diagnostic criteria for MMN are not supported by electrophysiological diagnostic criteria.

A 43 year old man was admitted in neurology department for a three year history of asymmetrical distal hyposthenia in the lower limbs, without sensory or painful symptoms. After significant worsening in the last 6 months with asymmetrical weakness in the upper limbs despite treatment with subcutaneous immunoglobulin, his disability progressed up to the use of the walking cane. The neurological examination showed severe hypotrophy and weakness with asymmetric distribution (MRC sum score: 44/60), and twelfth left nerve palsy. A electroneuromyography (ENG/EMG) study showed signs of demyelination associated with probable CBs, and widespread axonal damage. Sensory nerve conduction study and somatosensory evocated potentials (SSEP) were normal. As a supportive criteria were found un elevated IgM anti-ganglioside GM1 antibodies in serum. These data led to the diagnosis of possible MMN according to EFNS/PNS guidelines. The treatment administered was high dosage intravenous immunoglobulin (IVIg), followed by cyclophosphamide (CTX), with clinical improvement at two months.

According to the guidelines, we can classify this case as possible MMN due to supportive criteria. However, both clinical and neurophysiological peciuliarities seem to describe an atypical MMN case.

\section{Inflammation in Children with Neuromuscular Disorders and Sleep Disordered Breathing}

F. Trucco ${ }^{1,2}$, E. Carruthers ${ }^{2}$, J. C. Davies MD ${ }^{2,3}$, A. Simonds ${ }^{4}$, A. Bush $^{2,3}$, H.Tan ${ }^{2}$.

${ }^{1}$ Department of Neuroscience, Rehabilitation, Ophthalmology Genetics and Mother and Infant Science, University of Genova, Italy

${ }^{2}$ Department of Paediatric Respiratory Medicine, Royal Brompton Hospital, London

${ }^{3}$ National Heart and Lung Institute, Imperial College, London, UK

${ }^{4}$ Sleep \& Ventilation Unit, Royal Brompton and Harefield NHS Foundation Trust, London, UK;

Introduction: Children with obstructive sleep apnoea have been shown to have elevated inflammatory markers, thought to be due to the intermittent hypoxia. We hypothesised that children with sleep disordered breathing due to neuromuscular disorders also have evidence of elevated airway and systemic pro-inflammatory cytokines.

Methods: One-year prospective study of consecutive neuromuscular children (age5-18years) undergoing overnight full polysomnography and morning collection of serum and breath condensate, analysed for cytokines (Interleukin-10, Interleukin-6, Interleukin-1 $\beta$, Tumour Necrosis Factor $\alpha$, high-sensitivity C-Reactive Protein, Intercellular and Vascular Adhesion Molecules ICAM-1, VCAM-1). Cytokine levels were related to Oxygen desaturation index (ODI) of $4 \%$, and levels of overnight transcutaneous carbon dioxide ( $\mathrm{tcCO} \geq 6.7 \mathrm{kPa}>2 \%$ sleep).

Results: 23 patients were included, median age 12.6 years (IQR 8.714.6). An abnormal $\mathrm{ODI} \geq 3 / \mathrm{h}$, was associated with higher serum IL-6 $(\mathrm{p}=0.02)$. Children with abnormally elevated $\mathrm{CO} 2$ overnight, defined as tcCO2 $\geq 6.7 \mathrm{kPa}(50 \mathrm{mmHg})>2 \%$ sleep time, had higher ICAM-1 and 
VAM-1. Mean CO2 levels correlated with serum ICAM-1 ( $\mathrm{r}=0.570$, $\mathrm{p}=0.026)$ and VCAM-1 $(\mathrm{r}=0.76, \mathrm{p}=0.001)$. Correlation with EBC cytokines was not significant due to the small number of samples obtained. Discussion: SDB in neuromuscular children is associated with raised serum IL-6, VCAM-1, ICAM-1. This may predispose these children to future cardiovascular and other co-morbidities.

Long-term respiratory function in SMA type 2 and non-ambulant SMA type 3, longitudinal data from the international SMA consortium (iSMAc)

F.Trucco ${ }^{1,2}$, M. Scoto ${ }^{2}$, D. Ridout ${ }^{3}$, D. C. De Vivo ${ }^{4}$, B. Darras MD ${ }^{5}$, E. Bertini MD ${ }^{6}$, G. Coratti ${ }^{7}$, M. Main ${ }^{2}$, A. Mayhew ${ }^{8}$, J. Montes ${ }^{4,}{ }^{9}$, R. S. Finkel ${ }^{10}$, E. Mercuri ${ }^{7}$ and F. Muntoni MD ${ }^{2,11}$ on behalf of the international SMA consortium (iSMAc) ${ }^{12,13,14}$

${ }^{1}$ Department of Neuroscience, Rehabilitation, Ophthalmology Genetics and Mother and Infant Science, University of Genoa, IRCCS Istituto G. Gaslini, Genoa, Italy

${ }^{2}$ Dubowitz Neuromuscular Centre, UCL GOS Institute of Child Health, London

${ }^{3}$ Population, Policy and Practice Programme, UCL GOS Institute of Child Health, London

${ }^{4}$ Departments of Neurology, Columbia University Medical Center, New York, USA

${ }^{5}$ Department of Neurology, Boston Children's Hospital and Harvard Medical School, Boston, MA, USA

${ }^{6}$ Unit of Neuromuscular and Neurodegenerative Disorders, Post-Graduate Bambino Gesù Children's Research Hospital, IRCCS, Rome, Italy.

${ }^{7}$ Paediatric Neurology and Centro Clinico Nemo, Catholic University and Policlinico Gemelli, Fondazione Policlinico Universitario Agostino Gemelli IRCSS, Rome, Italy

${ }^{8}$ John Walton Muscular Dystrophy Research Centre, Newcastle University, UK

${ }^{9}$ Departments of Rehabilitation and Regenerative Medicine, Columbia University Medical Center, New York, USA

${ }^{10}$ Division of Neurology, Department of Pediatrics, Nemours Children's Hospital, Orlando, Florida, USA

${ }^{11}$ NIHR Great Ormond Street Hospital Biomedical Research Centre, Great Ormond Street Institute of Child Health, University College London, \& Great Ormond Street Hospital Trust, London, UK

12 SMA REACH UK Network

${ }^{13}$ Italian Telethon Network

${ }^{14}$ The US Pediatric Neuromuscular Research Network for SMA (PNCR)

\section{Collaborators:}

UK - Great Ormond Street Hospital; University College London; Birmingham Heartlands Hospital; Leeds Children Hospital; Evelina Children's Hospital, London; The Robert Jones and Agnes Hunt Orthopaedic Hospital, Oswestry; Sheffield Teaching Hospital

Italy - Centro Nemo Roma; Centro Nemo Milano; Centro Nemo Messina; Gaslini Children's Hospital, Genova; Bambino Gesu Hospital, Roma; C. Mondino Neurological Institute, Pavia; C. Besta Neurological Institute, Milano

US - Nemours Children's Hospital, Orlando, FL; Stanford Univ, Medical Centre, Palo Alto, CA; Boston Children's Hospital, Boston, MA; Columbia Univ Medical Centre, New York, NY; Children's Hospital of Philadelphia, Philadelphia, PA

Introduction: To describe the respiratory trajectories and their correlation with motor function in an international paediatric cohort of type 2 and non-ambulant type 3 spinal muscular atrophy (SMA) patients.

Methods: Nine-year retrospective observational study of patients recruited in the iSMAc natural history study. We collected anthropometrics, forced vital capacity (FVC) absolute, FVC\% predicted (FVC\%P.), Non-Invasive ventilation (NIV) requirement and co-morbidities affecting lung function. Hammersmith functional motor scale (HFMS) and Revised performance of upper limb (RULM) were correlated with respiratory function. We excluded patients in interventional clinical trials and on Nusinersen commercial therapy.

Results: There were 437 patients: 348 SMA type 2, 89 type 3. Mean age at first visit was $6.9( \pm 4.4)$ and $11.1( \pm 4)$ years. In SMA type $2 \mathrm{FVC} \% \mathrm{P}$. declined by $4.2 \%$ year from 5 to 13 years, followed by a slower decline (1.0\%/year). In SMA type $3 \mathrm{FVC} \% \mathrm{P}$. declined by $3.2 \%$ year until 13 years, also followed by a slower decline (1.4\%/year). 39\% SMA type 2 and 9\% SMA type 3 required NIV by the age of $6.5(1.8-16.6)$ and $15.1( \pm 1.8)$. Scoliosis was present in $84 \%$ SMA type 2 and $80 \%$ SMA type $3.54 \%$ and $46 \%$ respectively had a surgical procedure, which did not affect respiratory decline. $\mathrm{FVC} \% \mathrm{P}$ positively correlated with HFMS and RULM in both SMA subtypes.

Discussion: In SMA type 2 and 3 lung function declines differently preand post the age of 13 years. Lung and motor functions correlated in both type 2 and 3 . This data will help determining the long-term efficacy of new treatments for SMA.

\section{Efficacy of Rituximab in refractory Myastenia gravis}

F. Tuccillo, B.M. De Martino, M. Pezzella, M. Esposito, F. Habetswallner

U.O.C. Neurophysiology, A.O.R.N. Cardarelli, Naples, Italy

Myasthenia gravis is a disorder of the neuromuscular junction caused in the majority of the cases by antibodies against the acetylcoline receptor or muscle specific kinase. When patient fail to respond to thymectomy and at least 1 or 2 successive immunosuppressive drugs, with or without associated oral corticosteroids, they are usually considered to have treatment refractory disease (RM). The aim of this retrospective study is to evaluate the clinical benefit in our cohort of patients with refractory myasthenia gravis treated with rituximab. 23 patients $(8 \mathrm{~m}, 15 \mathrm{~F})$ were analyzed in the period from 2014 to 2020 . Outcome measures include the postintervention status(PIS) and the reduction of corticosteroid dosage or other immunosuppressant agents. Among our cohort 16 patients were positive for anti-AchR antibodies, 7 for Musk.

We performed our study at the neuropsysiological unit of Neurophysiology of A.O.R.N. "A. Cardarelli". Patients with refractory myastenia gravis received rituximab $1 \mathrm{~g}$ in two doses separeted by 15 days. In case of relapse patients underwent another infusion of $1 \mathrm{gr}$. In the post-treatment period (range 6-50 months), 14/16 AChR MG patients improved and 5 achieved the status of remission while the other 6 presented minimal manifestation (MM3). In contrast, 6/7 MuSK MG patients achieved a remission, 1 patient improved (problems related to other disease as parkinsonism). We observed a significant reduction in corticosteroid dosage and other immunosuppressant drugs but they could be withdrawn in only 2/16 AchR-patients while in the MuSKMG group, drugs were stopped in all but one case which is on low dose of prednisone (10 mg).

First and second line treatment in chronic inflammatory demyelinating polyradiculoneuropathy in children: 10 years experience of tertiary pediatric neurology centre.

F. Ursitti ${ }^{1}$, L. Papetti ${ }^{1}$, M.A.N. Ferilli ${ }^{1}$, R. Moavero ${ }^{1,2}$, G. Sforza ${ }^{2}$, F. Vigevano $^{1}$, M. Valeriani ${ }^{1,3}$

${ }^{1}$ Child Neurology Unit, Bambino Gesu' Children's Hospital, Rome, Italy ${ }^{2}$ Child Neurology and Psychiatry Unit, Tor Vergata University of Rome, Italy

${ }^{3}$ Center for Sensory-Motor Interaction, Aalborg University, Aalborg, Denmark Neurology Unit

Aim: Chronic inflammatory demyelinating polyradiculoneuropathy (CIDP) is a very rare condition in children. The therapy consists of 
the use of first-line treatment, as Immunoglobulin, Corticosteroids and Plasmapheresis and second-line treatment, as Azathioprine, Mycophenolate and Rituximab. To date, few clinical studies in the literature confirm the efficacy of the immunomodulatory treatment in developmental age.

The aim of the study was to observe the response to first and second line treatment in our patients.

Materials and Methods: we retrospectively analyzed the data of patients with CIDP referred to our Center from 2008 to 2018. Eighteen patients (ten males and eight females, mean age 9 years, range 3-18 years) with CIDP met the diagnostic criteria reported by Nevo et al in 2000 .

Results: all eighteen patients received first treatment with intravenous Immunoglobulin. Twelve responded to IV Immunoglobulin therapy, while six needed IV corticosteroids. Two out of these six patients underwent plasmapheresis, without efficacy. All six patients were administered second-line treatment with Azathioprine and/or Mycophenolate with poor efficacy. Finally, one patient received intravenous Rituximab therapy, with partial efficacy.

Conclusions: our data show that most our patients with CIDP responded to first-line therapy with IV Immunoglobulin and one third of patients needed to perform subsequent second-line treatment, with poor efficacy. To date, future multicenter studies are necessary to determine the effectiveness of first and second line treatments for CIDP in children.

Promoting Early DIagnoses in Neuromuscolar disease (PEDINE). A pilot study.

M. Vacchetti ${ }^{1}$, C. Brusa ${ }^{1,2}$, R. D’Alessandro ${ }^{2}$, M. Bobbio ${ }^{3}$, M. Spada ${ }^{4}$, R. Turra ${ }^{3}$, F. Ricci ${ }^{1,2,5}$, T. Mongini ${ }^{1}$, PEDINE working group.

${ }^{1}$ Department of Neurosciences "Rita Levi Montalcini", University of Turin, Italy.

${ }^{2}$ Department of Public Health and Paediatric Sciences, Section of Child and Adolescents Neuropsychiatry, University of Turin, Italy.

${ }^{3}$ Community Paediatrician, Turin, Italy.

${ }^{4}$ Department of Paediatrics, AOU Città della Salute e della Scienza, Regina Margherita Children Hospital, Turin, Italy.

${ }^{5}$ Division of Child Neurology and Psychiatric, AOU Città della Salute e della Scienza, Regina Margherita Children Hospital, Turin, Italy.

Over the last few years an increasing number of treatments for neuromuscular disorders have been developed, in some cases leading to the approval from Regulatory Authorities. As a common experience, there are strong evidences that the earlier the treatment the better the outcome, highlighting the importance of an early diagnosis.

PEDINE is a pilot project aiming to promote early diagnosis of neuromuscular diseases in children in the metropolitan area of Turin (Italy), through a structured collaboration of our Referral Centre with Community Paediatricians and local Neuropsychiatric Services.

In the first exploratory phase of three months, a total of 30 Paediatrician were provided with a dedicated email address to refer children with 'red flags' signs of suspected muscular impairment identified during the periodic visits scheduled as per National Health System policies for children. The Referral Centre organised 2 training sessions to share the protocol. A summary table with "red flags" illustrations was created to facilitate the assessments. During this study the Paediatricians performed 1871 scheduled visits. A total of 13 patients were referred to our Centre. No neuromuscular disorders were diagnosed, as expected from epidemiological data. One patient was diagnosed with a rare genetic disease and another one with a neurodevelopmental disorder. One patient is still undergoing diagnostic assessments.

This pilot project provided Community Paediatricians a standardised procedure to identify and promptly refer children with suspected signs of muscular involvement, and the dedicated email address proved to be an easy tool able to significantly reduce referral times.

\section{Muscle MRI as a novel outcome measure of hereditary transthyretin} amyloidosis: a cross-sectional cohort study

E. Vegezzi ${ }^{1,2}$, A. Cortese $^{2,3}$, N. Bergsland ${ }^{4}$, R. Mussinelli ${ }^{5}$, M. Paoletti ${ }^{1}$, F. Solazzo ${ }^{1}$, R. Currò ${ }^{1,2}$, A. Lozza ${ }^{5}$, X. Deligianni ${ }^{6}$, F. Santini ${ }^{6}$, S. Bastianello $^{1,2}$, G. Merlini ${ }^{5,2}$, G. Palladini ${ }^{5,2}$, L. Obici ${ }^{5 *}$ and A. Pichiecchio ${ }^{1,2 *}$

${ }^{1}$ IRCCS Mondino Foundation, Pavia, Italy;

${ }^{2}$ University of Pavia, Pavia, Italy;

${ }^{3}$ Department of Neuromuscular Disease, UCL Queen Square Institute of Neurology and The National Hospital for Neurology and Neurosurgery, London, UK;

${ }^{4}$ Buffalo Neuroimaging Analysis Center, State University of New York, USA;

${ }^{5}$ Amyloidosis Research and Treatment Center, Fondazione IRCCS Policlinico San Matteo, University of Pavia, Pavia, Italy;

${ }^{6}$ University Hospital Basel, Petersgraben 4, 4031, Basel, Switzerland *equal contribution

Background: The development of reliable outcome measures correlating with patient functional deficits and sensitive to early disease stages has proven challenging in hereditary transthyretin (hATTR) amyloidosis. Recently, magnetic resonance imaging (MRI) quantification of intramuscular fat showed high responsiveness in patients carrying Charcot-Marie-Tooth $1 \mathrm{~A}$.

Objective: To assess the role of quantitative muscle MRI as an outcome measure by cross-sectional correlation against functionally relevant clinical measure in hATTR amyloidosis.

Material and Methods: Twenty-nine patients with hATTR amyloidosis (22 males, median age 61, range 38-76) attending the Amyloidosis Research and Treatment Centre (Pavia) were enrolled. Most frequent mutations were Val30Met $(7 ; 24 \%)$ and Glu89Gln $(6$; $21 \%)$. Twenty-one/29 (72\%) patients were receiving treatment. Participants underwent Neurological Impairment Score (NIS) assessment and lower limb 3T-MRI of thigh and calf muscles. Muscle fat fraction (FF), indicating the degree of fat replacement of degenerated muscle fibers, was quantified with a 6-point Dixonbased method.

Results: Median NIS was 13 (range 0-170.5). Mean FF at thigh and calf level was $9 \pm 4 \%$ and $11 \pm 9 \%$, respectively, which is higher compared with mean FF of healthy controls of previous reports $(\sim 1.5 \%)$. We identified a significant correlation between mean FF and NIS at both thigh level and calf level $(\mathrm{p}=0.00)$. This positive association was independent from age, gender, mutation and treatment in a multivariate regression model.

Discussion and conclusion: This cross-sectional pilot study showed correlation between muscle MRI quantitative measures and clinical severity in ATTR amyloidosis. Responsiveness to change of quantitative MRI is currently being assessed in a prospective observational study.

Histopathological features in ambulant patients with Becker Muscular Dystrophy: preliminary data from the Givinostat trial cohort

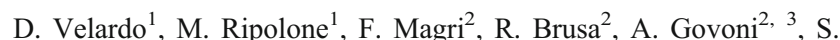
Cazzaniga $^{4}$, L. Peverelli ${ }^{5}$, P. Ciscato ${ }^{1}$, S. Zanotti ${ }^{1}$, M. Sciacco ${ }^{1}$, M. 


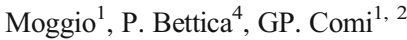

${ }^{1}$ Neuromuscular and Rare Disease Unit, Department of Neuroscience, IRCCS Foundation Ca' Granda Ospedale Maggiore Policlinico, University of Milan, Milan, Italy.

${ }^{2}$ Neurology Unit, Neuroscience Section, Department of Pathophysiology and Transplantation (DEPT), Dino Ferrari Centre, University of Milan, IRCCS Foundation Ca' Granda Ospedale Maggiore Policlinico, Milan, Italy.

${ }^{3}$ Neurological Clinics, Department of Clinical and Experimental Medicine, University of Pisa, Pisa, Italy.

${ }^{4}$ Italfarmaco S.p.A., Milan, Italy.

5 ASST Lodi, Ospedale Maggiore di Lodi distretti e Presidi Lodigiani,U.O. Neurologia, Italy

In our Center, we are currently performing a randomised, double-blind, placebo-controlled study to evaluate the effects on muscles, the safety and tolerability, and the efficacy of givinostat in patients with Becker Muscular Dystrophy (BMD). Ambulant adult male patients with a molecular diagnosis of BMD and a screening 6 minutes walking test between 200 and 450 meters, were recruited. All patients underwent biceps muscle biopsy before the randomization visit; a second biopsy will be performed at the end of the study.

The enrolment phase of the study has been recently closed and we have collected histological specimens from 48 patients.

Histological, histochemical, immunohistochemical stainings and morphometric analysis were performed in order to evaluate muscle fiber area fraction, percentage of fibrosis, percentage of adipose tissue, mean fiber cross-sectional area, regenerating fibers and dystrophin quantification. Although some analysis are still underway, we can already present some of them. In particular, the histopathological features showed a significant fibro-adipose replacement, probably due to the chronicity of the disease, in $2 / 3$ of patients.

In addition, the first 22 biopsies showed marked fiber size modifications,

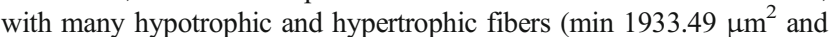
$\max 9446.34 \mu \mathrm{m}^{2}$ ) accounting for a very significant variability (S.D: $1904.86 \mu \mathrm{m}^{2}$ ).

At present, this work has collected one of the largest amounts of muscle biopsies, taken many years after the onset of the disease, in ambulant BMD patients. These data provide relevant information about the histopathological progression of the disease, that could be useful also for future pharmacological trials.

Severe inflammatory myopathy in a pulmonary carcinoma patient treated with Pembrolizumab: an alert for myologists

D. Velardo ${ }^{1}$, L. Peverelli ${ }^{2}$, A. De Rosa ${ }^{1}$, E. Domina ${ }^{2}$, P. Ciscato ${ }^{2}$, G. $\mathrm{Sita}^{3}$, M. Sciacco ${ }^{1}$, M. Moggio ${ }^{1}$, G.P. Comi ${ }^{1,4}$

${ }^{1}$ Neuromuscular and Rare Disease Unit, Department of Neuroscience, Foundation IRCCS Ca' Granda Ospedale Maggiore Policlinico, Milano, Italy.

${ }^{2}$ ASST Lodi, Ospedale Maggiore di Lodi distretti e Presidi Lodigiani,U.O. Neurologia, Lodi, Italy;

${ }^{3}$ ASST Lodi, Ospedale Maggiore di Lodi distretti e Presidi Lodigiani,U.O. Oncologia, Lodi, Italy;

${ }^{4}$ Dino Ferrari Centre, Neuroscience Section, Department of Pathophysiology and Transplantation (DEPT), University of Milan, Milan, Italy

Immune-correlated adverse events (icAEs) during the administration of immune-checkpoint inhibitors (ICBs) become more evident with the spreading of these therapies. We report a paradigmatic case characterised by severe systemic inflammatory myopathy and deep cardiac involvement abruptly precipitated in an untoward ending to remind the importance of early recognition of this phenomenon.

\section{Case Report}

The patient was a 60 years old man suffering from pulmonary squamous cell carcinoma with pleural-pericardial involvement. He received one single dose of pembrolizumab $200 \mathrm{mg}$ and after 7 days he started complaining of neck pain and walking difficulty.

He was admitted in emergency care and blood test examination revealed high increase of Creatine kinase (CK) level (20.000 U/1); the clinical examination showed head drop and proximal weakness.

EMG showed diffuse denervation along with myopathic signs suggestive of muscle inflammation. After one day he started to complain of diplopia and mild swallowing disturbances. He underwent to a muscle biopsy in the left biceps muscle that showed a severe, acute inflammatory myopathy.

Two days after starting steroid therapy, he presented acute heart failure and, despite of external Pace Maker implantation and vaso-active support, he passed away one day after.

In the days following the administration of ICBs, we recommend both a close clinical and blood CK monitoring in these patients. It would thus be possible to find out the first mild signs of myopathy without waiting for a clear symptomatology to bring the patient to the physicians.

\section{Molecular and clinical characterization in asymptomatic and symp-} tomatic DMD carriers.

V. Zangaro ${ }^{1}$, P. Riguzzi ${ }^{1}$, B. Merlo ${ }^{1}$, S. Vianello ${ }^{1}$, R. Bariani ${ }^{2}$, L. Lamagna $^{1}$, B. Bauce ${ }^{2}$, G. Sorarù ${ }^{1}$, L. Bello ${ }^{1}$, E. Pegoraro ${ }^{1}$

${ }^{1}$ Department of Neurosciences DNS, University of Padova, Padova Italy, ${ }^{2}$ Department of Cardiac, Thoracic and Vascular Sciences, University of Padova, Padova, Italy.

Objectives: To determine the molecular and clinical characteristics of a cohort of asymptomatic and symptomatic Duchenne muscular dystrophy (DMD) carriers.

Materials and methods: Seventy two female DMD carriers were enrolled (average age 46.3+- 14.0 years): 50 were asymptomatic and 22 showed muscle weakness.

Disease endpoints were collected (age at loss of ambulation, age at loss of ability to raise from the floor, age at cardiomyopathy onset). Muscle strength was assessed by MRC scale in proximal, distal and axial muscles. Transthoracic echocardiogram was done to assess heart function. Muscle biopsies, when available, were used to assess dystrophin amount by Western Blot analysis. Blood samples were collected to evaluate $\mathrm{CK}$ levels and for DNA analyses.

Pattern of X inactivation was determined by methylation sensitive restriction enzyme digestion. The polymorphic CAG repeat in the first exon of the androgen receptor was used to identify the two X chromosome alleles. Genotyping at selected gene modifiers (SPP1, LTBP4, CD40, ACTN3 haplotypes) was done.

Results: The clinical criteria for a manifesting carrier diagnosis were consistently elevated serum CK (above 1,000 IU/1) and myopathic histopathology. $D M D$ gene mutation in our cohort included 43 multiple exons deletion, 20 nonsense, 7 multiple exons duplication, and 2 complex $D M D$ gene rearrangement.

Among the 22 symptomatic carriers muscle weakness was moderate/ severe in 18/22 and 3 of these lost ambulation at a mean age of 54 years. In 4 patients no muscle weakness was detected but myalgias were reported.

We found a good correlation between extent of dystrophin deficiency, skewed X-inactivation pattern, serum $\mathrm{CK}$, and clinical phenotype in the symptomatic carriers. These correlations did not hold up in the asymptomatic carriers.

Seven of the 22 symptomatic carriers showed a dilated cardiomyopathy and two died for heart failure at age 65 and 71 years.

LTBPA genotyping showed a better cardiac profile in the IAMM haplotype DMD carriers. 
Discussion and conclusions: Accurate genotype/phenotype correlations in manifesting carriers of Duchenne dystrophy have been limited by the ability to accurately quantitate the degree of X inactivation and dystrophin content of muscle. Cardiac evaluation is foremost important in DMD symptomatic and asymptomatic carriers in a proactive therapy assessement.

Genotyping at modifier loci may help to define an at risk haplotype in DMD carriers.

\section{Subcutaneous immunoglobulins in Myasthenia Gravis and anti- HMGCR myositis}

\author{
A. Zuppa ${ }^{1,2}$, C. Demichelis ${ }^{1,2}$, S.G. Grisanti ${ }^{1,2}$, C. Cabona ${ }^{1,2}$, A. \\ Schenone $^{1,2}$, L. Benedetti ${ }^{1,2}$, M. Grandis ${ }^{1,2}$ \\ ${ }^{1}$ Department of Neuroscience, Rehabilitation, Ophtalmology, Genetics, \\ Maternal and Child Health (DINOGMI), Genova, Italy \\ ${ }^{2}$ IRCCS Policlinico San Martino Genova, Italy
}

Subcutaneous Immunoglobulins (SCIg) are an alternative to Intravenous immunoglobulins (IVIg) in inflammatory neuromuscular disorders with advantages of home administration, stable Ig serum level and reduced Ig Adverse Events (AEs). To date, few studies describe SCIg efficacy in myasthenia gravis (MG) and fewer data are available for anti-hydroxy-3methylglutaryl-coenzyme A reductase (anti-HMGCR) myositis. The aim is to describe clinical records of seven patients with generalized MG and two with anti-HMGCR myositis treated with $\mathrm{SCIg}$.

All MG patients were treated with pyridostigmine associated to prednisone or azathioprine. Seen the difficulties in maintaining long-term immunosuppressive therapy and attempting to achieve better clinical stability, all patients received IVIg treatment and subsequently switched to SCIg. The dose was converted varying from $95 \%$ a $150 \%$ of the previous IVIg monthly dose. Four patients are clinically stable during a median of 10 months. Two patients discontinued SCIg due to malabsorption and major surgery after 8 months and one year respectively. Finally the last one has a very long follow-up (4 years) during which she experienced two relapses both treated with a single IVIg cycle.

The two anti-HMGCR myositis patients were shifted to SCIg and methotrexate after the resolution of acute phase treated with conventional therapy and after 6 months they maintain clinical and CK normalization. In conclusion, we strengthen the usefulness of SCIg in patients with $\mathrm{MG}$ and anti-HMGCR myositis, especially in case of IVIg or immunosuppressive AEs. Clinical benefit can be maintained for more than one year, but possibly requiring dose implementation or additional IVIg cycle.

Publisher's note Springer Nature remains neutral with regard to jurisdictional claims in published maps and institutional affiliations. 